\title{
Gut liver axis in liver cirrhosis
}

Citation for published version (APA):

Pijls, K. E. (2016). Gut liver axis in liver cirrhosis. [Doctoral Thesis, Maastricht University]. Maastricht University. https://doi.org/10.26481/dis.20161209kp

Document status and date:

Published: 01/01/2016

DOI:

10.26481/dis.20161209kp

Document Version:

Publisher's PDF, also known as Version of record

\section{Please check the document version of this publication:}

- A submitted manuscript is the version of the article upon submission and before peer-review. There can be important differences between the submitted version and the official published version of record.

People interested in the research are advised to contact the author for the final version of the publication, or visit the DOI to the publisher's website.

- The final author version and the galley proof are versions of the publication after peer review.

- The final published version features the final layout of the paper including the volume, issue and page numbers.

Link to publication

\footnotetext{
General rights rights.

- You may freely distribute the URL identifying the publication in the public portal. please follow below link for the End User Agreement:

www.umlib.nl/taverne-license

Take down policy

If you believe that this document breaches copyright please contact us at:

repository@maastrichtuniversity.nl

providing details and we will investigate your claim.
}

Copyright and moral rights for the publications made accessible in the public portal are retained by the authors and/or other copyright owners and it is a condition of accessing publications that users recognise and abide by the legal requirements associated with these

- Users may download and print one copy of any publication from the public portal for the purpose of private study or research.

- You may not further distribute the material or use it for any profit-making activity or commercial gain

If the publication is distributed under the terms of Article $25 \mathrm{fa}$ of the Dutch Copyright Act, indicated by the "Taverne" license above, 
Gut liver axis in liver cirrhosis 
CC Copyright Kirsten Pijls, Maastricht 2016

ISBN: 978-94-028-0415-7

Layout: Tiny Wouters

Printing: Ipskamp Drukkers, Enschede

Printing of this thesis was financially supported by Kupffer Cell Foundation. 


\section{Gut liver axis in liver cirrhosis}

\section{PROEFSCHRIFT}

ter verkrijging van de graad van doctor aan de Universiteit Maastricht,

op gezag van de Rector Magnificus, Prof. dr. Rianne M. Letschert, volgens het besluit van het College van Decanen, in het openbaar te verdedigen op

vrijdag 9 december 2016 om 14:00 uur

door

Kirsten Evelyne Pijls 


\title{
Promotor
}

Prof. dr. A.A.M. Masclee

\section{Co-promoteres}

\author{
Dr. G.H. Koek \\ Dr. D.M.A.E. Jonkers
}

\section{Beoordelingscommissie}

Prof. dr. O. Bekers (voorzitter)

Prof. dr. C.H.C. Dejong

Prof. dr. H. Grabsch

Prof. dr. B. van Hoek, Leids Universitair Medisch Centrum

Prof. dr. S. van der Merwe, Universitair Ziekenhuis Leuven, België 


\section{Contents}

$\begin{array}{lll}\text { Chapter } 1 \quad \text { General introduction } & 7\end{array}$

Chapter 2 Intestinal epithelial barrier function in liver cirrhosis: an extensive 21 review of the literature

Chapter 3 Large intestinal permeability is increased in patients with compensated liver cirrhosis

Chapter $4 \quad$ Is intestinal oxidative stress involved in patients with compensated liver cirrhosis?

Chapter 5 Altered fecal and duodenal microbial profiles are associated with disease severity in liver cirrhosis patients

Chapter 6 The role of the intestine in animal models with diet-induced hepatic steatosis and steatohepatitis

Chapter 7 Critical appraisal of ${ }^{13} \mathrm{C}$ breath tests for microsomal liver function: aminopyrine revisited

Chapter 8 A profile of volatile organic compounds in exhaled air as a potential non-invasive biomarker for liver cirrhosis

Chapter 9 General discussion

Summary

Samenvatting

Valorisation

Dankwoord

Curriculum vitae

List of publications 

Chapter 1

General introduction 


\section{Liver cirrhosis}

Cirrhosis is the end stage of a variety of chronic liver diseases predominantly related to alcohol consumption, viral infections and metabolic disorders. It is characterized by distortion of normal liver architecture encompassing diffuse parenchymal fibrosis, nodules and vascular remodeling. ${ }^{1}$ According to the National Center for Health Statistics, cirrhosis and chronic liver diseases are the $12^{\text {th }}$ leading cause of death in the United States. ${ }^{2}$ In Europe, $1.8 \%$ of all deaths are caused by cirrhosis, accounting for approximately 170.000 deaths per year. ${ }^{3}$ Liver transplantation remains the only definite treatment for eligible cirrhotic patients and improves survival. ${ }^{4,5}$

The natural history of cirrhosis is usually characterized by a relatively long asymptomatic stage of compensated disease followed by a symptomatic stage of decompensated disease. Decompensated cirrhosis is defined by the development of clinically evident complications of portal hypertension or liver insufficiency: i.e. ascites, variceal hemorrhage, hepatic encephalopathy and/or jaundice. ${ }^{6}$ The development of complications, such as spontaneous bacterial peritonitis (SBP), hepatorenal syndrome, hepatopulmonary syndrome, and hepatocellular carcinoma (HCC) may accelerate further progression of cirrhosis. ${ }^{7}$ The transition from compensated to decompensated cirrhosis is associated with a marked reduction in life expectancy, which is mainly secondary to the complications. The reported median survival time for patients with compensated and decompensated cirrhosis is around 12 and 2 years, respectively. ${ }^{7}$ Ascites is usually the first and most frequent complication, associated with a one-year mortality rate of $20 \%{ }^{7}$ Another complication with a high mortality is SBP, which develops in up to $3.5 \%$ of cirrhotic outpatients and in around $12 \%$ of cirrhotic inpatients with ascites. ${ }^{8-10}$

The prognosis of cirrhosis in clinical practice is generally assessed by the Child-Pugh classification and the Model for End Stage Liver Disease (MELD) score by predicting survival and the need for transplantation, respectively. ${ }^{11-13}$ Furthermore, several factors have been found to be associated with clinical decompensation, such as the etiology, ${ }^{14}$ presence of obesity, ${ }^{15}$ serum albumin levels and the degree of portal hypertension. ${ }^{16}$

\section{Causes of cirrhosis}

The most common causes of cirrhosis are alcoholic liver disease (ALD), chronic infections by hepatitis $B$ and $C$ viruses (HBV and HCV) and non-alcoholic fatty liver disease (NAFLD) related to obesity and/or other components of the metabolic syndrome. Alcohol is the strongest risk factor for cirrhosis and in 2011, almost $50 \%$ of all deaths from cirrhosis were related to ALD. ${ }^{2}$ Chronic infections by HBV and HCV are also important determinants of cirrhosis. Cirrhosis is found in $20-30 \%$ of patients with chronic HBV infection ${ }^{3}$ and it has been estimated that approximately $16 \%$ of patients with chronic HCV infection develop cirrhosis within 20 years. ${ }^{17}$ Furthermore, NAFLD is 
the most common form of chronic liver diseases with a prevalence rate of $10-35 \%$ in the general population. ${ }^{18,19}$ It encompasses a spectrum that ranges from simple hepatic steatosis to steatohepatitis, i.e. non-alcoholic steatohepatitis (NASH), which in turn can progress in about $20 \%$ into cirrhosis, liver failure and eventually HCC. ${ }^{20}$ Because of the increasing prevalence of obesity, the prevalence of NASH-related cirrhosis is expected to rise even further in the future.

\section{The role of the intestine}

Recent studies indicate that apart from these etiological factors causing liver damage, the intestine is involved in the pathophysiology of chronic liver diseases and cirrhosis. Alterations in the intestine, such as bacterial overgrowth and dysfunction of the epithelial barrier have been found in patients with various chronic liver diseases, such as ALD $^{21-23}$ and NAFLD ${ }^{24,25}$ as well as in patients with cirrhosis. ${ }^{26-28}$ Moreover, the presence of intestinal alterations in cirrhosis is also suggested to contribute to the risk of developing cirrhosis-related complications.

In the subsequent paragraphs, the current knowledge on intestinal epithelial barrier function, oxidative stress and microbiota in patients with cirrhosis will be briefly reviewed.

\section{Intestinal epithelial barrier function}

The intestinal barrier can mainly be subdivided into an epithelial (i.e. the mucus layer and epithelial cells) and an immunological barrier (i.e. the epithelial secretions and immune cells). The epithelial intestinal barrier is essential to prevent the entry of potentially harmful luminal substances, such as bacteria and their products, into the portal and systemic circulation by means of paracellular and transcellular pathways. ${ }^{29}$ The paracellular pathway is mainly regulated by the apical intercellular junctional complex, which consists of tight junctions (TJs) and subjacent adherens junctions (AJs). ${ }^{30}$ The TJs are composed of several transmembrane proteins (e.g. occludin, members of the claudin family and junction adhesion molecules) that are linked with the perijunctional actomyosin ring through cytoplasmic plaque proteins (e.g. zonula occludens proteins). Myosin light chain kinase (MLCK) is an important intracellular mediator of paracellular permeability by inducing contraction of the perijunctional actomyosin ring through myosin II regulatory light chain (MLC) phosphorylation. ${ }^{31}$ Dysfunction of the intestinal epithelial barrier, facilitating enhanced bacterial translocation is increasingly recognized as a contributing factor in the pathophysiology of cirrhosis and its complications.

Enhanced translocation of bacteria and bacterial products, such as endotoxin, through the intestinal barrier into the portal circulation can activate Kupffer cells, subsequently leading to production of inflammatory mediators, which can induce liver injury. ${ }^{32}$ 
Studies have shown an increased small and whole intestinal permeability in mixed groups of compensated and decompensated cirrhotic patients. ${ }^{28,33-35}$ Changes seem to be most pronounced in patients with decompensated cirrhosis, i.e. those with complications. ${ }^{36,37}$ It is not clear whether the intestinal permeability is also increased in those with compensated cirrhosis and thereby might be a risk factor for the development of complications. Only two studies have assessed the expression of TJ proteins in the small intestine of patients with compensated and decompensated cirrhosis, but findings were not consistent. ${ }^{38,39}$ Data on the epithelial barrier function of the large intestine is scarce. One study, using a single marker, found an increased permeability of the large intestine in patients with cirrhosis. ${ }^{40}$

Various factors and mechanisms may affect the intestinal epithelial barrier function in the development of cirrhosis, such as alcohol consumption and high fat intake (obesity), both directly and indirectly by altering the intestinal microbiota or inducing inflammation/oxidative stress. ${ }^{41,42}$ Cirrhosis itself is associated with portal hypertension as well as alterations in the intestinal microbiota composition, ${ }^{43}$ systemic inflammation and oxidative stress, ${ }^{44-47}$ each of which can affect the barrier function and may contribute to the development of complications.

\section{Intestinal oxidative stress}

Oxidative stress is characterized by a disturbance in the oxidant and antioxidant balance in favor of the former. In patients with cirrhosis, oxidative stress in the intestine may result from alcohol consumption, ${ }^{48,49}$ alterations in intestinal microbiota, ${ }^{50,51}$ intestinal inflammation ${ }^{39,52}$ and a disturbed microcirculation of the intestinal mucosa secondary to portal hypertension. ${ }^{53-55}$ In addition, systemic inflammation and oxidative stress as well as oxidative stress inducers produced in the liver, may be transferred to the intestine via blood and bile. ${ }^{56,57}$ These factors together with a decreased antioxidant status in cirrhotic patients ${ }^{45}$ may promote intestinal oxidative stress. The presence of oxidative stress in the intestine may lead to direct cell damage and TJ and/or AJ disruption, subsequently resulting in barrier dysfunction. ${ }^{56,58,59}$

Few studies in rats with carbon tetrachloride $\left(\mathrm{CCl}_{4}\right)$-induced cirrhosis have shown evidence for oxidative stress in both the small and large intestine, by increased xanthine oxidase (XO) activity, increased malondialdehyde (MDA) levels, and alterations in antioxidant status. ${ }^{59-62}$ In cirrhotic rats with ascites, increased ileal and caecal MDA levels were accompanied by increased bacterial translocation, ${ }^{60,61}$ which were both attenuated after treatment with antioxidants. ${ }^{60}$ Only one study has been performed in patients with cirrhosis. Lipid peroxidation in biopsies of the small intestine was found to be higher in patients with decompensated cirrhosis when compared with those with compensated cirrhosis and healthy controls. ${ }^{63}$ To our knowledge, studies investigating intestinal oxidative stress in the large intestine in patients with liver cirrhosis are not available. 


\section{Intestinal microbiota}

The intestinal microbiota is a complex ecosystem colonizing the entire length of the intestine. At the phylum level, the microbiota is dominated by two phyla, i.e. Firmicutes and Bacteroidetes, whereas at lower taxonomic levels the microbiota consists of more than 1000 different species. ${ }^{64,65}$ The intestinal microbiota has several important functions for the host, including e.g. colonization resistance, its metabolic activity and influencing epithelial cell proliferation and differentiation, and the immune system. Increasing evidence indicates that the intestinal microbiota is involved in a variety of not only intestinal, but also systemic diseases, including inflammatory bowel disease, ${ }^{66,67}$ obesity $^{68,69}$ and diabetes. ${ }^{70,71}$

The intestinal microbiota is also found to be altered in patients with chronic liver diseases such as NAFLD ${ }^{72}$ and ALD. ${ }^{73}$ In cirrhosis, microbial perturbations are suggested to be an important factor that can enhance bacterial translocation, and thereby play a role in the progression of cirrhosis and its complications.

Overgrowth of microbiota in the small intestine has frequently been reported in cirrhotic patients by bacterial culture from jejunal aspirates. ${ }^{74-76}$ However, Steed et al. ${ }^{77}$ could not find bacterial overgrowth, except for enterococci, in mucosal biopsies of the small intestine by use of real-time qPCR. The presence of small bacterial overgrowth in cirrhosis can in part be attributed to an impaired motility and prolonged transit time that have been observed in some patients with cirrhosis. ${ }^{78}$

In recent years, several studies characterized the structure and function of the fecal microbiota in patients with compensated and/or decompensated cirrhosis in comparison with healthy controls by using sequencing approaches that require timeconsuming and expert bioinformatics support. An increase of Proteobacteria and Fusobacteria, and a corresponding decrease of Bacteroidetes in mixed groups of patients with compensated and decompensated cirrhosis versus healthy controls have been reported in some studies. ${ }^{50,79,80}$ The alterations in the microbiota composition seem to be more pronounced in patients with advanced cirrhosis, especially in those with complications, such as hepatic encephalopathy. ${ }^{51,81}$ Most of the abovementioned studies have focused primarily on the fecal microbiota in cirrhotic patients, whereas data on mucosa-associated microbiota are limited.

\section{Non-invasive diagnostics}

The presence of cirrhosis in clinical practice is often suggested by a combination of imaging an irregular and nodular liver with an impaired liver synthetic function, but histological evaluation of a liver biopsy is still the current standard for a definite diagnosis. ${ }^{82}$ Early diagnosis is essential to prevent progression towards decompensated cirrhosis, for example, by endoscopic screening of and prophylactic treatment for gastrointestinal varices ${ }^{83}$ and thereby improve the high morbidity and mortality. 
However, a liver biopsy is an invasive procedure associated with significant complications, $^{84,85}$ sampling error and inter- and intra-observer variability, ${ }^{86-88}$ and therefore not suitable for follow-up. For these reasons, non-invasive alternatives for differentiating patients with chronic liver diseases from those with compensated cirrhosis, i.e. for staging fibrosis, have been developed. They include serum biomarker panels and radiological tests, such as transient elastography, or combinations of these tests. At first, they were mostly developed in patients with HCV infection. Serum biomarker panels use parameters available from routine laboratory tests, such as the aspartate aminotransferase (AST) to platelet ratio (APRI) and the FIB-4 index, which can both accurately predict cirrhosis in HCV infected patients with reported area under the receiver operating characteristic (ROC) curve values of 0.83 and 0.91 , respectively. ${ }^{89,90}$ However, they estimate disease severity and do not reflect hepatic metabolic function or dynamic changes. ${ }^{91}$ Several breath tests using probe drugs as substrates have been applied to assess the functional metabolic capacity of the liver. In addition, specific combinations of volatile organic compounds (VOCs) in exhaled air were found to discriminate between cirrhotic patients and healthy controls, ${ }^{92-95}$ but the accuracy of exhaled VOCs in predicting the presence of compensated cirrhosis within a heterogeneous group of chronic liver disease patients has not yet been investigated. 


\section{Aims and outline of the thesis}

Based on the literature, there seems to be an interaction between the intestine and the liver in the pathophysiology of chronic liver diseases, cirrhosis and cirrhosis-related complications. We hypothesized that an altered intestinal barrier and microbiota composition is associated with compensated liver cirrhosis and may contribute to disease progression. Therefore, this thesis aims to investigate the role of the intestine in patients with compensated cirrhosis, paying attention to both the small and large intestine, with specific emphasis on the epithelial barrier function.

In the search for non-invasive tests, we address the role of exhaled breath analyses for the diagnosis of chronic liver diseases, especially cirrhosis at an early stage, as this is important to prevent progression towards end stage, complicated, liver disease.

\section{The role of the intestine}

Chapter 2 provides an overview of the current knowledge regarding the intestinal epithelial barrier function in patients with chronic liver diseases and cirrhosis. Possible contributing factors and mechanisms involved are also discussed. Most studies published so far, focused on small and whole intestinal permeability by measuring the excretion of orally administered test markers in mixed groups of patients with compensated and decompensated cirrhosis. In Chapter 3, a study is presented that investigated the permeability of the small and large intestine in compensated cirrhotic patients compared to healthy controls by using a validated multi-sugar test. In addition, the expression of key TJ genes and proteins in duodenal and sigmoid biopsies is studied.

Oxidative stress is a potential mechanism causing intestinal epithelial cell damage and affecting TJ integrity, and was found to be increased in animal models of cirrhosis and in one human study. Therefore, the occurrence of oxidative stress in the small and large intestine is assessed in the compensated cirrhotic patients and healthy controls and is described in Chapter 4.

As alterations in the intestinal microbiota are an important factor facilitating bacterial translocation, but may also affect for example the epithelial barrier function and immune function, the fecal and mucosa-associated microbiota is investigated in patients with compensated cirrhosis and compared with healthy controls using a validated 16S-23S interspacer (IS) region-based profiling method. In addition, the fecal microbiota is assessed in patients with decompensated cirrhosis. The results of this study are described in Chapter 5.

To further unravel the intestinal involvement in chronic liver diseases, in particular of NAFLD and its progression, an animal study is conducted comparing two mouse models with diet-induced NAFLD. Epithelial barrier function, oxidative stress and smooth muscle contractility are studied in both the small and large intestine, which is described in Chapter 6. 


\section{Non-invasive diagnostics}

Non-invasive alternatives to liver biopsy for diagnosis of chronic liver diseases, especially cirrhosis at an early stage, are important to prevent progression towards end stage complicated cirrhosis. The value of breath tests using ${ }^{13} \mathrm{C}$-labeled probe drugs as non-invasive tests for the evaluation of metabolic liver function is appraised in Chapter 7. In addition, the role of cytochrome P450 enzymes involved in the metabolism of the different probe drugs is discussed. In Chapter 8, the potency of an exhaled VOC profile as a non-invasive biomarker to predict the presence of compensated cirrhosis among a heterogeneous group of patients with chronic liver diseases is investigated and compared with a panel of routine serological markers.

Finally, a general discussion is presented in Chapter 9, summarizing the major findings of all studies and discussing remaining questions and potential implications for clinical practice and future research. 


\section{References}

1. Anthony PP, Ishak KG, Nayak NC, Poulsen HE, Scheuer PJ, Sobin LH. The morphology of cirrhosis: definition, nomenclature, and classification. Bull World Health Organ 1977;55:521-40.

2. Hoyert DL, Xu J. Deaths: Preliminary Data for 2011. National vital statistics reports; vol 61 no 6 . Hyattsville, MD: National Center for Health Statistics. 2011. Available from: http://www.cdc.gov/nchs/ data/nvsr/nvsr61/nvsr61_06.pdf.

3. Blachier M, Leleu H, Peck-Radosavljevic M, Valla DC, Roudot-Thoraval F. The burden of liver disease in Europe: a review of available epidemiological data. J Hepatol 2013;58:593-608.

4. Merion RM. Current status and future of liver transplantation. Semin Liver Dis 2010;30:411-21.

5. Murray KF, Carithers RL, Jr. AASLD practice guidelines: Evaluation of the patient for liver transplantation. Hepatology 2005;41:1407-32.

6. Garcia-Tsao G, Friedman S, Iredale J, Pinzani M. Now there are many (stages) where before there was one: In search of a pathophysiological classification of cirrhosis. Hepatology 2010;51:1445-9.

7. D'Amico G, Garcia-Tsao G, Pagliaro L. Natural history and prognostic indicators of survival in cirrhosis: a systematic review of 118 studies. J Hepatol 2006;44:217-31.

8. Evans LT, Kim WR, Poterucha JJ, Kamath PS. Spontaneous bacterial peritonitis in asymptomatic outpatients with cirrhotic ascites. Hepatology 2003;37:897-901.

9. Cadranel JF, Nousbaum JB, Bessaguet C, et al. Low incidence of spontaneous bacterial peritonitis in asymptomatic cirrhotic outpatients. World J Hepatol 2013;5:104-8.

10. Singal AK, Salameh H, Kamath PS. Prevalence and in-hospital mortality trends of infections among patients with cirrhosis: a nationwide study of hospitalised patients in the United States. Aliment Pharmacol Ther 2014;40:105-12.

11. Pugh RN, Murray-Lyon IM, Dawson JL, Pietroni MC, Williams R. Transection of the oesophagus for bleeding oesophageal varices. Br J Surg 1973;60:646-9.

12. Infante-Rivard C, Esnaola S, Villeneuve JP. Clinical and statistical validity of conventional prognostic factors in predicting short-term survival among cirrhotics. Hepatology 1987;7:660-4.

13. Wiesner R, Edwards E, Freeman R, et al. Model for End-Stage Liver Disease (MELD) and allocation of donor livers. Gastroenterology 2003;124:91-6.

14. Schuppan D, Afdhal NH. Liver cirrhosis. Lancet 2008;371:838-51.

15. Berzigotti A, Garcia-Tsao G, Bosch J, et al. Obesity is an independent risk factor for clinical decompensation in patients with cirrhosis. Hepatology 2011;54:555-61.

16. Ripoll C, Groszmann R, Garcia-Tsao G, et al. Hepatic venous pressure gradient predicts clinical decompensation in patients with compensated cirrhosis. Gastroenterology 2007;133:481-8.

17. Thein $\mathrm{HH}, \mathrm{Yi}$ Q, Dore GJ, Krahn MD. Estimation of stage-specific fibrosis progression rates in chronic hepatitis $C$ virus infection: a meta-analysis and meta-regression. Hepatology 2008;48:418-31.

18. Younossi ZM, Stepanova M, Afendy $M$, et al. Changes in the prevalence of the most common causes of chronic liver diseases in the United States from 1988 to 2008. Clin Gastroenterol Hepatol 2011;9:52430 e1; quiz e60.

19. Vernon G, Baranova A, Younossi ZM. Systematic review: the epidemiology and natural history of nonalcoholic fatty liver disease and non-alcoholic steatohepatitis in adults. Aliment Pharmacol Ther 2011;34:274-85.

20. McCullough AJ. The clinical features, diagnosis and natural history of nonalcoholic fatty liver disease. Clin Liver Dis 2004;8:521-33, viii.

21. Parlesak A, Schäfer C, Schütz T, Bode JC, Bode C. Increased intestinal permeability to macromolecules and endotoxemia in patients with chronic alcohol abuse in different stages of alcohol-induced liver disease. J Hepatol 2000;32:742-7.

22. Bode C, Kolepke R, Schafer K, Bode JC. Breath hydrogen excretion in patients with alcoholic liver disease--evidence of small intestinal bacterial overgrowth. Z Gastroenterol 1993;31:3-7.

23. Wegener M, Schaffstein J, Dilger U, Coenen C, Wedmann B, Schmidt G. Gastrointestinal transit of solid-liquid meal in chronic alcoholics. Dig Dis Sci 1991;36:917-23.

24. Miele L, Valenza V, La Torre G, et al. Increased intestinal permeability and tight junction alterations in nonalcoholic fatty liver disease. Hepatology 2009;49:1877-87. 
25. Soza A, Riquelme A, Gonzalez R, et al. Increased orocecal transit time in patients with nonalcoholic fatty liver disease. Dig Dis Sci 2005;50:1136-40.

26. Bauer TM, Schwacha H, Steinbruckner B, et al. Diagnosis of small intestinal bacterial overgrowth in patients with cirrhosis of the liver: poor performance of the glucose breath hydrogen test. J Hepatol 2000;33:382-6.

27. Chesta J, Defilippi C. Abnormalities in proximal small bowel motility in patients with cirrhosis. Hepatology 1993;17:828-32.

28. Campillo B, Pernet P, Bories PN, Richardet JP, Devanlay M, Aussel C. Intestinal permeability in liver cirrhosis: relationship with severe septic complications. Eur J Gastroenterol Hepatol 1999;11:755-9.

29. Groschwitz KR, Hogan SP. Intestinal barrier function: molecular regulation and disease pathogenesis. J Allergy Clin Immunol 2009;124:3-20; quiz 1-2.

30. Turner JR. Intestinal mucosal barrier function in health and disease. Nat Rev Immunol 2009;9:799-809.

31. Cunningham KE, Turner JR. Myosin light chain kinase: pulling the strings of epithelial tight junction function. Ann N Y Acad Sci 2012;1258:34-42.

32. Purohit V, Bode JC, Bode C, et al. Alcohol, intestinal bacterial growth, intestinal permeability to endotoxin, and medical consequences: summary of a symposium. Alcohol 2008;42:349-61.

33. Ersoz G, Aydin A, Erdem S, Yuksel D, Akarca U, Kumanlioglu K. Intestinal permeability in liver cirrhosis. Eur J Gastroenterol Hepatol 1999;11:409-12.

34. Cariello R, Federico A, Sapone A, et al. Intestinal permeability in patients with chronic liver diseases: Its relationship with the aetiology and the entity of liver damage. Dig Liver Dis 2010;42:200-4.

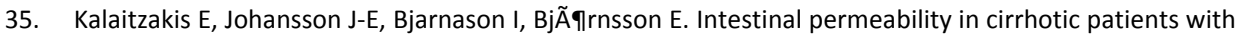
and without ascites. Scand J Gastroenterol. 2006;41:326-30..

36. Pascual S, Such J, Esteban A, et al. Intestinal permeability is increased in patients with advanced cirrhosis. Hepatogastroenterology 2003;50:1482-6.

37. Scarpellini E, Valenza V, Gabrielli M, et al. Intestinal Permeability in Cirrhotic Patients With and Without Spontaneous Bacterial Peritonitis: Is the Ring Closed[quest]. Am J Gastroenterol 2009;105:323-7.

38. Assimakopoulos SF, Tsamandas AC, Tsiaoussis GI, et al. Altered intestinal tight junctions' expression in patients with liver cirrhosis: a pathogenetic mechanism of intestinal hyperpermeability. Eur J Clin Invest 2012;42:439-46.

39. Du Plessis J, Vanheel H, Janssen CE, et al. Activated intestinal macrophages in patients with cirrhosis release NO and IL-6 that may disrupt intestinal barrier function. J Hepatol 2013.

40. Norman K, Pirlich M, Schulzke JD, et al. Increased intestinal permeability in malnourished patients with liver cirrhosis. Eur J Clin Nutr 2012.

41. Elamin EE, Masclee AA, Dekker J, Jonkers DM. Ethanol metabolism and its effects on the intestinal epithelial barrier. Nutr Rev 2013;71:483-99.

42. Moreira AP, Texeira TF, Ferreira AB, Peluzio Mdo C, Alfenas Rde C. Influence of a high-fat diet on gut microbiota, intestinal permeability and metabolic endotoxaemia. Br J Nutr 2012;108:801-9.

43. Schnabl B, Brenner DA. Interactions between the intestinal microbiome and liver diseases. Gastroenterology 2014;146:1513-24.

44. Moustafa AH, Ali EM, Mohamed TM, Abdou HI. Oxidative stress and thyroid hormones in patients with liver diseases. Eur J Intern Med 2009;20:703-8.

45. Zuwala-Jagiello J, Pazgan-Simon M, Simon K, Warwas M. Elevated advanced oxidation protein products levels in patients with liver cirrhosis. Acta Biochim Pol 2009;56:679-85.

46. von Baehr V, Docke WD, Plauth $\mathrm{M}$, et al. Mechanisms of endotoxin tolerance in patients with alcoholic liver cirrhosis: role of interleukin 10, interleukin 1 receptor antagonist, and soluble tumour necrosis factor receptors as well as effector cell desensitisation. Gut 2000;47:281-7.

47. Mortensen C, Andersen O, Krag A, Bendtsen F, Moller S. High-sensitivity C-reactive protein levels predict survival and are related to haemodynamics in alcoholic cirrhosis. Eur J Gastroenterol Hepatol 2012;24:619-26.

48. Banan A, Choudhary S, Zhang Y, Fields JZ, Keshavarzian A. Ethanol-induced barrier dysfunction and its prevention by growth factors in human intestinal monolayers: evidence for oxidative and cytoskeletal mechanisms. J Pharmacol Exp Ther 1999;291:1075-85. 
49. Banan A, Fields JZ, Decker H, Zhang Y, Keshavarzian A. Nitric oxide and its metabolites mediate ethanol-induced microtubule disruption and intestinal barrier dysfunction. J Pharmacol Exp Ther 2000;294:997-1008.

50. Chen $\mathrm{Y}$, Yang $\mathrm{F}$, Lu H, et al. Characterization of fecal microbial communities in patients with liver cirrhosis. Hepatology 2011;54:562-72.

51. Bajaj JS, Hylemon PB, Ridlon JM, et al. Colonic mucosal microbiome differs from stool microbiome in cirrhosis and hepatic encephalopathy and is linked to cognition and inflammation. Am J Physiol Gastrointest Liver Physiol 2012;303:G675-85.

52. Saitoh O, Sugi K, Lojima K, et al. Increased prevalence of intestinal inflammation in patients with liver cirrhosis. World J Gastroenterol 1999;5:391-6.

53. Iwao $\mathrm{T}$, Toyonaga $\mathrm{A}$, Ikegami $\mathrm{M}$, et al. Reduced gastric mucosal blood flow in patients with portalhypertensive gastropathy. Hepatology 1993;18:36-40.

54. Misra V, Misra SP, Dwivedi M, Gupta SC. Histomorphometric study of portal hypertensive enteropathy. Am J Clin Pathol 1997;108:652-7.

55. Xu WH, Wu XJ, Li JS. Influence of portal pressure change on intestinal permeability in patients with portal hypertension. Hepatobiliary Pancreat Dis Int 2002;1:510-4.

56. Assimakopoulos SF, Gogos C, Labropoulou-Karatza C. Could antioxidants be the "magic pill" for cirrhosis-related complications? A pathophysiological appraisal. Med Hypotheses 2011;77:419-23.

57. Ramachandran A, Balasubramanian KA. Intestinal dysfunction in liver cirrhosis: Its role in spontaneous bacterial peritonitis. J Gastroenterol Hepatol 2001;16:607-12.

58. Rao RK, Basuroy S, Rao VU, Karnaky Jr KJ, Gupta A. Tyrosine phosphorylation and dissociation of occludin-ZO-1 and E-cadherin-beta-catenin complexes from the cytoskeleton by oxidative stress. Biochem J 2002;368:471-81.

59. Ramachandran A, Prabhu R, Thomas S, Reddy JB, Pulimood A, Balasubramanian KA. Intestinal mucosal alterations in experimental cirrhosis in the rat: role of oxygen free radicals. Hepatology 2002;35:622-9.

60. Chiva M, Soriano G, Rochat I, et al. Effect of Lactobacillus johnsonii La1 and antioxidants on intestinal flora and bacterial translocation in rats with experimental cirrhosis. J Hepatol 2002;37:456-62.

61. Chiva M, Guarner C, Peralta C, et al. Intestinal mucosal oxidative damage and bacterial translocation in cirrhotic rats. Eur J Gastroenterol Hepatol 2003;15:145-50.

62. Natarajan SK, Ramamoorthy $\mathrm{P}$, Thomas $\mathrm{S}$, et al. Intestinal mucosal alterations in rats with carbon tetrachloride-induced cirrhosis: changes in glycosylation and luminal bacteria. Hepatology 2006;43: 837-46.

63. Assimakopoulos SF, Tsamandas AC, Tsiaoussis GI, et al. Intestinal mucosal proliferation, apoptosis and oxidative stress in patients with liver cirrhosis. Ann Hepatol 2013;12:301-7.

64. Zoetendal EG, Rajilic-Stojanovic M, de Vos WM. High-throughput diversity and functionality analysis of the gastrointestinal tract microbiota. Gut 2008;57:1605-15.

65. Eckburg PB, Bik EM, Bernstein CN, et al. Diversity of the human intestinal microbial flora. Science 2005;308:1635-8.

66. Frank DN, St Amand AL, Feldman RA, Boedeker EC, Harpaz N, Pace NR. Molecular-phylogenetic characterization of microbial community imbalances in human inflammatory bowel diseases. Proc Natl Acad Sci U S A 2007;104:13780-5.

67. Willing BP, Dicksved J, Halfvarson J, et al. A pyrosequencing study in twins shows that gastrointestinal microbial profiles vary with inflammatory bowel disease phenotypes. Gastroenterology 2010;139:1844-54 e1.

68. Ley RE, Turnbaugh PJ, Klein S, Gordon JI. Microbial ecology: human gut microbes associated with obesity. Nature 2006;444:1022-3.

69. Turnbaugh PJ, Hamady M, Yatsunenko T, et al. A core gut microbiome in obese and lean twins. Nature 2009;457:480-4.

70. Larsen N, Vogensen FK, van den Berg FW, et al. Gut microbiota in human adults with type 2 diabetes differs from non-diabetic adults. PLoS One 2010;5:e9085.

71. Qin J, Li Y, Cai Z, et al. A metagenome-wide association study of gut microbiota in type 2 diabetes. Nature 2012;490:55-60.

72. Mouzaki M, Comelli EM, Arendt BM, et al. Intestinal microbiota in patients with nonalcoholic fatty liver disease. Hepatology 2013;58:120-7. 
73. Mutlu EA, Gillevet PM, Rangwala $\mathrm{H}$, et al. Colonic microbiome is altered in alcoholism. Am J Physiol Gastrointest Liver Physiol. 2012;302:G966-78.

74. Chesta J, Silva M, Thompson L, del Canto E, Defilippi C. [Bacterial overgrowth in small intestine in patients with liver cirrhosis]. Rev Med Chil 1991;119:626-32.

75. Bauer TM, Schwacha H, Steinbruckner B, et al. Small intestinal bacterial overgrowth in human cirrhosis is associated with systemic endotoxemia. Am J Gastroenterol 2002;97:2364-70.

76. Bauer TM, Steinbruckner B, Brinkmann FE, et al. Small intestinal bacterial overgrowth in patients with cirrhosis: prevalence and relation with spontaneous bacterial peritonitis. Am J Gastroenterol 2001;96:2962-7.

77. Steed H, Macfarlane GT, Blackett KL, et al. Bacterial translocation in cirrhosis is not caused by an abnormal small bowel gut microbiota. FEMS Immunol Med Microbiol 2011;63:346-54.

78. Wiest R, Lawson M, Geuking M. Pathological bacterial translocation in liver cirrhosis. J Hepatol 2014;60:197-209.

79. Qin N, Yang F, Li A, et al. Alterations of the human gut microbiome in liver cirrhosis. Nature 2014;513:59-64.

80. Wei X, Yan X, Zou D, et al. Abnormal fecal microbiota community and functions in patients with hepatitis B liver cirrhosis as revealed by a metagenomic approach. BMC Gastroenterol 2013;13:175.

81. Bajaj JS, Heuman DM, Hylemon PB, et al. Altered profile of human gut microbiome is associated with cirrhosis and its complications. J Hepatol 2014;60:940-7.

82. Tsochatzis EA, Bosch J, Burroughs AK. Liver cirrhosis. Lancet 2014;383:1749-61.

83. Garcia-Tsao G, Lim JK, Members of Veterans Affairs Hepatitis CRCP. Management and treatment of patients with cirrhosis and portal hypertension: recommendations from the Department of Veterans Affairs Hepatitis C Resource Center Program and the National Hepatitis C Program. Am J Gastroenterol 2009;104:1802-29.

84. Rockey DC, Caldwell SH, Goodman ZD, Nelson RC, Smith AD. Liver biopsy. Hepatology 2009;49:101744.

85. Myers RP, Fong A, Shaheen AA. Utilization rates, complications and costs of percutaneous liver biopsy: a population-based study including 4275 biopsies. Liver Int 2008;28:705-12.

86. Regev A, Berho M, Jeffers $L$, et al. Sampling error and intraobserver variation in liver biopsy in patients with chronic HCV infection. Am J Gastroenterol 2002;97:2614-8.

87. Bedossa P, Dargere D, Paradis V. Sampling variability of liver fibrosis in chronic hepatitis C. Hepatology 2003;38:1449-57.

88. Ratziu V, Charlotte F, Heurtier A, et al. Sampling variability of liver biopsy in nonalcoholic fatty liver disease. Gastroenterology 2005;128:1898-906.

89. Lin ZH, Xin YN, Dong QJ, et al. Performance of the aspartate aminotransferase-to-platelet ratio index for the staging of hepatitis C-related fibrosis: an updated meta-analysis. Hepatology 2011;53:726-36.

90. Vallet-Pichard A, Mallet V, Nalpas B, et al. FIB-4: an inexpensive and accurate marker of fibrosis in HCV infection. comparison with liver biopsy and fibrotest. Hepatology 2007;46:32-6.

91. Patel K, Bedossa P, Castera L. Diagnosis of liver fibrosis: present and future. Semin Liver Dis 2015;35:166-83.

92. Netzer M, Millonig G, OsI M, et al. A new ensemble-based algorithm for identifying breath gas marker candidates in liver disease using ion molecule reaction mass spectrometry. Bioinformatics 2009;25:941-7.

93. Van den Velde S, Nevens F, Van Hee P, van Steenberghe D, Quirynen M. GC-MS analysis of breath odor compounds in liver patients. J Chromatogr B Analyt Technol Biomed Life Sci. 2008;875:344-8.

94. Dadamio J, Van den Velde S, Laleman W, et al. Breath biomarkers of liver cirrhosis. J Chromatogr B Analyt Technol Biomed Life Sci. 2012;905:17-22.

95. Morisco F, Aprea E, Lembo V, et al. Rapid "breath-print" of liver cirrhosis by proton transfer reaction time-of-flight mass spectrometry. A pilot study. PLoS One 2013;8:e59658. 


\section{Chapter 2}

Intestinal epithelial barrier function in liver cirrhosis: an extensive review of the literature

K.E. Pijls

D.M.A.E. Jonkers

E.E. Elamin A.A.M. Masclee G.H. Koek

Liver Int 2013;33:1457-69 


\section{Abstract}

Recent evidence suggests that translocation of bacteria and bacterial products, such as endotoxin from the intestinal lumen into the systemic circulation, is a contributing factor in the pathogenesis of chronic liver diseases and the development of complications in cirrhosis. In addition to alterations in the intestinal microbiota and immune system, dysfunction of the intestinal epithelial barrier may be an important factor facilitating bacterial translocation. This review aims to provide an overview of the current evidence of intestinal epithelial barrier dysfunction in human chronic liver diseases and cirrhosis, and to discuss possible contributing factors and mechanisms.

Data suggest the presence of intestinal epithelial barrier dysfunction in patients with chronic liver diseases, but are more convincing in patients with cirrhosis, especially in those with complications. The barrier dysfunction can result from both direct and indirect effects of etiological factors, such as alcohol and obesity, which can cause chronic liver diseases and ultimately cirrhosis. On the other hand characteristics of cirrhosis itself, including portal hypertension, alterations in the intestinal microbiota, inflammation and oxidative stress can affect barrier function of both small and large intestine and may contribute to the development of complications.

In conclusion, there are indications for intestinal epithelial barrier dysfunction in patients with chronic liver diseases and especially in patients with cirrhosis, which can be caused by various factors affecting both the small and large intestine. 


\section{Introduction}

Cirrhosis is an advanced stage of liver fibrosis accompanied by vascular remodeling. It is the end result of any chronic liver disease for which liver transplantation is the only curative option. The natural history of cirrhosis is usually characterized by an asymptomatic stage of compensated cirrhosis followed by a symptomatic stage of decompensated cirrhosis. In clinical practice, decompensated cirrhosis is defined by complications owing to portal hypertension and impaired liver function, including ascites, variceal hemorrhage, hepatic encephalopathy and jaundice. ${ }^{1}$ According to a report from the National Center for Health Statistics, cirrhosis and chronic liver diseases were the twelfth leading cause of death in the United States in 2011 . $^{2}$

The most common causes of cirrhosis are chronic liver diseases related to alcohol consumption [alcoholic liver disease (ALD)], hepatitis virus infection and obesity and/or other components of the metabolic syndrome [non-alcoholic fatty liver disease (NAFLD)]. Increasing evidence from animal studies indicates that the translocation of bacteria and bacterial products (e.g. endotoxin) from the intestinal lumen into the systemic circulation, resulting in endotoxemia, is a contributing factor in the pathogenesis of several chronic liver diseases by inducing inflammatory changes in the liver. ${ }^{3,4}$ In cirrhosis, bacterial translocation is considered to play an important role in the pathogenesis of its complications. ${ }^{5}$ In addition to alterations in the intestinal microbiota and the immune system, dysfunction of the intestinal epithelial barrier is an important mechanism contributing to enhanced bacterial translocation. ${ }^{6}$ Barrier dysfunction may be affected by putative etiological factors, such as alcohol and obesity, as well as by characteristics of cirrhosis itself. Better understanding of the role of the intestinal epithelial barrier function in human chronic liver diseases and cirrhosis may provide further insight into the pathogenesis of cirrhosis and the development of its complications. The role of bacterial translocation, intestinal microbiota and the immune system [e.g. toll-like receptors (TLRs)] in chronic liver diseases and cirrhosis has recently been reviewed elsewhere. ${ }^{6-9}$

In the current review, we aimed to summarize the available evidence of intestinal epithelial barrier dysfunction in patients with chronic liver diseases, and in patients with compensated and decompensated cirrhosis. In addition, possible contributing factors and mechanisms of intestinal epithelial barrier dysfunction in chronic liver diseases and cirrhosis will be discussed.

\section{The intestinal epithelial barrier}

The intestinal epithelial barrier consists of multiple defense mechanisms, and can mainly be subdivided into an epithelial (i.e. the mucus layer and epithelial cells) and an immunological barrier (i.e. the epithelial secretions and immune cells). In this review we focus on the epithelial barrier, in particular on the single layer of epithelial cells, 
which are connected to each other by junctional complexes. The epithelium on the one hand facilitates absorption of luminal nutrients, water and electrolytes, whereas on the other hand serves as a barrier to prevent translocation of potential harmful substances via transcellular and paracellular transport. ${ }^{10}$ Paracellular transport is regulated by the apical junctional complex, consisting of the tight junction (TJ) and the subjacent adherens junction (AJ). ${ }^{11}$

The TJs seal the paracellular space and form a selective barrier that allows transport via at least two pathways: a high capacity, charge selective pore pathway for small ions and uncharged molecules and a low-capacity leak pathway for larger molecules, regardless of charge. ${ }^{12}$ TJs are considered to be highly dynamic and open and close continuously in response to a variety of stimuli. ${ }^{13}$ They consist of several transmembrane proteins, such as occludin, members of the claudin family, and junction adhesion molecules, as well as cytoplasmic plaque proteins, such as the zonula occludens proteins (i.e. ZO-1, ZO-2 and ZO-3), which connect the transmembrane proteins with the perijunctional actomyosin ring. ${ }^{11,14}$ Contraction of this ring is important in regulating paracellular permeability and is mainly mediated by activation of myosin light chain kinase (MLCK), which phosphorylates myosin II regulatory light chain $(\mathrm{MLC}) .{ }^{11,14}$

The AJs primarily consist of the interaction between the transmembrane protein E-cadherin and the cytoplasmic protein $\beta$-catenin. Similar to the TJs, the AJs are connected with the perijunctional actomyosin ring and thereby may also play a role in regulating paracellular permeability. ${ }^{15}$

\section{Methods to assess the intestinal epithelial barrier}

The intestinal epithelial barrier integrity can be assessed by evaluating the structure and function of the TJs. The morphological structure of the TJs can be studied by freeze fracture electron microscopy using intestinal biopsies. Hereby, the TJs are identified as a network of anastomosing linear fibrils or chains of protein particles, termed TJ strands, which seal adjacent cell membranes. ${ }^{16}$ The number of TJ strands was found to correlate positively with the integrity of the epithelium. ${ }^{17}$ The TJ structure can also be evaluated in thin sections of tissue or epithelial cells using transmission electron microscopy, where membrane fusions and increased electron density between adjacent cells can be observed. ${ }^{18}$ Using the aforementioned techniques, the intercellular space was found to be occluded and therefore the epithelial TJs were considered to be static. However, more advanced techniques using fluorescently labeled TJ proteins, such as fluorescence recovery after photobleaching (FRAP), have demonstrated that, for example, occludin and ZO-1 are highly mobile at the TJ. ${ }^{19,20}$ Furthermore, the expression of TJs and its associated proteins in tissue samples and cell cultures can be assessed on the protein and gene level by means of immunohistochemistry, Western blotting and quantitative polymerase chain reaction $(q P C R) .{ }^{21,22}$ 
In addition to structural data, functional analyses are also often used to evaluate intestinal epithelial barrier integrity. In vivo culture of intestinal epithelial cell monolayers or ex vivo mounting of live excised intestinal mucosa tissue in Ussing chambers with subsequent measurement of transepithelial resistance (TEER) and/or permeation of specific markers (e.g. FITC-dextran or sucralose) across the epithelium are widely accepted. ${ }^{23,24}$ TEER measures the overall epithelial ion fluxes, whereas permeation of most markers points to the paracellular leak pathway. ${ }^{12,25}$ Recently, three dimensional culture of Caco- 2 and T84 cells in synthetic extracellular matrix proteins have also shown to be suitable for the assessment of intestinal epithelial barrier function. ${ }^{26-28}$

In vivo, the most widely accepted method to evaluate TJ function is analyzing the urinary recovery of orally administered inert test markers. Combining two test markers that differ in size and transport (i.e. paracellular or transcellular), enables correction for differences in for example gastric emptying, intestinal transit, dilution by secretions, tissue distribution and renal function. ${ }^{29}$ Examples of these test markers include sugars (monosaccharides and disaccharides, i.e. rhamnose and lactulose, respectively), polyethylene glycols (PEGs: 400, 1000, 4000) and radiolabeled chelates $\left[{ }^{51} \mathrm{Cr}\right.$-ethylenediaminetetraacetic acid $\left({ }^{51} \mathrm{Cr}\right.$-EDTA $),{ }^{99 \mathrm{~m}} \mathrm{Tc}$-diethylenetriaminepentaacetic acid ( ${ }^{99 \mathrm{~m}}$ Tc-DTPA)]. Because of their size, most of these markers reflect the leak pathway. ${ }^{12}$ TJ function can also be evaluated by measuring the presence of intraluminal substances, such as endotoxin and D-lactate, in the systemic circulation. ${ }^{30}$ In addition to loss of TJ integrity, intestinal epithelial barrier dysfunction can result from damage to intestinal epithelial cells. Intestinal cell damage can be assessed noninvasively by measuring endogenous cytosolic enterocyte proteins in plasma or urine, such as intestinal fatty acid binding protein (I-FABP). ${ }^{30}$ Intestinal biopsies provide the possibility for histological evaluation of intestinal epithelial cell damage and apoptosis.

\section{Intestinal epithelial barrier dysfunction in chronic liver diseases and cirrhosis}

Several studies have been published assessing intestinal permeability in patients with chronic liver diseases that may eventually progress towards cirrhosis as well as in patients with cirrhosis in comparison with healthy subjects by measuring the urinary excretion of orally administered test markers.

In Table 2.1, studies in patients with chronic liver diseases are presented, grouped by etiology. Studies performed in chronic alcoholics, of whom at least a subgroup had evidence of liver disease, showed a significant increased gastroduodenal and/or whole intestinal permeability compared to healthy subjects (Table 2.1). ${ }^{31-33}$ 


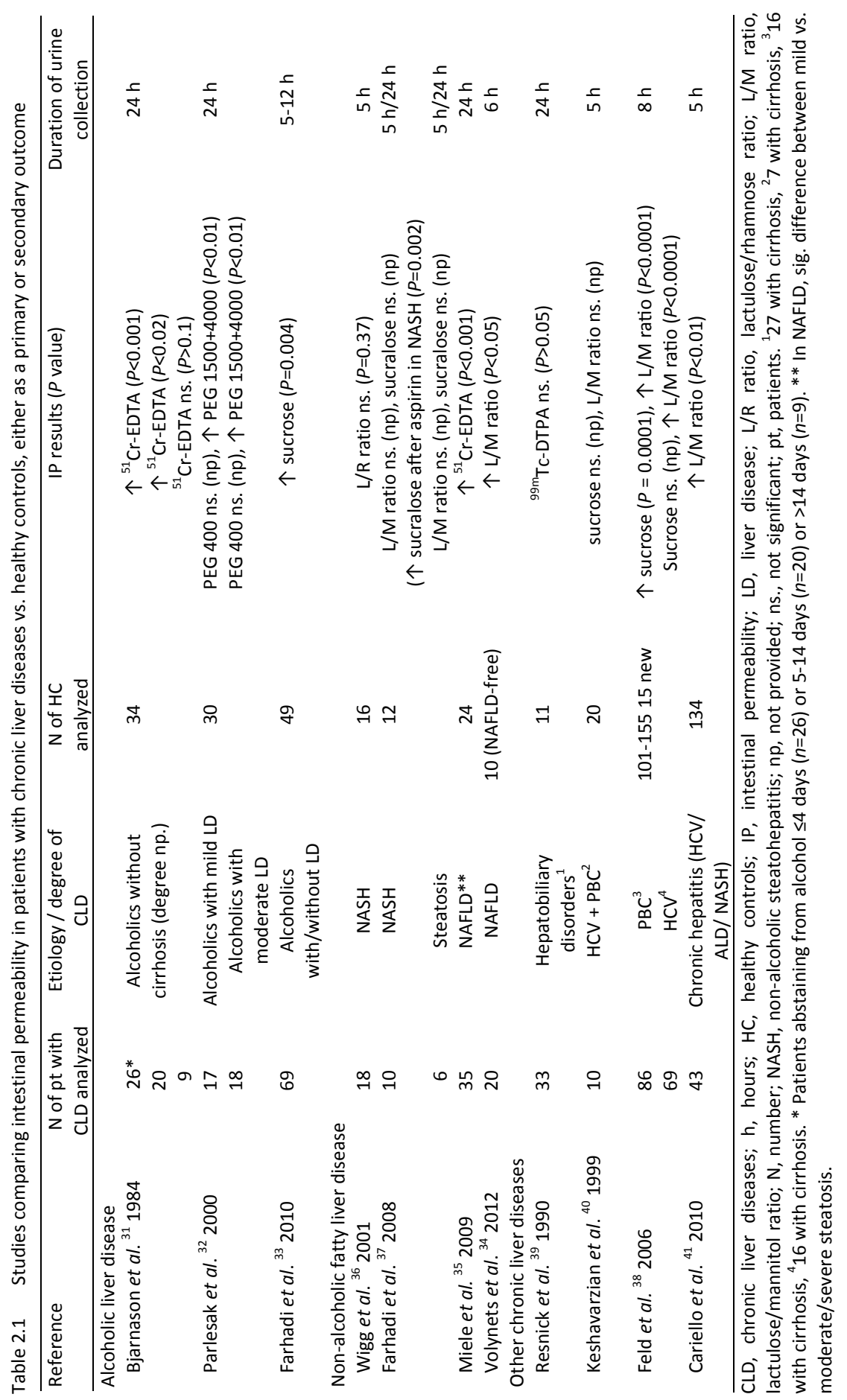


In patients with NAFLD, results are conflicting (Table 2.1). Two studies showed a significant increased small and whole intestinal permeability comparing 20 and 35 NAFLD patients, respectively, with healthy subjects, ${ }^{34,35}$ whereas two smaller studies could not find significant differences. ${ }^{36,37}$ However, the latter study by Farhadi et al. $^{37}$ did observe a significantly increased $0-24 \mathrm{~h}$ and 6-24 $\mathrm{h}$ urinary excretion of sucralose (indicating whole intestinal and large intestinal permeability, respectively) in non-alcoholic steatohepatitis (NASH) patients after an aspirin challenge.

In addition, several studies have assessed intestinal permeability in patients with chronic liver diseases as a result of hepatitis $\mathrm{C}$ virus infection $(\mathrm{HCV}){ }^{38}$ primary biliary cirrhosis $(\mathrm{PBC})^{38}$ or mixed etiologies. ${ }^{39-41}$ Results were not always consistent and no definite conclusion can be drawn from these single studies. Furthermore, in most of them also patients with cirrhosis were included (Table 2.1). ${ }^{38-40}$

Studies assessing intestinal permeability in patients with liver cirrhosis are given in Table 2.2. Most studies found a significantly increased small intestinal, ${ }^{40-54}$ gastroduodenal $^{40,54}$ and whole intestinal ${ }^{32,55-58}$ permeability in patients with cirrhosis when compared with healthy subjects, despite different methods used (e.g. test markers) and patients included (e.g. etiology and severity) (Table 2.2). Studies investigating specifically the large intestine in cirrhosis are scarce; one recent study reported a significantly increased 5-26 $\mathrm{h}$ urinary excretion of sucralose in cirrhotic patients vs. healthy subjects. ${ }^{54}$

As mentioned above, in most studies with cirrhotic patients, mixed groups of patients were included with regard to etiology, severity of cirrhosis and the presence of complications. Most of the studies assessing intestinal permeability in relation to the severity of cirrhosis could not find significant differences among patients with Child $A$, $B$ and $C$ cirrhosis. ${ }^{41-44,47,53-56,59,60}$ However, several of them observed a non-significant increase in permeability as the severity changed from Child $A$ to $B$ and $C$ cirrhosis $^{41,43,44,47,56}$ and three others did find a significantly higher permeability in patients with Child C vs. those with Child A and B cirrhosis. ${ }^{50,57,61}$ Overall, these observations indicate that an increased intestinal permeability may be more pronounced in the most severe degree of cirrhosis.

Studies have also compared intestinal permeability in cirrhotic patients with vs. those without complications, either as primary or secondary outcome (Table 2.3). Most studies did not observe a significant difference in cirrhotic patients with ascites vs. those without ascites, $32,41,43,54,59,60$ but several of them performed these analyses as secondary outcomes only. Four well-designed studies (based on primary outcome and/or large numbers) did find a significantly higher intestinal permeability. ${ }^{47,50,57,61}$ This is further supported by studies showing a significantly increased intestinal permeability in patients with ascites, but not in patients without ascites when compared with healthy controls. ${ }^{47,50,56}$ These findings therefore suggest that especially the presence of ascites is associated with changes in intestinal permeability in cirrhosis. 


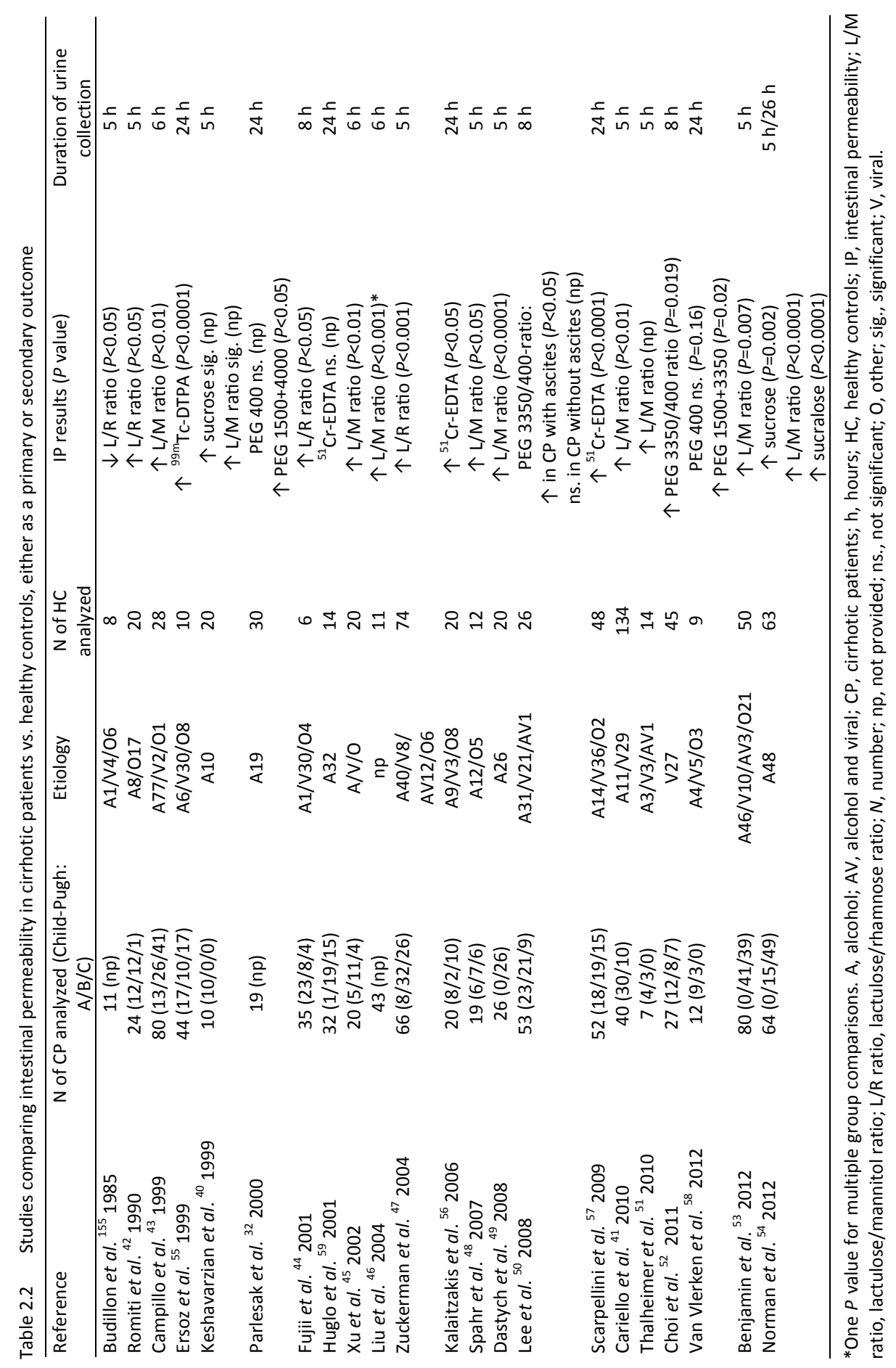




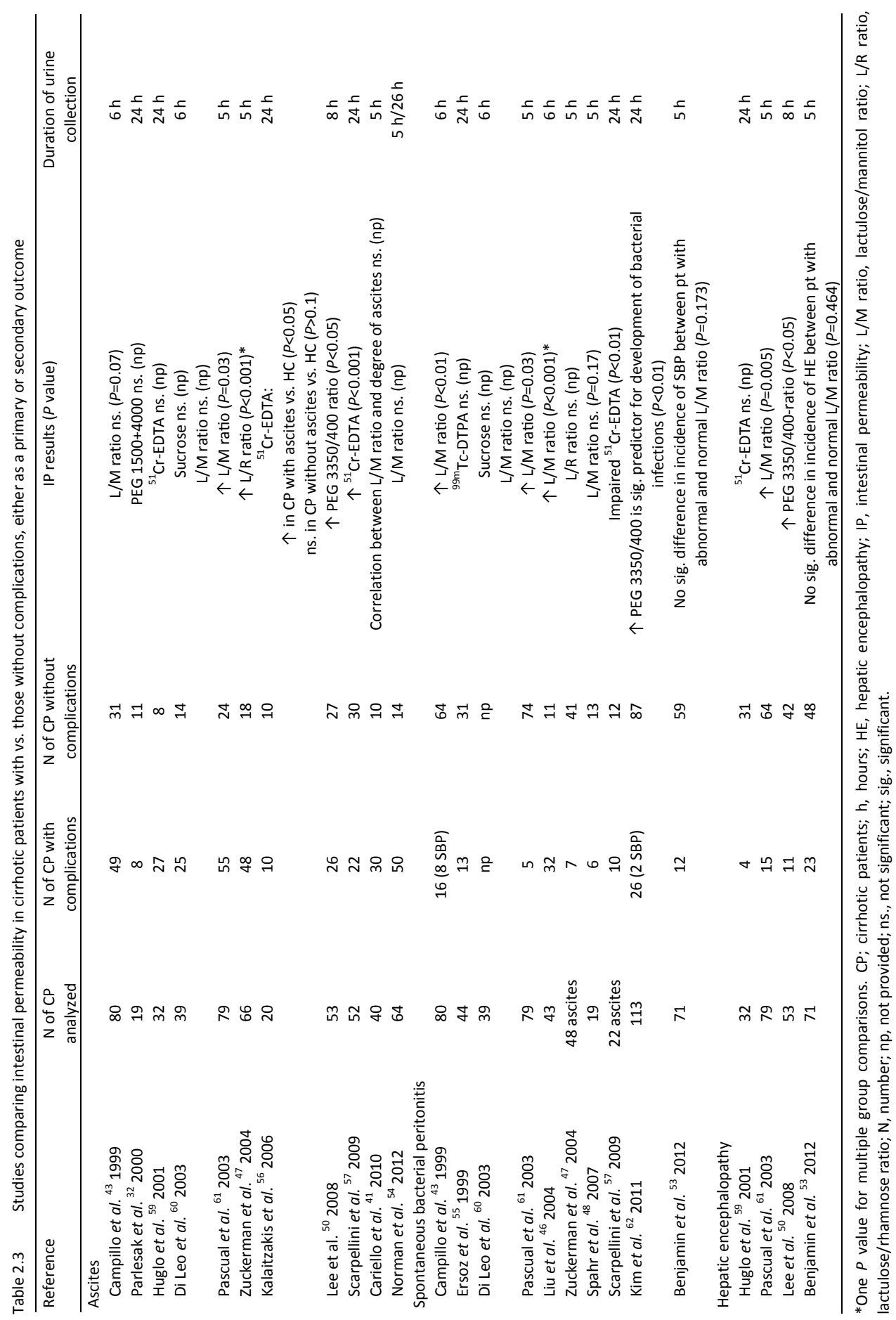


Results on the association between intestinal permeability and spontaneous bacterial peritonitis (SBP) are difficult to interpret because of methodological differences, such as the time of assessing intestinal permeability, and the small number of patients with SBP. ${ }^{43,46-48,53,55,57,60-62}$ Contrasting results have been reported on the association between intestinal permeability and hepatic encephalopathy $(\mathrm{HE}){ }^{50,53,59,61}$

Overall, there are indications for an increased intestinal permeability in patients with chronic liver diseases, especially in patients with ALD and possibly also in patients with NAFLD, but the number of studies is very limited, a variety of test markers is used and in some of them also patients with cirrhosis are included. The majority of studies in cirrhotic patients points to the presence of an increased intestinal permeability. Changes seem to be most pronounced in patients with advanced cirrhosis and/or complications, especially in those with ascites.

It is important to bear in mind that the evaluation of the intestinal epithelial barrier function by the urinary excretion of orally administered test markers has several pitfalls. The possibility of a delayed gastrointestinal transit, differences in volume distribution (for example as a result of the presence of ascites) and renal dysfunction in cirrhotic patients for example, can affect intestinal permeability results when using a single test marker. The urinary excretion ratio of two test markers that differ in size and transport pathway will therefore more accurately reflect intestinal permeability in these patients. Furthermore, medication often used by cirrhotic patients such as NSAIDs or lactulose may influence test results.

Findings of increased endotoxin levels in patients with chronic liver diseases ${ }^{32,34,37}$ and cirrhosis, ${ }^{52,63,64}$ and also the presence of bacterial DNA in blood and ascites in $30-40 \%$ of cirrhotics ${ }^{65-67}$ support the relevance of the increased intestinal permeability observed in these patients. Direct measures of bacterial translocation in humans are too invasive and therefore scarce. One study has been published reporting a significantly increased prevalence of bacterial translocation, defined as the isolation of enteric organisms from mesenteric lymph nodes (MLNs) in patients with Child $C$ cirrhosis compared to Child A and B cirrhosis. ${ }^{68}$

The above-mentioned findings of an increased intestinal permeability suggest an impairment of TJs. However, studies assessing TJ structure in patients with chronic liver diseases and cirrhosis are limited. A small study by Tang et al. ${ }^{69}$ showed that patients with ALD had a significantly reduced protein expression of ZO-1 in sigmoid biopsies compared to healthy subjects, which was accompanied by a significantly higher miR-212 expression in ALD patients. The mRNA expression of ZO-1, however, was not significantly different between both groups. Protein expression of ZO-1 was also found to be significantly lower in duodenal biopsies of NAFLD patients than in healthy subjects. ${ }^{35}$ In addition, in this latter study intestinal permeability was increased.

Cirrhotic patients with and without ascites were studied by Assimakopoulos et al. ${ }^{63}$ They showed a significantly reduced expression of the TJ proteins occludin and claudin1 in duodenal biopsies of the total patient group compared to healthy controls and this 
correlated inversely with the increased plasma endotoxin levels. In addition, the cirrhotic patients with ascites showed a significantly reduced expression of occludin and claudin-1 compared to those without ascites. Recently, increased duodenal protein levels of claudin-2, and a decreased TEER and increased permeability was detected in duodenal biopsies mounted in Ussing chambers from patients with decompensated cirrhosis. ${ }^{70}$ However, no differences in mRNA and proteins levels of occludin, claudin-1, ZO-1 and connexion-43 were found between the cirrhotic patients and controls. Application of electron microscopy in this study, further showed an intact epithelial barrier in patients with decompensated cirrhosis, suggesting that the epithelial barrier in cirrhosis is functionally altered, but structurally normal. ${ }^{70}$ In contrast with the latter finding, Such et $a .^{71}$ found distended intercellular spaces with morphologically intact TJs in duodenal biopsies of six cirrhotic patients with complications.

An increased intestinal permeability may also be related to epithelial cell damage. Histological changes in the intestinal mucosa have been reported in ALD patients, such as villous atrophy, increase in lamina propria infiltrate and intraepithelial lymphocytes, as well as changes in the cellular functions (i.e. brush border, membrane and cellular enzymes). ${ }^{72}$ Changes were also found in the intestinal mucosa of cirrhotic patients with portal hypertension and included shortened and wider microvilli, a decreased villus/crypt ratio, edema of lamina propria, fibromuscular hyperplasia, thickened muscularis mucosae, increased apoptosis and capillary abnormalities. ${ }^{71,73-75}$

Although differences in TJ structure and expression supporting functional permeability analyses have been demonstrated in cirrhotic patients, overall conclusions on specific TJ proteins or subgroups of patients cannot be drawn due to methodological differences and the relatively small number of studies.

\section{Possible contributing factors and mechanisms of intestinal epithelial barrier dysfunction in chronic liver diseases and cirrhosis}

\section{Non-alcoholic fatty liver disease; obesity}

Non-alcoholic fatty liver disease is a common cause of chronic liver disease in developed countries that can progress to cirrhosis. An increase in intestinal permeability has been observed in some studies with NAFLD patients, but not in all (Table 2.1). However, an increased intestinal permeability in animal models and elevated endotoxin levels in both animals and NAFLD patients, ${ }^{34,37,76-79}$ further support the role of a decreased barrier function.

The majority of patients with NAFLD are obese, ${ }^{80}$ and though two small studies could not find a significant difference in intestinal permeability by the urinary excretion of sugars between obese subjects and lean controls, ${ }^{81,82}$ Moreno-Navarrete et al. ${ }^{83}$ 
recently showed that circulating zonulin levels were significantly increased in obese subjects and considered this to be a marker of intestinal permeability. Furthermore, evidence from animal models of obesity clearly shows an increased intestinal permeability, changes in the expression of TJ proteins/genes and elevated endotoxin levels. ${ }^{3,84-86}$

Several possible factors may contribute to the barrier dysfunction in obesity. Results from both animal and human studies have suggested that especially the diet (e.g. highfat intake) itself played a role ${ }^{87-92}$ for example, by altering intestinal microbiota composition. $^{92,93}$

In addition, obesity is considered a state of chronic, low-grade inflammation, characterized by increased production of pro-inflammatory cytokines and adipokines. The systemic inflammation may lead to inflammation and epithelial barrier dysfunction of the small and/or large intestine, as TNF- $\alpha$ and IFN- $-\gamma$, for example, have been shown to increase permeability by disrupting TJ integrity in vitro. ${ }^{94-96}$

Finally, both quantitative and qualitative alterations of the intestinal microbiota have been reported in obesity. ${ }^{97}$ For example, small intestinal bacterial overgrowth (SIBO) has been shown in some obese subjects ${ }^{98,99}$ and although not confirmed in all studies, ${ }^{100,101}$ a shift in the fecal microbiota composition favoring the presence of Firmicutes over Bacteroidetes has been found in obese subjects ${ }^{102}$ and in animal models of obesity. ${ }^{103,104}$ Changes in intestinal microbiota (mainly SIBO) have also been reported in NAFLD patients. ${ }^{105}$

The microbial alterations can affect the intestinal epithelial barrier function indirectly for example via increased endotoxin levels ${ }^{106}$ or directly by altering TJ expression and integrity. ${ }^{107,108}$ The association between intestinal microbiota, intestinal epithelial barrier function and obesity is further supported by intervention studies in mice, showing that modulation of the intestinal microbiota composition with prebiotic and/or antibiotic treatment improved intestinal permeability, changed expression of TJ proteins/genes and reduced endotoxin levels. ${ }^{84,109}$ However, Fouts et al. ${ }^{110}$ showed that increased intestinal permeability and bacterial translocation can also be independent of changes in the intestinal microbiota.

\section{Alcoholic liver disease; alcohol}

Alcohol abuse is one of the major causes of chronic liver disease. In the United States, almost $50 \%$ of all deaths from cirrhosis and chronic liver diseases were found to be attributed to ALD in 2011 . $^{2}$

An increase in intestinal permeability has been found in chronic alcoholics with and without liver disease, ${ }^{31-33,111}$ as well as in non-alcoholics after administration of a single dose of alcohol. ${ }^{112,113}$ Furthermore, endotoxin levels were found to be higher in patients with different stages of ALD than in healthy subjects. ${ }^{32,64,114}$ Several animal studies further support that alcohol can increase intestinal permeability and induce endotoxemia, subsequently leading to liver injury. ${ }^{4,115,116}$ Not only alcohol itself but 
also its oxidative and non-oxidative metabolites, such as acetaldehyde and fatty acid ethyl esters, respectively, may reach the small as well as the large intestine and can contribute to intestinal epithelial barrier dysfunction by disrupting TJ and AJ integrity through several mechanisms involving, for example, MLCK activation and increased generation of reactive oxygen species (ROS). ${ }^{117}$

They may also impair the intestinal barrier function by inducing direct cell damage. ${ }^{118,119}$ Finally, recent evidence found alcohol-induced changes in the intestinal microbiota, ${ }^{120,121}$ which were associated with intestinal epithelial barrier dysfunction. $^{122-124}$

The increased intestinal permeability observed in patients with cirrhosis can be the result of the (on-going) effects of obesity and alcohol. On the other hand, cirrhosis itself can also lead to barrier dysfunction via the effects of portal hypertension, the intestinal microbiota, inflammation and oxidative stress, each of which will be discussed below.

\section{Portal hypertension}

Portal hypertension is a severe consequence of cirrhosis and may lead to ascites, variceal hemorrhage and hepatic encephalopathy. It results from an increased intrahepatic vascular resistance, mainly caused by architectural distortion of the liver secondary to fibrous tissue and regenerative nodules, and an increased portal blood inflow secondary to splanchnic vasodilatation. Splanchnic vasodilatation and the consequent decrease in effective arterial blood volume lead to activation of neurohumoral systems (i.e. renin-angiotensin aldosterone, sympathic nervous system and antidiuretic hormone), which are responsible for the elevated plasma volume. ${ }^{125}$ The latter has been suggested to cause dilatation of epithelial intercellular spaces, probably through the formation of intestinal wall edema, which thereby may alter TJ integrity and increase intestinal permeability. ${ }^{45,71,126}$ Histological studies did show dilatation of the intercellular spaces ${ }^{71,126}$ and edema of the lamina propria in cirrhotic patients with portal hypertension. ${ }^{73}$

Several studies have shown a significantly higher gastroduodenal and/or intestinal permeability in cirrhotic patients with endoscopic signs of portal hypertension than in those without. ${ }^{41,60,127,128}$ The portal pressure was found to correlate significantly with intestinal permeability, and placement of a transjugular intrahepatic portosystemic shunt resulted in a significant decrease of both portal pressure and intestinal permeability in cirrhotic patients. ${ }^{45}$ Consistent with these findings, Reiberger et al. ${ }^{129}$ recently reported a significant correlation between portal pressure and gastroduodenal and intestinal permeability, plasma levels of LPS-binding protein and IL-6 in cirrhotic patients with portal hypertension. The significant improvement of intestinal permeability and bacterial translocation after treatment with non-selective beta-blockers in these patients further supports a role for portal hypertension in affecting intestinal barrier function. ${ }^{129}$ 
A disturbed microcirculation of the intestinal mucosa could lead to an increase in intestinal permeability. ${ }^{45,129}$ Another possible mechanism is nitric oxide (NO), which has been proposed to play a key role in the hemodynamic changes associated with cirrhosis and portal hypertension, ${ }^{130}$ but can also increase intestinal permeability. ${ }^{131}$

\section{The intestinal microbiota}

As stated before, alterations in the intestinal microbiota are an important factor, which can affect TJ integrity and facilitate bacterial translocation. SIBO is a frequent finding in cirrhotic patients when using the glucose-hydrogen breath test and/or culture of jejunal aspirates and has partly been attributed to an impaired intestinal motility. ${ }^{125}$ However, Steed et al. ${ }^{132}$ reported no evidence of bacterial overgrowth by real-time qPCR, except for enterococci, in the duodenal mucosa of cirrhotic patients, and suggested to shift focus to the large intestine. In fecal samples of cirrhotic patients, significantly increased numbers of Escherichia coli and Staphylococcus spp. were found when compared with healthy controls. ${ }^{133}$ Studies using $16 \mathrm{~S}$ rRNA based molecular methods, also showed differences in fecal microbiota composition between cirrhotic patients and healthy controls, including for example a significant increase in the numbers of Enterobacteriaceae. ${ }^{134-137}$ This is in line with $E$. coli being a major cause of SBP and urinary tract infections in these patients. ${ }^{138}$ Recently, the mucosal microbiota composition of the sigmoid of cirrhotic patients was also found to be significantly different from healthy controls (with a lower abundance of potentially beneficial and higher abundance of potentially pathogenic genera). ${ }^{139}$ The difference was most prominent in patients with $\mathrm{HE}$.

\section{Inflammation and oxidative stress}

Cirrhosis is associated with systemic inflammation as shown by elevated levels of inflammatory cytokines, NO and its metabolites in blood of these patients. ${ }^{41,140-144}$ Consequently, this may lead to inflammation and epithelial barrier dysfunction in the small and/or large intestine. Evidence of intestinal inflammation in cirrhotic patients is still limited, but increased fecal concentrations of polymorphonuclear elastase ${ }^{145}$ and calprotectin ${ }^{146}$ have been reported.

Oxidative stress plays also a role in the systemic inflammation and is increased in cirrhosis. It has been hypothesized that inducers of oxidative stress produced in the liver, may cause intestinal oxidative stress upon transferal to the intestine via bile excretion and blood circulation. ${ }^{147,148}$ The decreased antioxidant status observed in cirrhotic patients, ${ }^{149}$ may further facilitate intestinal oxidative stress. Studies in cirrhotic rats have indeed shown evidence of oxidative stress in the small and large intestine, ${ }^{150-153}$ but data in cirrhotic patients are still lacking. Intestinal oxidative stress can impair epithelial barrier function by inducing direct cell damage and/or disrupting TJ and/or AJ integrity. ${ }^{148,154}$ 


\section{Conclusion}

Dysfunction of intestinal epithelial barrier may enhance the risk of translocation of bacteria and bacterial products into the systemic circulation, and thereby contribute to the pathogenesis of chronic liver diseases, cirrhosis, and the development of complications.

Current evidence indicates the presence of intestinal epithelial barrier dysfunction in patients with chronic liver diseases, but is more convincing in patients with cirrhosis, especially in those with complications. Most evidence is derived from studies using the urinary excretion of orally administered test markers (mainly sugars), focusing on small and whole intestinal permeability. However, it should be noted that the increased permeability in these studies does not necessarily prove the occurrence of bacterial translocation, which most likely also involves other mechanisms. Furthermore, reliable analyses of bacterial translocation in humans are difficult and often based on indirect measurements. Additional studies investigating both TJ structure and function of the small and large intestine in patients with cirrhosis are necessary to clarify whether the impaired barrier function is supported by structural TJ alterations, epithelial cell damage and/or bacterial translocation.

Dysfunction of the intestinal epithelial barrier in cirrhosis could be present because of the on-going effects of etiological factors that can cause chronic liver diseases and ultimately cirrhosis, such as obesity and alcohol (Figure 2.1). Clinical and experimental evidence suggests that both can affect barrier function directly and indirectly through mechanisms involving alterations of the intestinal microbiota, inflammation and oxidative stress. Cirrhosis itself is associated with portal hypertension as well as alterations in the intestinal microbiota, chronic on-going inflammation and oxidative stress, each of which can affect barrier function in both small and large intestine and may contribute to the development of complications (Figure 2.1).

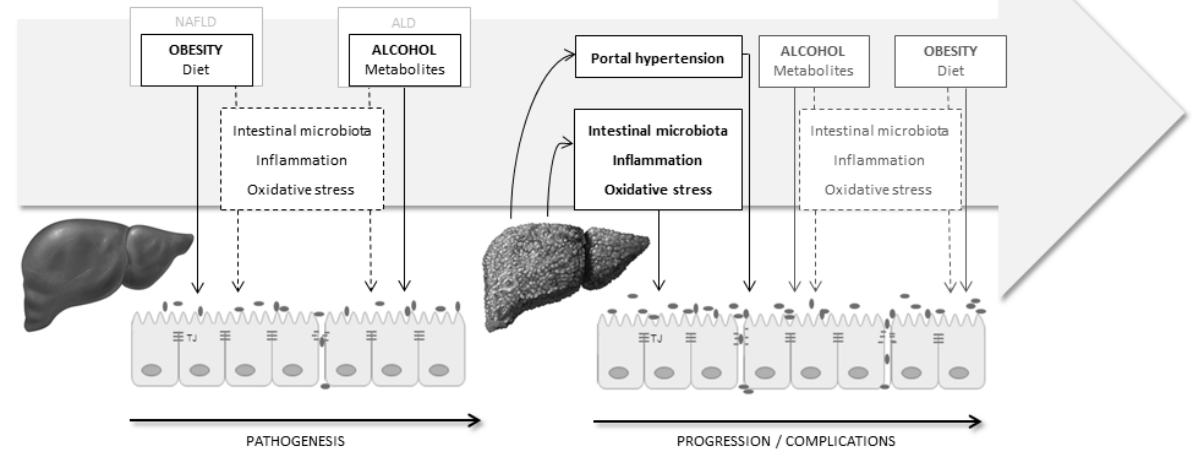

Figure 2.1 Possible contributing factors and mechanisms of intestinal epithelial barrier dysfunction in chronic liver diseases and cirrhosis. 
In conclusion, there are indications for intestinal epithelial barrier dysfunction in patients with chronic liver diseases and especially in patients with cirrhosis, which can be caused by various factors affecting both the small and large intestine and may contribute to the development and progression of these liver diseases. 


\section{References}

1. Garcia-Tsao G, Friedman S, Iredale J, Pinzani M. Now there are many (stages) where before there was one: In search of a pathophysiological classification of cirrhosis. Hepatology 2010;51:1445-9.

2. Hoyert DL, Xu J. Deaths: Preliminary Data for 2011. National vital statistics reports; vol 61 no 6. Hyattsville, MD: National Center for Health Statistics. 2011. Available from: http://www.cdc.gov/ nchs/data/nvsr/nvsr61/nvsr61_06.pdf.

3. Brun P, Castagliuolo I, Di Leo V, et al. Increased intestinal permeability in obese mice: new evidence in the pathogenesis of nonalcoholic steatohepatitis. Am J Physiol Gastrointest Liver Physiol 2007;292:G518-25.

4. Keshavarzian A, Farhadi A, Forsyth $C B$, et al. Evidence that chronic alcohol exposure promotes intestinal oxidative stress, intestinal hyperpermeability and endotoxemia prior to development of alcoholic steatohepatitis in rats. J Hepatol 2009;50:538-47.

5. Wiest R, Garcia-Tsao G. Bacterial translocation (BT) in cirrhosis. Hepatology 2005;41:422-33.

6. Bellot $P$, Frances $R$, Such J. Pathological bacterial translocation in cirrhosis: pathophysiology, diagnosis and clinical implications. Liver Int 2013;33:31-9.

7. Seki E, Schnabl B. Role of innate immunity and the microbiota in liver fibrosis: crosstalk between the liver and gut. J Physiol 2012;590:447-58.

8. Pinzone MR, Celesia BM, Di Rosa M, Cacopardo B, Nunnari G. Microbial translocation in chronic liver diseases. Int J Microbiol 2012;2012:694629.

9. Quigley EM, Stanton C, Murphy EF. The gut microbiota and the liver. Pathophysiological and clinical implications. J Hepatol 2013;58:1020-7.

10. Groschwitz KR, Hogan SP. Intestinal barrier function: molecular regulation and disease pathogenesis. J Allergy Clin Immunol 2009;124:3-20; quiz 1-2.

11. Turner JR. Intestinal mucosal barrier function in health and disease. Nat Rev Immunol 2009;9:799-809.

12. Shen L, Weber CR, Raleigh DR, Yu D, Turner JR. Tight junction pore and leak pathways: a dynamic duo. Annu Rev Physiol 2011;73:283-309.

13. Arrieta MC, Bistritz L, Meddings JB. Alterations in intestinal permeability. Gut 2006;55:1512-20.

14. Van Itallie CM, Anderson JM. The molecular physiology of tight junction pores. Physiology (Bethesda) 2004;19:331-8.

15. Nusrat A, Turner JR, Madara JL. Molecular physiology and pathophysiology of tight junctions. IV. Regulation of tight junctions by extracellular stimuli: nutrients, cytokines, and immune cells. Am J Physiol Gastrointest Liver Physiol 2000;279:G851-7.

16. Staehelin LA, Mukherjee TM, Williams AW. Freeze-etch appearance of the tight junctions in the epithelium of small and large intestine of mice. Protoplasma 1969;67:165-84.

17. Claude P, Goodenough DA. Fracture faces of zonulae occludentes from "tight" and "leaky" epithelia. J Cell Biol 1973;58:390-400.

18. Goodenough DA, Revel JP. A fine structural analysis of intercellular junctions in the mouse liver. J Cell Biol 1970;45:272-90.

19. Shen L, Weber CR, Turner JR. The tight junction protein complex undergoes rapid and continuous molecular remodeling at steady state. J Cell Biol 2008;181:683-95.

20. Riesen FK, Rothen-Rutishauser B, Wunderli-Allenspach H. A ZO1-GFP fusion protein to study the dynamics of tight junctions in living cells. Histochem Cell Biol 2002;117:307-15.

21. Tang VW. Proteomic and bioinformatic analysis of epithelial tight junction reveals an unexpected cluster of synaptic molecules. Biol Direct 2006;1:37.

22. Noth R, Lange-Grumfeld J, Stuber E, et al. Increased intestinal permeability and tight junction disruption by altered expression and localization of occludin in a murine graft versus host disease model. BMC Gastroenterol 2011;11:109.

23. Rao RK. Acetaldehyde-induced increase in paracellular permeability in Caco-2 cell monolayer. Alcohol Clin Exp Res 1998;22:1724-30.

24. Ferrier L, Berard F, Debrauwer L, et al. Impairment of the intestinal barrier by ethanol involves enteric microflora and mast cell activation in rodents. Am J Pathol 2006;168:1148-54.

25. Hidalgo IJ. Cultured intestinal epithelial cell models. Pharm Biotechnol 1996;8:35-50. 
26. Juuti-Uusitalo K, Klunder $\mathrm{L}$, Sjollema KA, et al. Differential effects of TNF (TNFSF2) and IFN-gamma on intestinal epithelial cell morphogenesis and barrier function in three-dimensional culture. PLoS One 2011;6:e22967.

27. Elamin $E$, Jonkers $D$, Juuti-Uusitalo $K$, et al. Effects of ethanol and acetaldehyde on tight junction integrity: in vitro study in a three dimensional intestinal epithelial cell culture model. PLoS One 2012; 7:e35008.

28. Elamin E, Masclee A, Juuti-Uusitalo K, et al. Fatty Acid Ethyl Esters Induce Intestinal Epithelial Barrier Dysfunction via a Reactive Oxygen Species-Dependent Mechanism in a Three-Dimensional Cell Culture Model. PLoS One 2013;8:e58561.

29. Bjarnason I, MacPherson A, Hollander D. Intestinal permeability: an overview. Gastroenterology 1995;108:1566-81.

30. Grootjans J, Thuijls G, Verdam F, Derikx JP, Lenaerts K, Buurman WA. Non-invasive assessment of barrier integrity and function of the human gut. World J Gastrointest Surg 2010;2:61-9.

31. Bjarnason I, Peters TJ, Wise RJ. The leaky gut of alcoholism: possible route of entry for toxic compounds. Lancet 1984;1:179-82.

32. Parlesak A, Schäfer C, Schütz T, Bode JC, Bode C. Increased intestinal permeability to macromolecules and endotoxemia in patients with chronic alcohol abuse in different stages of alcohol-induced liver disease. J Hepatol 2000;32:742-7.

33. Farhadi A, Keshavarzian A, Kwasny MJ, et al. Effects of aspirin on gastroduodenal permeability in alcoholics and controls. Alcohol 2010;44:447-56.

34. Volynets V, Kuper MA, Strahl S, et al. Nutrition, intestinal permeability, and blood ethanol levels are altered in patients with nonalcoholic fatty liver disease (NAFLD). Dig Dis Sci 2012;57:1932-41.

35. Miele L, Valenza V, La Torre G, et al. Increased intestinal permeability and tight junction alterations in nonalcoholic fatty liver disease. Hepatology 2009;49:1877-87.

36. Wigg AJ, Roberts-Thomson IC, Dymock RB, McCarthy PJ, Grose RH, Cummins AG. The role of small intestinal bacterial overgrowth, intestinal permeability, endotoxaemia, and tumour necrosis factor alpha in the pathogenesis of non-alcoholic steatohepatitis. Gut 2001;48:206-11.

37. Farhadi A, Gundlapalli S, Shaikh M, et al. Susceptibility to gut leakiness: a possible mechanism for endotoxaemia in non-alcoholic steatohepatitis. Liver Int 2008;28:1026-33.

38. Feld J, Meddings J, Heathcote E. Abnormal Intestinal Permeability in Primary Biliary Cirrhosis. Dig Dis Sci 2006;51:1607-13.

39. Resnick RH, Royal H, Marshall W, Barron R, Werth T. Intestinal permeability in gastrointestinal disorders. Use of oral [99mTc]DTPA. Dig Dis Sci 1990;35:205-11.

40. Keshavarzian A, Holmes EW, Patel M, Iber F, Fields JZ, Pethkar S. Leaky gut in alcoholic cirrhosis: a possible mechanism for alcohol-induced liver damage. Am J Gastroenterol 1999;94:200-7.

41. Cariello R, Federico A, Sapone A, et al. Intestinal permeability in patients with chronic liver diseases: Its relationship with the aetiology and the entity of liver damage. Dig Liver Dis 2010;42:200-4.

42. Romiti A, Merli M, Martorano $M$, et al. Malabsorption and nutritional abnormalities in patients with liver cirrhosis. Ital J Gastroenterol 1990;22:118-23.

43. Campillo B, Pernet P, Bories PN, Richardet JP, Devanlay M, Aussel C. Intestinal permeability in liver cirrhosis: relationship with severe septic complications. Eur J Gastroenterol Hepatol 1999;11:755-9.

44. Fujii T, Seki T, Maruoka M, et al. Lactulose-L-rhamnose intestinal permeability test in patients with liver cirrhosis. Hepatol Res 2001;19:158-69.

45. Xu WH, Wu XJ, Li JS. Influence of portal pressure change on intestinal permeability in patients with portal hypertension. Hepatobiliary Pancreat Dis Int 2002;1:510-4.

46. Liu H, Zhang S, Yu A, et al. Studies on intestinal permeability of cirrhotic patients by analysis lactulose and mannitol in urine with HPLC/RID/MS. Bioorg Med Chem Lett 2004;14:2339-44.

47. Zuckerman MJ, Menzies IS, $\mathrm{Ho} \mathrm{H}$, et al. Assessment of Intestinal Permeability and Absorption in Cirrhotic Patients with Ascites Using Combined Sugar Probes. Dig Dis Sci 2004;49:621-6.

48. Spahr L, Bresson-Hadni S, Amann P, et al. Allopurinol, oxidative stress and intestinal permeability in patients with cirrhosis: an open-label pilot study. Liver Int 2007;27:54-60.

49. Dastych M, Dastych M, Jr., Novotna H, Cihalova J. Lactulose/mannitol test and specificity, sensitivity, and area under curve of intestinal permeability parameters in patients with liver cirrhosis and Crohn's disease. Dig Dis Sci 2008;53:2789-92. 
50. Lee S, Son SC, Han MJ, et al. Increased intestinal macromolecular permeability and urine nitrite excretion associated with liver cirrhosis with ascites. World J Gastroenterol 2008;14:3884-90.

51. Thalheimer U, De lorio F, Capra F, et al. Altered intestinal function precedes the appearance of bacterial DNA in serum and ascites in patients with cirrhosis: a pilot study. Eur J Gastroenterol Hepatol 2010;22:1228-34.

52. Choi Y, Jeon WK, Hwang SJ, et al. The role of the gut barrier function in the pathophysiology of viral liver cirrhosis. Hepatogastroenterology 2011;58:1244-7.

53. Benjamin J, Singla V, Arora I, Sood S, Joshi YK. Intestinal permeability and complications in liver cirrhosis: A prospective cohort study. Hepatol Res 2013;43:200-7.

54. Norman K, Pirlich M, Schulzke JD, et al. Increased intestinal permeability in malnourished patients with liver cirrhosis. Eur J Clin Nutr 2012;66:1116-9.

55. Ersoz G, Aydin A, Erdem S, Yuksel D, Akarca U, Kumanlioglu K. Intestinal permeability in liver cirrhosis. Eur J Gastroenterol Hepatol 1999;11:409-12.

56. Kalaitzakis E, Johansson JE, Bjarnason I, Bjornsson E. Intestinal permeability in cirrhotic patients with and without ascites. Scand J Gastroenterol 2006;41:326-30.

57. Scarpellini E, Valenza V, Gabrielli M, et al. Intestinal Permeability in Cirrhotic Patients With and Without Spontaneous Bacterial Peritonitis: Is the Ring Closed[quest]. Am J Gastroenterol 2009;105: 323-7.

58. van Vlerken LG, Huisman EJ, van Hoek B, et al. Bacterial infections in cirrhosis: role of proton pump inhibitors and intestinal permeability. Eur J Clin Invest 2012;42:760-7.

59. Huglo D, De Botton S, Canva-Delcambre V, et al. Simultaneous determination of pulmonary and intestinal permeability in patients with alcoholic liver cirrhosis. Eur J Nucl Med Mol Imaging 2001; 28:1505-11.

60. Di Leo V, Venturi C, Baragiotta A, Martines D, Floreani A. Gastroduodenal and intestinal permeability in primary biliary cirrhosis. Eur J Gastroenterol Hepatol 2003;15:967-73.

61. Pascual S, Such J, Esteban A, et al. Intestinal permeability is increased in patients with advanced cirrhosis. Hepatogastroenterology 2003;50:1482-6.

62. Kim BI, Kim HJ, Park JH, et al. Increased intestinal permeability as a predictor of bacterial infections in patients with decompensated liver cirrhosis and hemorrhage. J Gastroenterol Hepatol 2011;26:550-7.

63. Assimakopoulos SF, Tsamandas AC, Tsiaoussis GI, et al. Altered intestinal tight junctions' expression in patients with liver cirrhosis: a pathogenetic mechanism of intestinal hyperpermeability. Eur J Clin Invest 2012;42:439-46.

64. Fukui H, Brauner B, Bode JC, Bode C. Plasma endotoxin concentrations in patients with alcoholic and non-alcoholic liver disease: reevaluation with an improved chromogenic assay. J Hepatol 1991;12: $162-9$.

65. Such J, Frances R, Munoz C, et al. Detection and identification of bacterial DNA in patients with cirrhosis and culture-negative, nonneutrocytic ascites. Hepatology 2002;36:135-41.

66. Frances $\mathrm{R}$, Benlloch $\mathrm{S}$, Zapater $\mathrm{P}$, et al. A sequential study of serum bacterial DNA in patients with advanced cirrhosis and ascites. Hepatology 2004;39:484-91.

67. Zapater P, Frances R, Gonzalez-Navajas JM, et al. Serum and ascitic fluid bacterial DNA: a new independent prognostic factor in noninfected patients with cirrhosis. Hepatology 2008;48:1924-31.

68. Cirera I, Bauer TM, Navasa M, et al. Bacterial translocation of enteric organisms in patients with cirrhosis. J Hepatol 2001;34:32-7.

69. Tang $\mathrm{Y}$, Banan A, Forsyth CB, et al. Effect of alcohol on miR-212 expression in intestinal epithelial cells and its potential role in alcoholic liver disease. Alcohol Clin Exp Res 2008;32:355-64.

70. Du Plessis J, Vanheel $\mathrm{H}$, Janssen $\mathrm{CE}$, et al. Activated intestinal macrophages in patients with cirrhosis release NO and IL-6 that may disrupt intestinal barrier function. J Hepatol 2013;58:1125-32.

71. Such J, Guardiola JV, de Juan J, et al. Ultrastructural characteristics of distal duodenum mucosa in patients with cirrhosis. Eur J Gastroenterol Hepatol 2002;14:371-6.

72. Bhonchal S, Nain CK, Prasad KK, et al. Functional and morphological alterations in small intestine mucosa of chronic alcoholics. J Gastroenterol Hepatol 2008;23:e43-8.

73. Misra V, Misra SP, Dwivedi M, Gupta SC. Histomorphometric study of portal hypertensive enteropathy. Am J Clin Pathol 1997;108:652-7.

74. Misra V, Misra SP, Dwivedi M, Singh PA, Kumar V. Colonic mucosa in patients with portal hypertension. J Gastroenterol Hepatol 2003;18:302-8. 
75. Barakat M, Mostafa M, Mahran Z, Soliman AG. Portal hypertensive duodenopathy: clinical, endoscopic, and histopathologic profiles. Am J Gastroenterol 2007;102:2793-802.

76. Jin X, Yu CH, Lv GC, Li YM. Increased intestinal permeability in pathogenesis and progress of nonalcoholic steatohepatitis in rats. World J Gastroenterol 2007;13:1732-6.

77. Li S, Wu WC, He CY, Han Z, Jin DY, Wang L. Change of intestinal mucosa barrier function in the progress of non-alcoholic steatohepatitis in rats. World J Gastroenterol 2008;14:3254-8.

78. Harte AL, da Silva NF, Creely SJ, et al. Elevated endotoxin levels in non-alcoholic fatty liver disease. J Inflamm 2010;7:15.

79. Verdam FJ, Rensen SS, Driessen A, Greve JW, Buurman WA. Novel evidence for chronic exposure to endotoxin in human nonalcoholic steatohepatitis. J Clin Gastroenterol 2011;45:149-52.

80. Youssef $\mathrm{WI}, \mathrm{McC}$ cullough AJ. Steatohepatitis in obese individuals. Best Pract Res Clin Gastroenterol 2002;16:733-47.

81. Brignardello J, Morales P, Diaz E, Romero J, Brunser O, Gotteland M. Pilot study: alterations of intestinal microbiota in obese humans are not associated with colonic inflammation or disturbances of barrier function. Aliment Pharmacol Ther 2010;32:1307-14.

82. Teixeira TF, Souza NC, Chiarello PG, et al. Intestinal permeability parameters in obese patients are correlated with metabolic syndrome risk factors. Clin Nutr 2012;31:735-40.

83. Moreno-Navarrete JM, Sabater M, Ortega F, Ricart W, Fernandez-Real JM. Circulating zonulin, a marker of intestinal permeability, is increased in association with obesity-associated insulin resistance. PLoS One 2012;7:e37160.

84. Cani PD, Bibiloni R, Knauf C, et al. Changes in gut microbiota control metabolic endotoxemia-induced inflammation in high-fat diet-induced obesity and diabetes in mice. Diabetes 2008;57:1470-81.

85. de La Serre CB, Ellis CL, Lee J, Hartman AL, Rutledge JC, Raybould HE. Propensity to high-fat dietinduced obesity in rats is associated with changes in the gut microbiota and gut inflammation. Am $\mathrm{J}$ Physiol Gastrointest Liver Physiol 2010;299:G440-8.

86. Lam YY, Ha CW, Campbell CR, et al. Increased gut permeability and microbiota change associate with mesenteric fat inflammation and metabolic dysfunction in diet-induced obese mice. PLoS One 2012;7:e34233.

87. Suzuki T, Hara H. Dietary fat and bile juice, but not obesity, are responsible for the increase in small intestinal permeability induced through the suppression of tight junction protein expression in LETO and OLETF rats. Nutr Metab (Lond) 2010;7:19.

88. Erridge C, Attina T, Spickett CM, Webb DJ. A high-fat meal induces low-grade endotoxemia: evidence of a novel mechanism of postprandial inflammation. Am J Clin Nutr 2007;86:1286-92.

89. Amar J, Burcelin R, Ruidavets JB, et al. Energy intake is associated with endotoxemia in apparently healthy men. Am J Clin Nutr 2008;87:1219-23.

90. Ghanim H, Abuaysheh S, Sia CL, et al. Increase in plasma endotoxin concentrations and the expression of Toll-like receptors and suppressor of cytokine signaling-3 in mononuclear cells after a high-fat, highcarbohydrate meal: implications for insulin resistance. Diabetes Care 2009;32:2281-7.

91. Laugerette F, Vors C, Geloen A, et al. Emulsified lipids increase endotoxemia: possible role in early postprandial low-grade inflammation. J Nutr Biochem 2011;22:53-9.

92. Serino $M$, Luche $E$, Gres $S$, et al. Metabolic adaptation to a high-fat diet is associated with a change in the gut microbiota. Gut 2012;61:543-53.

93. Moreira AP, Texeira TF, Ferreira AB, Peluzio Mdo C, Alfenas Rde C. Influence of a high-fat diet on gut microbiota, intestinal permeability and metabolic endotoxaemia. Br J Nutr 2012;108:801-9.

94. Bruewer $M$, Luegering $A$, Kucharzik $T$, et al. Proinflammatory cytokines disrupt epithelial barrier function by apoptosis-independent mechanisms. J Immunol 2003;171:6164-72.

95. Bruewer $M$, Utech $M$, Ivanov Al, Hopkins AM, Parkos CA, Nusrat A. Interferon-gamma induces internalization of epithelial tight junction proteins via a macropinocytosis-like process. FASEB J 2005; 19:923-33.

96. Zolotarevsky $Y$, Hecht G, Koutsouris A, et al. A membrane-permeant peptide that inhibits MLC kinase restores barrier function in in vitro models of intestinal disease. Gastroenterology 2002;123:163-72.

97. Shen J, Obin MS, Zhao L. The gut microbiota, obesity and insulin resistance. Mol Aspects Med 2013;34:39-58.

98. Sabate JM, Jouet $P$, Harnois $F$, et al. High prevalence of small intestinal bacterial overgrowth in patients with morbid obesity: a contributor to severe hepatic steatosis. Obes Surg 2008;18:371-7. 
99. Madrid AM, Poniachik J, Quera R, Defilippi C. Small intestinal clustered contractions and bacterial overgrowth: a frequent finding in obese patients. Dig Dis Sci 2011;56:155-60.

100. Schwiertz A, Taras D, Schafer K, et al. Microbiota and SCFA in lean and overweight healthy subjects. Obesity 2010;18:190-5.

101. Duncan SH, Lobley GE, Holtrop G, et al. Human colonic microbiota associated with diet, obesity and weight loss. Int J Obes 2008;32:1720-4.

102. Ley RE, Turnbaugh PJ, Klein S, Gordon JI. Microbial ecology: human gut microbes associated with obesity. Nature 2006;444:1022-3.

103. Ley RE, Backhed F, Turnbaugh P, Lozupone CA, Knight RD, Gordon Jl. Obesity alters gut microbial ecology. Proc Natl Acad Sci U S A 2005;102:11070-5.

104. Turnbaugh PJ, Backhed F, Fulton L, Gordon JI. Diet-induced obesity is linked to marked but reversible alterations in the mouse distal gut microbiome. Cell Host Microbe 2008;3:213-23.

105. Machado MV, Cortez-Pinto H. Gut microbiota and nonalcoholic fatty liver disease. Ann Hepatol 2012;11:440-9.

106. Forsythe RM, Xu DZ, Lu Q, Deitch EA. Lipopolysaccharide-induced enterocyte-derived nitric oxide induces intestinal monolayer permeability in an autocrine fashion. Shock 2002;17:180-4.

107. Karczewski J, Troost FJ, Konings I, et al. Regulation of human epithelial tight junction proteins by Lactobacillus plantarum in vivo and protective effects on the epithelial barrier. Am J Physiol Gastrointest Liver Physiol 2010;298:G851-9.

108. Qin H, Zhang Z, Hang X, Jiang Y. L. plantarum prevents enteroinvasive Escherichia coli-induced tight junction proteins changes in intestinal epithelial cells. BMC Microbiol 2009;9:63.

109. Cani PD, Possemiers S, Van de Wiele T, et al. Changes in gut microbiota control inflammation in obese mice through a mechanism involving GLP-2-driven improvement of gut permeability. Gut 2009;58:1091-103.

110. Fouts DE, Torralba M, Nelson KE, Brenner DA, Schnabl B. Bacterial translocation and changes in the intestinal microbiome in mouse models of liver disease. J Hepatol 2012;56:1283-92.

111. Keshavarzian A, Fields JZ, Vaeth J, Holmes EW. The differing effects of acute and chronic alcohol on gastric and intestinal permeability. Am J Gastroenterol 1994;89:2205-11.

112. Robinson GM, Orrego H, Israel Y, Devenyi P, Kapur BM. Low-molecular-weight polyethylene glycol as a probe of gastrointestinal permeability after alcohol ingestion. Dig Dis Sci 1981;26:971-7.

113. Gotteland M, Cruchet S, Frau V, et al. Effect of acute cigarette smoking, alone or with alcohol, on gastric barrier function in healthy volunteers. Dig Liver Dis 2002;34:702-6.

114. Schafer C, Parlesak A, Schutt C, Bode JC, Bode C. Concentrations of lipopolysaccharide-binding protein, bactericidal/permeability-increasing protein, soluble CD14 and plasma lipids in relation to endotoxaemia in patients with alcoholic liver disease. Alcohol Alcohol 2002;37:81-6.

115. Keshavarzian A, Choudhary S, Holmes EW, et al. Preventing gut leakiness by oats supplementation ameliorates alcohol-induced liver damage in rats. J Pharmacol Exp Ther 2001;299:442-8.

116. Lambert JC, Zhou Z, Wang L, Song Z, McClain CJ, Kang YJ. Prevention of alterations in intestinal permeability is involved in zinc inhibition of acute ethanol-induced liver damage in mice. J Pharmacol Exp Ther 2003;305:880-6.

117. Elamin EE, Masclee AA, Dekker J, Jonkers DM. Ethanol metabolism and its effects on the intestinal epithelial barrier. Nutr Rev 2013;71:483-99.

118. Gottfried EB, Korsten MA, Lieber CS. Alcohol-induced gastric and duodenal lesions in man. Am J Gastroenterol 1978;70:587-92.

119. Asai K, Buurman WA, Reutelingsperger CP, Schutte B, Kaminishi M. Low concentrations of ethanol induce apoptosis in human intestinal cells. Scand J Gastroenterol 2003;38:1154-61.

120. Bode JC, Bode C, Heidelbach R, Durr HK, Martini GA. Jejunal microflora in patients with chronic alcohol abuse. Hepatogastroenterology 1984;31:30-4.

121. Hauge T, Persson J, Danielsson D. Mucosal bacterial growth in the upper gastrointestinal tract in alcoholics (heavy drinkers). Digestion 1997;58:591-5.

122. Mutlu E, Keshavarzian A, Engen P, Forsyth CB, Sikaroodi M, Gillevet P. Intestinal dysbiosis: a possible mechanism of alcohol-induced endotoxemia and alcoholic steatohepatitis in rats. Alcohol Clin Exp Res 2009;33:1836-46.

123. Yan AW, Fouts DE, Brandl J, et al. Enteric dysbiosis associated with a mouse model of alcoholic liver disease. Hepatology 2011;53:96-105. 
124. Mutlu EA, Gillevet PM, Rangwala H, et al. Colonic microbiome is altered in alcoholism. Am J Physiol Gastrointest Liver Physiol 2012;302:G966-78.

125. Garcia-Tsao G, Wiest R. Gut microflora in the pathogenesis of the complications of cirrhosis. Best Pract Res Clin Gastroenterol 2004;18:353-72.

126. Norman DA, Atkins JM, Seelig LL, Jr., Gomez-Sanchez C, Krejs GJ. Water and electrolyte movement and mucosal morphology in the jejunum of patients with portal hypertension. Gastroenterology 1980;79:707-15.

127. Giofre MR, Meduri G, Pallio S, et al. Gastric permeability to sucrose is increased in portal hypertensive gastropathy. Eur J Gastroenterol Hepatol 2000;12:529-33.

128. Senzolo M, Fries W, Buda A, et al. Oral propranolol decreases intestinal permeability in patients with cirrhosis: another protective mechanism against bleeding? Am J Gastroenterol 2009;104:3115-6.

129. Reiberger T, Ferlitsch A, Payer BA, et al. Non-selective betablocker therapy decreases intestinal permeability and serum levels of LBP and IL-6 in patients with cirrhosis. J Hepatol 2013;58:911-21.

130. Wiest R, Groszmann RJ. The paradox of nitric oxide in cirrhosis and portal hypertension: too much, not enough. Hepatology 2002;35:478-91.

131. Han X, Fink MP, Delude RL. Proinflammatory cytokines cause NO*-dependent and -independent changes in expression and localization of tight junction proteins in intestinal epithelial cells. Shock 2003;19:229-37.

132. Steed $\mathrm{H}$, Macfarlane $\mathrm{GT}$, Blackett $\mathrm{KL}$, et al. Bacterial translocation in cirrhosis is not caused by an abnormal small bowel gut microbiota. FEMS Immunol Med Microbiol 2011;63:346-54.

133. Liu Q, Duan ZP, Ha DK, Bengmark S, Kurtovic J, Riordan SM. Synbiotic modulation of gut flora: effect on minimal hepatic encephalopathy in patients with cirrhosis. Hepatology 2004;39:1441-9.

134. Lu H, Wu Z, Xu W, Yang J, Chen Y, Li L. Intestinal microbiota was assessed in cirrhotic patients with hepatitis B virus infection. Intestinal microbiota of HBV cirrhotic patients. Microb Ecol 2011;61: 693-703.

135. Chen Y, Yang F, Lu H, et al. Characterization of fecal microbial communities in patients with liver cirrhosis. Hepatology 2011;54:562-72.

136. Bajaj JS, Ridlon JM, Hylemon PB, et al. Linkage of gut microbiome with cognition in hepatic encephalopathy. Am J Physiol Gastrointest Liver Physiol 2012;302:G168-75.

137. Liu J, Wu D, Ahmed A, et al. Comparison of the gut microbe profiles and numbers between patients with liver cirrhosis and healthy individuals. Curr Microbiol 2012;65:7-13.

138. Tandon P, Garcia-Tsao G. Bacterial infections, sepsis, and multiorgan failure in cirrhosis. Semin Liver Dis 2008;28:26-42.

139. Bajaj JS, Hylemon PB, Ridlon JM, et al. Colonic mucosal microbiome differs from stool microbiome in cirrhosis and hepatic encephalopathy and is linked to cognition and inflammation. Am J Physiol Gastrointest Liver Physiol 2012;303:G675-85.

140. von Baehr V, Docke WD, Plauth M, et al. Mechanisms of endotoxin tolerance in patients with alcoholic liver cirrhosis: role of interleukin 10, interleukin 1 receptor antagonist, and soluble tumour necrosis factor receptors as well as effector cell desensitisation. Gut 2000;47:281-7.

141. Mortensen C, Andersen O, Krag A, Bendtsen F, Moller S. High-sensitivity C-reactive protein levels predict survival and are related to haemodynamics in alcoholic cirrhosis. Eur J Gastroenterol Hepatol 2012;24:619-26.

142. Guarner C, Soriano G, Tomas A, et al. Increased serum nitrite and nitrate levels in patients with cirrhosis: relationship to endotoxemia. Hepatology 1993;18:1139-43.

143. Battista S, Bar F, Mengozzi G, Zanon E, Grosso M, Molino G. Hyperdynamic circulation in patients with cirrhosis: direct measurement of nitric oxide levels in hepatic and portal veins. J Hepatol 1997;26: 75-80.

144. Genesca J, Gonzalez A, Segura R, et al. Interleukin-6, nitric oxide, and the clinical and hemodynamic alterations of patients with liver cirrhosis. Am J Gastroenterol 1999;94:169-77.

145. Saitoh O, Sugi K, Lojima K, et al. Increased prevalence of intestinal inflammation in patients with liver cirrhosis. World J Gastroenterol 1999;5:391-6.

146. Gundling F, Schmidtler F, Hapfelmeier A, et al. Fecal calprotectin is a useful screening parameter for hepatic encephalopathy and spontaneous bacterial peritonitis in cirrhosis. Liver Int 2011;31:1406-15.

147. Ramachandran A, Balasubramanian KA. Intestinal dysfunction in liver cirrhosis: Its role in spontaneous bacterial peritonitis. J Gastroenterol Hepatol 2001;16:607-12. 
148. Assimakopoulos SF, Gogos C, Labropoulou-Karatza C. Could antioxidants be the "magic pill" for cirrhosis-related complications? A pathophysiological appraisal. Med Hypotheses 2011;77:419-23.

149. Zuwala-Jagiello J, Pazgan-Simon M, Simon K, Warwas M. Elevated advanced oxidation protein products levels in patients with liver cirrhosis. Acta Biochim Pol 2009;56:679-85.

150. Ramachandran A, Prabhu R, Thomas S, Reddy JB, Pulimood A, Balasubramanian KA. Intestinal mucosal alterations in experimental cirrhosis in the rat: role of oxygen free radicals. Hepatology 2002;35:622-9.

151. Chiva M, Soriano G, Rochat I, et al. Effect of Lactobacillus johnsonii La1 and antioxidants on intestinal flora and bacterial translocation in rats with experimental cirrhosis. J Hepatol 2002;37:456-62.

152. Chiva M, Guarner C, Peralta C, et al. Intestinal mucosal oxidative damage and bacterial translocation in cirrhotic rats. Eur J Gastroenterol Hepatol 2003;15:145-50.

153. Natarajan SK, Ramamoorthy P, Thomas S, et al. Intestinal mucosal alterations in rats with carbon tetrachloride-induced cirrhosis: changes in glycosylation and luminal bacteria. Hepatology 2006;43: 837-46.

154. Rao RK, Basuroy S, Rao VU, Karnaky Jr KJ, Gupta A. Tyrosine phosphorylation and dissociation of occludin-ZO-1 and E-cadherin-beta-catenin complexes from the cytoskeleton by oxidative stress. Biochem J 2002;368:471-81.

155. Budillon G, Parrilli G, Pacella M, Cuomo R, Menzies IS. Investigation of intestine and liver function in cirrhosis using combined sugar oral loads. J Hepatol 1985;1:513-24. 
44 


\section{Chapter 3}

Large intestinal permeability is increased in patients with compensated liver cirrhosis

K.E. Pijls G.H. Koek

E.E. Elamin H. de Vries A.A.M. Masclee D.M.A.E. Jonkers

Am J Physiol Gastrointest Liver Physiol 2014;306:G147-53 


\section{Abstract}

Intestinal barrier dysfunction, facilitating translocation of bacteria and bacterial products, plays an important role in the pathophysiology of liver cirrhosis and its complications. Increased intestinal permeability has been found in patients with liver cirrhosis, but data on small and large intestinal permeability and tight junctions (TJs) in patients with compensated cirrhosis are scarce. We aimed to investigate both small and large intestinal permeability in patients with stable compensated cirrhosis compared with healthy controls and evaluated the expression of TJ proteins in mucosal biopsies at duodenal and sigmoid level.

Intestinal permeability was assessed in 26 patients with compensated cirrhosis and 27 matched controls using a multi-sugar test. Duodenal and sigmoid biopsies were available from a subgroup for analyses of gene transcription and expression of key TJ proteins by qRT-PCR and ELISA, respectively.

Median 0-5 h urinary sucrose excretion and lactulose/rhamnose ratio were comparable between patients with compensated cirrhosis and controls, whereas 5-24 h urinary sucralose/erythritol ratio was increased in these patients. Downregulation of gene transcription was found for claudin-3 in duodenal biopsies and claudin-4 in sigmoid biopsies, and at the protein level occludin expression was significantly increased in both duodenal and sigmoid biopsies.

This study shows that gastroduodenal and small intestinal permeability are not altered, whereas large intestinal permeability is increased in patients with stable compensated cirrhosis. Only limited alterations were found regarding the expression of TJ proteins in both the small and large intestine. 


\section{Introduction}

Translocation of bacteria or bacterial products from the intestinal lumen into the systemic circulation is considered to play an important role in the development of liver cirrhosis and its complications. ${ }^{1,2}$ Factors facilitating bacterial translocation are alterations in the composition of intestinal microbiota, the immune system and disturbances of the intestinal epithelial barrier. ${ }^{3,4}$

Epithelial integrity is essential to prevent the entry of potentially harmful luminal substances, e.g. bacteria and their products, into the systemic circulation by means of transcellular and paracellular pathways. Although apart from an important role for transcellular bacterial translocation and involvement of a well-functioning chemical barrier (e.g. mucus and defensin secretion) and immune system, an increased paracellular permeability will enhance bacterial translocation. The paracellular pathway is regulated by the tight junctions (TJs), which are components of the apical junctional complex that seal the intercellular spaces. ${ }^{5}$ The TJs are composed of transmembrane proteins, such as occludin and members of the claudin family, and cytoplasmic plaque proteins, including the zonula occludens proteins (i.e. ZO-1, ZO-2 and ZO-3), that link the transmembrane proteins to the actin cytoskeleton. 5

Previous studies have pointed to an increased small and even whole intestinal permeability in patients with compensated and/or decompensated cirrhosis compared to healthy controls, ${ }^{7-10}$ and it seems to be most pronounced in decompensated cirrhosis. ${ }^{11,12}$ It is not completely clear whether the intestinal permeability is also increased in patients with compensated cirrhosis and thereby might be a risk factor for the progression towards decompensated cirrhosis. Studies on intestinal permeability have primarily focused on functional tests by measuring urinary excretion of orally administered test markers. Only two studies with contrasting results have assessed the expression of TJ proteins in duodenal biopsies of patients with cirrhosis. ${ }^{13,14}$ Although recent evidence points towards a role of the microbiota and the damaging effects of alcohol in the large intestine ${ }^{15,16}$ in the pathophysiology of liver cirrhosis, studies investigating the epithelial integrity of the large intestine are scarce. One study, using a single test marker, found an increased large intestinal permeability in patients with cirrhosis. ${ }^{17}$

Aim of the present study was therefore to investigate intestinal permeability, not only of the small intestine, but also of the large intestine in patients with compensated cirrhosis and compare data with those obtained in healthy controls. Permeability was studied by using a multi-sugar test as well as by the expression of TJ proteins in mucosal biopsies of duodenum and sigmoid. 


\section{Materials and methods}

The study has been approved by the Medical Ethics Committee of Maastricht University Medical Center (MUMC), conducted according to the revised version of the Declaration of Helsinki (October 2008, Seoul) and registered at the US National Library of Medicine (http://www.clinicaltrials.gov, NTC01081236). All subjects (patients and healthy volunteers) gave their written informed consent before participation.

\section{Patients}

Patients with stable compensated liver cirrhosis aged between 18 and 75 years were recruited from the outpatient clinic of our department. A diagnosis of cirrhosis was based on liver histology and/or clinical, laboratory, radiological, and/or endoscopic findings. Compensated cirrhosis was defined by the absence of clinically evident complications, i.e. ascites, variceal hemorrhage, hepatic encephalopathy and/or jaundice. Exclusion criteria were malignancy, other chronic gastrointestinal diseases, renal failure, diabetes mellitus requiring insulin therapy, and major abdominal surgery interfering with gastrointestinal function. In the included patients, severity of cirrhosis was assessed according to the Child-Pugh classification and the Model for End Stage Liver Disease (MELD) score. Alcohol consumption was assessed by questionnaire and interview. Portal hypertension was based on the previous or current presence of esophageal, gastric, and/or anorectal varices during endoscopy and/or on the previous or current presence of splenomegaly, collaterals, and/or abnormalities of portal blood flow during abdominal ultrasound with Doppler.

Healthy volunteers were recruited by local advertisement and considered eligible for inclusion in the control group when they had a normal medical history, physical examination, and liver tests [i.e. alanine transaminase (ALT) $<45 \mathrm{U} / \mathrm{L}$ and $\gamma$-glutamyl transpeptidase (GGT) $<55 \mathrm{U} / \mathrm{L}$ for men, and ALT $<35 \mathrm{U} / \mathrm{L}$ and GGT $<40 \mathrm{U} / \mathrm{L}$ for women], and were matched for age, sex and body mass index (BMI). Exclusion criteria included a history of gastrointestinal and/or liver diseases, major abdominal surgery interfering with gastrointestinal function, a history of alcohol abuse or excessive alcohol consumption (i.e. $>2$ alcoholic beverages per day or > 14 per week), use of medication that could affect intestinal permeability [e.g. non-steroidal anti-inflammatory drugs (NSAIDs) and proton pump inhibitors (PPIs)], or intestinal microbiota in the 2 months before the study.

\section{Study design}

This prospective case-control study consisted of two separate test days within a 4-week period. All subjects were requested to abstain from alcohol consumption on the day before their test day. After an overnight fast, subjects received an oral multisugar drink to assess intestinal permeability, consisting of $1 \mathrm{~g}$ sucrose (Van Gilse, 
Dinteloord, the Netherlands), $1 \mathrm{~g}$ lactulose (Centrafarm, Etten-Leur, the Netherlands), $0.5 \mathrm{~g}$ L-rhamnose (Danisco Sweeteners, Copenhagen, Denmark), $1 \mathrm{~g}$ erythritol (Danisco) and $1 \mathrm{~g}$ sucralose (Brenntag, Sittard, the Netherlands) dissolved in $150 \mathrm{ml}$ of tap water. Urine was collected in plastic containers for 24 hours in two separate fractions: $0-5 \mathrm{~h}$ and 5-24 $\mathrm{h}$. No eating or drinking (except tap water) was allowed during the first 5 hour urine collection. Before and after collection, weight of the containers was recorded, and aliquots of each fraction were stored at $-80^{\circ} \mathrm{C}$ until further analysis.

On the other test day, a gastroduodenoscopy and/or sigmoidoscopy were performed after an overnight fast but without prior bowel cleansing. Mucosal biopsies were obtained from standardized locations: the second segment of the duodenum and the sigmoid approximately $20 \mathrm{~cm}$ from the anal sphincter, respectively. Biopsies for gene transcription and protein expression were snap-frozen in liquid nitrogen and stored at $-80^{\circ} \mathrm{C}$ until further analyses. Biopsies for histological evaluation of hematoxylin and eosin-stained sections by one experienced pathologist were fixed in $4 \%$ formaldehyde and embedded separately in paraffin.

\section{Assessment of intestinal permeability}

Intestinal permeability was assessed using a validated multi-sugar test. ${ }^{18,19}$ Urine sugar concentrations were determined by isocratic ion-exchange high-pressure liquid chromatography (Model PU-1980 pump; Jasco, Easton, MD) with mass spectrometry (Model LTQ XL; Thermo Fisher Scientific, Walthan, MA) as described by van Wijck et al. ${ }^{18}$ Sucrose in 0-5 $\mathrm{h}$ urine, lactulose/rhamnose (L/R) ratio in 0-5 $\mathrm{h}$ urine and sucralose/erythritol (S/E) ratio in 5-24 h urine were used as indicators for gastroduodenal, small and large intestinal permeability, respectively.

\section{Quantitative Real-Time Polymerase Chain Reaction (qRT-PCR)}

Gene transcription of TJ and associated proteins, i.e. occludin, claudin-3, claudin-4, ZO1 and myosin light-chain kinase (MLCK), in mucosal biopsies was determined by qRTPCR. Total RNA was isolated from the frozen biopsies using TRIzol reagent (Invitrogen, Carlsbad, USA). RNA was purified with the RNeasy Mini Kit (Qiagen, Venlo, the Netherlands). The concentration of the purified RNA was measured with the NanoDrop. Finally, $500 \mathrm{ng}$ of total RNA was used as a template for the cDNA reaction, which was synthesized using the iScript cDNA Synthesis Kit (Bio-Rad, Veenendaal, the Netherlands). The cDNA was diluted to a concentration of $4 \mathrm{ng} / \mu \mathrm{L}$. Each reaction contained $5 \mu \mathrm{l}$ cDNA template solution, $12.5 \mu \mathrm{l}$ iQ SYBR Green Supermix (Bio-Rad), $1 \mu \mathrm{l}$ forward and reverse primers $(10 \mu \mathrm{M})$ and $5.5 \mu$ sterile water. Primer sequences have been listed in Table 3.1. Housekeeping genes included were 18S rRNA and glyceraldehyde-3-phosphate dehydrogenase (GAPDH). Reactions were run on the My IQ Single Color RT-PCR Detection System (Bio-Rad). PCR conditions used were $3 \mathrm{~min}$ at 
$95^{\circ} \mathrm{C}$, followed by 40 amplification cycles of $10 \mathrm{~s}$ at $95^{\circ} \mathrm{C}$ and $45 \mathrm{~s}$ at $60^{\circ} \mathrm{C}$. Data were presented as normalized expression ratios.

\begin{tabular}{|c|c|c|c|}
\hline & Sequence ID & Forward primer & Reverse primer \\
\hline 18S rRNA & M10098 & 5'-GTAACCCGTTGAACCCCATT-3' & 5'-CCATCCAATCGGTAGTAGCG-3' \\
\hline GAPDH & NM_002046 & 5'-TGCACCACCAACTGCTTAGC-3' & 5'-GGCATGGACTGTGGTCATGAG-3' \\
\hline Occludin & NM_002538.2 & 5'-TCAGGGAATATCCACCTATCACTTCAG-3' & 5'-CATCAGCAGCAGCCATGTACTCTTCAC-3' \\
\hline Claudin-3 & NM_001306.3 & 5'-TTCATCGGCAGCAACATCATC-3' & 5'-CGCCTGAAGGTCCTGTGG-3' \\
\hline Claudin-4 & NM_001305.3 & 5'-ACAGACAAGCCTTACTCC- $3^{\prime}$ & 5'-GGAAGAACAAAGCAGAG-3' \\
\hline ZO-1 & NM_003257.3 & 5'-AGGGGCAGTGGTGGTTTTCTGTTCTTTC-3' & 5'-GCAGAGGTCAAAGTTCAAGGCTCAAGAGG-3' \\
\hline MLCK & NM_053025.3 & 5-GCCTGACCACGAATATAAGTT-3' & 5'-GCTCCTTCTCATCATCATCTG-3' \\
\hline
\end{tabular}

$18 \mathrm{~S}$ rRNA, $18 \mathrm{~S}$ ribosomal RNA; GAPDH, glyceraldehyde-3-phosphate dehydrogenase; ZO-1, zonula occludens-1; MLCK, myosin light chain kinase.

\section{Enzyme-linked Immunosorbent Assay (ELISA)}

Protein levels of occludin and claudin-4 were determined in mucosal biopsies of duodenum and sigmoid. Frozen biopsies were ground with a mortar and pastel cooled in liquid nitrogen and resuspended in $100 \mu \mathrm{l}$ ice-cold PBS containing $10 \mu \mathrm{l} / \mathrm{mL}$ Protease Inhibitor Cocktail (Sigma-Aldrich, Zwijndrecht, the Netherlands). After centrifugation for $20 \mathrm{~min}\left(10,000\right.$ revolution/min, $\left.4^{\circ} \mathrm{C}\right)$, the supernatant was stored at $-80^{\circ} \mathrm{C}$ until further analysis. Total protein content in the supernatant was quantified using a BCA Protein Assay Kit (Pierce, Rockford IL). Occludin and claudin-4 in supernatant were determined by ELISA. In short, 96-well plates were coated overnight $\left(4^{\circ} \mathrm{C}\right)$ with $50 \mu \mathrm{l}$ tissue supernatant (1:10 dilution in PBS containing $10 \mu \mathrm{g}$ protein) or negative control (PBS). Thereafter, $50 \mu$ l blocking agent (3\% wt/vol BSA in PBS) was added for $1 \mathrm{~h}$ at $37^{\circ} \mathrm{C}$. The plate was washed, and then the wells were incubated with primary antibodies, polyclonal rabbit anti-occludin, or monoclonal mouse anti-claudin-4 (1:100 dilution in PBS containing 3\% BSA) (Invitrogen, Bleiswijk, the Netherlands) for $1 \mathrm{~h}$ at room temperature. The plates were washed, followed by the addition of secondary antibodies, horseradish-peroxidase (HRP)-conjugated anti-rabbit IgG (RayBiotech, Heerhugowaard, the Netherlands) and HRP-conjugated anti-mouse IgG (Dako, Heverlee, Belgium) (1:1.000 dilution in Assay Diluent, RayBiotech), and incubated for $2 \mathrm{~h}$ at room temperature. After being washed, TMB One-Step Substrate Reagent (RayBiotech) was added and incubated for $30 \mathrm{~min}$ in the dark to allow color development. The reaction was stopped by the addition of Stop Solution (RayBiotech), and absorbance of the color was measured at $450 \mathrm{~nm}$ by the Synergy HT MultiDetection Microplate Reader (Bio-Tek, Abcoude, the Netherlands). Data were expressed as optical density (OD) read at $450 \mathrm{~nm}$. 


\section{Power calculation and statistical analysis}

Primary outcome parameter of the study was small intestinal permeability. The number of subjects was determined using PS Power and Sample Size Calculations, based on previous studies in patients with compensated and decompensated liver cirrhosis $^{20}$ and in healthy subjects using indomethacin as stressor to increase small intestinal permeability. ${ }^{21}$ For the present study, 25 subjects were required per group to achieve an $80 \%$ power for detection of a statistically significant difference between both groups $(P<0.05)$ with $\alpha=0.05, \delta=0.017$ and $\sigma=0.021$.

Statistical analyses were performed using SPSS version 20.0. Data were tested for normality by using the Kolmogorov-Smirnoff test. Subsequently, continuous variables were presented as median (range) and compared between groups using the MannWhitney $U$-test for non-parametric data. Dichotomous variables were compared with the $\chi^{2}$ test. A $P$ value $<0.05$ was considered statistically significant using a two-tailed test.

\section{Results}

\section{Patients}

Twenty-six patients with compensated cirrhosis and 27 controls were included. Characteristics of subjects are given in Table 3.2. No significant differences with regard to age, sex or BMI were observed between patients with cirrhosis and controls. Serum ALT and GGT levels were significantly higher in patients with cirrhosis compared to controls $(P=0.025$ and $P<0.001$, respectively). Cause of liver cirrhosis was alcoholrelated in 11 patients, autoimmune-related in 6 patients, cryptogenic in 5 patients, chronic viral infection in 1 patient, hereditary hemochromatosis in 1 patient, and multifactorial in 2 patients. The majority of patients with alcohol-related cirrhosis $(n=9)$ was completely abstinent. Only two patients consumed alcohol on a regular basis but not on the day before the test day. Signs of portal hypertension were present in 24 of the 26 patients. Furthermore, 17 and 9 patients were classified as Child-Pugh class $A$ and $B$, respectively.

Drug therapy as part of the standard medical care was given to all patients, including, among others PPIs $(n=15)$, beta-blockers $(n=12)$, ursodeoxycholic acid $(n=11)$, diuretics $(n=7)$, lactulose $(n=6)$, glucocorticosteroids/immunosuppressives $(n=4)$, antibiotic prophylaxis $(n=1)$, anti-diarrheal agents $(n=1)$, and NSAIDs $(n=1)$. 
Table $3.2 \quad$ Characteristics of subjects.

\begin{tabular}{lccc}
\hline & $\begin{array}{c}\text { Patients with Cirrhosis } \\
(\mathrm{n}=26)\end{array}$ & $\begin{array}{c}\text { Controls } \\
(\mathrm{n}=27)\end{array}$ & $P$ value \\
\hline Age (years) & $61(18-72)$ & $60(19-78)$ & 0.539 \\
Sex (M / F) & $17 / 9$ & $17 / 10$ & 0.854 \\
BMI (kg/m²) & $25.8(18.8-39.0)$ & $26.0(18.1-32.1)$ & 0.689 \\
ALT (U/L) & $28.0(9.0-130.0)$ & $22.0(10.0-32.0)$ & 0.025 \\
GGT (U/L) & $51.0(17.0-259.0)$ & $22.0(6.0-50.0)$ & $<0.001$ \\
Child Pugh class (A/B/C) & $17 / 9 / 0$ & - & - \\
Child-Pugh score & $5.0(5.0-9.0)$ & - & - \\
MELD-score & $8.0(6.0-15.0)$ & - & - \\
\hline
\end{tabular}

Continuous values are presented as medians (range). BMI, body mass index; ALT, alanine aminotransferase; GGT, $\gamma$-glutamyl transpeptidase; MELD, model for end stage liver disease.

\section{Gastroduodenal, small and large intestinal permeability}

Gastroduodenal permeability, reflected by the 0-5 h urinary sucrose excretion, was not different between patients with cirrhosis and controls [5.979 (0.547-24.880) vs. 6.265 (2.226-30.469) $\mu \mathrm{mol} ; P=0.881$; Figure $3.1 \mathrm{~A}]$. The $0-5 \mathrm{~h}$ urinary L/R ratio, indicating small intestinal permeability, was also similar in both groups $[0.023(0.010-0.115)$ vs. 0.023 (0.006-0.069); $P=0.455$; Figure 3.1B]. However, large intestinal permeability was significantly increased in patients with cirrhosis compared to controls, as reflected by the increased 5-24 $\mathrm{h}$ urinary S/E ratio $[0.019(0.008-0.051)$ vs. $0.015(0.008-0.034)$; $P=0.006$; Figure $3.1 \mathrm{C}]$. The $0-24 \mathrm{~h}$ urinary $\mathrm{S} / \mathrm{E}$ ratio, indicating whole intestinal permeability, was also significantly increased in patients with cirrhosis compared to controls [0.020 (0.010-0.037) vs. $0.016(0.008-0.028) ; P=0.003]$.

Subanalysis based on the etiology of cirrhosis revealed a significantly increased $0-5 \mathrm{~h}$ urinary L/R ratio in patients with alcohol-related cirrhosis $(n=11)$ versus non-alcoholrelated cirrhosis $(n=15)$ [0.050 (0.010-0.115) vs. 0.020 (0.011-0.033); $P=0.016$; Figure 3.2], whereas the $0-5 \mathrm{~h}$ urinary sucrose excretion, $5-24 \mathrm{~h}$ and $0-24 \mathrm{~h}$ urinary S/E ratio did not differ between these subgroups of patients (data not shown). The 0-5 h urinary L/R ratio was also significantly increased between the subgroup of patients with alcohol-related cirrhosis and the controls $[0.050(0.010-0.115)$ vs. $0.023(0.006-0.069)$; $P=0.009$; Figure 3.2]. Use of PPIs and NSAIDs, which have been reported to be associated with a decreased barrier function, ${ }^{22,23}$ did not affect the urinary excretion of sucrose, the L/R ratio nor the S/E ratio in the patient group (data not shown). 
A

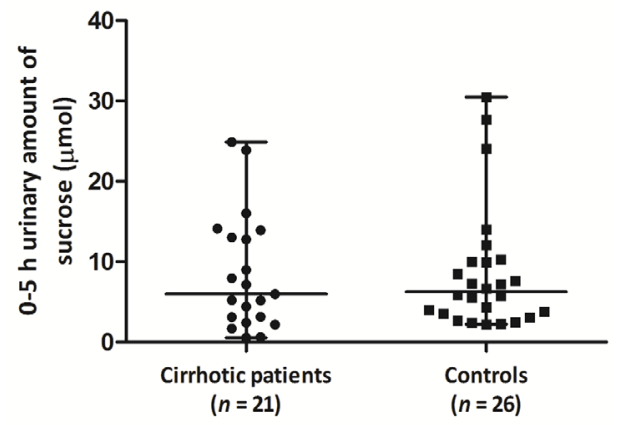

B

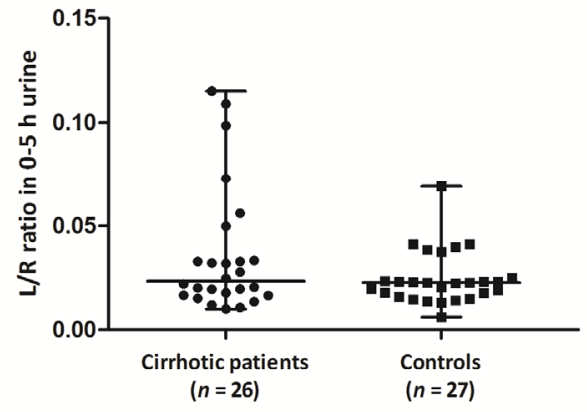

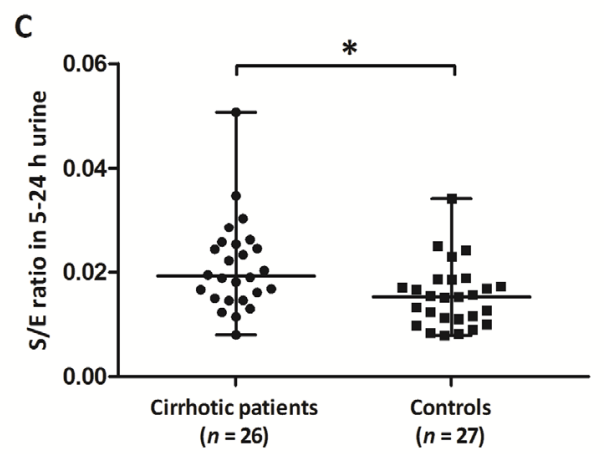

Figure 3.1 Intestinal permeability in patients with cirrhosis and controls. A: Gastroduodenal permeability (0-5 h urinary amount of sucrose). B: Small intestinal permeability [lactulose/rhamnose (L/R) ratio in 0-5 h urine]. C: Large intestinal permeability [sucralose/erythritol (S/E) ratio in 5-24 h urine]. Data are presented as scatter dot plots displaying median with range. $\left({ }^{*} P<0.01\right)$.

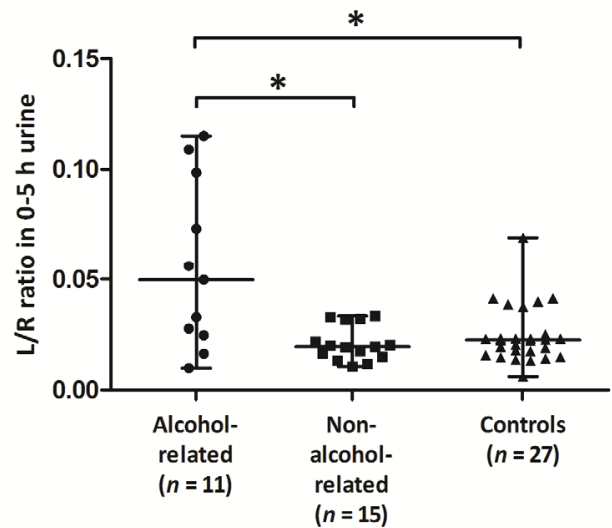

Figure 3.2 Small intestinal permeability (L/R) ratio in 0-5 h urine in patients with alcohol-related and nonalcohol-related cirrhosis and controls. Data are presented as scatter dot plots displaying median with range. $\left({ }^{*} P<0.05\right)$. 


\section{Gene transcription of TJ and associated proteins in duodenal and sigmoid biopsies}

Duodenal and sigmoid biopsies were obtained from 12 and 13 of the patients with cirrhosis, respectively, and from 22 controls. Gene transcription of claudin-3 was significantly downregulated in duodenal biopsies of patients with cirrhosis vs. controls [0.608 (0.363-1.397) vs. $1.400(0.722-3.911) ; P<0.001]$, whereas no significant differences were found for claudin-4, occludin, ZO-1, and MLCK between both groups (Table 3.3).

In sigmoid biopsies, gene transcription of claudin-4 was significantly downregulated in patients with cirrhosis compared to controls [0.866 (0.550-1.910) vs. 1.299 (0.192-5.310); $P=0.031$ ]. No significant differences in gene transcription were found for occludin, claudin-3, ZO-1, and MLCK (Table 3.3).

Table 3.3 Gene transcription of proteins associated with intestinal epithelial barrier function.

\begin{tabular}{lccc}
\hline & Patients with Cirrhosis & Controls & $P$ value \\
\hline Duodenal biopsies & & & \\
Occludin (11 vs. 22) & $0.824(0.587-1.055)$ & $1.050(0.372-4.674)$ & 0.158 \\
Claudin-3 (11 vs. 21) & $0.608(0.363-1.397)$ & $1.400(0.722-3.911)$ & $<0.001$ \\
Claudin-4 (11 vs. 22) & $0.835(0.483-1.852)$ & $1.229(0.419-4.933)$ & 0.061 \\
ZO-1 (10 vs. 22) & $0.781(0.418-1.188)$ & $0.473(0.266-2.461)$ & 0.143 \\
MLCK (11 vs. 22) & $0.802(0.200-1.087)$ & $0.708(0.335-2.896)$ & 0.593 \\
Sigmoid biopsies & & & \\
Occludin (13 vs. 21) & $0.975(0.540-1.910)$ & $0.571(0.133-2.744)$ & 0.058 \\
Claudin-3 (13 vs. 22) & $1.385(0.682-2.118)$ & $1.429(0.258-5.854)$ & 0.918 \\
Claudin-4 (13 vs. 22) & $0.866(0.550-1.910)$ & $1.299(0.192-5.310)$ & 0.031 \\
ZO-1 (13 vs. 21) & $0.822(0.275-2.155)$ & $0.703(0.157-2.886)$ & 0.280 \\
MLCK (13 vs. 22) & $1.305(0.720-1.740)$ & $1.189(0.139-2.736)$ & 0.838 \\
\hline
\end{tabular}

All data are normalized expression ratios, presented as medians (range).

\section{Protein levels of occludin and claudin-4 in duodenal and sigmoid biopsies}

Duodenal and sigmoid biopsies from 10 patients with cirrhosis and 22 controls were available for quantifying protein levels of occludin and claudin-4 by ELISA. Occludin levels were significantly increased in both duodenal and sigmoid biopsies of patients with cirrhosis vs. controls $[0.55(0.31-0.67)$ vs. $0.34(0.20-0.46), P<0.001$; and $(0.69$ (0.51-0.87) vs. 0.46 (0.28-0.63), $P<0.001$, respectively; Figure $3.3, A$ and B]. No significant differences were found for claudin-4 levels (Figure 3.3, C and D).

\section{Histology of mucosal biopsies}

Microscopically, only minor morphological changes were observed in duodenal biopsies of cirrhotic patients and controls that did not differ significantly between the groups, such as a (slightly) increased number of intraepithelial lymphocytes ( $n=2$ vs. $n=2$, respectively), mild edema of the lamina propria ( $n=2$ vs. $n=3$, respectively) and 
mild chronic inflammation ( $n=3$ vs. $n=2$, respectively). None of the subjects had villous atrophy or signs of acute inflammation. In sigmoid biopsies, only mild edema of the lamina propria was observed in both groups ( $n=4$ vs. $n=4$, respectively).
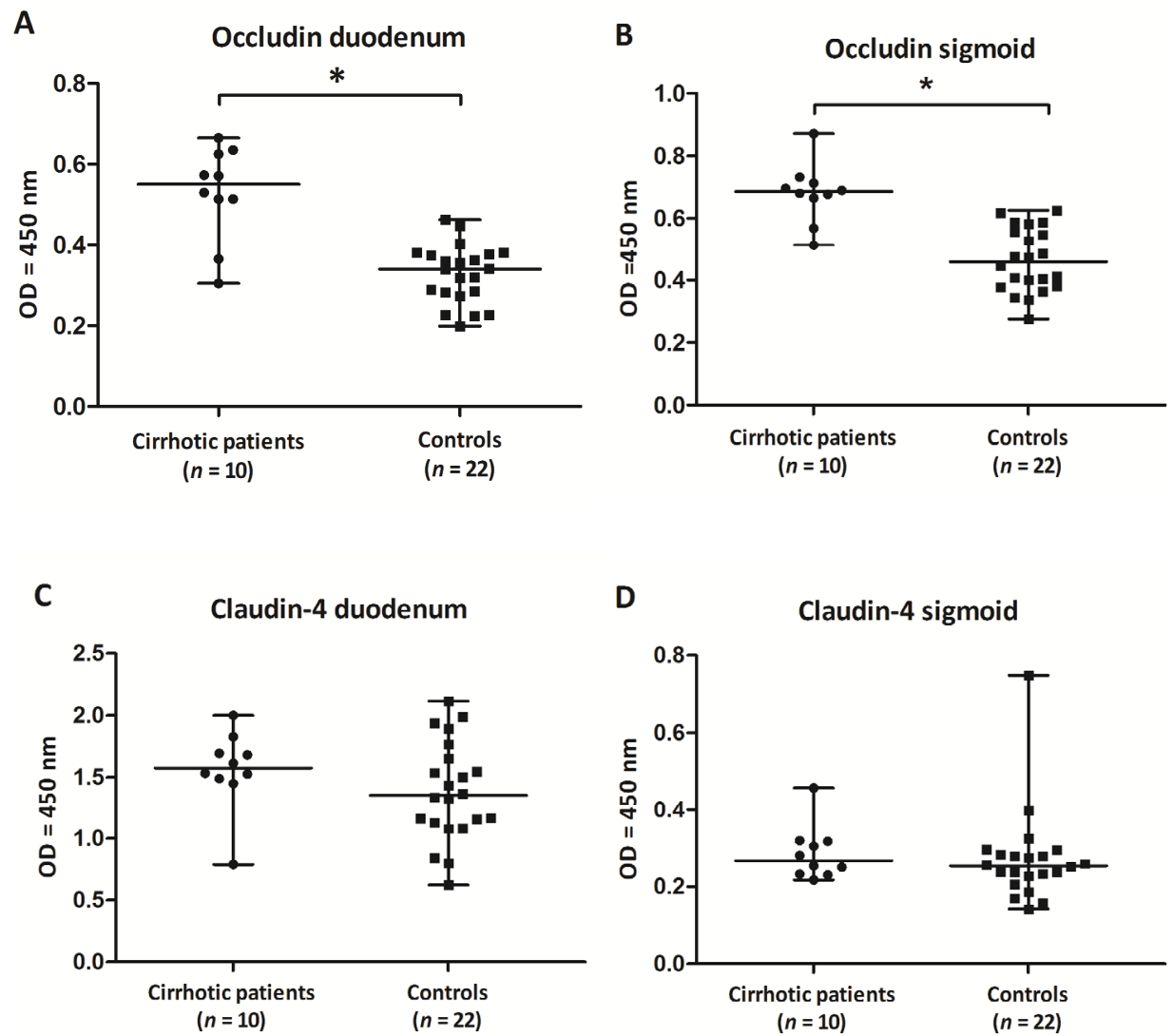

Figure 3.3 Protein levels of occludin and claudin-4 in mucosal biopsies of patients with cirrhosis and controls. Levels of occludin and claudin-4 were determined by ELISA. A: Protein levels of occludin in duodenal mucosa. B: Protein levels of occludin in sigmoid mucosa. C: Protein levels of claudin-4 in duodenal mucosa. D: Protein levels of claudin-4 in sigmoid mucosa. Data are expressed as optical density (OD) read at $450 \mathrm{~nm}$ and presented as scatter dot plots displaying median with range. $\left({ }^{*} P<0.001\right)$.

\section{Discussion}

In patients with compensated cirrhosis, large intestinal permeability but not gastroduodenal or small intestinal permeability was significantly increased. In addition, altered gene transcription was found for claudin- 3 in duodenal and claudin- 4 in 
sigmoid biopsies. At the protein level, occludin expression was significantly increased in both duodenal and sigmoid biopsies.

Several reports have shown that intestinal permeability is increased in patients with liver cirrhosis. ${ }^{7-12}$ Although, in most studies, mixed groups of patients with compensated and decompensated cirrhosis were included, we focused on patients with compensated cirrhosis and compared the results with age-, sex-, and BMImatched controls. Gastroduodenal and small intestinal permeability measured by the validated multi-sugar test were not affected in our patients. One should realize that several etiological factors leading to liver cirrhosis, such as alcohol and obesity (i.e. high fat diet) have been associated with a disturbed intestinal barrier function. ${ }^{24-27}$ Interestingly, in previous studies comparing subgroups of alcohol-related vs. nonalcohol-related cirrhosis, an increased small intestinal permeability in alcohol-related cirrhosis was observed, confirming a disruptive effect of alcohol on the intestinal epithelial barrier. ${ }^{25}$ Therefore, this may be a risk factor for bacterial translocation and could contribute to the progression towards decompensated cirrhosis. Although higher serum total IgA levels were observed in the subgroup of alcohol-related vs. nonalcohol-related cirrhosis, which might indicate an increased mucosal exposure to bacteria, ${ }^{28}$ we could not find an association with small intestinal permeability (data not shown). Both experimental and human studies have shown that acute (i.e. single, moderate or high intake) as well as chronic consumption of alcohol increases intestinal permeability. ${ }^{29-34}$ As in the present study, only two patients with alcohol-related cirrhosis still consumed alcohol regularly and were requested to abstain from alcohol the day before the test day; an acute effect of alcohol on intestinal permeability is not expected. It should be noted that sizes of subgroups are rather small, and our study was not powered for subgroup analysis of cirrhotic patients according to etiology.

Although we did not observe any change in the whole group of patients with compensated cirrhosis, we cannot exclude that gastroduodenal and small intestinal permeability are affected in patients with decompensated cirrhosis. Indeed, some studies have shown that increased intestinal permeability in patients with cirrhosis is associated with the presence of ascites. ${ }^{20,35,36}$ These changes in intestinal permeability may result from characteristics of patients with cirrhosis, such as alterations in the intestinal microbiota and/or immune system, systemic and local inflammation or oxidative stress and/or the presence of portal hypertension. ${ }^{4,37}$ As in the present study, almost all patients had signs of previous or current portal hypertension; overall we do not find an increased small intestinal permeability, and we do not expect portal hypertension to be a major risk factor in our population. This may be in part related to the fact that the degree of portal hypertension in this population does not exceed a certain limit, ${ }^{8}$ such as in decompensated cirrhotic patients in whom clinically evident complications resulting from portal hypertension (i.e. ascites, variceal hemorrhage and/or hepatic encephalopathy) are present.

Remarkably, we observed a significant increase in large intestinal permeability in patients with compensated cirrhosis measured by the S/E ratio in 5-24 $\mathrm{h}$ urine 
samples. Data on large intestinal permeability are scarce. Our findings confirm those of a recent study reporting an increased 5 to $26 \mathrm{~h}$ urinary excretion of sucralose in patients with cirrhosis. ${ }^{17}$ By combining two test markers different in molecular size and transport mechanism, we were able to correct for potential confounders, such as gastrointestinal transit and renal function. ${ }^{38}$

The increase in large intestinal permeability observed may be related to alterations in microbial composition by causing elevated endotoxin levels ${ }^{13,39}$ or by bacteria directly altering expression of TJ proteins. ${ }^{40}$ Changes in both fecal and sigmoid microbiota profiles have been reported in patients with cirrhosis compared to healthy controls. $^{15,41,42}$ Furthermore, as high numbers and diversity of bacteria are present in the large intestine, an increased permeability may enhance the risk of bacterial translocation. However, reliable analyses of (site-specific) bacterial translocation in humans require invasive techniques for sampling.

To support the functional permeability changes found by the multi-sugar test, we evaluated the expression of TJ proteins in both duodenal and sigmoid biopsies. We did not observe significant alterations at the TJ gene level apart from a downregulation of gene transcription of claudin- 3 in the duodenal mucosa and claudin- 4 in the sigmoid mucosa of patients with cirrhosis. Protein levels of occludin (but not of claudin-4) were significantly higher in duodenal and sigmoid mucosa of patients with cirrhosis compared to controls. After correction for multiple testing, gene transcription of claudin-4 was no longer significant.

The limited alterations on the gene and protein level are not clearly in line with the observed functional permeability changes. Inconsistent results are also reported by others showing altered and normal expression of TJ proteins in patients with cirrhosis with barrier dysfunction, ${ }^{13,14}$ indicating that the relation between functional and structural changes of the intestinal epithelial barrier in these patients needs further clarification. In addition to gene transcription, it has to be taken into account that e.g. posttranslational protein breakdown and/or modifications can contribute to "functional" protein levels.

A strength of our study is that we investigated the epithelial barrier of both the small and large intestine and combined functional analyses using the multi-sugar test with TJ analyses in biopsies focusing on patients with compensated cirrhosis.

Some potential shortcomings should also be taken into account. The multi-sugar test provides accurate, site-specific information on intestinal permeability and uses low sugar doses, ${ }^{19}$ but the $24 \mathrm{~h}$ urine collection can be a practical limitation for routine use. Second, expression of TJ proteins could only be evaluated in a subgroup of patients with cirrhosis. Although we acknowledge that translocation of bacteria and their products can occur via both transcellular and paracellular pathways and may be facilitated by changes in immune defense, ${ }^{4}$ the observed increase in large intestinal permeability may enable bacterial translocation. In the near future, it will also be interesting to examine biopsies from the ileum and proximal large intestine and to perform microbial analyses. Finally, it has to be noted that we included a 
heterogeneous group of patients with regard to etiology. Although we could demonstrate that patients with alcohol-related cirrhosis had an increased small intestinal permeability, the small sizes of other subgroups precluded us from performing additional analyses to investigate whether these subgroups may also be more susceptible for an altered intestinal permeability. Furthermore, various factors, including malnutrition ${ }^{17}$ and certain drugs ${ }^{22,23}$ may affect intestinal permeability. However, malnutrition was not observed in our study population, and use of PPIs and NSAIDs did not alter permeability results.

In conclusion, in patients with stable compensated cirrhosis, gastroduodenal and small intestinal permeability were not altered, whereas the large intestinal permeability was increased. Therefore, it would be interesting for future studies to further address the involvement of the large intestine regarding permeability and microbiota composition in bacterial translocation and in progression towards decompensated cirrhosis. 


\section{References}

1. Wiest R, Garcia-Tsao G. Bacterial translocation (BT) in cirrhosis. Hepatology 2005;41:422-33.

2. Pinzone MR, Celesia BM, Di Rosa M, Cacopardo B, Nunnari G. Microbial translocation in chronic liver diseases. Int J Microbiol 2012;2012:694629.

3. Bellot $P$, Frances R, Such J. Pathological bacterial translocation in cirrhosis: pathophysiology, diagnosis and clinical implications. Liver Int 2013;33:31-9.

4. Wiest R, Lawson M, Geuking M. Pathological bacterial translocation in liver cirrhosis. J Hepatol 2014;60:197-209.

5. Turner JR. Intestinal mucosal barrier function in health and disease. Nat Rev Immunol 2009;9:799-809.

6. Van Itallie CM, Anderson JM. The molecular physiology of tight junction pores. Physiology (Bethesda) 2004;19:331-8.

7. Campillo B, Pernet P, Bories PN, Richardet JP, Devanlay M, Aussel C. Intestinal permeability in liver cirrhosis: relationship with severe septic complications. Eur J Gastroenterol Hepatol 1999;11:755-9.

8. Ersoz G, Aydin A, Erdem S, Yuksel D, Akarca U, Kumanlioglu K. Intestinal permeability in liver cirrhosis. Eur J Gastroenterol Hepatol 1999;11:409-12.

9. Choi Y, Jeon WK, Hwang SJ, et al. The role of the gut barrier function in the pathophysiology of viral liver cirrhosis. Hepatogastroenterology 2011;58:1244-7.

10. Benjamin J, Singla V, Arora I, Sood S, Joshi YK. Intestinal permeability and complications in liver cirrhosis: A prospective cohort study. Hepatol Res 2013;43:200-7.

11. Pascual S, Such J, Esteban A, et al. Intestinal permeability is increased in patients with advanced cirrhosis. Hepatogastroenterology 2003;50:1482-6.

12. Scarpellini E, Valenza V, Gabrielli M, et al. Intestinal Permeability in Cirrhotic Patients With and Without Spontaneous Bacterial Peritonitis: Is the Ring Closed[quest]. Am J Gastroenterol 2009;105:323-7.

13. Assimakopoulos SF, Tsamandas AC, Tsiaoussis GI, et al. Altered intestinal tight junctions' expression in patients with liver cirrhosis: a pathogenetic mechanism of intestinal hyperpermeability. Eur J Clin Invest 2012;42:439-46.

14. Du Plessis J, Vanheel H, Janssen CE, et al. Activated intestinal macrophages in patients with cirrhosis release NO and IL-6 that may disrupt intestinal barrier function. J Hepatol 2013;58:1125-32.

15. Bajaj JS, Hylemon PB, Ridlon JM, et al. Colonic mucosal microbiome differs from stool microbiome in cirrhosis and hepatic encephalopathy and is linked to cognition and inflammation. Am J Physiol Gastrointest Liver Physiol 2012;303:G675-85.

16. Elamin E, Jonkers D, Troost F, Dekker J, Masclee A. Tu1352 Moderate Dosage of Ethanol Increases Small and Large Intestinal Permeability in Healthy Volunteers. Gastroenterology 2012;142:S-809.

17. Norman K, Pirlich M, Schulzke JD, et al. Increased intestinal permeability in malnourished patients with liver cirrhosis. Eur J Clin Nutr 2012;66:1116-9.

18. van Wijck K, van Eijk HM, Buurman WA, Dejong $\mathrm{CH}$, Lenaerts $\mathrm{K}$. Novel analytical approach to a multisugar whole gut permeability assay. J Chromatogr B Analyt Technol Biomed Life Sci 2011;879:2794801.

19. van Wijck K, Verlinden TJ, van Eijk HM, et al. Novel multi-sugar assay for site-specific gastrointestinal permeability analysis: a randomized controlled crossover trial. Clin Nutr 2013;32:245-51.

20. Zuckerman MJ, Menzies IS, Ho H, et al. Assessment of Intestinal Permeability and Absorption in Cirrhotic Patients with Ascites Using Combined Sugar Probes. Dig Dis Sci 2004;49:621-6.

21. Troost FJ, Saris WH, Brummer RJ. Recombinant human lactoferrin ingestion attenuates indomethacininduced enteropathy in vivo in healthy volunteers. Eur J Clin Nutr 2003;57:1579-85.

22. Bjarnason I, Takeuchi K. Intestinal permeability in the pathogenesis of NSAID-induced enteropathy. J Gastroenterol 2009;44 Suppl 19:23-9.

23. Mullin JM, Valenzano MC, Whitby $M$, et al. Esomeprazole induces upper gastrointestinal tract transmucosal permeability increase. Aliment Pharmacol Ther 2008;28:1317-25.

24. Purohit V, Bode JC, Bode C, et al. Alcohol, intestinal bacterial growth, intestinal permeability to endotoxin, and medical consequences: summary of a symposium. Alcohol 2008;42:349-61.

25. Elamin EE, Masclee AA, Dekker J, Jonkers DM. Ethanol metabolism and its effects on the intestinal epithelial barrier. Nutr Rev 2013;71:483-99. 
26. Teixeira TF, Collado MC, Ferreira CL, Bressan J, Peluzio Mdo C. Potential mechanisms for the emerging link between obesity and increased intestinal permeability. Nutr Res 2012;32:637-47.

27. Moreira AP, Texeira TF, Ferreira AB, Peluzio Mdo C, Alfenas Rde C. Influence of a high-fat diet on gut microbiota, intestinal permeability and metabolic endotoxaemia. Br J Nutr 2012;108:801-9.

28. Colombel JF, Vaerman JP, Mesnard B, Dehennin JP, Dive C, Rambaud JC. Jejunal immunoglobulin secretion in alcoholic patients with and without cirrhosis. J Hepatol 1991;12:145-9.

29. Ma TY, Nguyen D, Bui V, Nguyen H, Hoa N. Ethanol modulation of intestinal epithelial tight junction barrier. Am J Physiol 1999;276:G965-74.

30. Banan A, Choudhary S, Zhang Y, Fields JZ, Keshavarzian A. Ethanol-induced barrier dysfunction and its prevention by growth factors in human intestinal monolayers: evidence for oxidative and cytoskeletal mechanisms. J Pharmacol Exp Ther 1999;291:1075-85.

31. Keshavarzian A, Choudhary S, Holmes EW, et al. Preventing gut leakiness by oats supplementation ameliorates alcohol-induced liver damage in rats. J Pharmacol Exp Ther 2001;299:442-8.

32. Keshavarzian A, Farhadi A, Forsyth $C B$, et al. Evidence that chronic alcohol exposure promotes intestinal oxidative stress, intestinal hyperpermeability and endotoxemia prior to development of alcoholic steatohepatitis in rats. J Hepatol 2009;50:538-47.

33. Robinson GM, Orrego H, Israel Y, Devenyi P, Kapur BM. Low-molecular-weight polyethylene glycol as a probe of gastrointestinal permeability after alcohol ingestion. Dig Dis Sci 1981;26:971-7.

34. Parlesak A, Schäfer C, Schütz T, Bode JC, Bode C. Increased intestinal permeability to macromolecules and endotoxemia in patients with chronic alcohol abuse in different stages of alcohol-induced liver disease. J Hepatol 2000;32:742-7.

35. Lee S, Son SC, Han MJ, et al. Increased intestinal macromolecular permeability and urine nitrite excretion associated with liver cirrhosis with ascites. World J Gastroenterol 2008;14:3884-90.

36. Kalaitzakis E, Johansson JE, Bjarnason I, Bjornsson E. Intestinal permeability in cirrhotic patients with and without ascites. Scand J Gastroenterol 2006;41:326-30.

37. Pijls KE, Jonkers DM, Elamin EE, Masclee AA, Koek GH. Intestinal epithelial barrier function in liver cirrhosis: an extensive review of the literature. Liver Int 2013;33:1457-69.

38. Bjarnason I, MacPherson A, Hollander D. Intestinal permeability: an overview. Gastroenterology 1995;108:1566-81.

39. Forsythe RM, Xu DZ, Lu Q, Deitch EA. Lipopolysaccharide-induced enterocyte-derived nitric oxide induces intestinal monolayer permeability in an autocrine fashion. Shock 2002;17:180-4.

40. Qin H, Zhang Z, Hang X, Jiang Y. L. plantarum prevents enteroinvasive Escherichia coli-induced tight junction proteins changes in intestinal epithelial cells. BMC Microbiol 2009;9:63.

41. Chen $\mathrm{Y}$, Yang $\mathrm{F}$, Lu H, et al. Characterization of fecal microbial communities in patients with liver cirrhosis. Hepatology 2011;54:562-72.

42. Bajaj JS, Ridlon JM, Hylemon PB, et al. Linkage of gut microbiome with cognition in hepatic encephalopathy. Am J Physiol Gastrointest Liver Physiol 2012;302:G168-75. 


\section{Chapter 4}

Is intestinal oxidative stress involved in patients with compensated liver cirrhosis?

K.E. Pijls

D.M.A.E. Jonkers

M. Elizalde

M.J. Drittij-Reijnders

G.R. Haenen

A. Bast

A.A.M. Masclee

G.H. Koek

Ann Hepatol 2016; 15:402-409 


\section{Abstract}

Liver cirrhosis is associated with intestinal epithelial barrier dysfunction, which may be affected by oxidative stress. Studies in cirrhotic rats provided evidence for intestinal oxidative stress, but studies in cirrhotic patients are scarce. We have shown intestinal barrier dysfunction in patients with compensated cirrhosis. The present study aimed to investigate whether oxidative stress occurs in the intestinal mucosa of compensated cirrhotic patients and may contribute to barrier dysfunction.

Oxidative stress was studied in duodenal and sigmoid biopsies from 15 cirrhotic patients and 22 controls by analyzing transcription of genes involved in glutathione and uric acid metabolism using quantitative real-time polymerase chain reaction. Protein levels of glutathione and glutathione disulphide were measured and the glutathione/glutathione disulphide ratio was calculated as marker of oxidative stress. In addition, intestinal myeloperoxidase and fecal calprotectin were determined.

Gene transcription of glutathione synthetase and glutathione reductase were significantly different in duodenal and sigmoid biopsies of cirrhotic patients vs. controls, but no alterations were found for other genes nor for glutathione, glutathione disulphide, glutathione/glutathione disulphide ratio or intestinal myeloperoxidase and fecal calprotectin concentrations.

This study did not find indications for oxidative stress and low-grade inflammation in the small and large intestine of stable compensated cirrhotic patients. Although these preliminary findings need further validation, we found intestinal oxidative stress not to be a major mechanism contributing to epithelial barrier dysfunction in patients with compensated cirrhosis. 


\section{Introduction}

Cirrhosis is the end stage of chronic liver diseases and is associated with a high morbidity and mortality. Increasing evidence indicates that the gastrointestinal (GI) tract plays a role in the development of liver cirrhosis and its complications. Cirrhosis has been associated with both structural and functional alterations in the GI tract, such as edema of lamina propria, ${ }^{1}$ distended intercellular spaces, ${ }^{2}$ impaired motility, ${ }^{3}$ changes in microbiota composition ${ }^{4}$ and dysfunction of the epithelial barrier. Several studies have shown an increased intestinal permeability in patients with liver cirrhosis. $^{5-7}$ This may facilitate translocation of bacteria and bacterial products such as endotoxin into the systemic circulation, ${ }^{8}$ and thereby contribute to the development of cirrhotic complications, such as spontaneous bacterial peritonitis. ${ }^{9}$ Epithelial barrier function is regulated by intercellular tight junctions (TJ) and adherens junctions (AJ). Recently, Assimakopoulos et al. ${ }^{10}$ and Du Plessis et al., ${ }^{11}$ found alterations in the expression of TJ proteins in duodenal biopsies of compensated and decompensated cirrhotic patients.

Oxidative stress is a potential mechanism underlying intestinal epithelial barrier dysfunction as it can induce direct epithelial cell damage and disrupt TJ and/or AJ function and structure. ${ }^{12-15}$ Possible factors causing oxidative damage in the intestine of patients with cirrhosis include ingested alcohol, ${ }^{16}$ changes in intestinal microbiota, ${ }^{4,17,18}$ intestinal inflammation ${ }^{11,19}$ and disturbed microcirculation of the intestinal mucosa secondary to portal hypertension. ${ }^{1,20,21}$ In addition, systemic inflammation and oxidative stress as well as oxidative stress inducers produced in the liver, may be transferred to the intestine via blood and bile. ${ }^{15,22}$ These factors together with a decreased antioxidant status in cirrhotic patients ${ }^{23}$ may promote intestinal oxidative damage.

Few studies in rats with carbon tetrachloride $\left(\mathrm{CCl}_{4}\right)$-induced cirrhosis have provided evidence for oxidative stress in both the small and large intestine, by e.g. increased xanthine oxidase (XO) activity, increased malondialdehyde (MDA) levels, and alterations in antioxidant status, such as low levels of reduced glutathione (GSH) and high levels of glutathione disulphide (GSSG). ${ }^{13,24-26}$ So far, one recently published study has assessed mucosal proliferation, apoptosis and oxidative stress in duodenal biopsies of patients with cirrhosis and found a significantly increased intestinal lipid peroxidation (i.e. lipid hydroperoxides) as well as increased plasma endotoxin concentrations. $^{27}$

In a previous study we have investigated the intestinal epithelial barrier function in patients with compensated liver cirrhosis in order to find out whether an increased intestinal permeability may be a risk factor for the progression towards decompensated cirrhosis and observed an increased small intestinal permeability in a subgroup of patients with alcohol-related cirrhosis and an increased large intestinal permeability in the whole group of compensated cirrhotic patients. ${ }^{28}$ 
We hypothesized that intestinal oxidative stress is involved in the epithelial barrier dysfunction of patients with compensated liver cirrhosis. Aim of the present study was therefore to investigate the occurrence of oxidative stress not only in the mucosa of the small, but also of the large intestine in patients with compensated cirrhosis and to compare data with those obtained in healthy controls.

\section{Materials and methods}

\section{Patients and study design}

A subgroup of compensated cirrhotic patients (i.e. without clinically evident complications, including ascites, variceal hemorrhage, hepatic encephalopathy and/or jaundice) and a control group of healthy volunteers participating in a prior prospective case-control study on intestinal permeability were available for analyses of intestinal oxidative stress. The design and clinical details of the study have been described elsewhere. ${ }^{28}$ Briefly, 15 cirrhotic patients and 22 controls underwent a gastroduodenoscopy and/or sigmoidoscopy after an overnight fast but without prior bowel cleansing. Mucosal biopsies were obtained from standardized locations: the second segment of the duodenum and the sigmoid approximately $20 \mathrm{~cm}$ from the anal sphincter, respectively. Biopsies for gene transcription and protein expression were snap-frozen in liquid nitrogen and stored at $-80^{\circ} \mathrm{C}$ until further analyses. Biopsies for histological evaluation of haematoxylin and eosin-stained sections by one experienced pathologist, were fixed in $4 \%$ formaldehyde and embedded in paraffin.

In addition, subjects collected a fresh fecal sample in a sterile container. Aliquots were stored within 12 hours after defecation at $-80^{\circ} \mathrm{C}$ until further analysis.

The study has been approved by the Medical Ethics Committee of Maastricht University Medical Center (MUMC), conducted according to the revised version of the Declaration of Helsinki (October 2008, Seoul) and registered at the US National Library of Medicine (http://www.clinicaltrials.gov, NTC01081236). All subjects (patients and healthy volunteers) gave their written informed consent before participation.

\section{Transcription of oxidative stress-related genes}

Transcription of genes involved in glutathione (GSH) and uric acid metabolism, i.e. glutamate-cysteine ligase, catalytic subunit (GCLC), glutamate-cysteine ligase, modifier subunit (GCLM), glutathione synthetase (GS), glutathione peroxidases (GPX1, GPX2 and GPX3), glutathione reductase (GR), and xanthine dehydrogenase $(X D H)$, in mucosal biopsies were determined by qRT-PCR. Total RNA was isolated from the frozen biopsies using TRIzol reagent (Invitrogen, Carlsbad, USA) and purified with the RNeasy Mini Kit (Qiagen, Venlo, the Netherlands). The concentration of purified RNA was measured with the NanoDrop. Finally, $500 \mathrm{ng}$ total RNA was used as a template for the cDNA 
reaction, which was synthesized using the iScript cDNA Synthesis Kit (Bio-Rad, Veenendaal, the Netherlands). The cDNA was diluted to a concentration of $4 \mathrm{ng} / \mu \mathrm{L}$. Each reaction contained $5 \mu \mathrm{L}$ cDNA template solution, $12.5 \mu \mathrm{L}$ iQ SYBR Green Supermix (Bio-Rad), $1 \mu \mathrm{L}$ forward and reverse primers $(10 \mu \mathrm{M})$ and $5.5 \mu \mathrm{L}$ sterile water. Primer sequences have been listed in Table 4.1. Housekeeping genes included were $18 \mathrm{~S}$ rRNA and glyceraldehyde-3-phosphate dehydrogenase (GAPDH). Reactions were run on the My IQ Single Color RT-PCR Detection System (Bio-Rad). PCR conditions used were 3 min at $95^{\circ} \mathrm{C}$, followed by 40 amplification cycles of $10 \mathrm{~s}$ at $95^{\circ} \mathrm{C}$ and $45 \mathrm{~s}$ at $60^{\circ} \mathrm{C}$. Data were expressed as normalized expression ratios.

Table 4.1 Primer sequences of housekeeping genes and genes involved in glutathione and uric acid metabolism.

\begin{tabular}{llll}
\hline & Sequence ID & Forward primer & Reverse primer \\
\hline 18S rRNA & M10098 & 5'-GTAACCCGTTGAACCCCATT-3' & 5'-CCATCCAATCGGTAGTAGCG-3' \\
GAPDH & NM_002046 & 5'-TGCACCACCAACTGCTTAGC-3' & 5'-GGCATGGACTGTGGTCATGAG-3' \\
GCLC & NM_001498 & 5'-TGGAAGTGGATGTGGACACC-3' & 5'-GTCTTGCTTGTAGTCAGGATGG-3' \\
GCLM & NM_002061 & 5'-GGCACAGGTAAAACCAAATAGTAAC-3' & 5'-CAAATTGTTTAGCAAATGCAGTCA-3' \\
GS & NM_000178 & 5'-AAGACACTCGTGATGAACAAGC-3' & 5'-GGAGAGGAATGACAAATACAGAGG-3' \\
GPX1 & NM_000581 & 5'-CCGACCCCAAGCTCATCACC-3' & 5'-GATGTCAGGCTCGATGTCAATGG-3' \\
GPX2 & NM_002083 & 5'-ATCCTGAACAGTCTCAAGTATG-3' & 5'-TGGGTCATCATAAGGGTAGG-3' \\
GPX3 & NM_002084 & 5'-ACATGCCTACAGGTATGCGTGATTG-3' & 5'-TGGAGTGGAGAACTGGAGAGAAAGG-3' \\
GR & NM_000637 & 5'-CAGGGACTTGGGTGTGATGAAATGC-3' & 5'-GAGGTAGGGTGAATGGCGACTGTG-3' \\
XDH & NM_000379.3 & 5'-CCTCTTCCTGGCTGCTTCTATCTTC-3' & 5'-TGACACACAGGGTGGTGAACTTG-3' \\
\hline
\end{tabular}

18S rRNA, 18S ribosomal RNA; GAPDH, glyceraldehyde-3-phosphate dehydrogenase; GCLC, glutamate-cysteine ligase, catalytic subunit; GCLM, glutamate-cysteine ligase, modifier subunit; GS, glutathione synthetase; GPX, glutathione peroxidase; GR, glutathione reductase; $\mathrm{XDH}$, xanthine dehydrogenase.

\section{GSH/GSSG ratio and myeloperoxidase in mucosal biopsies}

Frozen biopsies for oxidative stress analyses were ground with a mortar and pastel cooled in liquid nitrogen, and resuspended in $220 \mu \mathrm{L}$ ice-cold milliQ. Two hundred $\mu \mathrm{L}$ from this suspension was added to $20 \mu \mathrm{L}$ of an acidic buffer (13\% 5-Sulfosalicilic acid, $100 \mathrm{mmol} / \mathrm{L} \mathrm{HCl}$ in PBS). After centrifugation, the supernatant was used to determine the concentrations of GSH and GSSG using the method described by Julicher et al. ${ }^{29}$

Frozen biopsies for myeloperoxidase (MPO) analyses were ground with a mortar and pastel cooled in liquid nitrogen, and resuspended in $100 \mu \mathrm{L}$ ice-cold PBS containing 10 $\mu \mathrm{L} / \mathrm{mL}$ Protease Inhibitor Cocktail (Sigma-Aldrich, Zwijndrecht, the Netherlands). After centrifugation for $20 \mathrm{~min}\left(10.000 \mathrm{rpm}, 4^{\circ} \mathrm{C}\right)$, the supernatant was stored at $-80^{\circ} \mathrm{C}$ until further analysis. MPO in supernatant was determined using an ELISA Kit (HBT, Uden, the Netherlands) according to the manufacturer's instructions. Total protein content in the supernatants was quantified using the BCA Protein Assay Kit (Pierce ${ }^{\mathrm{TM}}$, Rockford IL, USA).

GSH and GSSG were expressed as nmol/mg protein in biopsies and the GSH/GSSG ratio was calculated as a marker of oxidative stress. MPO was expressed as $\mu \mathrm{g} / \mathrm{g}$ protein in biopsies. 


\section{Fecal calprotectin}

Approximately $100 \mathrm{mg}$ of wet weight feces was diluted 50 times in extraction buffer $\left(0.1 \mathrm{~mol} / \mathrm{L}\right.$ Tris, $0.15 \mathrm{~mol} / \mathrm{L} \mathrm{NaCl}, 1 \mathrm{~mol} / \mathrm{L}$ Urea, $10 \mathrm{mmol} / \mathrm{L} \mathrm{CaCl} 2 \cdot 2 \mathrm{H}_{2} \mathrm{O}, 0.1 \mathrm{~mol} / \mathrm{L} \mathrm{Citric}$ acid, $5 \mathrm{~g} / \mathrm{L} \mathrm{BSA}, \mathrm{pH} ; 8.0) .^{30}$ Samples were shaken for $30 \mathrm{~min}$ and subsequently centrifuged for $20 \mathrm{~min}\left(10.000 \mathrm{rpm}, 4^{\circ} \mathrm{C}\right)$. Supernatants were used for analysis of calprotectin using a standard ELISA Kit (HBT) according to the manufacturer's instructions. Data were expressed as $\mu \mathrm{g} / \mathrm{g}$ feces.

\section{Statistical analysis}

Statistical analyses were performed using SPSS version 20.0. Data were tested for normality by the Kolmogorov-Smirnoff test. Subsequently, continuous variables were presented as median (range) and compared between groups using the Mann-Whitney $U$-test for non-parametric data. Dichotomous variables were compared with the $\chi^{2}$ test. A $P<0.05$ was considered statistically significant using a two-tailed test.

\section{Results}

\section{Patients}

Duodenal and/or sigmoid biopsies were obtained from 15 compensated cirrhotic patients (i.e. 12 duodenal and 13 sigmoid biopsies) and from 22 healthy controls (i.e. 22 duodenal and 22 sigmoid biopsies). Characteristics of subjects are given in Table 4.2. No significant differences with regard to age, sex or BMI were observed between cirrhotic patients and controls. Serum alanine transaminase (ALT) and $\gamma$-glutamyl transpeptidase (GGT) levels were significantly higher in cirrhotic patients compared to controls ( $P=0.004$ and $P<0.001$, respectively). Furthermore, 11 and 4 patients were classified as Child-Pugh class $A$ and $B$, respectively. None of the patients had clinically evident complications, i.e. ascites, variceal hemorrhage, hepatic encephalopathy and/or jaundice. Cause of liver cirrhosis was alcohol-related in 4 patients, autoimmune-related in 3 patients, cryptogenic in 4 patients, chronic viral infection in 1 patient, hereditary hemochromatosis in 1 patient and multifactorial in 2 patients.

Drug therapy as part of the standard medical care was given to all patients, including among others glucocorticosteroids/immunosuppressives $(n=2)$, non-steroidal antiinflammatory drugs (NSAIDs) $(n=1)$ and colchicine $(n=1)$.

\section{Transcription of oxidative stress-related genes}

Gene transcription of GR was significantly upregulated in duodenal biopsies of cirrhotic patients vs. controls [2.11 (0.39-4.44) vs. $1.28(0.51-2.48) ; P=0.047]$, whereas no 
significant differences were found for GCLC, GCLM, GS, GPX1, GPX2, GPX3 and XDH between both groups (Table 4.3).

In sigmoid biopsies, gene transcription of both GS and GR was significantly downregulated in cirrhotic patients compared to controls [0.68 (0.15-2.45) vs. 1.24 (0.43-3.64); $P=0.013$ and 0.90 (0.18-2.99) vs. 2.07 (0.48-10.88); $P=0.022$ ]. No significant differences in gene transcription were found for GCLC, GCLM, GPX1, GPX2, GPX3 and XDH (Table 4.3).

Table $4.2 \quad$ Characteristics of subjects.

\begin{tabular}{lccc}
\hline & $\begin{array}{c}\text { Patients with Cirrhosis } \\
(\mathrm{n}=15)\end{array}$ & $\begin{array}{c}\text { Controls } \\
(\mathrm{n}=22)\end{array}$ & $P$ value \\
\hline Age (years) & $63(18-72)$ & $47(19-78)$ & 0.377 \\
Sex $(\mathrm{M} \mathrm{/} \mathrm{F)}$ & $10 / 5$ & $12 / 10$ & 0.461 \\
$\mathrm{BMI}\left(\mathrm{kg} / \mathrm{m}^{2}\right)$ & $25.9(19.6-39.0)$ & $25.0(18.1-29.8)$ & 0.467 \\
$\mathrm{ALT}(\mathrm{U} / \mathrm{L})^{*}$ & $30.0(9.0-130.0)$ & $19.5(10.0-32.0)$ & 0.004 \\
GGT (U/L)* & $47.0(17.0-194.0)$ & $19.5(6.0-40.0)$ & $<0.001$ \\
Child Pugh class (A/B/C) & $11 / 4 / 0$ & - & - \\
Child-Pugh score & $5.0(5.0-9.0)$ & - & - \\
MELD-score & $8.0(6.0-15.0)$ & - & - \\
\hline
\end{tabular}

Continuous values are presented as medians (range). *Significant difference, $P<0.05$.

$\mathrm{M}$, male; $\mathrm{F}$, female; BMI, body mass index; ALT, alanine transaminase; U/L, Units per liter; GGT, $\mathrm{Y}$-glutamyl transpeptidase; MELD-score, Model for End Stage Liver Disease-score.

Table 4.3 Transcription of genes involved in glutathione and uric acid metabolism

\begin{tabular}{lcll}
\hline & Patients with Cirrhosis & \multicolumn{1}{c}{ Controls } & $P$ value \\
\hline Duodenal biopsies & & & \\
GCLC (12 vs. 22) & $1.17(0.28-5.57)$ & $0.60(0.13-1.85)$ & 0.140 \\
GCLM (12 vs. 22) & $1.40(0.21-5.57)$ & $0.91(0.24-2.73)$ & 0.207 \\
GS (12 vs. 22) & $0.99(0.11-3.08)$ & $0.79(0.18-1.66)$ & 0.387 \\
GPX1 (12 vs. 22) & $0.95(0.25-3.28)$ & $1.14(0.25-2.85)$ & 0.449 \\
GPX2 (12 vs. 22) & $0.85(0.18-5.57)$ & $0.67(0.18-1.91)$ & 0.207 \\
GPX3 (12 vs. 22) & $1.91(0.14-4.83)$ & $1.00(0.28-3.38)$ & 0.061 \\
GR (12 vs. 22)* & $2.11(0.39-4.44)$ & $1.28(0.51-2.48)$ & 0.047 \\
XDH (12 vs. 22) & $1.14(0.10-3.43)$ & $0.92(0.21-1.71)$ & 0.564 \\
Sigmoid biopsies† & & & \\
GCLC (13 vs. 19) & $0.97(0.34-4.00)$ & $1.68(0.41-4.38)$ & 0.058 \\
GCLM (13 vs. 19) & $0.68(0.20-3.09)$ & $0.67(0.11-13.35)$ & 0.774 \\
GS (13 vs. 19)* & $0.68(0.15-2.45)$ & $1.24(0.43-3.64)$ & 0.013 \\
GPX1 (13 vs. 19) & $1.19(0.24-7.33)$ & $1.54(0.74-3.74)$ & 0.291 \\
GPX2 (13 vs. 19) & $0.56(0.11-2.28)$ & $0.96(0.22-2.70)$ & 0.173 \\
GPX3 (13 vs. 18) & $1.38(0.13-5.47)$ & $0.81(0.15-5.04)$ & 0.447 \\
GR (13 vs. 19)* & $0.90(0.18-2.99)$ & $2.07(0.48-10.88)$ & 0.022 \\
XDH (13 vs. 18) & $1.82(0.13-7.64)$ & $0.64(0.09-9.08)$ & 0.378 \\
\hline
\end{tabular}

All data are normalized expression ratios, presented as medians (range). *Significant difference, $P<0.05$.

tGood quality RNA could only be obtained from 19 of 22 sigmoid biopsies.

GCLC, glutamate-cysteine ligase, catalytic subunit; GCLM, glutamate-cysteine ligase, modifier subunit; GS, glutathione synthetase; GPX, glutathione peroxidase; GR, glutathione reductase; XDH, xanthine dehydrogenase. 


\section{GSH/GSSG ratio and MPO in mucosal biopsies}

Concentrations of GSH and GSSG, and the GSH/GSSG ratio did not differ significantly between cirrhotic patients and controls in duodenal nor in sigmoid biopsies (Table 4.4 and Figure 4.1A and B).

To further support the oxidative stress data, local intestinal inflammation was assessed by analysis of MPO levels in the mucosal biopsies. ${ }^{31}$ The concentrations of MPO were also not significantly different between both groups in duodenal [17.57 (7.51-30.21) $\mu \mathrm{g} / \mathrm{g}$ protein vs. $15.35(8.48-42.32) \mu \mathrm{g} / \mathrm{g}$ protein; $P=0.968$; Figure $4.2 \mathrm{~A}]$ nor in sigmoid biopsies [7.56 (1.16-53.55) $\mu \mathrm{g} / \mathrm{g}$ protein vs. 7.61 (1.87-65.57) $\mu \mathrm{g} / \mathrm{g}$ protein; $P=1.000$; Figure 4.2B].

Table 4.4 Glutathione and glutathione disulphide in mucosal biopsies.

\begin{tabular}{lccc}
\hline & Patients with Cirrhosis & Controls & $P$ value \\
\hline Duodenal biopsies ( $\mathrm{n}=12$ vs. $\mathrm{n}=20)$ & & & \\
GSH (nmol/mg protein) & $43.3(36.2-56.3)$ & $42.4(28.5-54.9)$ & 1.000 \\
GSSG (nmol/mg protein) & $0.22(0.05-1.13)$ & $0.18(0.04-0.66)$ & 0.785 \\
Sigmoid biopsies ( $\mathrm{n}=10$ vs. $\mathrm{n}=22)$ & & & \\
GSH (nmol/mg protein) & $29.4(25.2-34.4)$ & $30.3(26.0-42.9)$ & 0.223 \\
GSSG (nmol/mg protein) & $0.17(0.11-0.74)$ & $0.18(0.09-0.64)$ & 0.714 \\
\hline
\end{tabular}

All values are medians (range). GSH, glutathione; GSSG, glutathione disulphide.
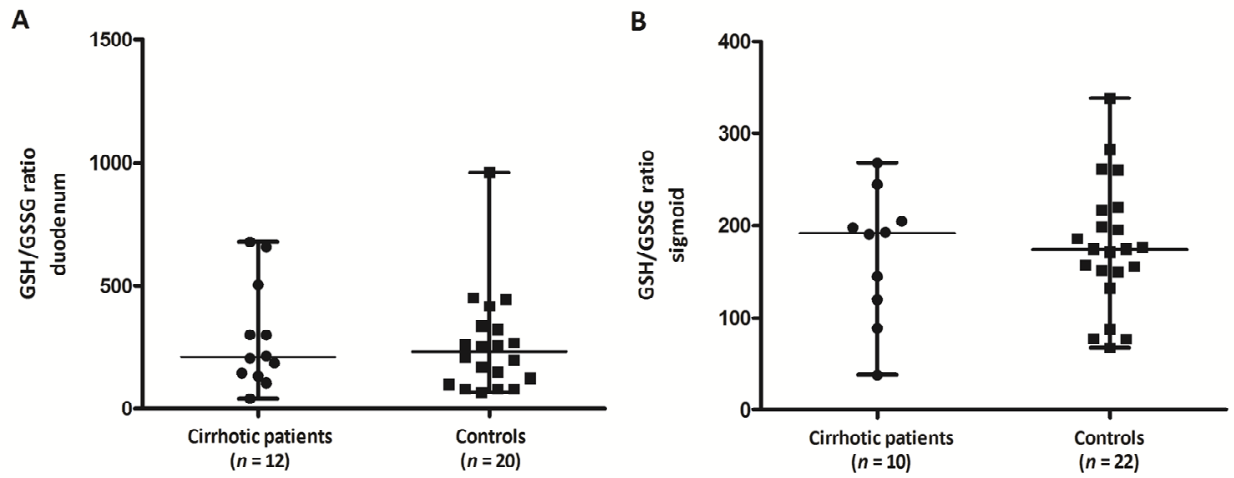

Figure 4.1 Glutathione (GSH) / glutathione disulphide (GSSG) ratio in mucosal biopsies of cirrhotic patients and controls. A: Glutathione (GSH) / glutathione disulphide (GSSG) ratio in duodenal biopsies. B: Glutathione (GSH) / glutathione disulphide (GSSG) ratio in sigmoid biopsies. Data are presented as scatter dot plots displaying median with range $(P>0.05)$.

\section{Fecal calprotectin}

The intestinal inflammation marker fecal calprotectin did also not differ significantly between cirrhotic patients $(n=15)$ and controls $(n=22)$ [6.37 $(0.08-23.46) \mu \mathrm{g} / \mathrm{g}$ feces vs. $1.95(0.13-29.63) \mu \mathrm{g} / \mathrm{g}$ feces; $P=0.216]$. 
A

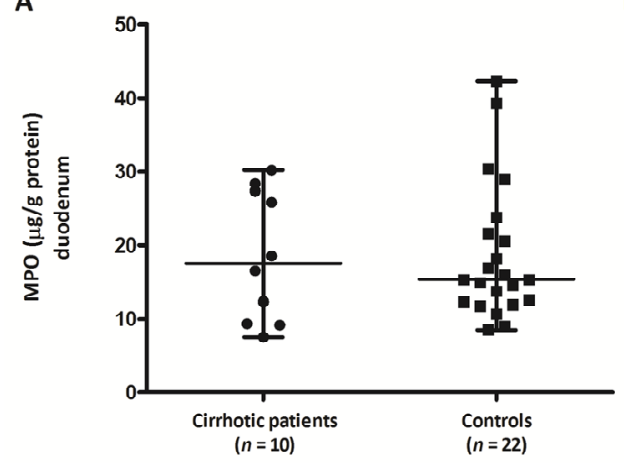

B

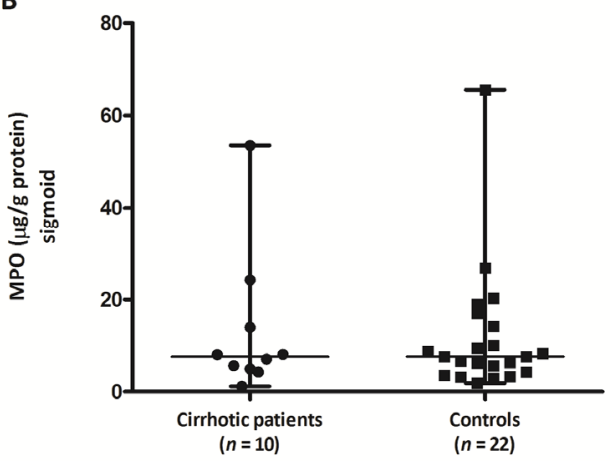

Figure 4.2 Myeloperoxidase (MPO) concentrations in mucosal biopsies of cirrhotic patients and controls. A: Myeloperoxidase (MPO) concentrations in duodenal biopsies. B: Myeloperoxidase (MPO) concentrations in sigmoid biopsies. Data are presented as scatter dot plots displaying median with range $(P>0.05)$.

\section{Histology of mucosal biopsies}

Microscopically, only minor morphological changes were observed in duodenal biopsies of cirrhotic patients and controls, and did not differ significantly between the groups, such as a (slightly) increased number of intra-epithelial lymphocytes ( $n=2$ vs. $n=2$, respectively), mild edema of the lamina propria ( $n=2$ vs. $n=3$, respectively) and mild chronic inflammation ( $n=3$ vs. $n=2$, respectively). None of the subjects had villous atrophy or signs of acute inflammation. In sigmoid biopsies, only mild edema of the lamina propria was observed in both groups ( $n=4$ vs. $n=4$, respectively).

\section{Discussion}

We investigated the occurrence of oxidative stress in both duodenal and sigmoid mucosal biopsies of patients with compensated liver cirrhosis. Differences were only found for gene transcription of GS and GR in duodenal and sigmoid biopsies between compensated cirrhotic patients and healthy controls, but no alterations were found for other genes nor for protein levels of GSH, GSSG and GSH/GSSG ratio. The intestinal inflammation markers MPO and (fecal) calprotectin were also not different between both groups.

The present study was initiated to investigate whether intestinal oxidative stress occurs in patients with compensated liver cirrhosis and thereby could be an underlying mechanism contributing to the epithelial barrier dysfunction observed in our previous study, $^{28}$ which potentially may increase the risk of progression towards decompensated cirrhosis. 
The transcription of genes involved in the metabolism of GSH and uric acid, which are both important antioxidants and protect cells against oxidative damage, were analyzed. GSH reduces hydrogen peroxide and lipid hydroperoxide to less reactive oxygen species (ROS), and in this process GSH is converted into GSSG. Uric acid is produced from hypoxanthine and xanthine by the enzymes $\mathrm{XO}$ and $\mathrm{XDH}$. In vitro, uric acid is a powerful scavenger of $\mathrm{ROS}^{32}$ and by being preferentially oxidized as a substrate for oxidation by haem protein or $\mathrm{H}_{2} \mathrm{O}_{2}$ systems, it can protect against oxidative damage. ${ }^{33}$ As marker of oxidative stress, the GSH/GSSG ratio was determined in both duodenal and sigmoid biopsies.

In the duodenal mucosa, we observed that the gene transcription of $\mathrm{GR}$, the enzyme that recycles GSSG into GSH, was upregulated in cirrhotic patients. Protein levels of GSH, GSSG and the GSH/GSSG ratio were not altered. These findings may point to an enhancement of repair processes for oxidative stress. In the sigmoid mucosa, gene transcription of GR as well as GS, the latter being one of the enzymes involved in the synthesis of GSH, was downregulated in cirrhotic patients, which might indicate a decreased formation of GSH. However, protein levels of GSH, GSSG and the GSH/GSSG ratio were not altered. It should be noted that the differences in gene transcription in both duodenum and sigmoid did not remain statistically significant after correcting for multiple testing and therefore should be interpreted with care. In addition, no alterations were found for the other genes investigated. Thereby the above findings indicate that there is no clear evidence for oxidative stress in the intestine of patients with compensated liver cirrhosis. This is further supported by lack of differences in intestinal MPO and fecal calprotectin concentrations between cirrhotic patients and controls. Moreover, we observed no clear morphological abnormalities in the duodenal and sigmoid biopsies.

The lack of changes in oxidative stress and inflammatory parameters is not likely to be attributed to drug therapy as patients used almost no drugs with antioxidant and/or anti-inflammatory effects and no antioxidant supplements.

Alcohol is an important cause of liver cirrhosis and can disturb intestinal epithelial barrier function by inducing intestinal oxidative damage. ${ }^{16,34}$ In our previous study, small intestinal permeability was found to be increased in a subgroup of patients with alcohol-related cirrhosis, but the small number of patients with alcohol-related cirrhosis $(n=4)$ in the present study precluded us from performing additional analyses to investigate whether this subgroup may also be more susceptible for intestinal oxidative stress.

In contrast to the present findings in humans with compensated liver cirrhosis, some animal studies did provide evidence of oxidative stress in the small and large intestine of rats with carbon tetrachloride $\left(\mathrm{CCl}_{4}\right)$-induced liver cirrhosis, although part of them had ascites. ${ }^{13,24-26}$ Published data on the occurrence of intestinal oxidative stress in cirrhotic patients are scarce. Only one other recent study has investigated intestinal oxidative stress in duodenal biopsies of cirrhotic patients by measuring lipid hydroperoxides and found no difference in the levels of lipid hydroperoxides between 
patients with compensated cirrhosis and healthy controls. ${ }^{27}$ However, the investigators did find significantly increased levels of lipid hydroperoxides in patients with decompensated cirrhosis when compared with those with compensated cirrhosis and healthy controls. Therefore, we cannot exclude that intestinal oxidative stress does occur in patients with decompensated cirrhosis.

The strength of our study is that we investigated the occurrence of oxidative stress not only in the mucosa of the small, but also of the large intestine in patients with compensated cirrhosis. As we previously did find a clear increased large intestinal permeability, it would be interesting to investigate also other potential causative factors contributing to barrier dysfunction, such as changes in microbiota composition. Some limitations of the present study should also be taken into account. The GSH/GSSG ratio was analyzed as this ratio is a marker of oxidative stress and gives insight into the body's anti-oxidant defense capacity. ${ }^{35,36}$ Because of the limited number of intestinal biopsies available per subject, we were not able to measure other markers of oxidative stress, such as MDA and 4-hydroxynonenal (4-HNE). Our findings are supported by unaltered inflammatory parameters and intestinal morphology. Although we cannot completely exclude the presence of intestinal oxidative stress, the current findings indicate that oxidative stress seems not to be a major factor in these patients. Furthermore, the intestinal biopsies could only be obtained from a rather small group of patients due to the invasiveness of sampling and the complexity of the patient population. Therefore, our results are preliminary and subgroup analyses of cirrhotic patients for example with regard to etiology or drug therapy were not possible. Although we cannot exclude the effect of etiology or drug therapy, nor the possibility that other markers of oxidative stress would be increased in cirrhotic patients, we believe that these factors are not important confounders in this study. Furthermore, inclusion of a larger group of patients is not very likely to change our results as no trend to significance was observed in both oxidative stress and inflammatory parameters between cirrhotic patients and controls.

In conclusion, in stable compensated cirrhosis, there were no indications for the occurrence of oxidative stress and low-grade inflammation in both the small and large intestine. Although these preliminary findings need further validation, we found intestinal oxidative stress not to be a major mechanism contributing to epithelial barrier dysfunction observed in patients with compensated cirrhosis. 


\section{References}

1. Misra V, Misra SP, Dwivedi M, Gupta SC. Histomorphometric study of portal hypertensive enteropathy. Am J Clin Pathol 1997;108:652-7.

2. Such J, Guardiola JV, de Juan J, et al. Ultrastructural characteristics of distal duodenum mucosa in patients with cirrhosis. Eur J Gastroenterol Hepatol 2002;14:371-6.

3. Chesta J, Defilippi C. Abnormalities in proximal small bowel motility in patients with cirrhosis. Hepatology 1993;17:828-32.

4. Chen $\mathrm{Y}$, Yang F, Lu H, et al. Characterization of fecal microbial communities in patients with liver cirrhosis. Hepatology 2011;54:562-72.

5. Campillo B, Pernet P, Bories PN, Richardet JP, Devanlay M, Aussel C. Intestinal permeability in liver cirrhosis: relationship with severe septic complications. Eur J Gastroenterol Hepatol 1999;11:755-9.

6. Zuckerman MJ, Menzies IS, Ho H, et al. Assessment of Intestinal Permeability and Absorption in Cirrhotic Patients with Ascites Using Combined Sugar Probes. Dig Dis Sci 2004;49:621-6.

7. Norman K, Pirlich M, Schulzke JD, et al. Increased intestinal permeability in malnourished patients with liver cirrhosis. Eur J Clin Nutr 2012;66:1116-9.

8. Bellot $P$, Frances $R$, Such J. Pathological bacterial translocation in cirrhosis: pathophysiology, diagnosis and clinical implications. Liver Int 2013;33:31-9.

9. Wiest R, Garcia-Tsao G. Bacterial translocation (BT) in cirrhosis. Hepatology 2005;41:422-33.

10. Assimakopoulos SF, Tsamandas AC, Tsiaoussis GI, et al. Altered intestinal tight junctions' expression in patients with liver cirrhosis: a pathogenetic mechanism of intestinal hyperpermeability. Eur J Clin Invest 2012;42:439-46.

11. Du Plessis J, Vanheel H, Janssen CE, et al. Activated intestinal macrophages in patients with cirrhosis release NO and IL-6 that may disrupt intestinal barrier function. J Hepatol 2013;58:1125-32.

12. Rao RK, Basuroy S, Rao VU, Karnaky Jr KJ, Gupta A. Tyrosine phosphorylation and dissociation of occludin-ZO-1 and E-cadherin-beta-catenin complexes from the cytoskeleton by oxidative stress. Biochem J 2002;368:471-81.

13. Ramachandran A, Prabhu R, Thomas S, Reddy JB, Pulimood A, Balasubramanian KA. Intestinal mucosal alterations in experimental cirrhosis in the rat: role of oxygen free radicals. Hepatology 2002;35:622-9.

14. Sheth P, Basuroy S, Li C, Naren AP, Rao RK. Role of phosphatidylinositol 3-kinase in oxidative stressinduced disruption of tight junctions. J Biol Chem 2003;278:49239-45.

15. Assimakopoulos SF, Gogos C, Labropoulou-Karatza C. Could antioxidants be the "magic pill" for cirrhosis-related complications? A pathophysiological appraisal. Med Hypotheses 2011;77:419-23.

16. Banan A, Choudhary S, Zhang Y, Fields JZ, Keshavarzian A. Ethanol-induced barrier dysfunction and its prevention by growth factors in human intestinal monolayers: evidence for oxidative and cytoskeletal mechanisms. J Pharmacol Exp Ther 1999;291:1075-85.

17. Forsythe RM, Xu DZ, Lu Q, Deitch EA. Lipopolysaccharide-induced enterocyte-derived nitric oxide induces intestinal monolayer permeability in an autocrine fashion. Shock 2002;17:180-4.

18. Bajaj JS, Hylemon PB, Ridlon JM, et al. Colonic mucosal microbiome differs from stool microbiome in cirrhosis and hepatic encephalopathy and is linked to cognition and inflammation. Am J Physiol Gastrointest Liver Physiol 2012;303:G675-85.

19. Saitoh O, Sugi K, Lojima K, et al. Increased prevalence of intestinal inflammation in patients with liver cirrhosis. World J Gastroenterol 1999;5:391-6.

20. Iwao T, Toyonaga A, Ikegami M, et al. Reduced gastric mucosal blood flow in patients with portalhypertensive gastropathy. Hepatology 1993;18:36-40.

21. $\mathrm{Xu} \mathrm{WH}, \mathrm{Wu} \mathrm{XJ}$, Li JS. Influence of portal pressure change on intestinal permeability in patients with portal hypertension. Hepatobiliary Pancreat Dis Int 2002;1:510-4.

22. Ramachandran A, Balasubramanian KA. Intestinal dysfunction in liver cirrhosis: Its role in spontaneous bacterial peritonitis. J Gastroenterol Hepatol 2001;16:607-12.

23. Zuwala-Jagiello J, Pazgan-Simon M, Simon K, Warwas M. Elevated advanced oxidation protein products levels in patients with liver cirrhosis. Acta Biochim Pol 2009;56:679-85.

24. Chiva M, Soriano G, Rochat I, et al. Effect of Lactobacillus johnsonii La1 and antioxidants on intestinal flora and bacterial translocation in rats with experimental cirrhosis. J Hepatol 2002;37:456-62. 
25. Chiva M, Guarner C, Peralta C, et al. Intestinal mucosal oxidative damage and bacterial translocation in cirrhotic rats. Eur J Gastroenterol Hepatol 2003;15:145-50.

26. Natarajan SK, Ramamoorthy $\mathrm{P}$, Thomas $\mathrm{S}$, et al. Intestinal mucosal alterations in rats with carbon tetrachloride-induced cirrhosis: changes in glycosylation and luminal bacteria. Hepatology 2006;43: 837-46.

27. Assimakopoulos SF, Tsamandas AC, Tsiaoussis GI, et al. Intestinal mucosal proliferation, apoptosis and oxidative stress in patients with liver cirrhosis. Ann Hepatol 2013;12:301-7.

28. Pijls KE, Koek GH, Elamin EE, de Vries H, Masclee AA, Jonkers DM. Large intestine permeability is increased in patients with compensated liver cirrhosis. Am J Physiol Gastrointest Liver Physiol 2014; 306:G147-53.

29. Julicher RH, Sterrenberg L, Haenen GR, Bast A, Noordhoek J. The effect of chronic adriamycin treatment on heart kidney and liver tissue of male and female rat. Arch Toxicol 1988;61:275-81.

30. van der Sluijs Veer G, van den Hoven B, Russel MG, van den Bergh FA. Time-resolved fluorimetric immunoassay of calprotectin: technical and clinical aspects in diagnosis of inflammatory bowel diseases. Clin Chem Lab Med 2006;44:292-8.

31. de Haan JJ, Lubbers $T$, Hadfoune $M$, et al. Postshock intervention with high-lipid enteral nutrition reduces inflammation and tissue damage. Ann Surg 2008;248:842-8.

32. Ames BN, Cathcart R, Schwiers E, Hochstein P. Uric acid provides an antioxidant defense in humans against oxidant- and radical-caused aging and cancer: a hypothesis. Proc Natl Acad Sci U S A 1981;78: 6858-62.

33. Halliwell B, JMC G. Free radicals in biology and medicine: New York: Oxford University Press Inc.; 1999.

34. Banan A, Fields JZ, Decker H, Zhang Y, Keshavarzian A. Nitric oxide and its metabolites mediate ethanol-induced microtubule disruption and intestinal barrier dysfunction. J Pharmacol Exp Ther 2000; 294:997-1008.

35. Sen SK, Packer L, O H. Handbook of Oxidants and Antioxidants in Exercise: Elsevir Science B.V.; 2000.

36. Koek GH, Liedorp PR, Bast A. The role of oxidative stress in non-alcoholic steatohepatitis. Clin Chim Acta 2011;412:1297-305. 
74 


\section{Chapter 5}

Altered fecal and duodenal microbial profiles are associated with disease severity in liver cirrhosis

patients

K.E. Pijls*

D. Tedjo*

G.H. Koek

A.E. Budding

P.H.M. Savelkoul

A.A.M. Masclee

J. Penders

D.M.A.E. Jonkers

*Both authors contributed equally to this work

Submitted 


\section{Abstract}

Increasing data show perturbations in the fecal microbiota of liver cirrhosis patients. Whether these findings can be useful in daily clinical practice needs further study. Furthermore, mucosal microbiota composition data are limited. The 16S-23S interspacer region-based profiling method (IS-pro) has potential as clinical tool to assess and monitor intestinal microbiota. The aim of this study was to investigate the fecal and mucosa-associated microbiota in a heterogeneous group of (de)compensated cirrhosis patients and healthy controls, taking into account disease-related factors.

Feces, sigmoid and duodenum biopsies of healthy controls ( $n=26, n=22$ and $n=18$, resp.), compensated ( $n=25, n=12$ and $n=13$, resp.) and feces of decompensated cirrhotic patients ( $n=16)$ were available for analysis. Microbiota composition was assessed by IS-pro.

Feces showed significant higher richness [median (range)] for Firmicutes in compensated [5.0 (0.9-12.0)] and decompensated cirrhotics [6.5 (1.0-22.0)] versus controls [3.0 (0.0-10.0), all $P<0.05]$. Lower richness for Bacteroidetes was found in compensated [20.0 (4.0-39.0)] and decompensated cirrhotics [18.5 (0.0-26.0)] versus controls [23.5 (13-32), all $P<0.05]$. In duodenum, only an increased richness for Firmicutes was found in compensated cirrhotics [10.0 (3.0-13.0)] versus controls [5.0 (0.0-14.0), $P=0.01]$. No significant microbial differences were found in sigmoid. Constrained ordination showed distinct fecal clusters based on disease severity by Child-Pugh classification, but not by demographics, etiology or drug therapy.

Differences in the fecal and duodenal, but not sigmoid microbiota were found between cirrhotics and controls. Alterations in the fecal microbiota were more pronounced with progression of liver cirrhosis. Distinct clusters based on Child-Pugh classification, support the potential of IS-profiling as disease progression marker. 


\section{Introduction}

The intestinal microbiota is a complex ecosystem colonizing the entire length of the intestine. At phylum level, the microbiota is dominated by two phyla, i.e. Firmicutes and Bacteriodetes, whereas at lower taxonomic levels the microbiota consists of more than 1000 different species. ${ }^{1,2}$ Increasing evidence indicates that the intestinal microbiota is involved in a variety of not only intestinal, but also systemic and liver diseases. ${ }^{3-5}$ Microbial perturbations have been reported in patients with chronic liver diseases, such as non-alcoholic fatty liver disease (NAFLD) ${ }^{6}$ and alcoholic liver disease (ALD), ${ }^{7}$ diseases that can progress to liver cirrhosis. Liver cirrhosis is the end stage of chronic liver diseases and can be divided into an asymptomatic stage of compensated cirrhosis and a symptomatic stage of decompensated cirrhosis. The latter is defined by the development of clinically evident complications, i.e. ascites, variceal hemorrhage, hepatic encephalopathy and/or jaundice. ${ }^{8}$ Further insight in underlying mechanisms contributing to disease progression, both hepatic and extra-hepatic, such as alterations in the intestinal microbiota and intestinal permeability, as well as identification of noninvasive markers thereof, are relevant for early diagnosis and to identify targets for interventions.

In recent years, several studies characterized the structure and function of the fecal microbiota in patients with compensated and/or decompensated cirrhosis in comparison with healthy controls. An increase of Proteobacteria and Fusobacteria, and a corresponding decrease of Bacteroidetes in mixed groups of patients with compensated and decompensated cirrhosis versus healthy controls have been reported in several studies. ${ }^{9-11}$ At family level, Enterobacteriaceae, ${ }^{10-12}$ Streptococcaceae ${ }^{10,11,13}$ and Veillonellaceae $e^{10,11,13,14}$ were often found to be increased, whereas Ruminococcaceae $e^{12,14}$ and Lachnosperiaceae ${ }^{11,12,15}$ were decreased in patients with cirrhosis. The alterations in the microbiota composition seem to be more pronounced in patients with advanced cirrhosis, especially in those with complications, such as hepatic encephalopathy. ${ }^{16,17}$ Whether these findings are relevant for daily clinical practice and care, needs further study.

The in-depth analysis of the intestinal microbiota has predominantly been performed with sequencing approaches, requiring time-consuming and expert bioinformatics support. Although 16S rRNA gene sequencing is state-of-the-art in the research setting, this method is not readily applicable in routine clinical practice for on demand analyses. The validated 16S-23S interspacer (IS) region-based profiling method (IS-pro) may have potential as a clinical tool to assess and monitor the intestinal microbiota structure, $^{18}$ as this method enables relatively fast analysis of individual samples. Furthermore, abovementioned studies have focused primarily on the fecal microbiota in cirrhotic patients with alcohol and/or viral infections as its main cause. Data on mucosa-associated microbiota and cirrhotic patients with mixed etiologies are limited. The aim of the present study was to investigate differences in the fecal and the mucosa-associated microbiota in a heterogeneous group of patients with 
compensated cirrhosis versus controls, taking into account disease-related factors by use of IS-profiling. In addition, the fecal microbiota was assessed in patients with decompensated cirrhosis.

\section{Materials and methods}

\section{Patients and study design}

Patients with stable compensated liver cirrhosis of various etiology (i.e. without clinically evident complications, including ascites, variceal hemorrhage, hepatic encephalopathy and/or jaundice) and age, sex and BMI-matched healthy volunteers participating in a prior prospective case-control study on intestinal permeability, were available for analyses of the intestinal microbiota. ${ }^{19}$ In addition to biological samples, extensive demographic and clinical characteristics (i.e. severity, etiology and drug therapy) were available, as well as urinary sugar excretions as indicators of small [lactulose/rhamnose (L/R) ratio] and large intestinal permeability [sucralose/erythritol (S/E) ratio].

Briefly, 26 compensated liver cirrhosis patients (CLC) and 27 healthy controls (HC) collected a fresh fecal sample. Aliquots were stored within 12 hours after defecation at $-80^{\circ} \mathrm{C}$ until further analysis. A subset of patients $(n=15)$ and controls $(n=22)$ underwent a gastroduodenoscopy and/or sigmoidoscopy after an overnight fast, but without prior bowel cleansing. The gastroduodenoscopy and sigmoidoscopy preparation and procedures were similar for patients and controls. Mucosal biopsies were obtained from standardized locations: from the second segment of the duodenum and from the sigmoid approximately $20 \mathrm{~cm}$ from the anal sphincter. Biopsies for microbiological analyses were snap-frozen in liquid nitrogen and stored at $-80^{\circ} \mathrm{C}$ until further processing.

In addition, fresh frozen fecal samples were available from a group of inpatients with decompensated liver cirrhosis (DLC) $(n=16)$. Decompensated cirrhosis was defined by the presence of one or more of the following clinically evident complications: ascites, variceal hemorrhage, hepatic encephalopathy and/or jaundice. Severity of liver cirrhosis of compensated and decompensated patients was scored according to the Child-Pugh classification. ${ }^{20}$

The study has been approved by the Medical Ethics Committee of Maastricht University Medical Center (MUMC), was conducted according to the revised version of the Declaration of Helsinki (October 2008, Seoul) and registered at the US National Library of Medicine (http://www.clinicaltrials.gov, NTC01081236). All subjects (patients and healthy volunteers) gave their written informed consent prior to participation. 


\section{DNA isolation}

Frozen fecal samples were kept at $0^{\circ} \mathrm{C}$, and $500 \mu$ lysis buffer (Biomérieux, Marcy l'Etoile, France) was added to approximately $250 \mathrm{mg}$ of the fecal samples. The samples were then vortexed and shaken for $5 \mathrm{~min}$ at room temperature (RT). Subsequently, the samples were centrifuged for $2 \mathrm{~min}$ at 14,000 rpm and $100 \mu$ l supernatant was used for the subsequent isolation procedure. Two $\mathrm{ml}$ lysis buffer (Biomérieux, Marcy I'Etoile, France) was added to the supernatant. The samples were incubated for $10 \mathrm{~min}$ at RT and $70 \mu \mathrm{l}$ magnetic silica beads (Biomérieux, Marcy l'Etoile, France) were added. The DNA isolation was performed with the NucliSENS ${ }^{\circledR}$ easyMag ${ }^{\circledR}$ automated DNA isolation machine (Biomérieux, Marcy l'Etoile, France) with the Specific A protocol as described by the manufacturer. DNA was eluted in $110 \mu$ l elution buffer (Biomérieux, Marcy l'Etoile, France).

Duodenal and sigmoid biopsies were thawed on RT, and $360 \mu$ ATL buffer (Qiagen, Hilden, Germany) and $40 \mu$ l proteinase K (Qiagen, Hilden, Germany) were added to each biopsy specimen. The samples were shaken at maximum speed for 1 hour at $56^{\circ} \mathrm{C}$. Subsequently, $400 \mu \mathrm{l}$ AL buffer (Qiagen, Hilden, Germany) was added and the samples were vortexed for 15 seconds and incubated for $10 \mathrm{~min}$ at $70^{\circ} \mathrm{C}$. Samples were centrifuged for $2 \mathrm{~min}$ at 18,000 $\mathrm{rcf}$ and the complete supernatant was used for the subsequent isolation procedure. Two $\mathrm{ml}$ lysis buffer (Biomérieux, Marcy l'Etoile, France) was added to supernatants and the samples were incubated for $10 \mathrm{~min}$ at RT. Seventy $\mu$ magnetic silica beads (Biomérieux, Marcy l'Etoile, France) were added to each sample. The DNA isolation was performed with the NucliSENS ${ }^{\circledR}$ easyMag $^{\circledR}$ automated DNA isolation machine (Biomérieux, Marcy l'Etoile, France) with the Specific A protocol as described by the manufacturer.

\section{S-23S IS profiling of the intestinal microbiota}

IS-pro is a validated PCR based bacterial profiling technique, ${ }^{18}$ which combines species differentiation by the 16S-23S rRNA intergenic spacer (IS) region with taxonomic classification by the phylum-specific fluorescent labeled primers. The IS-pro technique uses 2 multiplex PCRs to detect the phyla Firmicutes, Actinobacteria, Fusobacteria, Verrucomicrobia, Bacteroidetes and Proteobacteria. The first PCR reaction consists of 3 unlabeled reverse primers and 2 different fluorescently labeled forward primers (one specific for Firmicutes, Actinobacteria, Fusobacteria and Verrucomicrobia and one specific for Bacteroidetes). The second PCR consists of a combination of 7 reverse primers and one forward labeled primer specific for Proteobacteria. Amplification of the 16S-23S rRNA IS-regions was performed with the IS-pro assay (IS-Diagnostics, Amsterdam, the Netherlands) according to the protocol provided by the manufacturer on a GeneAmp PCR system 9700 (Applied Biosystems, Foster City, CA, USA). ${ }^{18}$ To analyze the DNA fragments, $5 \mu \mathrm{l}$ PCR product was added to $19.8 \mu \mathrm{l}$ formamide and $0.2 \mu \mathrm{l}$ MapMarker 1000 ROX-labeled size marker (Bioventures, Murfreesboro, TN, 
USA). DNA fragment analysis was performed on an ABI Prism 3130xI Genetic Analyzer (Applied Biosystems).

\section{Data analyses}

Subject characteristics are presented as median (range) and were compared between groups using the Mann-Whitney $U$-test for non-parametric data in case of continuous variables or with the $\chi^{2}$ test in case of dichotomous variables. A $P$ value $<0.05$ was considered statically significant using a two-tailed test.

The IS-pro data consists of peaks with a specific length and its height [relative fluorescence units (RFU)] corresponding to the abundance. All RFUs were log2 transformed for downstream analysis to reduce the dominance of high peaks and to include less abundant species. A heatmap was made based on all IS-profiles and stratified by phyla using the Spotfire package (TIBCO, Palo Alto, CA, USA). The observed peaks, Shannon index and Bray-Curtis (beta-diversity) dissimilarity were calculated as described previously. ${ }^{21}$

The Mann-Whitney $U$-test was used to analyze significant differences in Firmicutes/Bacteroidetes (F/B) ratio, numbers of observed peaks (richness) and the Shannon diversity index (diversity) between $\mathrm{HC}$ and CLC and DLC, using SPSS version 20.0. To test for significant differences in the peak frequency between $\mathrm{HC}$ and $\mathrm{CLC}$, the Kruskal-Wallis test was applied. To correct for multiple testing a false discovery rate (FDR) of q-value of $\leq 0.15$ was used as a cut-off value.

A Pearson product-moment correlation analysis was performed to investigate the relationship between sugar ratios (S/E and L/R) as markers of intestinal permeability, and peaks with a significantly different frequency between HC and CLC according to the Kruskal-Wallis test. A FDR of 0.05 was used as a cut-off value.

The non-parametric t-test (using Monte Carlo permutations to calculate the significance in Qiime 1.9) was used to compare the microbiota structure between HC and CLC.

Principle coordinate analysis (PCOA) plots of Bray-Curtis dissimilarities were constructed to visualize differences in microbiota community structure of $\mathrm{HC}$ versus CLC. To analyze the effect of disease status, sex, age, BMI or smoking on the microbiota community structure, a distance-based redundancy analysis (db-RDA) was performed on the Bray-Curtis distance matrix using the capscale function in the vegan package of R. db-RDA is a constrained ordination method and consists of applying PCOA on the dataset and analyzing the output with RDA in which multiple linear regressions are performed in an iterative way, to find the best ordination of samples. An ANOVA-like permutation test was subsequently applied to determine statistical significance of the individual variables. An additional db-RDA was performed on the fecal samples and mucosal biopsies of CLC, to investigate whether disease severity, etiology and drug therapy had an effect on the overall microbiota structure. 
Partial least squares discriminant analysis (PLS-DA) was used to build a model to predict disease severity as defined by the Child-Pugh class based on the Bray-Curtis dissimilarity for each separate phylum and for all phyla combined. This is a supervised method, using both the dependent (Child-Pugh class) and independent variables (peaks) for differentiation. Validation of the model was done by 10 -fold cross validation, in which $90 \%$ of the data was used to train the model (training set), and the remaining $10 \%$ to validate the model (validation set). This was repeated for 10 iterations, in which each sample was used as a test sample once. During the PLS-DA procedure Variable Importance in the Projection (VIPs) are computed for each variable (i.e. peaks) and provides an estimation of discriminative power of the variables in which a high number indicates a high discriminative power. PLS-DA as described above was done for a subset of peaks with a VIP score $>1$ and peaks with a VIP score $>2.3$ to improve the discriminative power of the model. PLS-DA was performed in Spotfire (TIBCO, Palo Alto, CA, USA).

\section{Results}

\section{Patients}

Fecal samples were obtained from $27 \mathrm{HC}, 26 \mathrm{CLC}$ and 16 DLC patients. Serum alanine transaminase (ALT) and gamma-glutamyl transferase (GGT) were significantly increased in patients with CLC versus HC (ALT; $P=0.025$, GGT; $P<0.001)$. Furthermore, 17 and 9 CLC patients were classified as Child-Pugh class $A$ and $B$, respectively.

DLC patients had significantly higher serum ALT and GGT levels than HC (ALT; $P=0.002$, GGT; $P<0.001)$. Comparing DLC with CLC patients, serum GGT was significantly increased in DLC $(P=0.042)$. Furthermore, the Child-Pugh and the MELD score were significantly higher in DLC versus CLC $(P<0.001)$. Subject characteristics including cause of liver cirrhosis and current drug therapy are given in Table 5.1.

\section{IS-pro data}

First, F/B ratios were compared between patients with $\mathrm{CLC}$ and $\mathrm{HC}$, being significantly higher in fecal samples $(P=0.004$, Figure $5.1 \mathrm{~A})$ and duodenal biopsies $(P=0.039$, Figure 5.1C) of CLC, while no differences were observed in sigmoid mucosa ( $P>0.05$, Figure 5.1B) between CLC and HC. The F/B ratio in fecal samples was also significantly higher in DLC versus HC $(P<0.001)$, but not when compared to CLC (Figure 5.1A). 
Table 5.1 Characteristics of subjects.

\begin{tabular}{|c|c|c|c|}
\hline & $\begin{array}{l}\text { Healthy controls } \\
\qquad(n=27)\end{array}$ & $\begin{array}{c}\text { Patients with } \\
\text { compensated cirrhosis } \\
(n=26)\end{array}$ & $\begin{array}{c}\text { Patients with } \\
\text { decompensated cirrhosis } \\
(n=16)\end{array}$ \\
\hline Age (years) & $60(19-78)$ & $61(18-72)$ & $59(39-81)$ \\
\hline $\operatorname{Sex}(M / F)$ & $17 / 10$ & $17 / 9$ & $12 / 4$ \\
\hline BMI $\left(\mathrm{kg} / \mathrm{m}^{2}\right)$ & $26.0(18.1-32.1)$ & $25.8(18.8-39.0)$ & $26.7(20.1-40.0)$ \\
\hline Current smokers & 3 & $10 *$ & $8 *$ \\
\hline $\operatorname{ALT}(U / I)$ & $22.0(10.0-32.0)$ & $28.0(9.0-130.0)^{*}$ & $35.0(14.0-137.0)^{*}$ \\
\hline GGT (U/I) & $22.0(6.0-50.0)$ & $51.0(17.0-259.0) * * *$ & $80.0(29.0-1890.0)^{*}$ \\
\hline \multicolumn{4}{|l|}{ Etiology: } \\
\hline - alcohol-related & & 11 & 12 \\
\hline - autoimmune-related & & 6 & 1 \\
\hline - metabolic & & 2 & 2 \\
\hline - chronic viral infection & & 1 & 1 \\
\hline - cryptogenic & & 5 & 0 \\
\hline - multifactorial & & 1 & 0 \\
\hline Child Pugh class $(\mathrm{A} / \mathrm{B} / \mathrm{C})$ & - & $17 / 9 / 0$ & $0 / 7 / 9$ \\
\hline Child-Pugh score $^{a}$ & - & $5.0(5.0-9.0)^{* *}$ & $10.0(7.0-13.0)$ \\
\hline MELD-score & - & $8.0(6.0-15.0)^{* *}$ & $13.0(7.0-22.0)$ \\
\hline \multicolumn{4}{|l|}{ Drug therapy } \\
\hline - antibiotics & & 1 & 10 \\
\hline - pre/probiotics & & 4 & 2 \\
\hline - laxatives & & 7 & 7 \\
\hline - proton pump inhibitors & & 15 & 9 \\
\hline - ursodeoxycholic acid & & 11 & 2 \\
\hline - glucocorticosteroids/ & & 4 & 1 \\
\hline \multicolumn{4}{|l|}{ immunosuppressives } \\
\hline Liver biopsy & - & 16 & 5 \\
\hline $\mathrm{S} / \mathrm{E}$ ratio in $5-24 \mathrm{~h}$ urine & $(0.008-0.034)$ & $0.019(0.008-0.051)^{*}$ & - \\
\hline $\mathrm{L} / \mathrm{R}$ ratio in $-5 \mathrm{~h}$ urine & $0.023(0.006-0.069)$ & $0.023(0.010-0.115)$ & \\
\hline
\end{tabular}

* $P<0.05$ compared to healthy controls; ${ }^{* *} P<0.05$ compared to patients with decompensated cirrhosis. ${ }^{\text {a }}$ One patient with compensated cirrhosis had a Child-Pugh score of 9 , which was attributed to a high bilirubin level because of Gilbert's Syndrome. L/R, lactulose/rhamnose ratio as indicator for small intestinal permeability; S/E, sucralose/erythritol ratio as indicator for colon permeability. 
A

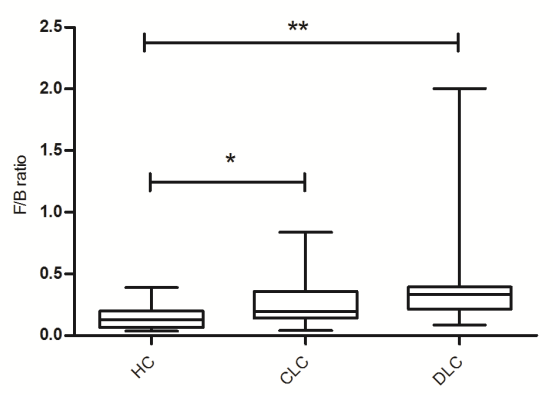

B

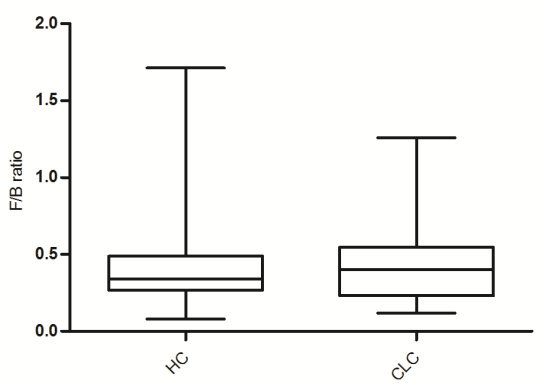

C

Duodenum

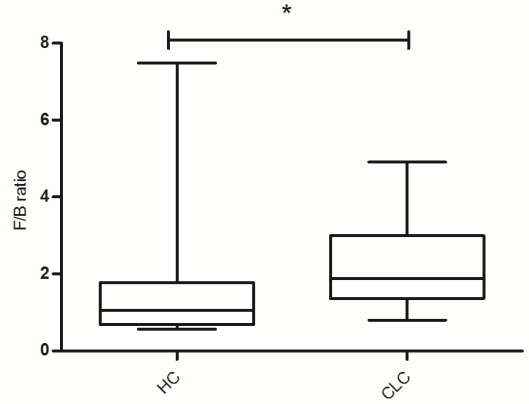

Figure 5.1 Firmicutes/Bacteroidetes ratios of healthy controls $(\mathrm{HC})$, patients with compensated (CLC) and decompensated cirrhosis (DLC). A: Firmicutes/Bacteroidetes ratio in fecal samples. B: Firmicutes/Bacteroidetes ratio in sigmoid biopsies. C: Firmicutes/Bacteroidetes ratio in duodenal biopsies. Data are presented as boxplots displaying median with range $\left({ }^{*} P<0.05, * *\right.$ $P<0.01)$.

\section{Microbial richness and diversity in feces and mucosal biopsies}

In fecal samples, total bacterial richness and diversity were similar between CLC patients and HC, as indicated by the number of observed peaks (Figure 5.2) and the Shannon index, respectively. Both richness and diversity of Firmicutes were significantly higher in CLC than in $\mathrm{HC}(P=0.03$ and $P=0.02$, respectively). In contrast, the richness, but not diversity, of Bacteroidetes was significantly lower in CLC versus HC $(P=0.04)$. No differences were found with regard to the Proteobacteria. The observed differences with regard to species richness and diversity in feces were even more pronounced in DLC (Figure 5.2). 


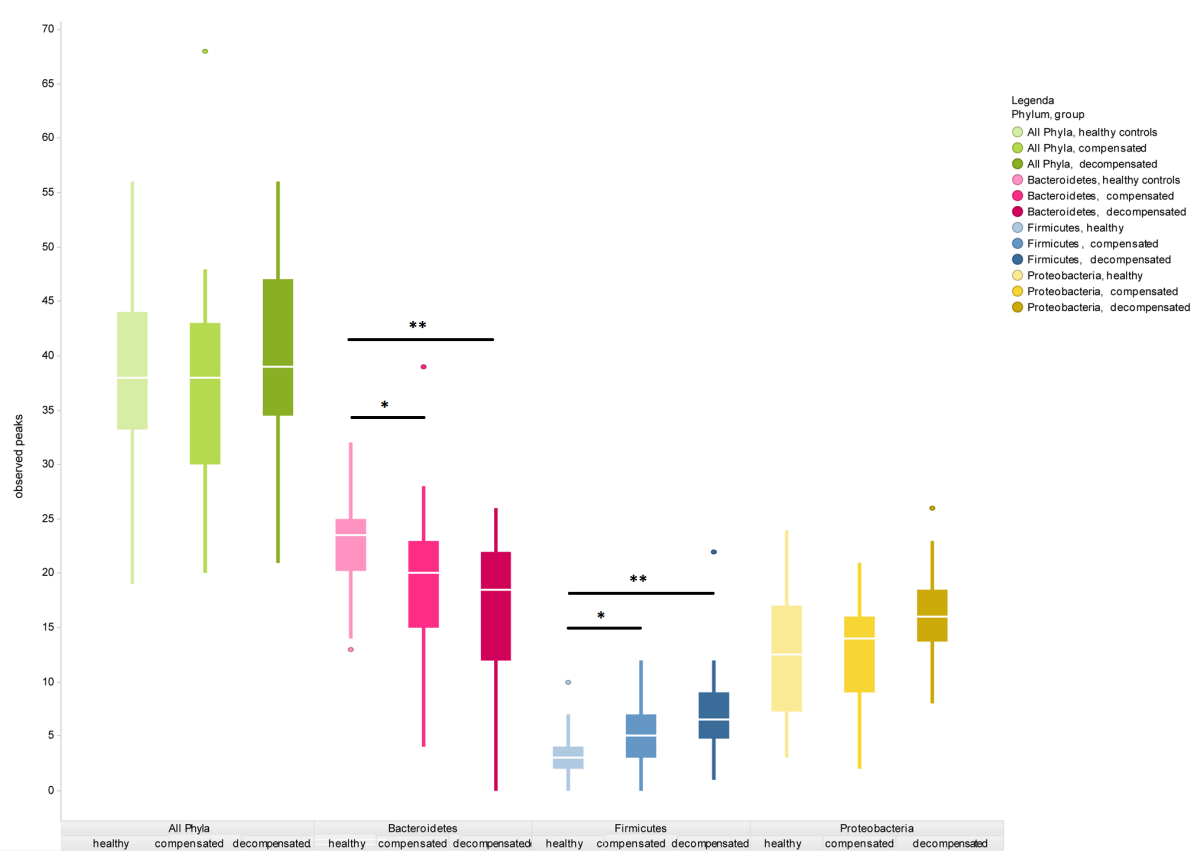

Figure 5.2 Species richness (number of observed peaks) in fecal samples of healthy controls, patients with compensated cirrhosis (CLC) and patients with decompensated cirrhosis (DLC) of all phyla combined (green), Bacteroidetes (pink), Firmicutes (blue) and Proteobacteria (yellow). Data are presented as boxplots displaying median with interquartile range and outliers $\left({ }^{*} P<0.05\right.$, $* * P<0.01)$.

Duodenal and sigmoid biopsies were available from CLC patients and HC. Bacterial richness and diversity of sigmoid biopsies did not differ significantly between CLC and HC (data not shown). In duodenal biopsies, however, Firmicutes richness was significantly increased in CLC versus HC ( $P=0.01$, Figure 5.3), whereas no differences were found with respect to the diversity, nor for richness and diversity of the total bacterial profiles nor for the phyla Bacteroidetes and Proteobacteria.

\section{Fecal microbial community structure}

A heat map was generated including all fecal IS-profiles of subjects with CLC, DLC and $\mathrm{HC}$. Hierarchical clustering indicated that the fecal microbiota composition of patients with cirrhosis and HC were different (Figure 5.4). PCoA comparisons of Bray-Curtis dissimilarities in fecal samples between $\mathrm{HC}$ and CLC showed clustering of HC (Figure S5.1). The median (range) within-group Bray-Curtis dissimilarity was significantly larger for CLC patients [0.58 (0.41-0.79)] than the within-group Bray-Curtis dissimilarity of HC [0.53 (0.38-0.75)], indicating that inter-individual variations in microbiota composition 
of $\mathrm{HC}$ was significantly smaller than the variations in microbiota composition within the group of $\operatorname{CLC}(P=0.01)$.

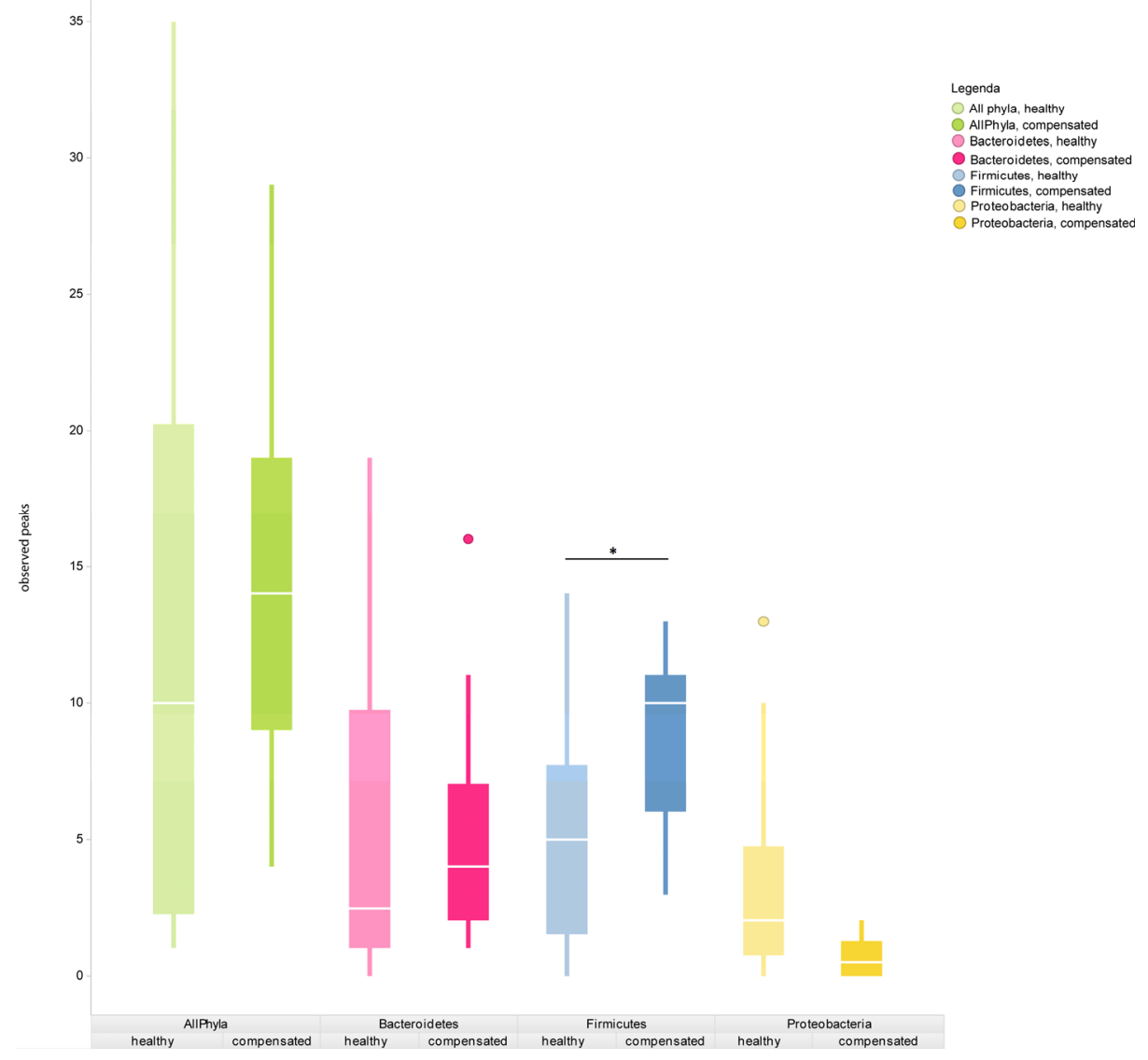

Figure 5.3 Species richness (number of observed peaks) in duodenal biopsies of healthy controls and patients with compensated cirrhosis (CLC) of all phyla combined (green), Bacteroidetes (pink), Firmicutes (blue) and Proteobacteria (yellow). Data are presented as boxplots displaying median with interquartile range and outliers ( $\left.{ }^{*} P<0.05, * * P<0.01\right)$. 


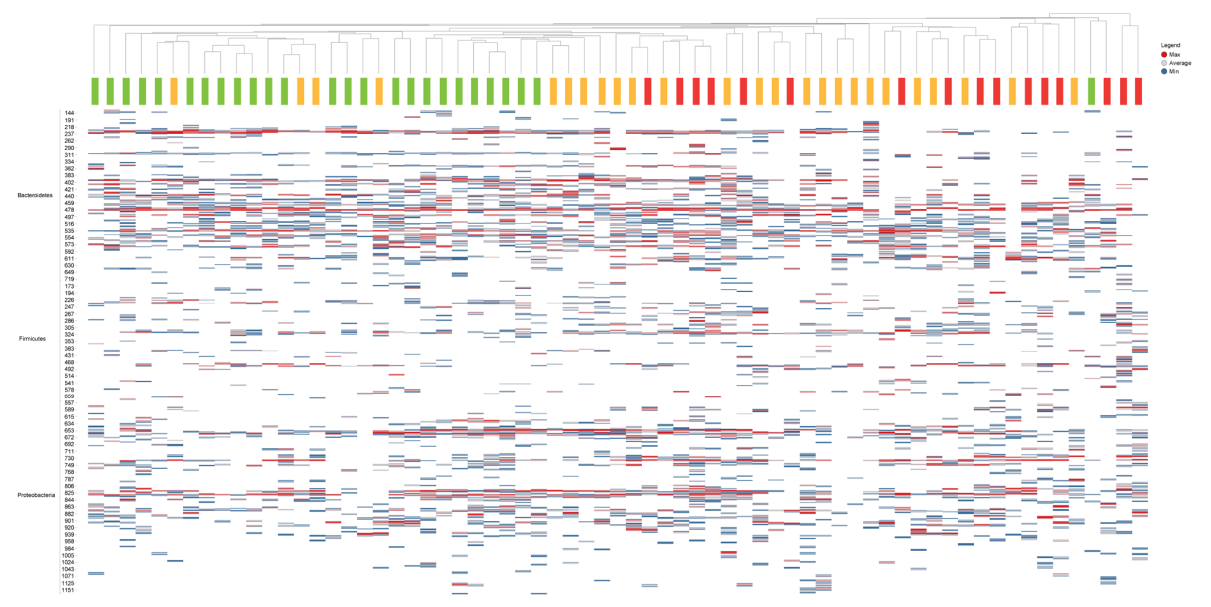

Figure 5.4 Heat map of IS-profiles from all fecal samples of healthy controls (green) and patients with compensated (CLC) (yellow) and decompensated cirrhosis (DLC) (red). Rows are sorted on phylum level and clustering was performed by Unpaired Pair Group Method with Arithmetic Mean (UPGMA).

To determine which host-related factors had a significant effect on the microbiota composition, db-RDA of the Bray-Curtis dissimilarity was performed. This demonstrated that the fecal samples were separated by health status (i.e. CLC vs HC, $P=0.001)$ (Figure 5.5A), but not by age $(P=0.216)$, sex $(P=0.412), \mathrm{BMI}(P=0.052)$, smoking $(P=0.084)$ or alcohol use $(P=0.667)$.

Additionally, db-RDA was employed on CLC patients to explore the effect of disease severity, etiology and drug therapy on the microbiota composition (Figure 5.5D-F), showing that disease severity had a significant effect on the fecal microbiota (ChildPugh class, $P=0.010)$, but not MELD-score $(P=0.312)$, ALT $(P=0.066)$, GGT $(P=0.461)$, etiology $(P=0.756)$ and drug therapy, i.e. PPIs $(P=0.395)$ and laxatives $(P=0.222)$ (Figure 5.5D).

Although no clear separation was seen between CLC and DLC patients by hierarchical clustering, db-RDA showed distinct clusters based on disease severity [HC, patients with Child-Pugh A, B and C cirrhosis (Child-Pugh class, ANOVA $P=0.001$ )]. Fecal samples of cirrhotic patients were more distant to $\mathrm{HC}$ when categorized in a more severe class, suggesting a stronger dysbiosis of the fecal microbiota with progression of liver cirrhosis (Figure 5.6). 
A

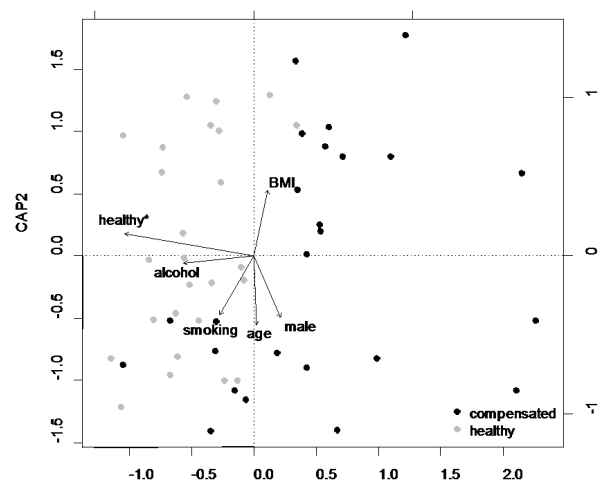

C

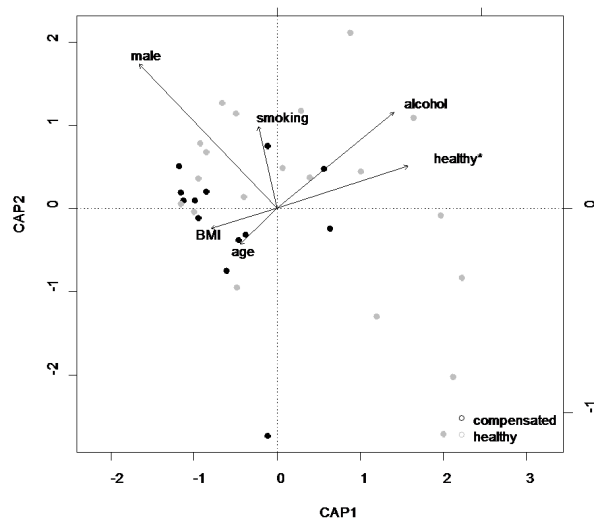

E

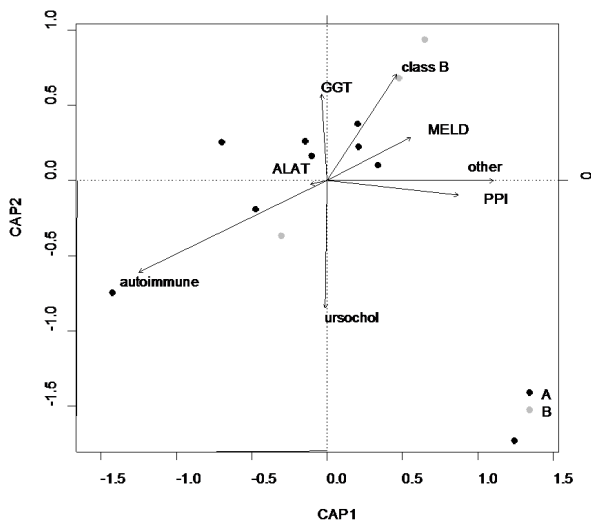

B

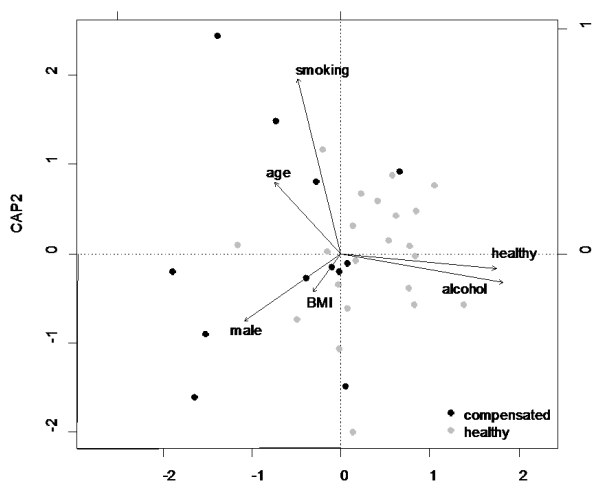

D

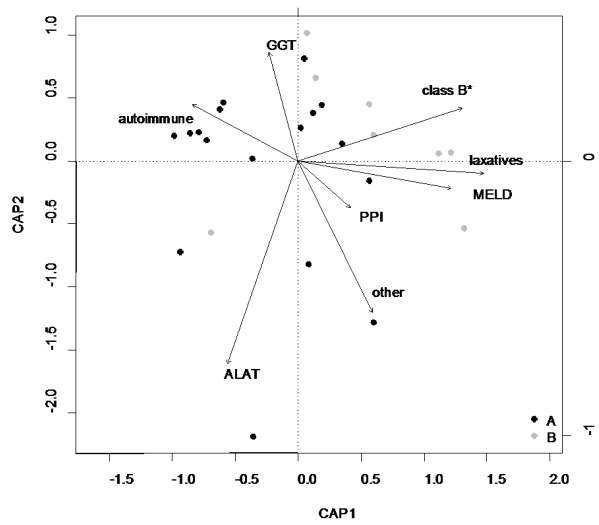

F

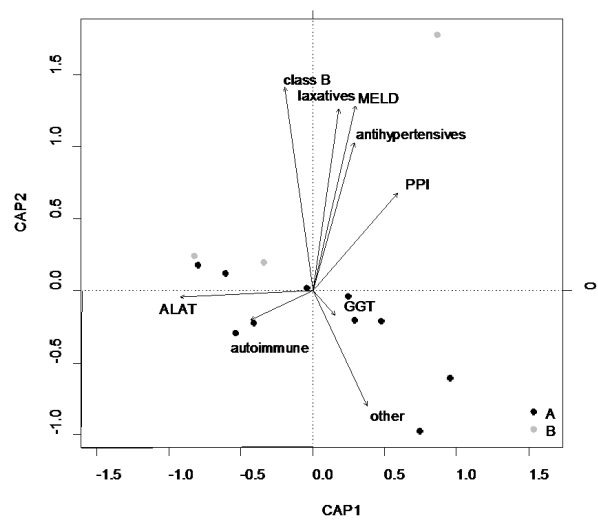

Figure 5.5 Distance based redundancy analysis (db-RDA) ordination on Bray-Curtis dissimilarity of fecal (a, d), sigmoid (b, e) and duodenum (c, f) from patients with compensated cirrhosis (CLC) and samples from healthy controls ( $a, b$ and $c$ ) or patients with compensated cirrhosis only ( $d$, e and $\mathrm{f}$ ). The vectors indicates the effect of each variable on the microbiota composition $(* P<0.05)$. 


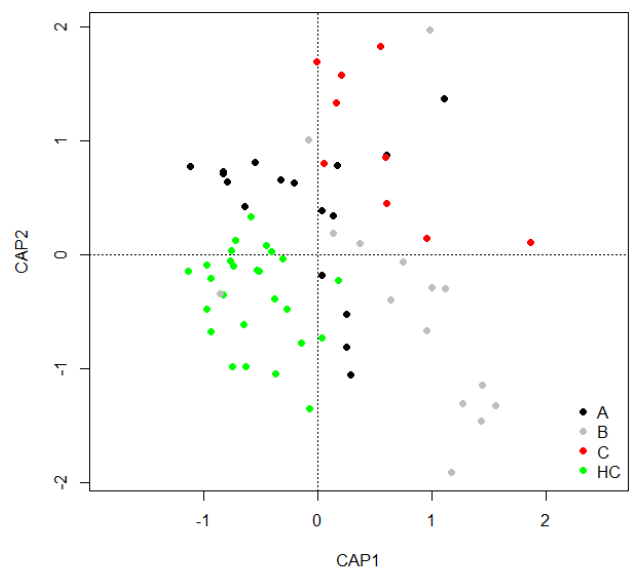

Figure 5.6 Distance based redundancy analysis (db-RDA) ordination based on Bray-Curtis dissimilarity of samples from $\mathrm{HC}$ and patients with compensated and decompensated liver cirrhosis (CLC) patients based on disease severity (Child-Pugh class A, B and C).

\section{Prediction of disease severity based on the fecal microbiota}

PLS-DA was performed to investigate whether the fecal microbiota could be used as a marker to predict disease severity. The model based on all phyla correctly classified $71 \%, 60 \%$ and $60 \%$ of the samples belonging to class A, class B and class C, respectively. Using a subset of peaks with a VIP score $>1$ or $>2.3$ did not improve the disease severity classification.

\section{Mucosal microbial community structure}

PCoA of the sigmoid biopsies did not show clustering based on health status (data not shown). Also no significant differences were found when comparing within-group BrayCurtis dissimilarity of CLC patients [0.62 (0.46-0.86)] and within-group Bray-Curtis dissimilarities of $\mathrm{HC}[0.58(0.38-0.96)]$. db-RDA of the Bray-Curtis dissimilarity showed no separation of sigmoid biopsies by health status $(P=0.076)$, age $(P=0.384)$, sex $(P=0.380)$, BMI $(P=0.608)$, smoking $(P=0.093)$ or alcohol use $(P=0.130)$ (Figure 5.5B). Furthermore, db-RDA showed no significant effect of disease severity $(P=0.709)$ and drug therapy ( $P=0.260$ for PPIs, $P=0.710$ for laxatives) on the microbiota composition of the sigmoid (Figure 5.5E).

Duodenal biopsies of CLC patients seemed to cluster together according to the PCoA of Bray-Curtis dissimilarities (supplemental data Figure S5.2). The within-group BrayCurtis dissimilarity was significantly higher for HC $[0.72(0.36-0.95)]$ than for liver cirrhotic patients $[0.56(0.38-0.79)]$, indicating that inter-individual variations in the microbiota composition of patients with CLC was significantly smaller as compared to that of $\mathrm{HC}(P=0.01)$. db-RDA demonstrated that the duodenal biopsies were separated by health status $(P=0.04)$ (Figure $5.5 C)$, but not by age $(P=0.713)$, sex $(P=0.051)$, BMI 
$(P=0.512)$, smoking $(P=0.755)$ or alcohol use $(P=0.127)$. db-RDA showed also no significant effect of disease severity $(P=0.135)$, etiology $(P=0.619)$ or drug therapy ( $P=0.300$ for PPIs, $P=0.211$ for laxatives) on the microbiota composition of the duodenum (Figure 5.5F).

\section{Bacterial taxa associated with compensated liver cirrhosis}

The abundance of six and 12 peaks were found to be significantly different (FDR cut off value $\leq 0.15$ ) between CLC patients and HC in fecal samples and duodenal biopsies, respectively (Table 5.2). An increase of Streptococcus bovis/gallolyticus and Streptococcus mitis (corresponding to four peaks for which identification was possible) was found in the fecal samples of CLC when compared with HC. Enterococcus spp. Staphylococcus spp., Lactobacillus Johnsonii and Lactobacillus plantarum (corresponding to nine peaks for which identification was possible) were found in higher abundance in the duodenal biopsies of CLC patients than in $\mathrm{HC}$.

As we observed microbial differences in fecal and duodenal samples, the fecal and duodenal peaks that significantly differed between patients with CLC and HC, were related to sugar ratios indicating large and small intestinal permeability, respectively. $A$ significant positive correlation was found between the $L / R$ ratio and the duodenal peaks 264_Firmicutes $(P=0.03, r=0.43)$ and 265_Firmicutes $(P=0.02, r=0.51)$, which both correspond to Lactobacillus johnsonii. No significant correlation was found between the S/E ratio and the six peaks in fecal samples, which significantly differed between CLC patients and $\mathrm{HC}$.

Table 5.2 Identification of species (feces).

\begin{tabular}{|c|c|c|c|c|c|c|}
\hline & Peak_ID & Bacterial species & $P$ value & DR value & $\begin{array}{c}\text { Mean } \\
\text { frequency } \\
\text { HC }\end{array}$ & $\begin{array}{c}\text { Mean } \\
\text { frequency } \\
\text { CLC }\end{array}$ \\
\hline \multirow[t]{6}{*}{ Feces } & 321_Firmicutes & Streptococcus bovis/gallolyticus & 0,00 & 0,02 & 3,85 & 9,36 \\
\hline & 322_Firmicutes & & 0,00 & 0,02 & 3,62 & 8,04 \\
\hline & 298_Firmicutes & Streptococcus mitis group & 0,00 & 0,02 & 1,54 & 5,68 \\
\hline & 299_Firmicutes & & 0,00 & 0,02 & 1,12 & 4,00 \\
\hline & 308_Bacteroidetes & Unclassified Bacteroidetes & 0,00 & 0,02 & 10,85 & 5,28 \\
\hline & 306_Bacteroidetes & & 0,00 & 0,02 & 9,15 & 4,20 \\
\hline \multirow[t]{13}{*}{ Duodenum } & 264_Firmicutes & Lactobacillus johnsonii & 0,00 & 0,15 & 0,50 & 3,23 \\
\hline & 265_Firmicutes & & 0,00 & 0,16 & 0,30 & 3,46 \\
\hline & 496_Firmicutes & & 0,00 & 0,15 & 0,25 & 2,23 \\
\hline & 495_Firmicutes & & 0,00 & 0,15 & 0,15 & 2,31 \\
\hline & 497_Firmicutes & & 0,01 & 0,15 & 0,35 & 1,77 \\
\hline & 276_Firmicutes & Enterococcus spp. & 0,00 & 0,14 & 1,85 & 4,77 \\
\hline & 485_Firmicutes & Lactobacillus plantarum & 0,00 & 0,15 & 0,45 & 2,23 \\
\hline & 292_Firmicutes & Staphylococcus spp. & 0,00 & 0,15 & 4,70 & 9,31 \\
\hline & 289_Firmicutes & & 0,00 & 0,15 & 2,75 & 6,46 \\
\hline & 366_Bacteroidetes & Unclassified Bacteroidetes & 0,00 & 0,15 & 0,35 & 2,69 \\
\hline & 368_Bacteroidetes & & 0,00 & 0,15 & 0,85 & 4,92 \\
\hline & 369_Bacteroidetes & & 0,00 & 0,15 & 0,75 & 3,77 \\
\hline & 367_Bacteroidetes & & 0,00 & 0,16 & 0,50 & 3,31 \\
\hline
\end{tabular}

$\mathrm{HC}$, healthy controls; CLC, compensated liver cirrhosis. 


\section{Discussion}

The present study showed that both fecal and duodenal microbiota composition of patients with compensated liver cirrhosis of various etiologies differed from healthy controls, as shown by an increased fecal and duodenal F/B ratio in these patients, and alterations in richness and diversity of the phyla Firmicutes and Bacteroidetes. The differences in fecal microbiota were even more pronounced with progression of liver cirrhosis, i.e. in patients with decompensated cirrhosis. Furthermore, patients with (de)compensated cirrhosis were found to cluster separately from HC (with regard to the fecal microbial community structure), and showed distinct clusters based on the Child-Pugh classification. No alterations were found in the microbiota composition of the sigmoid.

In the present study, we included a heterogeneous group of patients with compensated and decompensated cirrhosis, as present in daily clinical practice, and compared the results with age, sex, and BMI-matched controls. In addition, duodenal and sigmoid biopsies were available for subgroups. The microbiota composition was investigated by IS-pro, which distinguishes and identifies bacteria by phylum-specific primers combined with the length of the 16S-23S IS region. This technique is easy to implement in clinical practice and enables analysis of individual samples with a turnaround-time from sample delivery to ready-to-interpret results in less than 5 hours.

Significant differences were found in the fecal microbiota composition of compensated cirrhotic patients compared to age, sex and BMI-matched $\mathrm{HC}$, resulting in an increased $\mathrm{F} / \mathrm{B}$ ratio in the patients, which is in line with others. ${ }^{9-11}$ Total microbial richness and diversity in fecal samples was not affected, whereas both richness and diversity of the Firmicutes were significantly higher and richness of the Bacteroidetes was significantly lower in the patients when compared with controls. Furthermore, we found clustering of the subjects by health status on PCoA, and showed that the fecal microbiota composition of $\mathrm{HC}$ was more homogenous than that of patients with compensated cirrhosis. The above findings indicate that cirrhotic patients have a different fecal microbiota profile than $\mathrm{HC}$, which seems to take various forms.

The observed microbial changes in patients with compensated cirrhosis may be associated with host characteristics or etiological factors, such as alcohol use ${ }^{22}$ and obesity. ${ }^{4}$ However, we did not observe any effect of alcohol use, etiology, nor of BMI and drug therapy, although it should be noted that subgroups were rather small. Since only one patient with compensated cirrhosis used antibiotics within 2 months prior to this study, it is highly unlikely that this affected our findings. Based on the findings, the microbial changes seem to be associated with compensated cirrhosis itself, for example, by alterations in bile acid synthesis. ${ }^{23}$ Interestingly, the severity of the disease (by Child-Pugh class) was found to be associated with the microbiota composition, with more pronounced differences in Child-Pugh B versus A patients when compared to 
controls. This effect was further supported by the finding that the observed differences were even more pronounced with progression of the disease, i.e. in the patients with Child-Pugh C / decompensated cirrhosis. It should however be noted that the perturbations in the decompensated group might (at least in part) be affected by the high percentage antibiotic users in this group ( $n=10 / 16,63 \%)$.

The above findings are in line with others showing correlations between microbiota and disease severity, but few investigated the potential use of the microbiota to predict disease severity of liver cirrhosis. ${ }^{10,22,24}$ Using PLS-DA, we found that the fecal microbiota can be used to predict disease severity measured by the Child-Pugh classification. The performance of the model however differed based on the phyla used, and showed the best prediction when all phyla were combined together in one model (i.e. Firmicutes, Bacteroidetes and Proteobacteria). Although the prediction rate is modest, the results are promising considering the variation in drug therapy, etiology, and relatively small numbers, and warrants further studies.

Four out of six peaks of which the abundance in fecal samples differed significantly between controls and compensated cirrhotic patients, corresponded to known bacterial taxa. Most of them corresponded to Streptococcus spp., which include oral commensals (i.e. S. mitis). ${ }^{25}$ Oral bacteria were also found by others to be increased in fecal samples of cirrhotic patients. ${ }^{9,13}$ This may (in part) be caused by reduced bile acid synthesis, portal hypertension and/or altered motility. ${ }^{26,27,28,29}$

As data on the mucosa-associated microbiota in patients with cirrhosis are limited, we also characterized the microbiota in sigmoid and duodenal biopsies obtained from a subgroup of patients with compensated cirrhosis and controls.

In contrast to Bajaj et Il $^{16}$ no alterations were observed in the microbiota composition of the sigmoid between compensated cirrhotics and controls. In duodenal biopsies, the F/B ratio was significantly increased in patients with compensated cirrhosis versus HC, in line with findings of the fecal microbiota. Furthermore, microbial richness of Firmicutes was found to be significantly higher in duodenum of compensated cirrhotic patients than HC. Interestingly, these alterations resulted in a significantly more homogeneous duodenal microbiota composition in the compensated patient group, and were not affected by demographics, severity of disease, etiology nor drug therapy. These findings suggest that the compositional changes in the duodenum were most likely due to the cirrhosis and point to involvement of the small intestine in its etiology. Data from our group and from previous studies indicate that permeability of the small and/or large intestine is increased in cirrhotic patients. ${ }^{19,30}$ Changes in the intestinal microbiota together with alterations in permeability, may contribute to bacterial translocation and disease progression. A significant positive correlation was found between $L$. johnsonii and the L/R ratio, indicating small intestinal permeability. No further correlations were found with the S/E ratios (colonic permeability). The role of L. johnsonii in small intestinal permeability remains elusive as two studies showed the 
bacterium to decrease intestinal permeability and bacterial translocation, ${ }^{31,32}$ whereas another study contradicts these observations. ${ }^{33}$

To our knowledge, this is the first study that characterized the composition of the duodenal microbiota in patients with cirrhosis, and therefore the significance of these findings should be further investigated and confirmed. Despite the heterogeneity and small numbers of biopsies available, we did observe pronounced alterations in both the duodenum and feces. However, it should be noted that also the functional activity of the microbiota should be addressed in future studies to further unravel its role in the pathophysiology of liver cirrhosis.

In conclusion, this study showed the potency of IS-profiling to assess microbial changes in line with sequencing data. The observed gradient between Child-Pugh A, B and C cirrhosis shows that this technique has potential as marker to monitor disease progression, which is supported by the PLS-DA results. Furthermore, we found clear indications for involvement of the duodenal microbiota in liver cirrhosis and possible associations with small intestinal permeability, which warrant further evaluation. 


\section{References}

1. Eckburg PB, Bik EM, Bernstein CN, et al. Diversity of the human intestinal microbial flora. Science 2005;308:1635-8.

2. Zoetendal EG, Rajilic-Stojanovic M, de Vos WM. High-throughput diversity and functionality analysis of the gastrointestinal tract microbiota. Gut 2008;57:1605-15.

3. Willing BP, Dicksved J, Halfvarson J, et al. A pyrosequencing study in twins shows that gastrointestinal microbial profiles vary with inflammatory bowel disease phenotypes. Gastroenterology 2010;139:1844-54 e1.

4. Turnbaugh PJ, Hamady M, Yatsunenko T, et al. A core gut microbiome in obese and lean twins. Nature 2009;457:480-4.

5. Qin J, Li Y, Cai Z, et al. A metagenome-wide association study of gut microbiota in type 2 diabetes. Nature 2012;490:55-60.

6. Mouzaki M, Comelli EM, Arendt BM, et al. Intestinal microbiota in patients with nonalcoholic fatty liver disease. Hepatology 2013;58:120-7.

7. Mutlu EA, Gillevet PM, Rangwala $\mathrm{H}$, et al. Colonic microbiome is altered in alcoholism. American journal of physiology Gastrointestinal and liver physiology 2012;302:G966-78.

8. Garcia-Tsao G, Friedman S, Iredale J, Pinzani M. Now there are many (stages) where before there was one: In search of a pathophysiological classification of cirrhosis. Hepatology 2010;51:1445-9.

9. Qin N, Yang F, Li A, et al. Alterations of the human gut microbiome in liver cirrhosis. Nature 2014;513:59-64.

10. Wei X, Yan X, Zou D, et al. Abnormal fecal microbiota community and functions in patients with hepatitis $B$ liver cirrhosis as revealed by a metagenomic approach. BMC Gastroenterol 2013;13:175.

11. Chen Y, Yang F, Lu H, et al. Characterization of fecal microbial communities in patients with liver cirrhosis. Hepatology 2011;54:562-72.

12. Bajaj JS, Ridlon JM, Hylemon PB, et al. Linkage of gut microbiome with cognition in hepatic encephalopathy. Am J Physiol Gastrointest Liver Physiol 2012;302:G168-75.

13. Zhang Z, Zhai H, Geng J, et al. Large-scale survey of gut microbiota associated with MHE Via 16S rRNAbased pyrosequencing. Am J Gastroenterol 2013;108:1601-11.

14. Kakiyama G, Pandak WM, Gillevet PM, et al. Modulation of the fecal bile acid profile by gut microbiota in cirrhosis. J Hepatol 2013;58:949-55.

15. WHO Global Infobase: data on overweight and obesity, mean BMI, healthy diets and physical inactivity at http://www.who.int/mediacentre/factsheets/fs311/en/index.html.)

16. Bajaj JS, Hylemon PB, Ridlon JM, et al. Colonic mucosal microbiome differs from stool microbiome in cirrhosis and hepatic encephalopathy and is linked to cognition and inflammation. Am J Physiol Gastrointest Liver Physiol 2012;303:G675-85.

17. Bajaj JS, Heuman DM, Hylemon PB, et al. Altered profile of human gut microbiome is associated with cirrhosis and its complications. J Hepatol 2014;60:940-7.

18. Budding $A E$, Grasman $M E$, Lin $F$, et al. IS-pro: high-throughput molecular fingerprinting of the intestinal microbiota. FASEB J 2010;24:4556-64.

19. Pijls KE, Koek GH, Elamin EE, de Vries $H$, Masclee AA, Jonkers DM. Large intestine permeability is increased in patients with compensated liver cirrhosis. Am J Physiol Gastrointest Liver Physiol 2014;306:G147-53.

20. Pugh RN, Murray-Lyon IM, Dawson JL, Pietroni MC, Williams R. Transection of the oesophagus for bleeding oesophageal varices. Br J Surg 1973;60:646-9.

21. de Meij TG, Budding AE, de Groot EF, et al. Composition and stability of intestinal microbiota of healthy children within a Dutch population. FASEB J 2015.

22. Chen Y, Qin N, Guo J, et al. Functional gene arrays-based analysis of fecal microbiomes in patients with liver cirrhosis. BMC Genomics 2014;15:753.

23. Ridlon JM, Alves JM, Hylemon PB, Bajaj JS. Cirrhosis, bile acids and gut microbiota: unraveling a complex relationship. Gut microbes 2013;4:382-7.

24. Steed H, Macfarlane GT, Blackett KL, et al. Bacterial translocation in cirrhosis is not caused by an abnormal small bowel gut microbiota. FEMS Immunol Med Microbiol 2011;63:346-54. 
25. Mitchell J. Streptococcus mitis: walking the line between commensalism and pathogenesis. Molecular oral microbiology 2011;26:89-98.

26. Lakshmi CP, Ghoshal UC, Kumar S, et al. Frequency and factors associated with small intestinal bacterial overgrowth in patients with cirrhosis of the liver and extra hepatic portal venous obstruction. Dig Dis Sci 2010;55:1142-8.

27. Gunnarsdottir SA, Sadik R, Shev S, et al. Small intestinal motility disturbances and bacterial overgrowth in patients with liver cirrhosis and portal hypertension. Am J Gastroenterol 2003;98:1362-70.

28. Merritt ME, Donaldson JR. Effect of bile salts on the DNA and membrane integrity of enteric bacteria. J Med Microbiol 2009;58:1533-41.

29. Bouhnik Y, Alain S, Attar A, et al. Bacterial populations contaminating the upper gut in patients with small intestinal bacterial overgrowth syndrome. Am J Gastroenterol 1999;94:1327-31.

30. Pijls KE, Jonkers DM, Elamin EE, Masclee AA, Koek GH. Intestinal epithelial barrier function in liver cirrhosis: an extensive review of the literature. Liver international : official journal of the International Association for the Study of the Liver 2013;33:1457-69.

31. Chiva M, Soriano G, Rochat I, et al. Effect of Lactobacillus johnsonii La1 and antioxidants on intestinal flora and bacterial translocation in rats with experimental cirrhosis. J Hepatol 2002;37:456-62.

32. Southcott E, Tooley KL, Howarth GS, Davidson GP, Butler RN. Yoghurts containing probiotics reduce disruption of the small intestinal barrier in methotrexate-treated rats. Dig Dis Sci 2008;53:1837-41.

33. Eutamene $\mathrm{H}$, Lamine $\mathrm{F}$, Chabo $\mathrm{C}$, et al. Synergy between Lactobacillus paracasei and its bacterial products to counteract stress-induced gut permeability and sensitivity increase in rats. J Nutr 2007;137:1901-7. 


\section{Supplemental data}

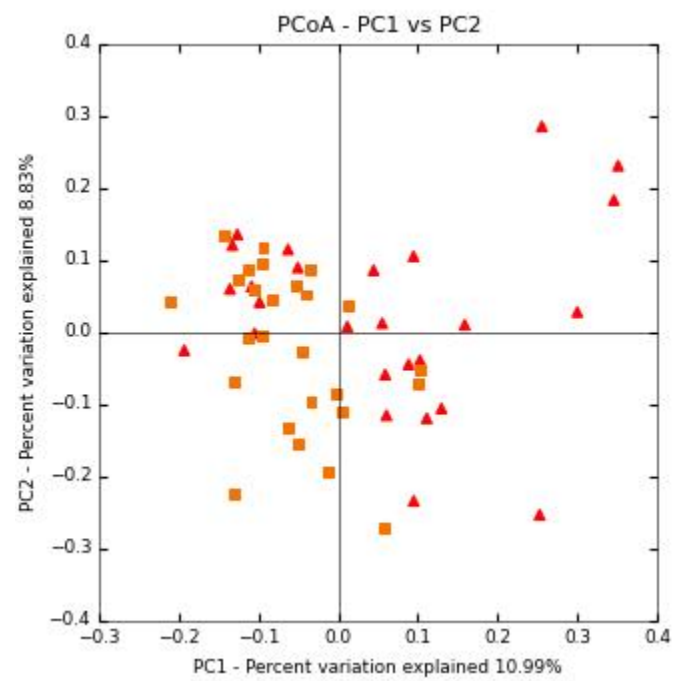

Figure S5.1 Principal coordinate analysis (PCoA) on Bray Curtis dissimilarity of fecal samples between healthy controls (orange squares) and patients with compensated cirrhosis (red triangles) based on health status.

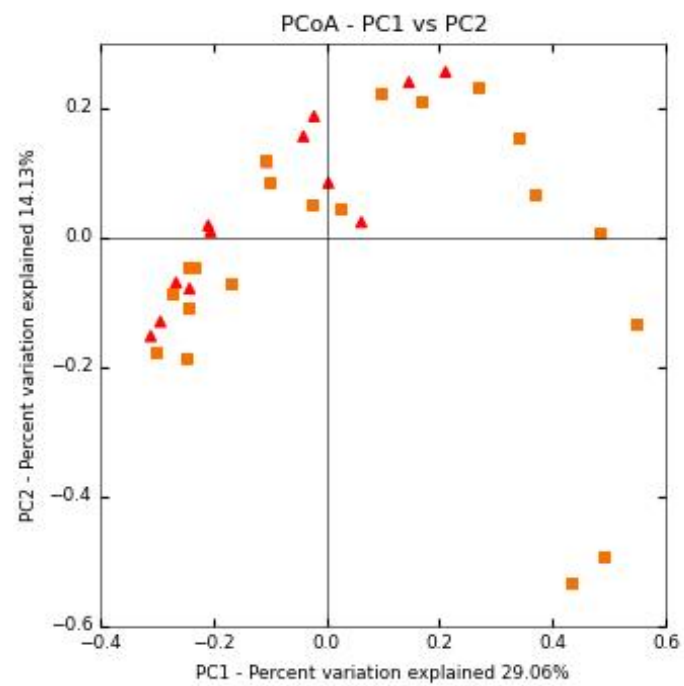

Figure S5.2 Principal coordinate analysis (PCoA) on Bray Curtis dissimilarity of duodenal samples between healthy controls (orange squares) and patients with compensated cirrhosis (red triangles) based on health status. 


\section{Chapter 6}

The role of the intestine in animal models with dietinduced hepatic steatosis and steatohepatitis

K.E. Pijls*

B. van de Wier*

D.M.A.E. Jonkers

F. Attema

E. Villamor

M. Elizalde

A.A.M. Masclee

G.R. Haenen

A. Bast

G.H. Koek

*Both authors contributed equally to this work

Submitted 


\section{Abstract}

Non-alcoholic fatty liver disease (NAFLD) is a disorder ranging from hepatic steatosis to hepatic steatosis combined with inflammation [non-alcoholic steatohepatitis (NASH)], which can progress to fibrosis and cirrhosis. Factors that trigger hepatic inflammation remain largely unknown, but increasing evidence indicates that the intestine may be involved. We aimed to investigate the role of the intestine in the onset of hepatic inflammation in non-alcoholic fatty liver disease (NAFLD) using animal models.

Therefore, the intestinal epithelial barrier function, oxidative stress and motility of female wild type C57BI/6J (WT) mice were compared with that of female low density lipoprotein knock-out $\left(L d l r^{-1}\right)$ mice on a high-fat and high-cholesterol diet for 3 weeks.

Hepatic steatosis was comparable in both mouse models, while inflammation was only demonstrated in $\mathrm{Ldll}^{-/}$mice. No alterations of the intestinal epithelial barrier function in ileum and sigmoid were found between WT and $L d l r^{-/}$mice. Furthermore, no differences were found in the transcription of oxidative stress-related genes, apart from an upregulation of GCLM and $\mathrm{XDH}$ in the sigmoid of $L d l r^{--}$mice. The circular smooth muscle contractility of duodenal segments was also not different between both mouse models.

These findings show that no major differences were found in epithelial barrier function, oxidative stress and motility of both the small and large intestine between WT and Ldlr ${ }^{-/}$mice after a 3-week period of high-fat and high-cholesterol feeding. This indicates that the intestine does not play a major role in the onset of hepatic inflammation in these NAFLD mouse models. 


\section{Introduction}

Non-Alcoholic Fatty Liver Disease (NAFLD) is the most common chronic liver disease with a prevalence rate of about $10-35 \%$ in the general population and is associated with metabolic risk factors such as obesity and type II diabetes. ${ }^{1,2}$ NAFLD can progress from simple hepatic steatosis to steatohepatitis [i.e. non-alcoholic steatohepatitis (NASH)], which is characterized by hepatic steatosis, inflammation and hepatocellular injury with or without fibrosis. ${ }^{3}$ Although hepatic steatosis is largely considered benign, $\mathrm{NASH}$ is associated with the development of cirrhosis, liver failure and hepatocellular carcinoma. Knowledge of factors triggering the transition from steatosis to NASH is therefore of high clinical relevance, but until now is incompletely understood.

In addition to insulin resistance and hepatic oxidative stress, increasing evidence indicates that also the intestine may be involved in the progression towards NASH. In mouse models, induction of intestinal inflammation by dextran sulfate sodium was found to increase intestinal permeability, endotoxin levels, hepatic inflammation and fibrosis. ${ }^{4,5}$ Intestinal epithelial barrier dysfunction facilitates translocation of bacteria and their products (e.g. endotoxin) into the portal circulation, which can activate hepatic Kupffer cells, subsequently leading to the production of inflammatory mediators and the induction of inflammation and fibrosis in the liver. ${ }^{6}$

Epithelial barrier function is regulated by intercellular tight junctions (TJ) and adherens junctions (AJ), which can be affected, for example, by high-fat intake, ${ }^{7}$ bacteria, ${ }^{8}$ cytokines $^{9}$ and oxidative stress. ${ }^{10}$ An increased intestinal permeability has been found in some studies with NAFLD patients, ${ }^{11,12}$ but not in all. ${ }^{13,14}$ Animal studies have shown evidence for epithelial barrier dysfunction in mice on a high fructose diet that developed hepatic steatosis with also some signs of inflammation. ${ }^{15-18}$ Furthermore, significantly higher serum endotoxin levels were found in rats with NASH induced by high-fat feeding compared to rats with a normal diet, which was accompanied by oxidative stress in the small intestine [i.e. reduced levels of superoxide dismutase (SOD) and increased levels of malondialdehyde (MDA)]. ${ }^{19}$ Oxidative stress has been found to affect TJ integrity and thereby can contribute to increased intestinal permeability. $^{10,20}$

In addition, an altered intestinal microbiota composition can promote barrier disruption and bacterial translocation. Recently, the fecal microbiota composition with respect to Bacteroidetes was found to be significantly different in patients with NASH compared to those with simple hepatic steatosis and healthy controls. ${ }^{21}$ Small intestinal bacterial overgrowth has been reported in patients and animals with $\operatorname{NAFLD}^{11,13,22,23}$ and may be related to intestinal dysmotility. However, data on intestinal motility in NAFLD are limited and inconsistent. ${ }^{12,13,23-25}$

Animal models suggest that alterations in the intestine may occur in NAFLD, but studies comparing the role of the intestine between hepatic steatosis and NASH are scarce and mainly focus on the proximal small intestine. We hypothesize that the 
presence of small and large intestinal alterations are more frequent in 'early' NASH compared to steatosis.

Aim of the present study was to explore the role of the intestine in the transition of hepatic steatosis to steatohepatitis using two mouse models known to develop either hepatic steatosis or hepatic steatosis with inflammation after a short term feeding with a high-fat and high-cholesterol diet. ${ }^{26}$ We investigated epithelial barrier function, oxidative stress and motility in both the small and large intestine.

\section{Materials and methods}

\section{Animals and treatment}

Placebo-treated female C57BI/6J wild type mice (WT mice) and female low density lipoprotein (LDL) receptor knock-out $\left(\mathrm{Ldll}^{-/}\right)$mice on a C57BI/6J background (Jackson Laboratories) used in a prior study investigating the effect of the flavonoid monoHER on oxidative stress and the adaptive response in the liver during the onset of NAFLD were available for analyses of the intestine. The design of the study has been described in detail elsewhere. ${ }^{27}$

Briefly, mice were housed in groups of two per cage under standard conditions and received standard chow and tap water ad libitum. At the age of 13 weeks, all mice received a high-fat and high-cholesterol diet (HFD) containing fat derived from palm oil and $0.2 \%$ cholesterol (Research Diets, New Brunswick, USA, D11012302). Placebotreated WT $(n=12)$ and $\mathrm{Ldll}^{-/-}$mice $(n=12)$ received a daily subcutaneous injection of a $25 \mu \mathrm{l} / \mathrm{g}$ physiological saline solution $(0.9 \% \mathrm{NaCl})$ as placebo intervention. Diet and placebo treatment were continued for 3 weeks. Sacrificing of the mice and tissue isolation of liver were performed as described previously. ${ }^{27}$ The whole intestine was removed and divided into three segments, proximal small intestine (i.e. duodenum), distal small intestine (i.e. ileum) and distal large intestine (i.e. sigmoid) for further investigation.

Experiments were performed according to Dutch laws, approved by the Committee for Animal Welfare of Maastricht University (DEC2010-175).

\section{Liver}

\section{Histology}

Four $\mu \mathrm{m}$ formalin-fixed paraffin embedded liver sections were stained with hematoxylin and eosin. They were analyzed to quantify steatosis $(<5 \%=0 ; 5-33 \%=1$; $>33-66 \%=2 ;>66 \%=3$ ) and inflammation (no foci=0; $<2$ foci/field=1; $2-4$ foci/field $=2 ;>4$ foci/field=3) in line with the NAFLD activity score (NAS) by two blinded experienced investigators. ${ }^{28}$ Foci of inflammation were counted in 4 high power fields using 200 magnification. 
Infiltrated macrophages and neutrophils were evaluated by immunohistochemical staining in frozen liver sections $(5 \mu \mathrm{m})$ using macrophage marker Mac-1 and neutrophil marker NIMP. ${ }^{29}$ Pictures were taken with a Nikon digital camera DMX1200 and ACT-1 v2.63 software. Cells were counted in 6 photographs of each liver section by two independent investigators and were expressed as cells $/ \mathrm{mm}^{2}$.

\section{Intestine}

\section{Paracellular permeability}

Immediately after dissection, two segments of the ileum and one segment of the sigmoid were placed in freshly-prepared ice-cold Krebs-Ringer bicarbonate buffer (KRB) [composition in $\mathrm{mM}: \mathrm{NaCl} 115.9, \mathrm{KCl} 3.6, \mathrm{MgSO}_{4} 1.2, \mathrm{CaCl}_{2} 1.25, \mathrm{KH}_{2} \mathrm{PO} 4$ 1.4, $\mathrm{NaHCO}_{3}$ 23.1, Na-pyruvate 5.7, Na-glutamate 5.3, glucose 10 (serosa) and mannitol 10 (mucosa)]. Mesenteric attachments were removed and the segments were rinsed to remove intestinal contents and opened in a longitudinal direction. Whole-thickness segments were mounted in modified $1.5 \mathrm{ml}$ Ussing chambers (Harvard Apparatus Inc., Holliston, Mass., USA) with an opening of $9 \mathrm{~mm}$ in diameter and an exposed tissue area of $1.76 \mathrm{~mm}^{2}$, as described by Wallon et al. ${ }^{30,31}$ After mounting, both the mucosal and serosal compartments were filled with $1.5 \mathrm{ml} \mathrm{KRB} \mathrm{(pH} \mathrm{7.4).} \mathrm{This} \mathrm{solution} \mathrm{was}$ continuously oxygenated, stirred by gas flow in the chambers and kept at $37^{\circ} \mathrm{C}$ with a heater block system during the total experimental period. After a 40 min equilibration period, the KRB in the mucosal compartment was replaced with KRB containing horseradish peroxidase (HRP) at a final concentration of $10 \mu \mathrm{M}$ and fluorescein isothiocyanate (FITC)-labeled dextran of $4 \mathrm{kDa}$ (FD4) at a final concentration of $100 \mu \mathrm{M}$ (Sigma Aldrich, St. Louis, USA) to evaluate (paracellular) permeability. At the same time, KRB in the serosal compartment was replaced with fresh KRB. The potential difference (PD), transmucosal electrical resistance (TER) and short-circuit current (ISC) were followed for $120 \mathrm{~min}$. Experiments were done in open-circuit conditions with assessment of electrophysical parameters at $2 \mathrm{~min}$ intervals. Samples from the serosal compartments were taken after $60 \mathrm{~min}$ (and replaced with fresh KRB) and $120 \mathrm{~min}$ and stored at $-80^{\circ} \mathrm{C}$ until further analysis of HRP and FD4.

HRP in samples was determined using the QuantaBlu ${ }^{\mathrm{TM}}$ Fluorogenic Peroxidase Substrate Kit (Pierce, Rockford, Illinois, USA) according to the manufacturer's instructions. HRP was expressed as pmol/ml. FD4 in samples was determined spectrophotometrically by measuring the fluorescence intensity at $525 \mathrm{~nm}$ by the SpectraMax M2 MultiMode Microplate Reader (Molecular Devices, LLC, USA) and expressed as $\mathrm{pmol} / \mathrm{ml}$.

\section{RNA isolation and quantitative Real-Time Polymerase Chain Reaction (qRT-PCR)}

Ileum and sigmoid segments for gene transcription were rinsed with PBS to remove intestinal contents, opened in a longitudinal direction and then directly snap-frozen in 
liquid nitrogen and stored at $-80^{\circ} \mathrm{C}$ until further analyses. Transcription of genes involved in intestinal barrier function [i.e. occludin, claudin-3, claudin-4, zonula occludens-1 (ZO-1) and myosin light-chain kinase (MLCK)] and in oxidative stress [i.e. glutamate-cysteine ligase, catalytic subunit (GCLC), glutamate-cysteine ligase, modifier subunit (GCLM), glutathione synthetase (GS), glutathione peroxidases (GPX1, GPX2 and GPX3), glutathione reductase $(G R)$, and xanthine dehydrogenase $(X D H)$ ] were determined by qRT-PCR.

Total RNA was isolated from the frozen segments using TRIzol reagent (Invitrogen, Carlsbad, USA) and purified with the RNeasy Mini Kit (Qiagen, Venlo, the Netherlands). The concentration of purified RNA was measured with the NanoDrop. Finally, $500 \mathrm{ng}$ total RNA was used as a template for the cDNA reaction, which was synthesized using the iScript cDNA Synthesis Kit (Bio-Rad, Veenendaal, the Netherlands). The cDNA was diluted to a concentration of $4 \mathrm{ng} / \mu \mathrm{L}$. Each reaction contained $5 \mu \mathrm{L}$ cDNA template solution, $12.5 \mu \mathrm{L}$ iQ SYBR Green Supermix (Bio-Rad), $1 \mu \mathrm{L}$ forward and reverse primers $(10 \mu \mathrm{M})$ and $5.5 \mu \mathrm{L}$ sterile water. Primer sequences have been listed in Table 6.1. Housekeeping genes included were 18S rRNA and glyceraldehyde-3-phosphate dehydrogenase (GAPDH). Reactions were run on the My IQ Single Color RT-PCR Detection System (Bio-Rad). PCR conditions used were $3 \mathrm{~min}$ at $95^{\circ} \mathrm{C}$, followed by 40 amplification cycles of $10 \mathrm{sec}$ at $95^{\circ} \mathrm{C}$ and $45 \mathrm{sec}$ at $60^{\circ} \mathrm{C}$. Data were presented as normalized expression ratios.

Table 6.1 Primer sequences of housekeeping genes and genes associated with intestinal barrier function and oxidative stress.

\begin{tabular}{llll}
\hline \multicolumn{2}{c}{ Sequence ID } & Forward primer & Reverse primer \\
\hline $\begin{array}{lll}\text { Housekeeping genes } \\
\text { 18S rRNA }\end{array}$ & NR_003278 & 5'-GTAACCCGTTGAACCCCATT-3' & \\
GAPDH & NR_008084.2 & 5'-TGCACCACCAACTGCTTAGC-3' & 5'-CCATCCAATCGGTAGTAGCG-3' \\
Genes associated with intestinal barrier function & \\
Occludin & NM_008756 & 5'-TCAGGGAATATCCACCTATCACTTCAG-3' & 5'-CATCAGCAGCAGCCATGTACTCTTCAC-3' \\
Claudin-3 & NM_009902.4 & 5'-CGACAAGTATGCACCCACCA-3' & 5'-CGTAGTCCTTGCGGTCGTAG-3' \\
Claudin-4 & NM_009903.2 & 5'-TGATTATGGTGCCCGTGTCC-3' & 5'-GAGTAGGGCTTGTCGTTGCTA-3' \\
ZO-1 & NM_009386.2 & 5'-GGCCCTAAACCTGTCCCTCA-3' & 5'-TATCTTCGGGTGGCTTCACT-3' \\
MLCK & NM_139300.3 & 5-GCGCTGCTAGATTTGACTGC-3' & 5'-TCTATCTGGAAGTGGCGGGA-3' \\
Genes associated with oxidative stress & \\
GCLC & NM_010295.2 & 5'-TGGAAGTTGATGTGGACACC-3' & 5'-GTCTTGCTTGTAGTCAGGATGG-3' \\
GCLM & NM_008129.4 & 5'-GGCACAGGTAAAACCCAATAGTAAC-3' & 5'-CAAATTGTTTAGCAAAGGCAGTCA-3' \\
GS & NM_008161.3 & 5'-GGTGACGTATGCCCCATTCA-3' & 5'-ACGGGCAGTATAGTCGTCCT-3' \\
GPX1 & NM_008160.6 & 5'-CCGACCCCAAGTACATCATT-3' & 5'-TATGTCAGGTTCGATGTCGATGG-3' \\
GPX2 & NM_030677.2 & 5'-TATGTCAGGTTCGATGTCGATGG-3' & 5'-CGGGTCATCATAAGGGTAGG-3' \\
GPX3 & NM_008161.3 & 5'-CCGGGGACAAGAGAAGTCTAA-3' & 5'-TGAGGGCTCCATACTCGTAGA-3' \\
GR & NM_010344.4 & 5'-CAGGGGATTGGCTGTGATGAGATGC-3' & 5'-GAGGTAGGATGAATGGCAACTGTG-3' \\
XDH & NM_011723.2 & 5'-GACCAGACGATTGCTCCCTC-3' & 5'-GGGGAGTGTCTTTCAGCCTC-3' \\
\hline
\end{tabular}

$18 \mathrm{~S}$ rRNA, 18S ribosomal RNA; GAPDH, glyceraldehyde-3-phosphate dehydrogenase; ZO-1, zonula occludens-1; MLCK, myosin light chain kinase; GCLC, glutamate-cysteine ligase, catalytic subunit; GCLM, glutamate-cysteine ligase, modifier subunit; GS, glutathione synthetase; GPX, glutathione peroxidase; GR, glutathione reductase; XDH, xanthine dehydrogenase. 


\section{Muscle contractility}

Two segments of duodenum from each mouse were freed from mesenteric attachments, rinsed with PBS to remove intestinal contents and cut into smaller segments. Whole-thickness segments ( $\sim 2.5 \mathrm{~mm}$ long) were suspended in the direction of circular smooth muscle fibers in thermostatically controlled $\left(37^{\circ} \mathrm{C}\right)$ organ baths $(5 \mathrm{ml}$ capacity) containing KRB buffer (composition in $\mathrm{mM}: \mathrm{NaCl} 117.5, \mathrm{KCl} 5.6, \mathrm{MgSO}_{4} 1.18$, $\mathrm{CaCl}_{2} 2.5, \mathrm{NaHPO}_{4} 1.28, \mathrm{NaHCO}_{3} 25$, and glucose 5.5) continuously oxygenated with $95 \% \mathrm{O}_{2}$ and $5 \% \mathrm{CO}_{2}(\mathrm{pH} 7.4)$. Contractile responses were measured with an isometric force-displacement transducer (model PRE 206-4, Cibertec, Madrid, Spain). The signal was amplified, A/D converted (PowerLab, ADInstruments Pty., Castle Hill, Australia), and recorded (Chart v5.2, ADInstruments Pty.). The intestinal rings were subjected to a tension of $9.8 \mathrm{mN}$ and allowed to equilibrate for $30 \mathrm{~min}$. During the equilibration, the bath solution was changed once at $15 \mathrm{~min}$ and the resting tension was readjusted to the $1 \mathrm{~g}$ level every $5 \mathrm{~min}$, except for the last $5 \mathrm{~min}^{32}$

After the equilibration period, spontaneous contractions were recorded during $3 \mathrm{~min}$ and thereafter a contraction was elicited by raising the $\mathrm{K}^{+}$concentration of the KRB buffer to $62.5 \mathrm{mM}$ in exchange for $\mathrm{Na}^{+}$. Afterwards, the tissues were washed three times with KRB buffer and allowed to equilibrate for another 20 minutes before performing a cumulative concentration-response curve to carbachol (10 nM - $10 \mu \mathrm{M})$. Then the tissues were washed again ( 3 times) and allowed to equilibrate for $20 \mathrm{~min}$ before performing a contraction with carbachol $(1 \mu \mathrm{M})$. When this contraction reached a steady state, a cumulative concentration-response curve to isoprenaline (10 nM - 1 $\mu \mathrm{M})$ was performed. At the end of the curve, tissues were maximally relaxed with papaverine $(0.1 \mathrm{mM})$. Length and mass (dry weight) of each segment were noted on completion of the experiments and cross sectional area (CSA) was determined for each tissue ring using the equation "CSA $\left(\mathrm{mm}^{2}\right)=$ mass $(\mathrm{mg})$ [length $(\mathrm{mm})$ density $\left(\mathrm{mg} \mathrm{mm} \mathrm{m}^{-3}\right]^{-1}$ ", where intestinal muscle density was assumed to be $1.05 \mathrm{mg} \mathrm{mm}^{-3}$. $^{33}$ The spontaneous contractions and the contractile responses to carbachol were assessed using the integrated mechanical activity (IMA) per second ( $\mathrm{mN} \mathrm{s}^{-1}$ ), normalized per CSA. The responses to $62.5 \mathrm{mM} \mathrm{K}^{+}$were assessed as peak values $(\mathrm{mN})$ and normalized per CSA. The relaxations evoked by isoprenaline in carbacholcontracted tissues were expressed as a percentage of the relaxation induced by 0.1 $\mathrm{mM}$ papaverine at the end of the experiment, which was arbitrarily defined as $100 \%$ relaxation.

\section{Statistical analysis}

Statistical analyses were performed using SPSS version 20.0. Data were tested for normality by the Kolmogorov-Smirnoff test. Normally distributed data were compared using an independent t-test. The Mann-Whitney $U$-test was used for non-parametric data. A $P$ value $<0.05$ was considered statistically significant using a 2-tailed test. 


\section{Results}

\section{Baseline characteristics}

Twelve WT and twelve $L d l r^{-/}$placebo-treated mice of a previous study ${ }^{27}$ were included for analysis in the current study. All mice completed the high-fat and high-cholesterol diet for 3 weeks. No differences were found with regard to food intake, body and liver weights and liver-to-body weight ratio between WT and $\mathrm{LdIr}^{-/}$mice (Supplemental data).

\section{Liver Histology}

No differences were found in the degree of steatosis between WT and $L d l r^{-/-}$mice, but the number of inflammatory foci per microscopic field was significantly higher in the Ldlr $^{-/-}$compared to the WT mice $(P<0.01)$.

Furthermore, the numbers of infiltrated macrophages and neutrophils were found to be significantly higher in the livers of $L d l r^{--}$mice compared to WT mice $(P<0.01)$ (manuscript in preparation).

\section{Intestinal epithelial barrier function}

Segments of ileum and sigmoid from 6 WT and $6 \mathrm{Ldlr}^{-/-}$mice were available for analysis of paracellular permeability in Ussing chamber experiments. The TER did not change significantly over time, indicating good tissue viability (data not shown). Furthermore, no significant differences in paracellular permeability to HRP and FD4 were observed in both ileum and sigmoid after $60 \mathrm{~min}$ between WT and Ldlr-/- mice (Table 6.2). Paracelullar permeability was also not significantly different after $120 \mathrm{~min}$ (data not shown).

Table 6.2 Paracellular permeability to FD4 and HRP in ileum and sigmoid after $60 \mathrm{~min}$.

\begin{tabular}{lccc}
\hline & WT $(\mathrm{n}=6)$ & $\operatorname{Ldll}^{-1 /}(\mathrm{n}=6)$ & $P$ value \\
\hline Ileum & & & \\
FD4 $(\mathrm{pmol} / \mathrm{ml})$ & $7934.10(6460.10-9442.50)$ & $7529.70(6618.00-8871.80)$ & 0.749 \\
HRP $(\mathrm{pmol} / \mathrm{ml})$ & $0.014(0.006-0.119)$ & $0.015(0.006-0.151)$ & 0.873 \\
Sigmoid & & & \\
FD4 $(\mathrm{pmol} / \mathrm{ml})$ & $7729.60(5600.50-8986.30)$ & $7263.90(5858.40-8220.80)$ & 0.337 \\
HRP $(\mathrm{pmol} / \mathrm{ml})$ & $0.006(0.005-0.387)$ & $0.009(0.006-0.040)$ & 0.262 \\
\hline
\end{tabular}

Values are presented as medians (range). FD4, fluorescein isothiocyanate-labeled dextran of 4 kDa; HRP, horseradish peroxidase.

As paracellular permeability is regulated by TJs, we also examined gene expression of key TJ proteins in segments of ileum and sigmoid from $12 \mathrm{WT}$ and $12 \mathrm{Ldll} \mathrm{r}^{-/}$mice by qRT-PCR. Gene transcription of claudin-3, claudin-4, occludin, ZO-1 and MLCK did not differ in ileum nor in sigmoid between both mouse models (Table 6.3). 
Table 6.3 Transcription of genes involved in intestinal barrier function

\begin{tabular}{lccc}
\hline & WT $(\mathrm{n}=12)$ & $\mathrm{Ldl}^{-/}(\mathrm{n}=12)$ & $P$ value \\
\hline Ileum & & & \\
Claudin 3 & $0.5349(0.0804-2.7076)$ & $0.4495(0.0798-1.9791)$ & 0.603 \\
Claudin 4 & $0.0026(0.0004-0.0190)$ & $0.0018(0.0003-0.0167)$ & 0.419 \\
Occludin & $0.3360(0.0488-1.5987)$ & $0.5412(0.0294-0.9907)$ & 0.773 \\
ZO-1 & $0.3989(0.0476-2.5724)$ & $0.3533(0.0482-1.0197)$ & 0.603 \\
MLCK & $0.4971(0.0720-2.8891)$ & $0.3673(0.0727-1.5448)$ & 0.488 \\
Sigmoid & & & \\
Claudin 3 & $0.9782(0.1915-4.6187)$ & $1.0886(0.4306-4.1264)$ & 0.564 \\
Claudin 4 & $0.5314(0.0440-4.9202)$ & $1.2884(0.1719-5.0948)$ & 0.106 \\
Occludin & $0.3142(0.1187-3.3108)$ & $0.7334(0.3370-5.0152)$ & 0.057 \\
ZO-1 & $0.3474(0.0363-2.2743)$ & $0.5057(0.1750-2.5918)$ & 0.386 \\
MLCK* & - & - & - \\
\hline
\end{tabular}

All data are normalized expression ratios, presented as medians (range). *MLCK was not included, because the majority of the values were below the detection level.

\section{Intestinal oxidative stress}

As the intestinal barrier can be disrupted by oxidative stress, the transcription of genes associated with oxidative stress was analyzed in ileum and sigmoid from 12 WT and 12 $\mathrm{Ldlr}^{-/}$mice. No significant differences were found for GCLC, GCLM, GS, GR, GPX1, GPX2, GPX3 and XDH between both mouse models in ileum (data not shown). In sigmoid, gene transcription of GCLM as well as XDH was significantly upregulated in Ldlr $^{-/}$mice compared to WT mice $[7.762$ (1.461-25.468) vs. 2.369 (0.358-6.658); $P=0.020$ and 0.845 (0.154-3.462) vs. 0.310 (0.088-11.225); $P=0.024)]$ (Table 6.4), but were no longer significant after correcting for multiple testing.

Table 6.4 Transcription of genes involved in oxidative stress

\begin{tabular}{lccc}
\hline & WT $(\mathrm{n}=12)$ & $\operatorname{Ldlr}^{-1-}(\mathrm{n}=12)$ & $P$ value \\
\hline Sigmoid & & & \\
GCLC & $4.286(1.772-12.990)$ & $6.428(3.907-22.187)$ & 0.133 \\
GCLM & $2.369(0.358-6.658)$ & $7.762(1.461-25.468)$ & 0.021 \\
GS & $0.548(0.178-2.406)$ & $0.551(0.255-1.656)$ & 0.525 \\
GPX1 & $0.462(0.271-1.221)$ & $0.676(0.287-1.861)$ & 0.106 \\
GPX2 & $0.611(0.384-1.242)$ & $0.510(0.215-1.103)$ & 0.184 \\
GPX3 & $0.430(0.133-4.067)$ & $0.471(0.192-1.639)$ & 0.603 \\
GR & $0.508(0.270-1.463)$ & $0.645(0.275-1.233)$ & 0.273 \\
XDH & $0.310(0.088-11.225)$ & $0.845(0.154-3.462)$ & 0.024 \\
\hline
\end{tabular}

All data are normalized expression ratios, presented as medians (range).

\section{Intestinal contractility}

Segments of duodenum from 12 WT and $12 \mathrm{Ldll}^{-/-}$mice were collected for analyzing circular smooth muscle contractility in organ bath experiments. The spontaneous contractility was not significantly different in WT (median IMA $0.06 \mathrm{mN} \mathrm{s}^{-1} \mathrm{~mm}^{-2}$, range 
0.02-0.25) and $L d l r^{-/}$mice (median IMA $0.10 \mathrm{mN} \mathrm{s}^{-1} \mathrm{~mm}^{-2}$, range 0.03-0.24). Also, the contraction evoked by $62.5 \mathrm{mM} \mathrm{K}^{+}$was not significantly different in WT (median force $0.36 \mathrm{mN} \mathrm{mm}{ }^{-2}$, range $0.14-0.55$ ) and $L d l r^{-/}$mice (median force $0.37 \mathrm{mN} \mathrm{mm}^{-2}$, range $0.12-0.76)$. As shown in Figure 6.1, the addition of carbachol $(10 \mathrm{nM}-10 \mu \mathrm{M})$ to the organ bath induced a concentration-dependent contraction in the duodenum, which was comparable between both mouse models. Finally, the relaxation evoked by isoprenaline in carbachol-contracted duodenal rings was also not different between WT and $L d l r^{-/-}$mice (Figure 6.2).

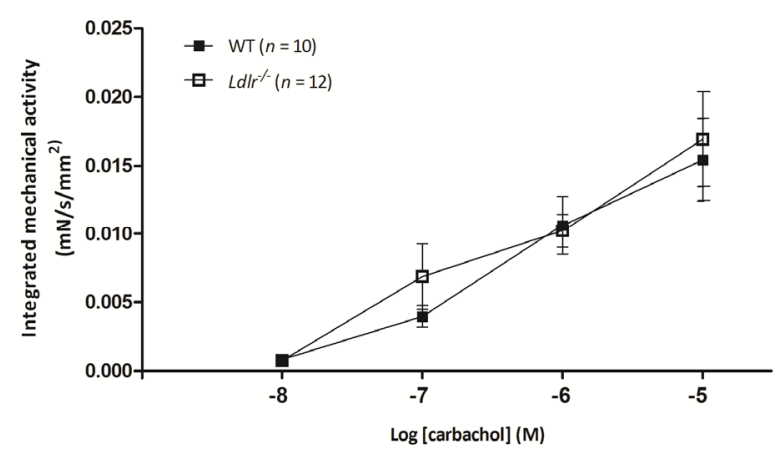

Figure 6.1 Carbachol-induced contractile responses $(10 \mathrm{nM}-10 \mu \mathrm{M})$ in the circular smooth muscle of duodenal segments of WT and $L d l r^{-1-}$ mice. Values are expressed as integrated mechanical activity (IMA) per second $\left(\mathrm{mN} \mathrm{s}^{-1}\right)$, normalized per CSA $\left(\mathrm{mN} \mathrm{s}^{-1} \mathrm{~mm}^{-2}\right)$. Data are presented as mean \pm SEM $(P>0.05)$.

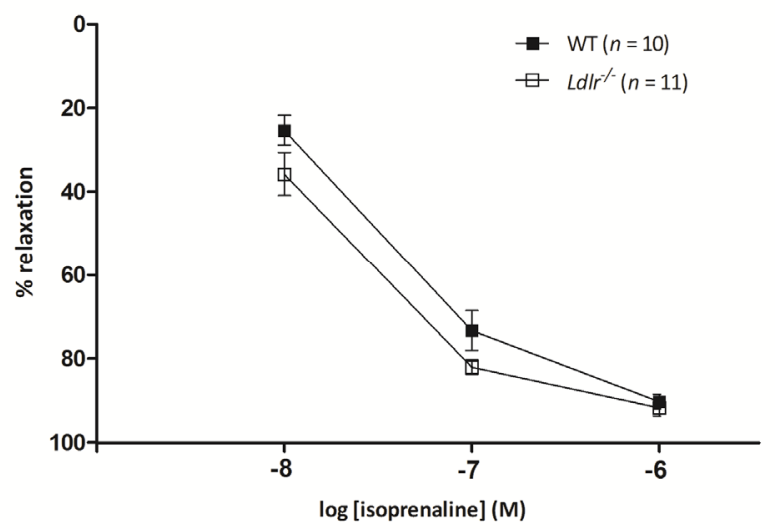

Figure 6.2 Relaxations induced by isoprenaline in carbachol-contracted circular smooth muscle of duodenal segments of WT and Ldlr $^{-/}$mice. Values are expressed as a percentage of the relaxation induced by $0.1 \mathrm{mM}$ papaverine at the end of the experiment, which was arbitrarily defined as $100 \%$ relaxation. Data are presented as mean \pm SEM $(P>0.05)$. 


\section{Discussion}

In the present study we did not find an altered intestinal epithelial barrier function in ileum and sigmoid between $W T$ and $L d l r^{-/}$mice after a high-fat and high-cholesterol diet for 3 weeks. Furthermore, no differences were found in the transcription of oxidative stress-related genes apart from an upregulation of GCLM and XDH in sigmoid of $\mathrm{Ldll}^{-1-}$ mice. The circular smooth muscle contractility of duodenal segments was also not different between both mouse models.

This study was initiated to explore whether intestinal alterations could play a contributing role in the transition of hepatic steatosis to steatohepatitis. The intestinal epithelial barrier function, oxidative stress and motility, factors which may facilitate bacterial translocation and thereby hepatic inflammation, were studied in small and large intestinal segments. We used two mouse models that have been shown to develop either hepatic steatosis only (i.e. female $C 57 \mathrm{Bl} / 6 \mathrm{~J}$ mice) or both early hepatic inflammation and steatosis (i.e. female $\mathrm{Ldll}^{-/-}$mice) when fed a high-fat and highcholesterol diet. ${ }^{26}$ In the present study, we confirmed that a high-fat and highcholesterol diet for 3 weeks led to comparable hepatic steatosis in WT and $\mathrm{Ldll}^{-1-}$ mice, and an increased hepatic inflammation in $\mathrm{Ldlr}^{-/-}$mice [manuscript in preparation].

Ex vivo measurements of paracellular permeability of ileum and sigmoid segments mounted in Ussing chambers showed no significant differences between WT and LdIr ${ }^{-/}$ mice. Also gene transcription of TJ and associated proteins in ileum and sigmoid did not differ between the models. However, it has to be acknowledged that we cannot exclude a decreased barrier function in both NAFLD mouse models when compared with control mice on a normal diet. Other animal studies did provide evidence for intestinal barrier dysfunction in mice on a high fructose diet that developed hepatic steatosis with some signs of inflammation when compared to a control group on a normal diet. ${ }^{15-18}$ These studies used a specific diet (i.e. fructose solution) to induce hepatic damage, which in itself can affect the epithelial barrier. Our findings suggest that dysfunction of the epithelial barrier does not seem to be an important factor contributing to hepatic inflammation in the applied mouse models.

NASH has been associated with systemic and hepatic inflammation and oxidative stress, ${ }^{34-36}$ which may reach the intestine via blood and bile and may affect epithelial barrier function. Therefore, the transcription of genes involved in the metabolism of GSH and uric acid, which are both important antioxidants and protect cells against oxidative damage, were analyzed in the intestinal segments. In sigmoid only, we found the gene transcription of GCLM and XDH to be upregulated in $\mathrm{LdIr}^{-/-}$compared to WT mice. GCLM is a subunit of glutamate cysteine ligase $(G C L)$, which is the rate-limiting enzyme for $\mathrm{GSH}$ synthesis and $\mathrm{XDH}$ is the rate-limiting enzyme in the purine metabolism with uric acid being the final oxidation product. However, we observed a large variation in the expression ratios and after correcting for multiple testing, results 
were no longer statistically significant. Moreover, these findings were not confirmed in ileum, nor were differences found in other oxidative stress-related genes investigated. A previous study in rats did provide evidence of oxidative stress in the small intestine of rats that developed hepatic steatosis and steatohepatitis after 8 and 12 weeks of high-fat feeding, respectively. ${ }^{19}$ In addition, oxidative stress parameters (i.e. SOD and MDA levels) were more pronounced in rats with steatohepatitis compared to those with hepatic steatosis. The discrepancy in results may be explained by the shorter feeding period in our study as we aimed to focus on early onset hepatic inflammation, which might have been insufficient to evoke a clear effect on oxidative stress and an altered transcriptional response of the genes studied. Due to the limited amount of tissue available, we were not able to measure functional analyses of oxidative stress (e.g. the GSH/GSSG ratio) in the intestinal segments.

As intestinal dysmotility may enhance bacterial overgrowth of the small intestine and an altered intestinal microbiota composition in NAFLD, we evaluated the circular smooth muscle contractility of duodenal segments mounted in organ baths. No differences were found regarding the spontaneous contractility, $\mathrm{K}^{+}$- and carbacholinduced contractions and isoprenaline-induced relaxation between WT and $\mathrm{Ldll}^{-/}$mice. Previous studies on intestinal dysmotility in NAFLD are limited, focused on measuring the orocaecal transit time and reported inconsistent results. ${ }^{12,13,23-25}$ Our findings indicate that small intestinal dysmotility by promoting bacterial overgrowth and subsequently enhancing the risk of bacterial translocation may not be a major factor contributing to hepatic inflammation in the applied mouse models. However, this conclusion needs to be drawn cautiously because we observed a broad dispersion of the values measured in the contractility experiments and we only analyzed circular smooth muscle duodenal contractility. Therefore, potential alterations in the contractility of longitudinal muscles or in the motility of large intestinal segments cannot be excluded. As recently also alterations in the intestinal microbiota composition of the large intestine have been reported in NAFLD, ${ }^{21}$ it would be interesting to investigate large intestine motility.

The strength of our study is that we compared a mouse model displaying only hepatic steatosis with a mouse model displaying both hepatic steatosis and inflammation after a 3 week high-fat and high-cholesterol diet, thereby representing early steatohepatitis, and investigated the small as well as the large intestine combining analyses at functional and gene level.

Some limitations of this study should also be taken into account. As the primary aim of this study was to investigate whether intestinal alterations could play a contributing role in the transition of hepatic steatosis to steatohepatitis, we did not include a control group of mice fed a normal diet. Therefore, the lack of differences in intestinal epithelial barrier function, oxidative stress and motility between WT and $\mathrm{Ldll}^{-/}$mice does not exclude the presence of intestinal alterations in both NALFD mouse models 
when compared with control mice on a normal diet. Furthermore, we did not follow the mice in the progression from hepatic steatosis towards NASH over time.

In the present study, mice were fed a high-fat and high-cholesterol diet for 3 weeks resulting in both early hepatic inflammation and steatosis in the $L d l r^{-/}$mice, which allowed us to address the role of the intestine in the early development of steatohepatitis. When mice were fed a high-fat and high-cholesterol diet for a longer time period, which has been shown to result in sustained hepatic inflammation and fibrosis (i.e. representing a more advanced stage of NALFD), ${ }^{37}$ alterations in the intestine may have been more pronounced but may also result from hepatic inflammation. However, we cannot exclude that the 3 -week period of high-fat and high-cholesterol feeding may be too short to evoke large alterations in the transcription of genes involved in intestinal epithelial barrier function and/or oxidative stress.

The present study was part of a larger intervention study ${ }^{27}$ of which only the placebotreated mice $(n=24)$ were available for studying the intestine. Due to the limited capacity/availability of Ussing chambers and organ baths, we were not able to do all the analyses on every location and to perform functional analyses of oxidative stress. In the future, it would also be interesting to perform microbial analyses as the intestinal microbiota is increasingly recognized to be an important factor in the development and progression of NAFLD.

In conclusion, no major differences regarding epithelial barrier function, oxidative stress and motility were found in both the small and large intestine between WT and $\mathrm{LdIr}^{-/}$mice after a 3-week period of high-fat and high-cholesterol feeding. These findings suggest that the intestine does not play an important role in the transition of hepatic steatosis to steatohepatitis in these mouse models. 


\section{References}

1. Younossi ZM, Stepanova M, Afendy M, et al. Changes in the prevalence of the most common causes of chronic liver diseases in the United States from 1988 to 2008. Clin Gastroenterol Hepatol 2011;9:52430 e1; quiz e60.

2. Vernon G, Baranova A, Younossi ZM. Systematic review: the epidemiology and natural history of nonalcoholic fatty liver disease and non-alcoholic steatohepatitis in adults. Aliment Pharmacol Ther 2011;34:274-85.

3. Chalasani N, Younossi Z, Lavine JE, et al. The diagnosis and management of non-alcoholic fatty liver disease: practice Guideline by the American Association for the Study of Liver Diseases, American College of Gastroenterology, and the American Gastroenterological Association. Hepatology 2012;55:2005-23.

4. Mencarelli A, Cipriani S, Renga B, et al. VSL\#3 resets insulin signaling and protects against NASH and atherosclerosis in a model of genetic dyslipidemia and intestinal inflammation. PLoS One 2012; 7:e45425.

5. Gabele E, Dostert K, Hofmann C, et al. DSS induced colitis increases portal LPS levels and enhances hepatic inflammation and fibrogenesis in experimental NASH. J Hepatol 2011;55:1391-9.

6. Purohit V, Bode JC, Bode C, et al. Alcohol, intestinal bacterial growth, intestinal permeability to endotoxin, and medical consequences: summary of a symposium. Alcohol 2008;42:349-61.

7. Suzuki T, Hara H. Dietary fat and bile juice, but not obesity, are responsible for the increase in small intestinal permeability induced through the suppression of tight junction protein expression in LETO and OLETF rats. Nutr Metab (Lond) 2010;7:19.

8. Qin H, Zhang Z, Hang X, Jiang Y. L. plantarum prevents enteroinvasive Escherichia coli-induced tight junction proteins changes in intestinal epithelial cells. BMC Microbiol 2009;9:63.

9. Bruewer $M$, Luegering $A$, Kucharzik $T$, et al. Proinflammatory cytokines disrupt epithelial barrier function by apoptosis-independent mechanisms. J Immunol 2003;171:6164-72.

10. Rao RK, Basuroy S, Rao VU, Karnaky Jr KJ, Gupta A. Tyrosine phosphorylation and dissociation of occludin-ZO-1 and E-cadherin-beta-catenin complexes from the cytoskeleton by oxidative stress. Biochem J 2002;368:471-81.

11. Miele L, Valenza V, La Torre G, et al. Increased intestinal permeability and tight junction alterations in nonalcoholic fatty liver disease. Hepatology 2009;49:1877-87.

12. Volynets $\mathrm{V}$, Kuper MA, Strahl S, et al. Nutrition, intestinal permeability, and blood ethanol levels are altered in patients with nonalcoholic fatty liver disease (NAFLD). Dig Dis Sci 2012;57:1932-41.

13. Wigg AJ, Roberts-Thomson IC, Dymock RB, McCarthy PJ, Grose RH, Cummins AG. The role of small intestinal bacterial overgrowth, intestinal permeability, endotoxaemia, and tumour necrosis factor alpha in the pathogenesis of non-alcoholic steatohepatitis. Gut 2001;48:206-11.

14. Farhadi A, Gundlapalli S, Shaikh M, et al. Susceptibility to gut leakiness: a possible mechanism for endotoxaemia in non-alcoholic steatohepatitis. Liver Int 2008;28:1026-33.

15. Spruss A, Kanuri G, Stahl C, Bischoff SC, Bergheim I. Metformin protects against the development of fructose-induced steatosis in mice: role of the intestinal barrier function. Lab Invest 2012;92:1020-32.

16. Wagnerberger S, Spruss A, Kanuri G, et al. Toll-like receptors 1-9 are elevated in livers with fructoseinduced hepatic steatosis. Br J Nutr 2012;107:1727-38.

17. Volynets V, Spruss A, Kanuri G, Wagnerberger S, Bischoff SC, Bergheim I. Protective effect of bile acids on the onset of fructose-induced hepatic steatosis in mice. J Lipid Res 2010;51:3414-24.

18. Haub S, Kanuri G, Volynets V, Brune T, Bischoff SC, Bergheim I. Serotonin reuptake transporter (SERT) plays a critical role in the onset of fructose-induced hepatic steatosis in mice. Am J Physiol Gastrointest Liver Physiol 2010;298:G335-44.

19. Li S, Wu WC, He CY, Han Z, Jin DY, Wang L. Change of intestinal mucosa barrier function in the progress of non-alcoholic steatohepatitis in rats. World J Gastroenterol 2008;14:3254-8.

20. Sheth P, Basuroy S, Li C, Naren AP, Rao RK. Role of phosphatidylinositol 3-kinase in oxidative stressinduced disruption of tight junctions. J Biol Chem 2003;278:49239-45.

21. Mouzaki M, Comelli EM, Arendt BM, et al. Intestinal microbiota in patients with nonalcoholic fatty liver disease. Hepatology 2013;58:120-7. 
22. Shanab AA, Scully $P$, Crosbie $O$, et al. Small intestinal bacterial overgrowth in nonalcoholic steatohepatitis: association with toll-like receptor 4 expression and plasma levels of interleukin 8. Dig Dis Sci 2011;56:1524-34.

23. Wu WC, Zhao W, Li S. Small intestinal bacteria overgrowth decreases small intestinal motility in the NASH rats. World J Gastroenterol 2008;14:313-7.

24. Fu XS, Jiang F. Cisapride decreasing orocecal transit time in patients with nonalcoholic steatohepatitis. Hepatobiliary Pancreat Dis Int 2006;5:534-7.

25. Soza A, Riquelme A, Gonzalez R, et al. Increased orocecal transit time in patients with nonalcoholic fatty liver disease. Dig Dis Sci 2005;50:1136-40.

26. Wouters K, van Gorp PJ, Bieghs V, et al. Dietary cholesterol, rather than liver steatosis, leads to hepatic inflammation in hyperlipidemic mouse models of nonalcoholic steatohepatitis. Hepatology 2008;48:474-86.

27. Lemmens $\mathrm{KJ}$, van de Wier $\mathrm{B}$, Koek $\mathrm{GH}$, et al. The flavonoid monoHER promotes the adaption to oxidative stress during the onset of NAFLD. Biochem Biophys Res Commun 2015;456:179-82.

28. Kleiner DE, Brunt EM, Van Natta M, et al. Design and validation of a histological scoring system for nonalcoholic fatty liver disease. Hepatology 2005;41:1313-21.

29. Bieghs V, Wouters K, van Gorp PJ, et al. Role of scavenger receptor A and CD36 in diet-induced nonalcoholic steatohepatitis in hyperlipidemic mice. Gastroenterology 2010;138:2477-86, 86 e1-3.

30. Wallon C, Braaf Y, Wolving M, Olaison G, Soderholm JD. Endoscopic biopsies in Ussing chambers evaluated for studies of macromolecular permeability in the human colon. Scand J Gastroenterol 2005;40:586-95.

31. Wallon $\mathrm{C}$, Yang PC, Keita AV, et al. Corticotropin-releasing hormone (CRH) regulates macromolecular permeability via mast cells in normal human colonic biopsies in vitro. Gut 2008;57:50-8.

32. Gonzalo S, Grasa L, Arruebo MP, Plaza MA, Murillo MD. Lipopolysaccharide-induced intestinal motility disturbances are mediated by c-Jun NH2-terminal kinases. Dig Liver Dis 2011;43:277-85.

33. Moreels TG, De Man JG, De Winter BY, Herman AG, Pelckmans PA. How to express pharmacological contractions of the inflamed rat intestine. Naunyn Schmiedebergs Arch Pharmacol 2001;364:524-33.

34. Rolo AP, Teodoro JS, Palmeira CM. Role of oxidative stress in the pathogenesis of nonalcoholic steatohepatitis. Free Radic Biol Med 2012;52:59-69.

35. Sumida $\mathrm{Y}$, Niki E, Naito $\mathrm{Y}$, Yoshikawa $\mathrm{T}$. Involvement of free radicals and oxidative stress in NAFLD/NASH. Free Radic Res 2013;47:869-80.

36. Fujii H, Kawada N. Inflammation and fibrogenesis in steatohepatitis. J Gastroenterol 2012;47:215-25.

37. Bieghs V, Van Gorp PJ, Wouters K, et al. LDL receptor knock-out mice are a physiological model particularly vulnerable to study the onset of inflammation in non-alcoholic fatty liver disease. PLoS One 2012;7:e30668. 


\section{Supplemental data}

\begin{tabular}{lcc}
\hline & $W T(\mathrm{n}=12)$ & Ldlr $^{-/}(\mathrm{n}=12)$ \\
\hline Initial body weight $(\mathrm{g})$ & $20.8 \pm 1.3$ & $20.9 \pm 1.1$ \\
Final body weight $(\mathrm{g})$ & $21.3 \pm 1.5$ & $21.3 \pm 1.5$ \\
Body weight change $(\mathrm{g})$ & $0.4 \pm 0.7$ & $0.4 \pm 1.2$ \\
Liver weight $(\mathrm{g})$ & $1.00 \pm 0.14$ & $1.05 \pm 0.12$ \\
Liver/total body weight-ratio (\%) & $4.69 \pm 0.40$ & $4.95 \pm 0.35$ \\
Average food intake (g/day/cage) & $4.0(0.3)$ & $4.3(0.3)$ \\
\hline
\end{tabular}


Chapter 7

Critical appraisal of ${ }^{13} \mathrm{C}$ breath tests for microsomal liver function: aminopyrine revisited

K.E. Pijls

H. de Vries

S. Nikkessen

A. Bast

W.K.W.H. Wodzig

G.H. Koek

Liver Int 2014;34:487-94 


\section{Abstract}

As liver diseases are a major health problem and especially the incidence of metabolic liver diseases like non-alcoholic fatty liver disease (NAFLD) is rising, the demand for non-invasive tests is growing to replace liver biopsy. Non-invasive tests such as carbon-labeled breath tests can provide a valuable contribution to the evaluation of metabolic liver function. This review aims to critically appraise the value of the ${ }^{13} \mathrm{C}$-labeled microsomal breath tests for the evaluation of metabolic liver function, and to discuss the role of cytochrome P450 enzymes in the metabolism of the different probe drugs, especially of aminopyrine.

Although a number of different probe drugs have been used in breath tests, the perfect drug to assess the functional metabolic capacity of the liver has not been found. Data suggest that both the ${ }^{13} \mathrm{C}_{2}$-aminopyrine and the ${ }^{13} \mathrm{C}$-methacetin breath test can play a role in assessing the capacity of the microsomal liver function and may be useful in the follow-up of patients with chronic liver diseases. Furthermore, CYP2C19 seems to be an important enzyme in the $N$-demethylation of aminopyrine and polymorphisms in this gene may influence breath test values, which should be kept in mind when performing the ${ }^{13} \mathrm{C}_{2}$-aminopyrine breath test in clinical practice. 


\section{Introduction}

The liver plays a key role in the metabolism of both endogenous and exogenous substances, which can be disturbed in liver diseases. A number of tests are used to assess liver function. Measurement of biochemical enzyme levels such as serum transaminases give information on the presence or absence of hepatic tissue injury. Histological examination of biopsy tissue reflects the morphologic structure of the liver and is, at present, the gold standard for diagnosis. Although these tests are commonly used in clinical practice, they do not provide information regarding the functional metabolic capacity of the liver. Therefore, several tests have been introduced that reflect the capacity of a specific metabolic liver function. As these tests are based on the administration of a probe drug and the subsequent measurement of its substrate, these specific drugs should meet several criteria. ${ }^{1}$ For example, the probe drug should be safe and have almost no interaction with other diseases, environmental and genetic factors. The absorption should be rapid and consistent and the probe drug should be water soluble and have simple pharmacokinetics. Furthermore, the metabolic pathway of the probe drug should be clear, fast, occur completely in the liver and should be independent of liver blood flow. Finally, the preparation, performance and analysis should be easy and inexpensive. ${ }^{1}$

At first, radioactive carbon-labeled probe drugs were used in breath tests. ${ }^{2,3}$ But due to the presence of radioactivity, the isotope ${ }^{14} \mathrm{C}$ was replaced by the stable isotope ${ }^{13} \mathrm{C}$ and thereby it became a safe test, which can be performed repeatedly, even in children and pregnant women. ${ }^{4}$

Aminopyrine was the first probe drug used in breath tests. ${ }^{2,3}$ After oral administration of carbon-labeled aminopyrine, metabolism via a two-step $\mathrm{N}$-demethylation catalyzed by cytochrome P450 enzymes occurs (Figure 7.1). The formaldehyde $\left(\mathrm{H}_{2}{ }^{13} \mathrm{CO}\right)$, generated by the $\mathrm{N}$-demethylation is oxidized to formic acid $\left(\mathrm{H}^{13} \mathrm{COOH}\right)$ and bicarbonate $\left(\mathrm{H}^{13} \mathrm{CO}_{3}{ }^{-}\right)$, which then may either be exhaled as ${ }^{13} \mathrm{CO}_{2}$ in breath or equilibrated with the body's formate and bicarbonate pools. Since $\mathrm{N}$-demethylation is the rate-limiting step that occurs almost exclusively in the liver, ${ }^{5}{ }^{13} \mathrm{CO}_{2}$ output in breath reflects the activity of cytochrome P450 enzymes and thereby residual liver microsomal function. ${ }^{6}$

Besides the ${ }^{13} \mathrm{C}$-aminopyrine breath test, several other ${ }^{13} \mathrm{C}$-labeled breath tests have been introduced to assess the microsomal function of the liver, such as the ${ }^{13} \mathrm{C}$ methacetin breath test and the ${ }^{13} \mathrm{C}$-caffeine breath test. These breath tests have been used to predict liver fibrosis and cirrhosis, ${ }^{7}$ and to detect the residual liver function in pre- and post-transplantation settings. ${ }^{8}$

This review critically appraises the value of the different ${ }^{13} \mathrm{C}$-labeled microsomal breath tests as non-invasive tests for the evaluation of metabolic liver function. In addition, the role of cytochrome P450 enzymes involved in the metabolism of the different probe drugs, especially of aminopyrine, will be discussed. 
<smiles>Cc1c(N([13CH3])[14CH3])c(=O)n(-c2ccccc2)n1C</smiles>

(aminopyrine)

Figure 7.1 N-demethylation of ${ }^{13} \mathrm{C}_{2}$-aminopyrine.

\section{Cytochrome P450 enzymes}

The majority of drugs is metabolized in the liver by two processes classified as phase I and phase II reactions. In phase I reactions (such as oxidation, reduction and hydrolysis), functional groups are added to the drugs in preparation for phase II reactions. In phase II reactions, conjugation with moieties as sulphate, glucuronic acid and glutathione takes place, which increases the water solubility of the resulting drug metabolites and hence facilitates their excretion. ${ }^{9}$

Oxidation reactions for drugs are mainly catalyzed by enzymes of the hepatic microsomal cytochrome P450 system. ${ }^{9}$ The cytochrome P450 enzymes are categorized into gene families and subfamilies on the basis of their amino acid sequence by an internationally accepted nomenclature system. ${ }^{10,11}$ An example of the nomenclature is presented in Figure 7.2.

The activity of cytochrome P450 enzymes varies between individuals, leading to interindividual variation in the metabolic conversion and excretion rate of drugs. ${ }^{12} \mathrm{~A}$ part of this variation is caused by the presence of polymorphisms in the cytochrome P450 genes, which can result in either defective, qualitatively altered, diminished or enhanced enzyme activities, ${ }^{13}$ leading to generally four phenotypes: poor metabolizers (PMs), intermediate metabolizers (IMs), extensive metabolizers (EMs) and ultrarapid metabolizers (UMs). ${ }^{12}$ 


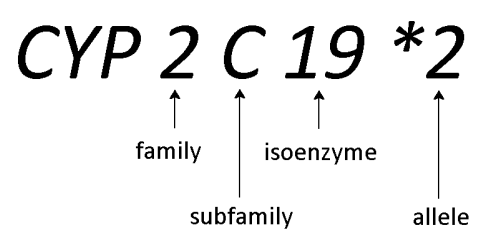

Figure 7.2 An example of the nomenclature of the cytochrome P450 system.

\section{Principle of ${ }^{13} \mathrm{C}$-labeled breath tests for metabolic liver function}

Breath tests are functional tests that are based on the administration of a probe drug, which is mainly metabolized by the liver, and on the subsequent measurement of the substrate or its metabolite. ${ }^{1}$ The principle for most functional tests is the hepatic clearance $(C l)$, which is related to the hepatic perfusion $(Q)$ and the extraction ratio $(E)$ of the probe drug (i.e. $C l=Q \times E$ ). ${ }^{1}$ In this review, we focus on investigating the metabolic liver function and therefore, a probe drug needs to fulfill several criteria, in particular has to be independent of hepatic perfusion, i.e. has a low extraction ratio. If patients have an altered blood flow in case of a chronic liver disease due to portal hypertension and porto-systemic shunts, a probe drug with a high extraction ratio can be used to obtain information on hepatic perfusion.

In breath tests, the administered probe drug carries a functional group in which the ${ }^{12} \mathrm{C}$ atom is replaced by the stable ${ }^{13} \mathrm{C}$ isotope. The functional group is cleaved enzymatically and undergoes a metabolic process, yielding ${ }^{13} \mathrm{CO}_{2} \cdot{ }^{13} \mathrm{CO}_{2}$ is then mixed with the body's bicarbonate pool and exhaled in breath. The appearance of ${ }^{13} \mathrm{CO}_{2}$ in breath reflects the rate-limiting step of the process that is investigated, provided that all other processes are not disturbed. ${ }^{1,4}$ As the rate-limiting step of the process can occur in specific locations of the liver (e.g. microsomes, cytosol, mitochondrion) information may be obtained on physiological and pathophysiological metabolic processes occurring predominantly at those sites. ${ }^{1,14}{ }^{13} \mathrm{C}$-labeled breath tests that have been used to investigate the microsomal process, reflect the activity of one or more enzymes of the cytochrome P450 system, and thereby indirectly measure (microsomal) liver function. 


\section{${ }^{13} \mathrm{C}$-labeled microsomal breath tests}

Breath tests with ${ }^{13} \mathrm{C}$-labeled probe drugs that have been investigated for the assessment of microsomal liver function, such as ${ }^{13} \mathrm{C}$-labeled phenacetin, methacetin, caffeine and aminopyrine will be discussed in the following paragraphs.

\section{${ }^{13} \mathrm{C}$-phenacetin breath test}

After oral administration, ${ }^{13} \mathrm{C}$-labeled phenacetin, $\quad \mathrm{N}$-[4-ethoxy- $\left(1-{ }^{13} \mathrm{C}\right)$-phenyl] acetamide, undergoes $\mathrm{O}$-de-ethylation, mainly by the cytochrome $\mathrm{P} 450$ isoenzyme CYP1A2. ${ }^{1}$ After de-ethylation, the label is released as acetaldehyde or ethanol. Ethanol is then oxidized into acetaldehyde and subsequently metabolized to acetate, which is finally oxidized via the tricarboxylic acid cycle to ${ }^{13} \mathrm{CO}_{2} \cdot{ }^{15}$ Phenacetin is reported to be a high extraction drug, with an extraction ratio of $>0.8 .{ }^{15,16}$

\section{Studies of the ${ }^{13} \mathrm{C}$-phenacetin breath test}

Few studies have been published on the application of ${ }^{13} \mathrm{C}$-phenacetin in breath tests for assessing metabolic liver function. A study by Kajiwara et al., ${ }^{17}$ showed a decreased ${ }^{13} \mathrm{CO}_{2}$ excretion in breath in patients with cirrhosis compared to healthy subjects after oral administration of $\left(1-{ }^{13} \mathrm{C}\right.$-ethoxy) phenacetin. In the urine test, patients had increased amounts of its metabolite $\left[\left(1-{ }^{13} \mathrm{C}\right)\right.$ ethoxy $]$ phenetidine, suggesting that because of the impaired liver function, the alternative pathway (i.e. metabolizing phenacetin into phenetidine) is enhanced. ${ }^{17}$ Overall, information is lacking about the usability of the ${ }^{13} \mathrm{C}$-phenacetin breath test for the assessment of metabolic liver function. For example, the effects of altered blood flow on its metabolism have never been investigated and the metabolic pathway is still unclear.

\section{${ }^{13} \mathrm{C}$-methacetin breath test}

Methacetin, $\mathrm{N}$-[4-methoxy- $\left({ }^{13} \mathrm{C}\right)$-phenyl] acetamide, is a derivate of the analgesic and antipyretic drug phenacetin. After oral administration, ${ }^{13} \mathrm{C}$-labeled methacetin is metabolized by a rapid $O$-demethylation to acetaminophen. ${ }^{1,18} \mathrm{O}$-demethylation of methacetin is reported to be catalyzed by the cytochrome P450 isoenzyme CYP1A2. ${ }^{1,7}$ Methacetin is a high extraction drug $(E>0.8) .{ }^{1,16}$ The dosage of methacetin used in breath tests is small, ranging from $1 \mathrm{mg} / \mathrm{kg}^{18}$ to a standard fixed dose of $75 \mathrm{mg}^{7}$ dissolved in water and is below therapeutic level. Until now no adverse reactions have been observed. ${ }^{18}$

\section{Studies of the ${ }^{13} \mathrm{C}$-methacetin breath test}

The ${ }^{13} \mathrm{C}$-methacetin breath test has been applied in different stages and types of liver diseases. Breath test values were found to be decreased in patients with chronic HCV infection, ${ }^{19}$ primary biliary cirrhosis ${ }^{20}$ and cirrhosis ${ }^{19,21}$ compared to healthy controls, 
suggesting a diminished liver function. Lara Baruque et al. ${ }^{22}$ further showed that the breath test was able to discriminate between Child B and C cirrhotic patients. In other studies, the ${ }^{13} \mathrm{C}$-methacetin breath test was able to predict the presence of advanced fibrosis $^{7}$ and/or cirrhosis ${ }^{7,19,21,23}$ in patients with chronic liver diseases using liver biopsy as gold standard.

In the transplant setting, the ${ }^{13} \mathrm{C}$-methacetin breath test was studied for the assessment of graft function. Petrolati et al., ${ }^{8}$ showed that pre-transplantation values in cirrhotic patients were significantly lower than post-transplantation values.

In patients with cirrhosis and hepatocellular carcinoma (HCC), the breath test was used before and after transarterial chemoembolization (TACE) and/or radiofrequency ablation (RFA). ${ }^{24}$ At baseline, values were significantly lower in cirrhotic patients with and without HCC compared to controls. After TACE, but not after RFA, values were significantly decreased suggesting that because of TACE, methacetin demethylation decreased more severely reflecting a diminished functional hepatic residual mass. ${ }^{24}$ However, the alterations in blood flow and decreased functional capacity of the tumor might have contributed to these findings. The study of Ciccocioppo et al. ${ }^{18}$ showed significantly decreased breath test values in healthy elderly subjects compared to adult subjects suggesting an impaired hepatic function in the elderly owing to an increased vascular resistance in the hepatic artery flow. ${ }^{18}$ This further emphasizes that the ${ }^{13} \mathrm{C}$ methacetin breath test is sensitive to changes in liver blood flow caused by the high extraction ratio of methacetin. ${ }^{1,16}$

In conclusion, the ${ }^{13} \mathrm{C}$-methacetin breath test may be able to assess metabolic liver function in chronic liver diseases and predict the presence of cirrhosis. However, because of the high extraction ratio of methacetin, the breath test is easily influenced by changes in liver blood flow.

\section{${ }^{13} \mathrm{C}$-caffeine breath test}

${ }^{13} \mathrm{C}$-labeled caffeine, $\left(3-\right.$ methyl- $\left.{ }^{13} \mathrm{C}\right)$ caffeine, is metabolized mainly in the liver through $\mathrm{N}$-demethylation by the cytochrome P450 isoenzyme CYP1A2, yielding three major metabolites: theophylline, paraxanthine and theobromine, ${ }^{1,16,25}$ but only the conversion into paraxanthine results in ${ }^{13} \mathrm{CO}_{2}$. Caffeine labeled with ${ }^{13} \mathrm{C}$ in all three $(1,3$ and 7) methyl groups or specifically in the 1-, 3-, or 7-methyl groups can also be used. However, regarding the mono-labeled caffeine breath tests, caffeine labeled in the 3methyl group was suggested as the most suitable for exploring caffeine $\mathrm{N}$ demethylation. ${ }^{1,26}$ Caffeine has a high bioavailability and has a low extraction ratio of < $0.3 .^{16}$ It is administered at a dose of $2 \mathrm{mg} / \mathrm{kg}$ dissolved in water. Side effects have not been observed with the low dosage, which is comparable to two cups of coffee. ${ }^{25}$

\section{Studies of the ${ }^{13} \mathrm{C}$-caffeine breath test}

A well-designed study by Park et al. ${ }^{25}$ showed that the ${ }^{13} \mathrm{C}$-labeled caffeine breath test was significantly correlated with the plasma caffeine clearance, and with several liver 
tests (e.g. serum albumin). In addition, values were decreased significantly in cirrhotic patients when compared with healthy controls and patients with hepatitis B or C. Although values were increased in smokers, the breath test was able to differentiate cirrhotic from healthy and hepatitis smokers. Finally, the breath test was found to be associated inversely with the Child-Pugh score $(r=-0.74, P=0.002) .{ }^{25}$ On the basis of these findings, the authors stated that the ${ }^{13} \mathrm{C}$-caffeine breath test correlated reliably with hepatic dysfunction. Further studies performed by the same group investigated the utility of the ${ }^{13} \mathrm{C}$-caffeine breath test for detecting fibrosis in chronic hepatitis $\mathrm{B}^{27}$ and NAFLD patients. ${ }^{28}$ Chronic hepatitis B patients with fibrosis grade two or three and those with cirrhosis had significantly decreased values compared to patients with fibrosis grade one or less. ${ }^{27}$ Patients with NASH and cirrhosis had also significantly decreased values. In addition, the ${ }^{13} \mathrm{C}$-caffeine breath test correlated significantly with Brunt's fibrosis score and several other liver tests. ${ }^{28}$

The ${ }^{13} \mathrm{C}$-caffeine breath test seems to be an adequate and safe method to measure metabolic liver function especially in chronic liver diseases and may predict significant fibrosis in patients with chronic hepatitis B and NAFLD. As only a few studies have been performed, more studies are needed to establish the usefulness of the ${ }^{13} \mathrm{C}$-caffeine breath test in the assessment of metabolic liver function.

\section{${ }^{13} \mathrm{C}_{2}$-aminopyrine breath test}

Aminopyrine, dimethyl-aminoantipyrine (DMAA), is an analgesic and antipyretic drug. After oral administration, [4-dimethyl- $\left({ }^{13} \mathrm{C}_{2}\right)$-amino] antipyrine is rapidly and almost completely absorbed from the gastrointestinal tract and is then evenly distributed throughout the body water. ${ }^{29}$ Only a minor fraction of the parent drug is excreted in the urine ${ }^{29,30}$ the rest is metabolized in the body. The metabolism of aminopyrine has been extensively studied and is schematically presented in Figure 7.3. The main metabolites that have been detected in the urine of humans after oral administration are methyl-aminoantipyrine (MAA), aminoantipyrine (AA), acetyl-aminoantipryine (AcAA) and formyl-aminoantipyrine (FAA) (for review, see Volz et al. ${ }^{30}$ ).

The first metabolic reaction is $N$-demethylation of aminopyrine into MAA and formaldehyde. Subsequently, MAA also undergoes $N$-demethylation to form $A A$ and another molecule of formaldehyde. The two-step $N$-demethylation of aminopyrine is suggested to be catalyzed by several cytochrome P450 isoenzymes. ${ }^{31}$ Niwa et al. ${ }^{32}$ showed aminopyrine $\mathrm{N}$-demethylase activities for 10 different isoenzymes of human hepatic cytochrome P450 expressed in yeast Saccharomyces cerevisiae and reported the highest activity for CYP2C19. ${ }^{32}$ However, to determine the contribution to the aminopyrine $\mathrm{N}$-demethylation of the involved isoenzymes, both the content present in the human liver as well as the isoenzymes with high aminopyrine $\mathrm{N}$-demethylase activities, i.e. CYP2C19, CYP2C8, CYP2D6 and CYP2A1, ${ }^{32}$ should be taken into account. As the hepatic clearance of aminopyrine is almost independent of hepatic perfusion, because of the low extraction ratio $(E=0.2)^{1,16}$ it will be determined by the total 
intrinsic clearance. The hepatic clearance of aminopyrine was found to be 3.0 $\mathrm{ml} / \mathrm{min} / \mathrm{kg}$ in healthy subjects, ${ }^{33}$ and will be approximately $240 \mathrm{ml} / \mathrm{min}$ for a person with an average weight of $80 \mathrm{~kg}$. The total intrinsic clearance of aminopyrine is the sum of the intrinsic clearance of each of the involved isoenzymes, i.e. 333, 36, 17 and $13 \mu \mathrm{l} / \mathrm{min} / \mathrm{nmol}^{\mathrm{P}} 450$ for CYP2C19, CYP2C8, CYP2D6 and CYP1A2, respectively. ${ }^{32}$

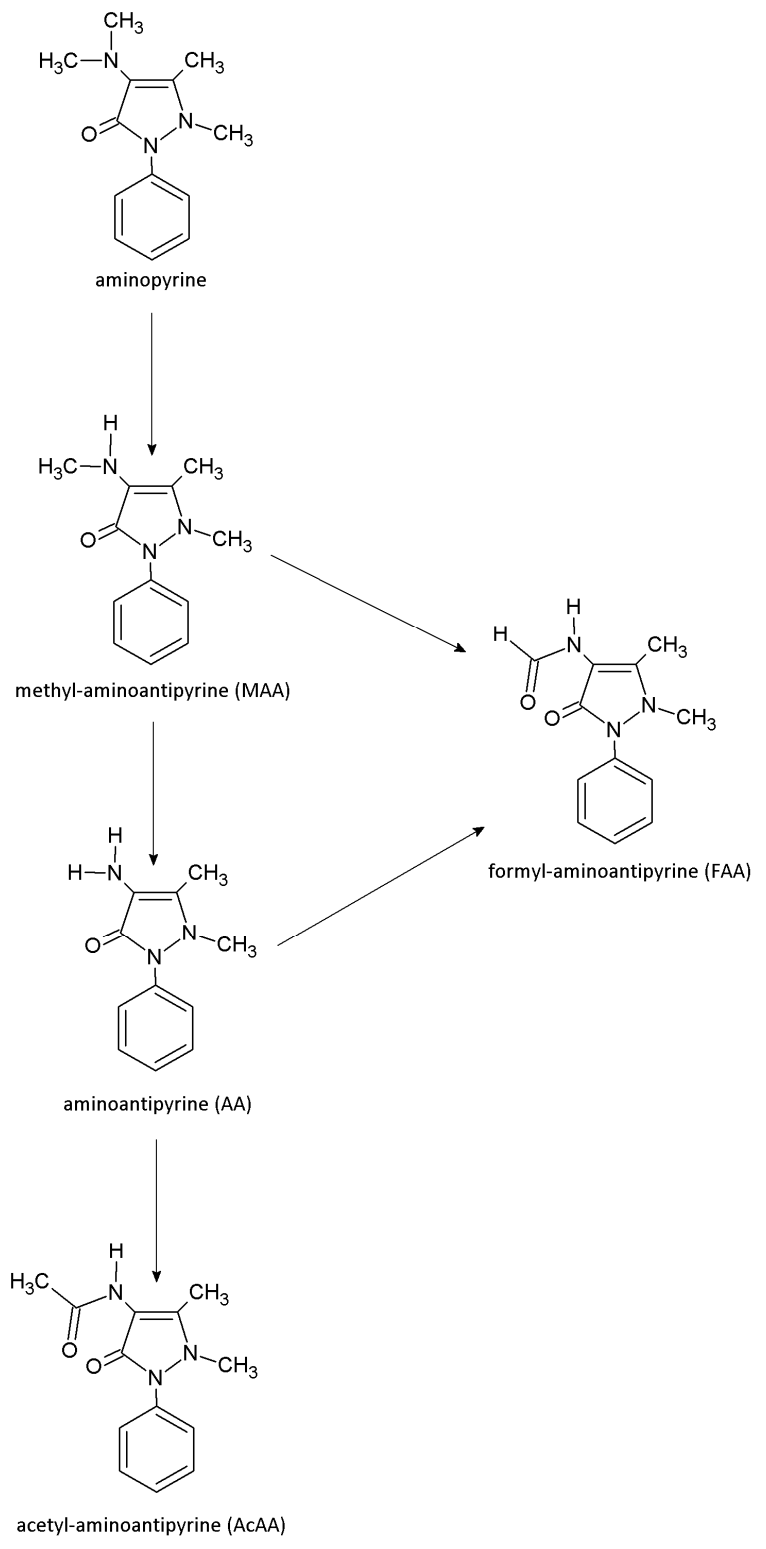

Figure 7.3 Aminopyrine metabolism (based on ${ }^{29-31,36,37}$ ). 
The presence of these isoenzymes in human liver microsomes has been derived from Rodrigues et al. ${ }^{34}$ (Table 7.1). By assuming a microsomal protein content of $19.9 \mathrm{mg} / \mathrm{g}$ of liver ${ }^{35}$ and an average weight of $1400 \mathrm{~g}$ for the adult human liver, we could estimate the contents as well as the intrinsic clearances of the isoenzymes per human liver (Table 7.1). Accordingly, the total intrinsic clearance is calculated to be approximately $261 \mathrm{ml} / \mathrm{min}$, which is comparable with the aminopyrine clearance of $240 \mathrm{ml} / \mathrm{min}$ as estimated earlier. The intrinsic clearance for CYP2C19 is relatively high and accounts for approximately $67 \%$ of the total hepatic intrinsic clearance of aminopyrine (Table 7.1), indicating that especially CYP2C19 contributes to aminopyrine $N$-demethylation. After the two-step $\mathrm{N}$-demethylation, $\mathrm{AA}$ is further metabolized by acetylation into AcAA, which has been reported to be the major metabolite excreted in the urine after oral administration of aminopyrine. ${ }^{30}$ Another quantitatively important urinary metabolite is $\mathrm{FAA}^{36}$ In the literature, two routes have been described for the formation of FAA. According to Volz et al., ${ }^{30}$ FAA is a metabolite of AA. In this case, FAA is formed by formylation (formyl conjugation) of AA. Noda et al., ${ }^{37}$ however, reported that FAA is formed by oxidation of the $N$-methyl group of MAA. Agundez et al. ${ }^{31}$ further showed that $29 \%$ of aminopyrine is converted into FAA via MAA and found a large interindividual difference in the formation of FAA, i.e. the metabolic ratio of FAA/MAA.

Table 7.1 Contents and intrinsic clearances of cytochrome P450 isoenzymes.

\begin{tabular}{lccc}
\hline $\begin{array}{l}\text { Cytochrome P450 } \\
\text { isoenzyme }\end{array}$ & $\begin{array}{c}\mathrm{pmol} \mathrm{CYP/mg} \\
\text { microsomal protein* }\end{array}$ & $\mathrm{nmol} \mathrm{CYP/liver}$ & $\mathrm{Cl}_{\text {int }}(\mathrm{ml} / \mathrm{min})$ \\
\hline CYP2C19 & 19 & 529 & 176 \\
CYP2C8 & 64 & 1783 & 64 \\
CYP2D6 & 10 & 279 & 5 \\
CYP1A2 & 45 & 1254 & 16 \\
Total & & & 261 \\
\hline
\end{tabular}

$\mathrm{Cl}_{\text {int, }}$ intrinsic clearance. ${ }^{*}$ Specific content of each cytochrome P450 isoenzyme in native human liver microsomes from different organ donor subjects $(n=12)\left[\right.$ taken from Rodriques et al., ${ }^{34}$ who obtained data from the Gentest Corp. (October 1997)].

Furthermore, a number of additional less important metabolites have been reported to be excreted in the urine, such as acetylmethyl-aminoantipyrine (AcMAA), hydroxyantipyrine (HA), rubazonic acid (RA), methylrubazonic acid (MRA) and ureidoantipyrine (UA). ${ }^{30}$ The total excretion of aminopyrine and its metabolites that have been reported in the urine of humans ranges from 25 to $50 \%$ of the dose. ${ }^{29,31,38} \mathrm{~A}$ serious side effect of chronic administration of aminopyrine is the occurrence of agranulocytosis, but this has never been reported after a single dose of $75 \mathrm{mg}^{39}$ and/or $2 \mathrm{mg} / \mathrm{kg}^{40}$ of ${ }^{13} \mathrm{C}$-aminopyrine used in the ${ }^{13} \mathrm{C}_{2}$-aminopyrine breath test. ${ }^{41}$ 


\section{Studies of the ${ }^{13} \mathrm{C}_{2}$-aminopyrine breath test}

Several studies have investigated the use of the ${ }^{13} \mathrm{C}_{2}$-aminopyrine breath test. Giannini et al., ${ }^{42}$ for example, assessed the use of the breath test to evaluate the severity of the disease in patients with chronic hepatitis $C$ and found that the test was able to differentiate between normal subjects, chronic hepatitis and cirrhotic patients. Furthermore, both fibrosis score and especially age were found to be independent predictors for the ${ }^{13} \mathrm{C}$-recovery in patients with chronic hepatitis $\mathrm{C}^{39}$ No difference was found between smokers and non-smokers. ${ }^{39}$

In healthy subjects, the use of oral contraceptives has been shown to significantly decrease values of the ${ }^{13} \mathrm{C}_{2}$-aminopyrine as well as the ${ }^{13} \mathrm{C}$-caffeine breath test. ${ }^{43}$ Smoking significantly increased both values in women taking oral contraceptives compared to non-smoking women taking oral contraceptives. ${ }^{43}$ Results regarding the effects of smoking on the ${ }^{13} \mathrm{C}_{2}$-aminopyrine breath test are conflicting and need more investigation.

The ${ }^{13} \mathrm{C}_{2}$-aminopyrine has also been used to predict mortality in 548 cirrhotic patients on a waiting list for liver transplantation. ${ }^{44}$ The breath test had a high prognostic value for survival and short-term survival $[\rho=0.73(95 \% \mathrm{Cl} 0.66-0.81)]$, and correlated with the Child-Pugh score better than with the MELD-score $(\rho=0.72$ and $\rho=0.60$, respectively). Degre et $a l^{45}$ further showed that the ${ }^{13} \mathrm{C}_{2}$-aminopyrine breath test has a greater area under curve than both the Child-Pugh and the MELD scores in cirrhotic patients awaiting transplantation, suggesting that the breath test is as good as or better in predicting mortality. In post-transplantation settings, the combination of the ${ }^{13} \mathrm{C}_{2}$-aminopyrine and the ${ }^{13} \mathrm{C}$-methionine breath test (for mitochondrial function) could be used to evaluate the functioning of the orthotopic transplant liver to predict graft outcome. ${ }^{40}$

Furthermore, the ${ }^{13} \mathrm{C}_{2}$-aminopyrine breath test was assessed in 296 patients with chronic liver disease and showed a negative predictive value of $84 \%$ and a positive predictive value of $90 \%$ for the diagnosis of liver cirrhosis. ${ }^{46}$ Also Giannini et al. ${ }^{47}$ observed that the breath test could discriminate between chronic liver disease patients with or without cirrhosis.

\section{Potential factors influencing ${ }^{13} \mathrm{C}$-labeled breath tests}

Recently, Afolabi et al. ${ }^{14}$ stated that many physiological, anatomical, biochemical, environmental and genetic factors may influence ${ }^{13} \mathrm{C}$-labeled breath test values. These factors may especially be important in patients with liver diseases, in whom portal hypertension, porto-systemic shunts and/or hepato/portopulmonary syndrome can affect liver blood flow. ${ }^{14}$ In general, all factors that influence the endogenous $\mathrm{CO}_{2}$ production, such as preceding food intake, physical activity, fever, thyroid function and lung diseases have also to be taken into account. ${ }^{48}{ }^{13} \mathrm{C}$-labeled microsomal breath tests 
values can further be influenced by both endogenous and exogenous factors that may affect the activity of the cytochrome P450 enzymes, such as genetic polymorphisms and drugs respectively. A recent study in 15 healthy volunteers for example showed the influence of polymorphisms in the CYP2C19 gene on the ${ }^{13} \mathrm{C}_{2}$-aminopyrine breath test, reporting lower values in PMs for CYP2C19 than in IMs and rapid metabolizers (RMs). ${ }^{49}$ Although low ${ }^{13} \mathrm{C}_{2}$-aminopyrine breath test values can thus be the consequence of polymorphisms in the CYP2C19 gene that results into a PM phenotype, this phenotype is only present in $2-6 \%$ of Caucasians ${ }^{50}$ and thereby would not be a major confounding factor in routine clinical practice. However, it should be kept in mind when performing the ${ }^{13} \mathrm{C}_{2}$-aminopyrine breath test in other ethnic groups as for example up to $20 \%$ of Asians are PMs for CYP2C19..$^{51}$ Furthermore, many drugs can induce or inhibit the activity of cytochrome P450 enzymes (see P450 Drug Interaction Table $^{52}$ ) and therefore are important confounders in the interpretation of ${ }^{13} \mathrm{C}$-labeled microsomal breath test values and should be discontinued before the test if possible.

\section{Discussion}

${ }^{13} \mathrm{C}$-labeled microsomal breath tests can provide information on the functional metabolic capacity of the liver. In contrast to liver biopsy, these breath tests reflect a dynamic process, are non-invasive and safe, and can be repeated easily. Advances in automatisation of isotope ratio mass spectrometers have facilitated the use of breath tests as samples can be sent to specially equipped laboratories. Moreover, newer and cheaper analytical techniques seem to emerge rapidly, which further enables the use and analysis in primary care settings. ${ }^{48}$ Therefore, breath tests could play an important role in the follow-up of chronic liver diseases and in pre- and post-transplantation settings.

The search for a probe drug that meets all the criteria, as mentioned in the introduction, is still on-going. Different ${ }^{13} \mathrm{C}$-labeled drugs have been used in breath tests to evaluate the microsomal function of the liver, though not all of them have been investigated thoroughly. Only few data are available on the ${ }^{13} \mathrm{C}$-phenacetin breath test. Phenacetin is a high extraction drug, and therefore its metabolism is dependent on liver blood flow, which could influence breath test values in liver disease patients with portal hypertension and hepatic shunts. The ${ }^{13} \mathrm{C}$-caffeine breath test may be an adequate method to assess metabolic liver function in chronic liver diseases and may predict significant fibrosis in chronic hepatitis C and NAFLD patients, but more studies are needed to assess the usability of the test. The ${ }^{13} \mathrm{C}$-methacetin breath test and the ${ }^{13} \mathrm{C}_{2}$-aminopyrine breath test have been studied most extensively and may especially be useful in predicting liver disease and prognosis. However, in contrast to aminopyrine, methacetin has a high extraction ratio which may influence the ${ }^{13} \mathrm{C}$ methacetin breath test values, especially in chronic liver disease patients with portal hypertension and porto-systemic shunts. ${ }^{41}$ By reviewing the literature on the 
metabolism of aminopyrine, we discerned conflicting data on the metabolic route responsible for the formation of the metabolite FAA. If FAA is formed by oxidation of MAA, less MAA will be available for the second $\mathrm{N}$-demethylation step and reduced amounts of ${ }^{13} \mathrm{CO}_{2}$ in breath will be the consequence. However, the role of this metabolic route in the metabolism of aminopyrine has yet to be established. Furthermore, the $\mathrm{N}$-demethylation of aminopyrine has been suggested to be catalyzed by several cytochrome P450 enzymes of which CYP2C19 seems to be the most important and polymorphisms in this gene may influence breath test values. Besides genetic polymorphisms, especially drugs are important confounding factors in the interpretation of breath tests investigating microsomal liver function and should be taken into account when performing ${ }^{13} \mathrm{C}$-labeled microsomal breath tests in clinical practice.

\section{Conclusion}

${ }^{13} \mathrm{C}$-labeled breath tests can be performed at any place at any time and repeated without affecting patient's health. As liver diseases are a major health problem and especially the incidence of metabolic liver diseases like NAFLD is rising because of the progressive epidemics of obesity and diabetes mellitus, the demand of non-invasive methods is growing to replace liver biopsy.

Breath tests will not be able to replace liver biopsies entirely, as it seems unlikely that there is a perfect probe drug (i.e. fulfilling all criteria). However, they may reduce the need for liver biopsy, especially when used together with other conventional tests. Both the ${ }^{13} \mathrm{C}_{2}$-aminopyrine and the ${ }^{13} \mathrm{C}$-methacetin breath test can play a role in assessing the capacity of the microsomal liver function and may be useful in the follow-up of patients with chronic liver diseases. 


\section{References}

1. Armuzzi A, Candelli M, Zocco MA, et al. Review article: breath testing for human liver function assessment. Aliment Pharmacol Ther 2002;16:1977-96.

2. Hepner GW, Vesell ES. Assessment of aminopyrine metabolism in man by breath analysis after oral administration of 14C-aminopyrine. Effects of phenobarbital, disulfiram and portal cirrhosis. N Engl J Med 1974;291:1384-8.

3. Hepner GW, Vesell ES. Quantitative assessment of hepatic function by breath analysis after oral administration of (14C)aminopyrine. Ann Intern Med 1975;83:632-8.

4. Geboes KR, P. Verbeke K. . Ademtesten in de gastroenterologie. Tijdschr Gastroenterol 2003;41:14-6.

5. Irving CS, Schoeller DA, Nakamura KI, Baker AL, Klein PD. The aminopyrine breath test as a measure of liver function. A quantitative description of its metabolic basis in normal subjects. J Lab Clin Med 1982;100:356-73.

6. Baker AL, Kotake AN, Schoeller DA. Clinical utility of breath tests for the assessment of hepatic function. Semin Liver Dis 1983;3:318-29.

7. Dinesen L, Caspary WF, Chapman RW, Dietrich CF, Sarrazin C, Braden B. 13C-methacetin-breath test compared to also noninvasive biochemical blood tests in predicting hepatic fibrosis and cirrhosis in chronic hepatitis C. Dig Liver Dis 2008;40:743-8.

8. Petrolati A, Festi D, De Berardinis G, et al. 13C-methacetin breath test for monitoring hepatic function in cirrhotic patients before and after liver transplantation. Aliment Pharmacol Ther 2003;18:785-90.

9. Timbrell J. Principles of Biochemical Toxicology. Second ed. London: Taylor \& Francis; 1991.

10. Nelson DR, Koymans L, Kamataki T, et al. P450 superfamily: update on new sequences, gene mapping, accession numbers and nomenclature. Pharmacogenetics 1996;6:1-42.

11. Nebert DW. Suggestions for the nomenclature of human alleles: relevance to ecogenetics, pharmacogenetics and molecular epidemiology. Pharmacogenetics 2000;10:279-90.

12. van der Weide J, Steijns LS. Cytochrome P450 enzyme system: genetic polymorphisms and impact on clinical pharmacology. Ann Clin Biochem 1999;36 (Pt 6):722-9.

13. Anzenbacher P, Anzenbacherova E. Cytochromes P450 and metabolism of xenobiotics. Cell Mol Life Sci 2001;58:737-47.

14. Afolabi P, Wright $M$, Wootton SA, Jackson AA. Clinical utility of 13 C-liver-function breath tests for assessment of hepatic function. Dig Dis Sci 2013;58:33-41.

15. Schoeller DA, Kotake AN, Lambert GH, Krager PS, Baker AL. Comparison of the phenacetin and aminopyrine breath tests: effect of liver disease, inducers and cobaltous chloride. Hepatology 1985;5:276-81.

16. Brockmoller J, Roots I. Assessment of liver metabolic function. Clinical implications. Clin Pharmacokinet 1994;27:216-48.

17. Kajiwara M, Okazaki T, lida K, et al. Studies on 13C-phenacetin metabolism. II. A combination of breath test and urine test of in vivo metabolites in the diagnosis of liver disease. Chem Pharm Bull (Tokyo) 1996;44:1258-60.

18. Ciccocioppo R, Candelli M, Di Francesco D, et al. Study of liver function in healthy elderly subjects using the 13C-methacetin breath test. Aliment Pharmacol Ther 2003;17:271-7.

19. Braden B, Faust D, Sarrazin U, et al. 13C-methacetin breath test as liver function test in patients with chronic hepatitis $C$ virus infection. Aliment Pharmacol Ther 2005;21:179-85.

20. Holtmeier J, Leuschner M, Schneider A, Leuschner U, Caspary WF, Braden B. 13C-methacetin and 13Cgalactose breath tests can assess restricted liver function even in early stages of primary biliary cirrhosis. Scand J Gastroenterol 2006;41:1336-41.

21. Schneider A, Caspary WF, Saich R, et al. 13C-methacetin breath test shortened: 2-point-measurements after 15 minutes reliably indicate the presence of liver cirrhosis. J Clin Gastroenterol 2007;41:33-7.

22. Lara Baruque S, Razquin M, Jimenez I, Vazquez A, Gisbert JP, Pajares JM. 13C-phenylalanine and 13Cmethacetin breath test to evaluate functional capacity of hepatocyte in chronic liver disease. Dig Liver Dis 2000;32:226-32.

23. Razlan H, Marzuki NM, Tai ML, Shamsul AS, Ong TZ, Mahadeva S. Diagnostic value of the C methacetin breath test in various stages of chronic liver disease. Gastroenterol Res Pract 2011;2011:235796. 
24. Palmieri VO, Grattagliano I, Minerva F, Pollice S, Palasciano G, Portincasa P. Liver function as assessed by breath tests in patients with hepatocellular carcinoma. J Surg Res 2009;157:199-207.

25. Park GJ, Katelaris PH, Jones DB, Seow F, Le Couteur DG, Ngu MC. Validity of the 13C-caffeine breath test as a noninvasive, quantitative test of liver function. Hepatology 2003;38:1227-36.

26. Kotake AN, Schoeller DA, Lambert GH, Baker AL, Schaffer DD, Josephs H. The caffeine CO2 breath test: dose response and route of $\mathrm{N}$-demethylation in smokers and nonsmokers. Clin Pharmacol Ther 1982;32:261-9.

27. Park GJ, Katelaris $\mathrm{PH}$, Jones $\mathrm{DB}$, et al. The C-caffeine breath test distinguishes significant fibrosis in chronic hepatitis B and reflects response to lamivudine therapy. Aliment Pharmacol Ther 2005;22:395403.

28. Park GJ, Wiseman E, George J, et al. Non-invasive estimation of liver fibrosis in non-alcoholic fatty liver disease using the 13 C-caffeine breath test. J Gastroenterol Hepatol 2011;26:1411-6.

29. Brodie BB, Axelrod J. The fate of aminopyrine (pyramidon) in man and methods for the estimation of aminopyrine and its metabolites in biological material. J Pharmacol Exp Ther 1950;99:171-84.

30. Volz M, Kellner HM. Kinetics and metabolism of pyrazolones (propyphenazone, aminopyrine and dipyrone). Br J Clin Pharmacol 1980;10 Suppl 2:299S-308S.

31. Agundez JA, Martinez C, Benitez J. Metabolism of aminopyrine and derivatives in man: in vivo study of monomorphic and polymorphic metabolic pathways. Xenobiotica 1995;25:417-27.

32. Niwa T, Sato R, Yabusaki Y, Ishibashi F, Katagiri M. Contribution of human hepatic cytochrome P450s and steroidogenic CYP17 to the N-demethylation of aminopyrine. Xenobiotica 1999;29:187-93.

33. Noordhoek J, Dees J, Savenije-Chapel EM, Wilson JH. Output of $14 \mathrm{CO} 2$ in breath after oral administration of (14C-methyl) aminopyrine in hepatitis, cirrhosis and hepatic bilharziasis: its relationship to aminopyrine pharmacokinetics. Eur J Clin Pharmacol 1978;13:223-9.

34. Rodrigues $A D$. Integrated cytochrome P450 reaction phenotyping: attempting to bridge the gap between cDNA-expressed cytochromes P450 and native human liver microsomes. Biochem Pharmacol 1999;57:465-80.

35. Purba HS, Back DJ, Orme ML. Tolbutamide 4-hydroxylase activity of human liver microsomes: effect of inhibitors. Br J Clin Pharmacol 1987;24:230-4.

36. Iguchi S, Goromaru T, Noda A. Communications to the editor: A new metabolite of aminopyrine (aminophenazone) in man, 4-formylaminoantipyrine. Chem Pharm Bull (Tokyo) 1975;23:932-4.

37. Noda A, Goromaru T, Tsubone N, Matsuyama K, Iguchi S. In vivo formation of 4-formylaminoantipyrine as a new metabolite of aminopyrine. I. Chem Pharm Bull (Tokyo) 1976;24:1502-5.

38. Gradnik R, Fleischmann L. [Quantitative urinary excretion of various aminopyrine metabolites in man]. Pharm Acta Helv 1973;48:181-91.

39. Schneider AR, Teuber G, Paul K, et al. Patient age is a strong independent predictor of 13Caminopyrine breath test results: a comparative study with histology, duplex-Doppler and a laboratory index in patients with chronic hepatitis C virus infection. Clin Exp Pharmacol Physiol 2006;33:300-4.

40. Di Campli C, Angelini G, Armuzzi A, et al. Quantitative evaluation of liver function by the methionine and aminopyrine breath tests in the early stages of liver transplantation. Eur J Gastroenterol Hepatol 2003;15:727-32.

41. Candelli M, Armuzzi A, Nista EC, Fini L, Gasbarrini G, Gasbarrini A. 13C-methacetin breath test for monitoring hepatic function in cirrhotic patients before and after liver transplantation. Aliment Pharmacol Ther 2004;19:243.

42. Giannini E, Fasoli A, Chiarbonello B, et al. 13C-aminopyrine breath test to evaluate severity of disease in patients with chronic hepatitis $C$ virus infection. Aliment Pharmacol Ther 2002;16:717-25.

43. Caubet MS, Laplante A, Caille J, Brazier JL. [13C]aminopyrine and [13C]caffeine breath test: influence of gender, cigarette smoking and oral contraceptives intake. Isotopes Environ Health Stud 2002;38:717.

44. Ecochard $M$, Boillot $O$, Guillaud $O$, et al. Could metabolic liver function tests predict mortality on waiting list for liver transplantation? A study on 560 patients. Clin Transplant 2011;25:755-65.

45. Degre D, Bourgeois N, Boon N, et al. Aminopyrine breath test compared to the MELD and Child-Pugh scores for predicting mortality among cirrhotic patients awaiting liver transplantation. Transpl Int 2004;17:31-8. 
46. Forestier J, Dumortier J, Guillaud O, et al. Noninvasive diagnosis and prognosis of liver cirrhosis: a comparison of biological scores, elastometry, and metabolic liver function tests. Eur J Gastroenterol Hepatol 2010;22:532-40.

47. Giannini EG, Fasoli A, Borro P, et al. 13C-galactose breath test and 13C-aminopyrine breath test for the study of liver function in chronic liver disease. Clin Gastroenterol Hepatol 2005;3:279-85.

48. Braden B. Methods and functions: Breath tests. Best Pract Res Clin Gastroenterol 2009;23:337-52.

49. Kodaira $C$, Uchida S, Yamade $M$, et al. Influence of different proton pump inhibitors on activity of cytochrome P450 assessed by [(13)C]-aminopyrine breath test. J Clin Pharmacol 2012;52:432-9.

50. Wijnen PA, Op den Buijsch RA, Drent M, et al. Review article: The prevalence and clinical relevance of cytochrome P450 polymorphisms. Aliment Pharmacol Ther 2007;26 Suppl 2:211-9.

51. Flockhart DA. Drug interactions and the cytochrome P450 system. The role of cytochrome P450 2 C19. Clin Pharmacokinet 1995;29 Suppl 1:45-52.

52. Indiana University: P450 Drug Interaction Table. 2011. Available at http://medicine.iupui.edu/ clinpharm/ddis/main-table/. Accessed 22 August 2013. 


\section{Chapter 8}

A profile of volatile organic compounds in exhaled air as a potential non-invasive biomarker for

liver cirrhosis

K.E. Pijls*

A. Smolinska*

D.M.A.E. Jonkers

J.W. Dallinga

A.A.M. Masclee

G.H. Koek

F.J. van Schooten

*Both authors contributed equally to this work

Sci Rep 2016;6:19903 


\section{Abstract}

Early diagnosis of liver cirrhosis may prevent progression and development of complications. Liver biopsy is the current standard, but is invasive and associated with morbidity. Previously, non-invasive analysis of volatile organic compounds (VOCs) in breath could distinguish cirrhotic from healthy subjects. We aimed to identify an exhaled VOC profile within a heterogeneous group of chronic liver disease (CLD) patients that discriminates those with compensated cirrhosis (CIR) from those without cirrhosis, and to compare this with a panel of routine serological markers.

Breath samples were collected from 87 CLD and 34 CIR patients. VOCs in exhaled air were measured by gas chromatography time-of-flight mass spectrometry (GC-tof-MS). Partial Least Square Discriminant Analysis was performed to identify the optimal panel of serological markers and VOCs for classifying our patients using a random training set of $27 \mathrm{CIR}$ and 27 CLD patients. Two randomly selected independent internal validation sets and permutation test were used to validate the model.

A combination of 5 serological markers was found to distinguish CIR and CLD patients with a sensitivity of 0.71 and specificity of 0.84 . A set of 11 VOCs discriminated CIR from CLD patients with a sensitivity of 0.83 and specificity of 0.87 . Combining both did not further improve diagnostic accuracy.

In this feasibility study, we showed that a specific exhaled VOC profile can predict the presence of compensated cirrhosis among CLD patients with a higher accuracy than serological markers and thereby may aid in reducing liver biopsies. 


\section{Introduction}

Cirrhosis is an advanced stage of liver fibrosis accompanied by vascular remodeling. It is the end stage of chronic liver diseases, predominantly related to chronic viral infection, alcohol consumption, autoimmune and metabolic etiologies. Cirrhosis is usually characterized by an asymptomatic stage of compensated disease followed by a symptomatic stage of decompensated disease, defined by the presence of clinically evident complications. ${ }^{1}$ These complications, i.e. ascites, variceal hemorrhage, hepatic encephalopathy, and jaundice, are associated with a markedly reduced life expectancy. Early diagnosis of compensated cirrhosis may prevent development of severe complications, e.g. by endoscopic screening of or prophylactic treatment for gastrointestinal varices. ${ }^{2}$

Although a combination of imaging together with impaired liver synthetic function is nowadays considered useful in clinical practice, liver biopsy is still the standard for a definite diagnosis. ${ }^{3}$ Biopsy is, however, an invasive procedure associated with significant complications, ${ }^{4,5}$ and not suitable for follow-up. Also, liver biopsies are prone to sampling error and inter-observer variation. ${ }^{6,7}$

Many studies evaluated non-invasive alternatives to grade liver fibrosis, including serum biomarker panels and radiological tests. Transient elastography (i.e FibroScan), for example, accurately detected cirrhosis in various chronic liver diseases with area under the receiver operating characteristic curve (AUC) values of $0.87-0.99,{ }^{8}$ but its local availability differs. The aspartate aminotransferase (AST) to platelet ratio (APRI) has a reported AUC of 0.83 with a sensitivity of $76 \%$ and a specificity of $72 \%$ in a metaanalysis of 40 studies including HCV patients. ${ }^{9}$ FIB-4 is a serum biomarker panel that combines platelet count, alanine aminotransferase (ALT), AST and age and showed a high predictive accuracy for detecting cirrhosis (AUC 0.91) in HCV patients. ${ }^{10}$ These promising serum biomarker panels use parameters available from routine laboratory tests and estimate disease severity, but do not necessarily reflect the dynamic changes, which are currently considered to be involved in fibrogenesis, ${ }^{8}$ and/or hepatic metabolic function. Several breath tests using probe drugs as substrates have been applied to assess the functional metabolic capacity of the liver, but are easily affected by many factors, e.g. concomitant drug use and liver blood flow, especially in patients with chronic liver diseases. ${ }^{11,12}$

Analysis of volatile organic compounds (VOCs) in exhaled air was found to be useful for the diagnosis of several chronic diseases, including lung cancer, chronic inflammatory lung and intestinal diseases. ${ }^{13-17}$ As the liver plays a key role in metabolizing endogenous and exogenous substances, liver damage may lead to accumulation of metabolites in the systemic circulation that can be excreted in breath. ${ }^{18}$ Mainly pilot studies have identified exhaled VOCs to be associated with diseased liver. ${ }^{18-26}$ Specific combinations of VOCs discriminated between patients with cirrhosis and healthy controls. ${ }^{19-21,26}$ However, studies assessing the accuracy of exhaled VOCs in predicting 
the presence of cirrhosis within a heterogeneous group of chronic liver disease patients are still lacking.

Therefore, the primary aim of the present feasibility study was to identify an exhaled VOC profile for compensated liver cirrhosis that is able to discriminate chronic liver disease patients with from those without cirrhosis, independently from liver disease etiology and associated comorbidity, and to compare this with a panel of serological markers used in daily clinical practice.

\section{Materials and methods}

\section{Patients}

Consecutive patients with chronic liver diseases without (CLD, $n=87$ ) or with compensated cirrhosis ( $\mathrm{CIR}, n=34$ ) of different etiology and aged between 18-85 years were recruited from the Hepatology outpatient clinic between June 2010 and May 2012. Exclusion criteria were other known chronic gastrointestinal diseases (e.g. inflammatory bowel disease), chronic lung diseases (e.g. chronic obstructive lung disease, asthma, lung cancer) and rheumatoid arthritis (RA), or not willing to give informed consent.

Diagnosis of CLD and CIR was based on liver histology and/or clinical, laboratory, radiological and/or endoscopic findings. Compensated cirrhosis was defined by the absence of clinically evident complications (i.e. ascites, variceal hemorrhage, hepatic encephalopathy and/or jaundice). The severity of cirrhosis was assessed according to the Child-Pugh classification and Model for End Stage Liver Disease (MELD) score. The liver diagnosis was performed before exhaled air analysis. Serological markers as part of clinical care were analyzed routinely by the hospital's clinical chemistry laboratory and were retrieved from the computerized patient information system. The study population was a consecutive series of individuals defined by diagnostic and exclusion criteria.

Healthy volunteers ( $\mathrm{HC}, n=31$ ) were recruited via local advertisement and considered eligible for inclusion when having a normal medical history, physical examination and liver tests [alaline transaminase (ALT) and $\gamma$-glutamyl transpeptidase (GGT)]. Further exclusion criteria were a history of gastrointestinal, liver and/or lung diseases, RA and a history of excessive alcohol consumption (i.e. $>14$ beverages per week).

The study was approved by the Medical Ethics Committee of Maastricht University Medical Center (MUMC), conducted according to the revised version of the Declaration of Helsinki (October 2008, Seoul) and registered at the US National Library of Medicine (http://www.clinicaltrials.gov, NTC01081236 and NTC01279356). All subjects provided written informed consent prior to participation. 


\section{Sampling and analysis of exhaled air}

Breath samples were collected by inflating a 5L Tedlar bag (SKC Ltd, Dorset, UK) and then transferred by use of a vacuum pump (VWR International, France) to carbon-filled stainless steel sorption tubes (Markes International, Llantrisant Business Park, UK) to trap the VOCs within 1 hour. Thermal desorption GC-tof-MS was used to measure the breath samples, as previously described. ${ }^{13}$ The GC-tof-MS method applied here is a non-targeted GC-tof-MS method, i.e. before statistical analysis no prior identification of the compounds was performed.

\section{Data handling and statistical analyses}

Subject characteristics are presented as median (range) and were compared between groups using the Mann-Whitney $U$-test for non-parametric data. Dichotomous variables were compared with the $\chi^{2}$ test. A $P$ value $<0.05$ was considered statistically significant using a two-tailed test. Before the actual statistical analysis the raw chromatograms obtained by GC-tof-MS (called breathograms) of HC, CLD and CIR patients were preprocessed to diminish various sources of artefacts, such as noise (i.e. rapid vertical fluctuations in the baseline), column bleeding, and chromatographic drift. $^{27}$

Routinely tested serological markers (Table 8.1) and VOCs in exhaled air were investigated for their ability to distinguish between CLD and CIR patients. We used well-established Partial Least Square Discriminant Analysis (PLS-DA) with Significant Multivariate Correlation $(s M C)^{28}$ to identify serological markers or VOCs related to cirrhosis (Figure 8.1, step 3). ${ }^{27,29}$ PLS-DA is a well-established multivariate tool commonly used in the field of metabolomics as well as in biomarker research. ${ }^{30}$ PLS-DA constructs and optimizes a linear classification model based on the covariance between the serological markers or VOCs (the variables) and the final diagnosis (CIR or CLD), with optimal discrimination between the patient groups. The PLS-DA can be defined as a two equations model, ${ }^{31}$ for data matrix $X$ and corresponding vector of class membership $y: X=T P+E(1), y=T q+f(2)$, where $T$ is a score matrix containing orthogonal latent variables in the columns, ${ }^{28} E$ and $f$ are model residuals, $P$ is $X$ loading while $q$ is $Y$ loading. The relation between $X$ and $y$ can be expressed as: $y=X b+r(3)$, where $b$ is a vector of regression coefficient and $r$ a vector of model residuals. The regression coefficient reflects the relative importance of the variables (here VOCs or serological markers) in predicting cirrhosis among CLD patients. Score matrix $T$ will be used for the PLS-DA score plot. The combination of score matrix $T$ and loading matrix $P$ are used for PLS-DA bi-plot. The PLS-DA was applied on a training set of randomly selected $27 \mathrm{CIR}$ and 27 CLD patients (Figure 8.1, step 2). The prediction performance was established using a validation set containing $7 \mathrm{CLD}$ and $7 \mathrm{CIR}$ patients (randomly selected from the whole population, repeated 1000 times, i.e. validation set 1$).^{32}$ For selecting training set and validation set 1 , repeated stratified holdout (or stratified 
random subsampling) approach was used. ${ }^{33}$ Finally, the significance of PLS-DA classification models was evaluated by the permutation test with 1000 iterations. $^{32}$

The prediction accuracy of the PLS-DA classification models was graphically represented using the Receiver Operating Characteristic (ROC) curve, using group probabilities obtained from PLS-DA analysis. The probabilities for each sample add up by definition to 1 (e.g. 0.8 for CIR and 0.2 for CLD). The probabilities can be considered as a range of cut-off for which individual sensitivities and specificities are calculated and represented in the ROC curve. The optimal cut-off is used (here 0.505) to obtain the sensitivity and specificity of the PLS-DA model. The area under the curve (AUC) of the ROC curve is an indicator of predictive performance: a value close to 1 indicates high predictive power of the classification model. The prediction accuracy was also calculated for the remaining 53 CLD patients (i.e. validation set 2).

The outcome of the PLS-DA VOC classification model was visualized using a score plot and bi-plot. The score plot demonstrates the relations (i.e. similarity) between all patients. Each individual is represented as a single point. Those points that lie close to each other have similar properties (i.e. VOC profile), while points that are far away have different properties. In the bi-plot, information on the correlation between compounds and differences in relative abundance between CLD and CIR patients are presented.

Table 8.1 Characteristics of patients.

\begin{tabular}{lccc}
\hline & $\begin{array}{c}\text { CIR patients } \\
(\mathrm{n}=34)\end{array}$ & $\begin{array}{c}\text { CLD patients } \\
(\mathrm{n}=87)\end{array}$ & $P$ value \\
\hline Age (years) & $59.5(18-74)$ & $54(24-75)$ & 0.030 \\
Sex $(\mathrm{M} \mathrm{/} \mathrm{F)}$ & $21 / 13$ & $47 / 40$ & 0.440 \\
BMI $\left(\mathrm{kg} / \mathrm{m}^{2}\right)$ & $25.3(18.7-44.6)$ & $28.3(18.7-48.4)$ & 0.037 \\
Smokers & 12 & 20 & 0.191 \\
AF $(\mathrm{U} / \mathrm{L})$ & $121(46-230)$ & $110(58-680)$ & 0.630 \\
GGT (U/L) & $54.5(10-370)$ & $69(16-1337)$ & 0.130 \\
AST (U/L) & $41(13-135)$ & $35(11-487)$ & 0.483 \\
ALT (U/L) & $28.5(9-199)$ & $46(13-231)$ & 0.005 \\
Bilirubin $(\mu \mathrm{mol} / \mathrm{L})$ & $18.9(9-62.9)$ & $13.4(6.4-152.3)(n=84)$ & 0.000 \\
Albumin $(\mathrm{g} / \mathrm{L})$ & $37.4(26.1-46.3)$ & $40.2(23.9-46.5)(n=71)$ & 0.006 \\
Creatinine $(\mu \mathrm{mol} / \mathrm{L})$ & $74(40-108)$ & $72.5(13-113)(n=80)$ & 0.985 \\
Thrombocytes x10 $/ \mathrm{L}$ & $112.5(43-441)$ & $233(99-401)(n=84)$ & 0.000 \\
Child-Pugh score* & $5(5-9)$ & - & - \\
MELD-score & $8(6-15)$ & - & - \\
Liver histology & 24 & 47 & - \\
\hline
\end{tabular}

Continuous values are presented as medians (range). * One patient had a Child-Pugh score of 9, due to high bilirubin levels because of Gilbert's Syndrome. None of the patients had clinically evident ascites, variceal hemorrhage, hepatic encephalopathy and/or jaundice. 


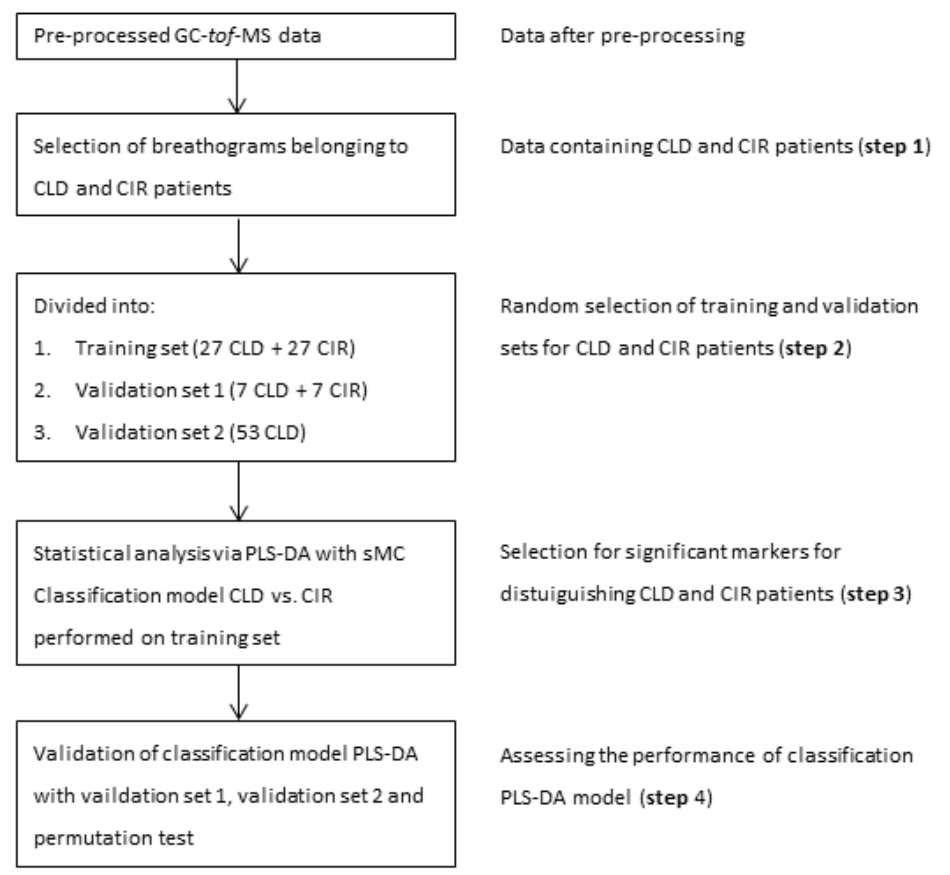

Figure 8.1 The conceptual flow chart of the statistical analysis. Steps 2 to 4 apply for serological markers and VOCs.

\section{Results}

\section{Patients}

Exhaled air was straightforwardly and safely obtained from all patients and HC. From the total of 121 patients, none was excluded from the analysis. No significant differences were observed for gender or smoking between both patients groups (Table 8.1). However, CIR patients were significantly older $(P=0.030)$, and had a lower $\mathrm{BMI}$ $(P=0.037)$ than CLD patients.

The etiology of the $34 \mathrm{CIR}$ patients was alcohol-related $(n=9)$, chronic viral infection $(n=1)$, autoimmune-related $(n=7)$, metabolic $(n=4)$ and multifactorial or undefined in 13 patients. The Child-Pugh class of the cirrhotic patients was A in 24 and B in 10 patients, and the median international normalized ratio (INR) was 1.11 (0.95-1.51).

The etiology of the 87 CLD patients was metabolic $(n=28)$, autoimmune-related $(n=25)$, chronic active viral infection $(n=16)$, alcohol-related $(n=3)$ and multifactorial or undefined in 15 patients.

All $31 \mathrm{HC}$ (52\% male), with a median age of 47 (18-78) years and median BMI of 24.7 $(18.1-32.1) \mathrm{kg} / \mathrm{m}^{2}$ had normal liver tests [GGT $18(6-50) \mathrm{U} / \mathrm{I}$ and ALT $\left.18(10-32) \mathrm{U} / \mathrm{I}\right]$. 
Drug therapy as standard medical care was given to all patients, including among others antibiotics $(n=3)$, glucocorticosteroids $(n=7)$, immunosuppressives $(n=9)$, laxatives $(n=11)$, proton pump inhibitors (PPIs) $(n=43)$ and ursodeoxycholic acid $(n=53)$.

\section{Serological markers}

Liver functional mass was more impaired in CIR compared to CLD patients as shown by significantly lower ALT, GGT and albumin levels, and increased bilirubin levels (Table 8.1). The decrease of thrombocytes in cirrhotic patients may be the result of hypersplenism, which is indicative for the presence of portal hypertension. A combination of 5 markers, i.e. GGT, ALT, bilirubin, albumin, and thrombocytes, was the most discriminatory between CLD and CIR with a sensitivity and specificity of 0.71 and 0.84, respectively, and an AUC of 0.81 (95\% confidence interval: 0.77-0.91) (Figure 8.2A). Four of these serological markers were also statistically significant (Table 8.1). The permutation test using 1000 iterations led to a $P$ value of 0.001 . The prediction of the 53 remaining CLD patients was $84.40 \%$.

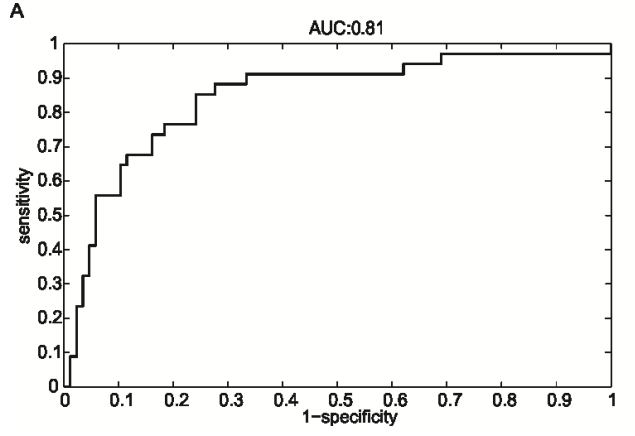

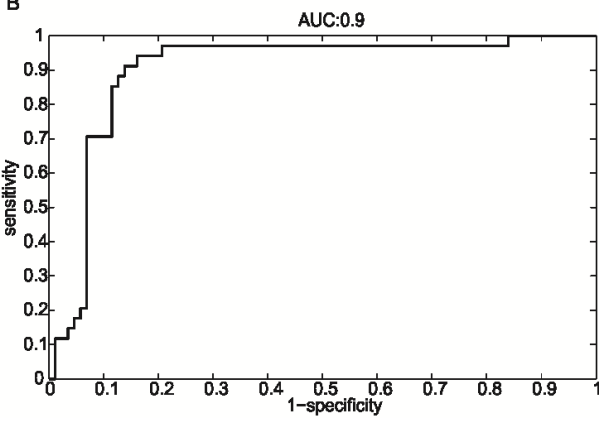

Figure 8.2 ROC curve for validation set 1 of the PLS-DA classification model obtained for CLD and CIR patients using, A: 5 selected serological markers, AUC: 0.81 ( $95 \%$ confidence interval 0.77-0.91); B: 11 discriminatory VOCs, AUC: 0.90 (95\% confidence interval 0.86-0.96)

\section{VOCs}

In total, 152 GC-tof-MS breathograms were measured (34 CIR, $87 \mathrm{CLD}$ and $31 \mathrm{HC}$ ) and preprocessed, ${ }^{27}$ resulting in a data matrix containing 3718 compounds (i.e. individual VOCs).

As proof of principle and to confirm previous publications, ${ }^{19-21,26}$ we compared breathograms of $\mathrm{HC}$ with those of CLD and CIR patients. A set of 23 and 19 compounds enabled differentiating HC from CLD and CIR patients, with a correct classification of $100 \%$ and $93.75 \%$, respectively (data not shown).

A VOC profile based on 11 compounds was identified using PLS-DA and allowed discriminating CIR from CLD patients. This PLS-DA model was then applied to the validation set 1 , which contained; 7 CLD and 7 CIR. The corresponding ROC analysis 
showed sensitivity of 0.83 , specificity of 0.87 and AUC of 0.90 (95\% confidence intervals; 0.86-0.96) for the diagnosis of cirrhosis (Figure 8.2B). The averaged negative (NPV), and positive predictive values (PPV) for CIR patients were $84.14 \%$ and $85.30 \%$, respectively. The prediction of validation set 2, i.e. comprising the 53 remaining breathograms of CLD patients, was $86.80 \%$.

The PLS-DA score plot for the VOCs is shown in Figure 8.3. Here, two new variables (LV1 and LV2) were used to represent a linear combination of 11 discriminatory VOCs, showing the proportion of each VOC in discriminating between CLD and CIR patients. Each point represents a single patient, is color-coded for group membership (i.e. CLD and $(\mathrm{R}$ ) and marked as training or validation set. The samples of CLD and CIR patients of validation set 1 and 2 are projected accurately, i.e. within the cloud of the training samples belonging to the matching group. To further validate the obtained classification PLS-DA model, a permutation test was carried out using 1000 iterations and resulted in a $P$ value of 0.002 .

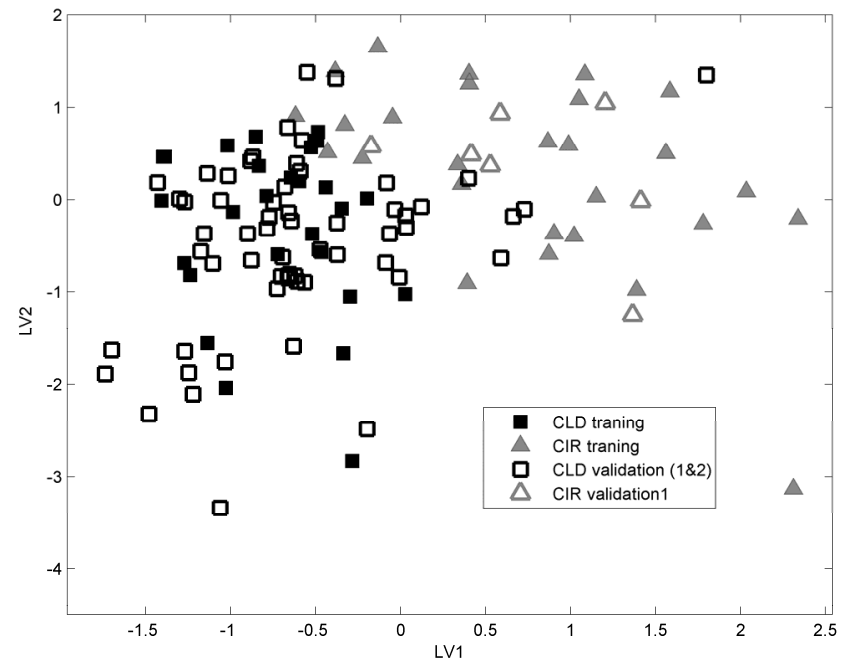

Figure 8.3 PLS-DA score plot of the final classification model obtained for CLD and CIR patients. Each patient is represented as a point (black filled square for training samples of CLD patients, grey filled triangle for training samples of CIR patients, black square for validation set 1 and validation set 2 of CLD patients, and grey triangle for validation set 1 of CIR patients). The two patient groups form distinct clusters indicating differences in VOCs profile.

Finally, to further test the significance of the set of 11 VOCs regularized Multivariate Analysis of Variance (rMANOVA) ${ }^{34}$ showed to be statistically significant $(P<0.001)$. The effect of etiology, drug therapy (i.e. antibiotics, glucocorticosteroids, immunosuppressive, laxatives, PPIs and ursodeoxycholic acid) and smoking in CLD and CIR patients on the selected set of discriminatory VOCs was also investigated using 
rMANOVA. No significant changes were found in selected VOCs with respect to etiology $(P=0.48)$, drug therapy $(P=0.23)$ and smoking $(P=0.12)$. The influence of alcohol could not be investigated, since only 3 patients were consuming alcoholic beverages at time of the study.

\section{Identification and relative abundance of VOCs}

The 11 discriminatory VOCs were chemically identified by spectrum recognition using The National Institute of Standards and Technology library in combination with spectrum interpretation by an experienced mass-spectrometrist and identification based on retention times of compounds.

The PLS-DA bi-plot (Figure 8.4) shows the change of compounds relative abundance between CLD and CIR patients. Each point corresponds to a patient, while the line corresponds to a compound. Correlation between compounds is reflected by the angle between the lines. For example, octane (VOC3) and propionic acid (VOC2) are positively correlated and their amounts are elevated in CIR compared to CLD patients. A negative correlation is found for propionic acid (VOC2) and 1-hexadecanol (VOC9) and the absence of correlation is found, for instance, between 3-carene (VOC6) and 1hexadecanol (VOC9). The chemical identity of the discriminatory compounds and information about changes in relative abundances are listed in Table 8.2.

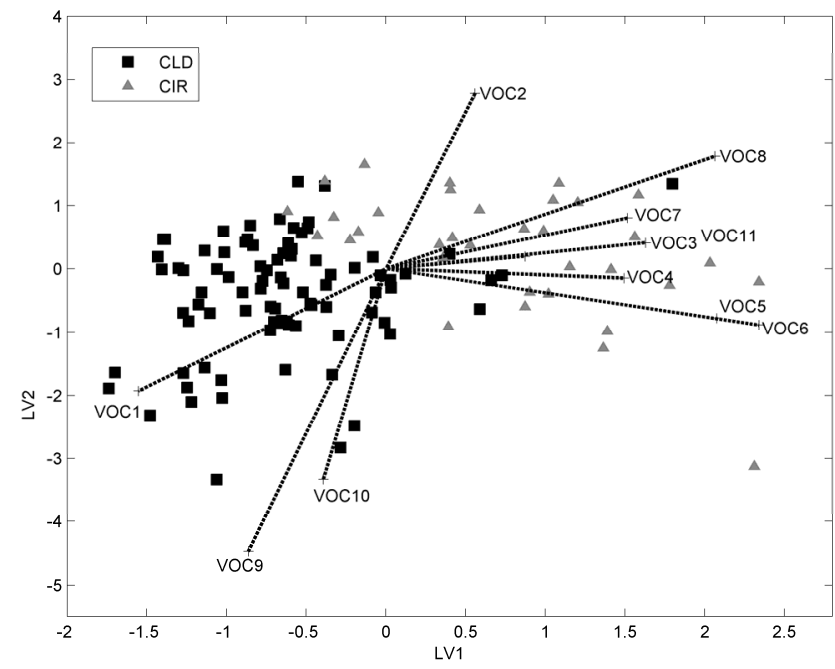

Figure 8.4 Bi-plot of PLS-DA analysis performed on 11 discriminatory VOCs in the breath of CLD ( $n=87)$ and CIR patients $(n=34)$. Every point corresponds to a single breath sample and lines to the VOCs. VOC2 to VOC8 and VOC11 are found to be elevated in CIR patients. VOC1, VOC9, and VOC10 are reduced in CIR patients. 
Table 8.2 The identification of 11 discriminating VOCs.

\begin{tabular}{lll}
\hline $\mathrm{Nr}$. & Chemical identity & Change \\
\hline 1 & 3-methylbutanal & $(-)$ \\
2 & Propanoic acid & $(+)$ \\
3 & Octane & $(+)$ \\
4 & Terpene $(\mathrm{C} 10 \mathrm{H} 16)$ & $(+)$ \\
5 & Terpenoid: $\alpha$-pinene & $(+)$ \\
6 & 3-carene & $(+)$ \\
7 & Unknown & $(+)$ \\
8 & Branched C16H34 & $(+)$ \\
9 & 1-hexadecanol & $(-)$ \\
10 & Branched C16H34 & $(-)$ \\
11 & Dimethyl disulfide & $(+)$ \\
\hline
\end{tabular}

$(+)$ indicates elevation and (-) indicates reduction in relative abundance of a compound in CIR compared to CLD patients.

\section{Discussion}

This study demonstrated that a combination of serological markers, i.e. GGT, ALT, bilirubin, albumin, and thrombocytes, can discriminate cirrhotic patients from patients with chronic liver diseases with a sensitivity of 0.71 and specificity of 0.84 . A profile of 11 VOCs was found to predict the presence of cirrhosis with a sensitivity of 0.83 and a specificity of 0.87 . The combination of both did not improve the diagnostic accuracy. In our study we used GC-tof-MS to measure volatiles in exhaled air. This technique was combined with multivariate analysis based on established classification method PLSDA. Next to GC-tof-MS, other analytical techniques can be used to measure various volatiles in exhaled air for instance Ion Mobility Spectrometry, ${ }^{35}$ Proton Transfer Reaction - Mass Spectrometry ${ }^{36}$ or Selected Ion-Flow Tube Mass-Spectrometry. ${ }^{14}$ All these analytical techniques have their advantages and disadvantages described elsewhere. $^{37,38}$ However, all can be combined with various machine learning techniques, such as PLS-DA, decision trees, Random Forests and Support Vector Machine, to identify most discriminatory patterns of compounds between cases and controls. ${ }^{39,40}$ The choice depends on the characteristics of the data set. In the current study, optimal discrimination could be found by PLS-DA. However, independently from the applied statistical technique, the objective is always to unravel important information in the data.

To our knowledge, this is the first study that applied VOC analysis to predict the presence of compensated liver cirrhosis among CLD patients with different etiologies. We used an approach that analyzed the entire range of VOCs present in exhaled air. The classification model using 11 VOCs was very accurate in discriminating between CLD and CIR patients. Although, the numbers in the first validation set were small (7 versus 7 , respectively), findings were further confirmed by correctly classifying $86.80 \%$ of the remaining 53 CLD patients in the validation set 2 . Additionally, the 
permutation test was used to endorse the findings, indicating only $0.2 \%$ chance that the difference between groups was made by chance.

Our study confirmed that serological markers have a rather good diagnostic accuracy to detect liver cirrhosis. ${ }^{8}$ Although, their specificity was comparable to VOCs, VOCs revealed a better sensitivity.

Half of the significant compounds appear to be hydrocarbons, including linear and branched alkanes, alkenes and aromatic compounds, which might be related to oxidative stress and/or an impaired metabolism by cytochrome P450 enzymes in the liver. ${ }^{20}$ Furthermore, we found two different terpenes levels $\left(\mathrm{C}_{10} \mathrm{H}_{16}\right.$ and terpenoid: $\alpha$ pinene) increased in CIR compared to CLD patients, consistently with other studies. ${ }^{20,21}$ An impaired liver metabolism could be involved as terpenes are metabolized by cytochrome P450 enzymes. ${ }^{20}$ Similarly to previous studies, we found higher level of dimethyl disulfide in CIR patients, which may be generated by incomplete metabolism of methionine in the transamination pathway. ${ }^{20,41-43}$

In our population, cirrhosis was caused by alcohol in about $25 \%$ of patients and only 3 individuals still consumed alcohol at the moment of investigations. Thereby the direct toxic and metabolic effect of alcohol on the obtained results in this population is very unlikely. This is further supported by the lack of compounds identified related to alcohol consumption. A subgroup of patients was diagnosed with non-alcoholic fatty liver disease (NAFLD). Using a similar approach, Verdam et al., ${ }^{22}$ found n-tridecane, 3-methyl-butanonitrile, and 1-propanol to discriminate overweight subjects with from those without non-alcoholic steatohepatitis (NASH). These compounds were not discriminatory in the present study, probably because of different populations included.

Not all discriminatory VOCs could be associated with pathological processes related to the liver. However, evidence is emerging that changes in the intestinal microbiota do occur in patients with liver diseases and their differential metabolic activity may be detected as volatile compounds.

In our study we tried to predict the presence of cirrhosis among a heterogeneous group of chronic liver disease patients, while in the majority of published studies patients with liver cirrhosis were compared to healthy controls. We also compared the VOCs from HC with the two patient groups, i.e. chronic liver disease with or without cirrhosis. Subsets of 23 and 19 compounds were able to differentiate CLD and CIR patients from $\mathrm{HC}$ with a correct classification of $100 \%$ and $93.75 \%$ in an independent validation set, thereby confirming previous studies. ${ }^{19-21,26,36}$ The lack of overlap between these subsets and the 11 compounds discriminating between CLD and CIR indicates that in order to find sensitive and specific diagnostic markers for cirrhosis in patients with chronic liver diseases, profiles should be compared between CIR and CLD patients.

Liver biopsies were obtained in $70 \%$ of the patients. In the remaining patients cirrhosis was confirmed by evident clinical, laboratory, radiological and/or endoscopic findings, which is in line with current expert opinion of diagnosing cirrhosis. ${ }^{3}$ Also, it has to be 
noted that some baseline characteristics differed between both patient groups. The higher age in CIR patients can be explained by disease progression and is inevitable when studying a representative sample from daily clinical practice. The lower BMI may be related to malnutrition and muscle wasting, often present in CIR patients. ${ }^{44,45}$ Another drawback is that not all discriminatory VOCs have full chemical identification. Although the chemical identification gives leads towards pathophysiological pathways, this was not the aim of the study and does require a different study design. Furthermore, a large variety of VOCs are present in environmental air, but is not expected to be an important factor since all breath samples were taken randomly in time and at the same location.

The discriminating VOCs were also not affected by etiology, drug therapy and smoking as tested by rMANOVA. The effect of diet was not tested, as this information was not available for this cohort from daily clinical practice, and previous analysis could not find any relation between discriminatory VOCs and diet. ${ }^{46,47}$ It should be noted that we deliberately included a heterogeneous group of patients to prove the concept that VOCs can be used to diagnose cirrhosis among patients with CLD independently of etiology, drug therapy and without standardization of dietary intake.

We investigated 121 patients with CLD. Other studies investigating liver cirrhosis with exhaled breath analysis usually included $60-70$ individuals. ${ }^{21,22,25,36}$ To strengthen our findings, we used an independent validation set as well as permutation test. ${ }^{32}$ Although the sample size in this proof-of-concept study was reasonable, findings should be further validated using a large multicenter cohort with well-diagnosed patients. Moreover, it would be interesting to investigate the potential of VOCs to assess disease progression in prospective cohorts, as well as to study subgroups of patients with cirrhosis associated with complications or different stages of fibrosis, and to compare it for example with transient elastography.

VOCs can reflect liver metabolic function and, therefore, may provide leads towards pathophysiological pathways. Additionally, VOCs have a high short-term reproducibility ${ }^{48}$ and exhaled air collection is non-invasive, easy and requires only a minimal time investment. This technique is suitable to use in large cohorts, and could be implemented in clinical practice using sensor analysis. Much progress has been made in miniaturizing instruments that ultimately lead to manufacturing easy-to-use, cheap, hand-held equipment, in varying forms of sensors to detect volatile chemicals. Recently the electronic nose (e-Nose) has been shown to be of value in detecting acute liver failure in rats. ${ }^{49}$ In humans, the e-Nose has already proven efficacy in other fields such as asthma and chronic obstructive lung disease. ${ }^{50,51}$

In conclusion, our findings indicate that the analysis of exhaled VOCs can more accurately predict the presence of compensated liver cirrhosis among CLD patients with different etiology than a panel of routine serological markers. Although the sensitivity and specificity should be further improved, the NPV of $84.10 \%$ indicates that VOCs may aid in reducing the number of liver biopsies in clinical practice. 


\section{References}

1. Garcia-Tsao G, Friedman S, Iredale J, Pinzani M. Now there are many (stages) where before there was one: In search of a pathophysiological classification of cirrhosis. Hepatology 2010;51:1445-9.

2. Garcia-Tsao G, Lim JK, Members of Veterans Affairs Hepatitis CRCP. Management and treatment of patients with cirrhosis and portal hypertension: recommendations from the Department of Veterans Affairs Hepatitis C Resource Center Program and the National Hepatitis C Program. Am J Gastroenterol 2009;104:1802-29.

3. Tsochatzis EA, Bosch J, Burroughs AK. Liver cirrhosis. Lancet 2014;383:1749-61.

4. Rockey DC, Caldwell SH, Goodman ZD, Nelson RC, Smith AD. Liver biopsy. Hepatology 2009;49:101744.

5. Myers RP, Fong A, Shaheen AA. Utilization rates, complications and costs of percutaneous liver biopsy: a population-based study including 4275 biopsies. Liver international : official journal of the International Association for the Study of the Liver 2008;28:705-12.

6. Bedossa P, Dargere D, Paradis V. Sampling variability of liver fibrosis in chronic hepatitis C. Hepatology 2003;38:1449-57.

7. Ratziu V, Charlotte F, Heurtier A, et al. Sampling variability of liver biopsy in nonalcoholic fatty liver disease. Gastroenterology 2005;128:1898-906.

8. Patel K, Bedossa P, Castera L. Diagnosis of liver fibrosis: present and future. Semin Liver Dis 2015;35:166-83.

9. Lin ZH, Xin YN, Dong QJ, et al. Performance of the aspartate aminotransferase-to-platelet ratio index for the staging of hepatitis C-related fibrosis: an updated meta-analysis. Hepatology 2011;53:726-36.

10. Vallet-Pichard A, Mallet V, Nalpas B, et al. FIB-4: an inexpensive and accurate marker of fibrosis in HCV infection. comparison with liver biopsy and fibrotest. Hepatology 2007;46:32-6.

11. Afolabi $P$, Wright $M$, Wootton SA, Jackson AA. Clinical utility of 13 C-liver-function breath tests for assessment of hepatic function. Dig Dis Sci 2013;58:33-41.

12. Pijls KE, de Vries H, Nikkessen S, Bast A, Wodzig WK, Koek GH. Critical appraisal of $13 \mathrm{C}$ breath tests for microsomal liver function: aminopyrine revisited. Liver Int 2014;34:487-94.

13. Smolinska A, Klaassen EM, Dallinga JW, et al. Profiling of volatile organic compounds in exhaled breath as a strategy to find early predictive signatures of asthma in children. PLoS One 2014;9:e95668.

14. Bodelier AGL, Smolinska A, Baranska A, et al. Volatile Organic Compounds in Exhaled Air as Novel Marker for Disease Activity in Crohn's Disease: A Metabolomic Approach. IBD 2015;21:1776-85.

15. Wang $C S$, Dong $R$, Wang $X Y$, et al. Exhaled volatile organic compounds as lung cancer biomarkers during one-lung ventilation. Sci Rep 2014;4.

16. Broza YY, Zuri L, Haick H. Combined Volatolomics for Monitoring of Human Body Chemistry. Sci Rep 2014;4.

17. Lavra L, Catini A, Ulivieri A, et al. Investigation of VOCs associated with different characteristics of breast cancer cells. Sci Rep 2015;5.

18. Khalid T, Richardson P, Probert CS. The liver breath! Breath volatile organic compounds for the diagnosis of liver disease. Clin Gastroenterol Hepatol 2014;12:524-6.

19. Netzer M, Millonig G, OsI M, et al. A new ensemble-based algorithm for identifying breath gas marker candidates in liver disease using ion molecule reaction mass spectrometry. Bioinformatics 2009;25:941-7.

20. Dadamio J, Van den Velde S, Laleman W, et al. Breath biomarkers of liver cirrhosis. J Chromatogr B: Anal Technol Biomed Life Sci 2012;905:17-22.

21. Morisco F, Aprea E, Lembo V, et al. Rapid "breath-print" of liver cirrhosis by proton transfer reaction time-of-flight mass spectrometry. A pilot study. PLoS One 2013;8:e59658.

22. Verdam FJ, Dallinga JW, Driessen A, et al. Non-alcoholic steatohepatitis: a non-invasive diagnosis by analysis of exhaled breath. J Hepatol 2013;58:543-8.

23. Sehnert SS, Jiang L, Burdick JF, Risby TH. Breath biomarkers for detection of human liver diseases: preliminary study. Biomarkers : biochemical indicators of exposure, response, and susceptibility to chemicals 2002;7:174-87. 
24. Solga SF, Alkhuraishe A, Cope K, et al. Breath biomarkers and non-alcoholic fatty liver disease: preliminary observations. Biomarkers : biochemical indicators of exposure, response, and susceptibility to chemicals 2006;11:174-83.

25. Millonig G, Praun S, Netzer M, et al. Non-invasive diagnosis of liver diseases by breath analysis using an optimized ion-molecule reaction-mass spectrometry approach: a pilot study. Biomarkers : biochemical indicators of exposure, response, and susceptibility to chemicals 2010;15:297-306.

26. Van den Velde S, Nevens F, Van Hee P, van Steenberghe D, Quirynen M. GC-MS analysis of breath odor compounds in liver patients. Journal of chromatography B, Analytical technologies in the biomedical and life sciences 2008;875:344-8.

27. Smolinska A, Hauschild AC, Fijten RR, Dallinga JW, Baumbach J, van Schooten FJ. Current breathomicsa review on data pre-processing techniques and machine learning in metabolomics breath analysis. Journal of breath research 2014;8:027105.

28. Afanador NL, Tran TN, Blanchet L, Buydens LMC. Variable importance in PLS in the presence of autocorrelated data - Case studies in manufacturing processes. Chemom Intell Lab Syst 2014;139:13945.

29. Gidskehaug L, Anderssen E, Alsberg BK. Cross model validation and optimisation of bilinear regression models. Chemom Intell Lab Syst 2008;93:1-10.

30. Lindon J, Nicholson J, Holmes E. The Handbook of Metabonomics and Metabolomics: Elseview; 2006.

31. Brereton RG, Lloyd GR. Partial least squares discriminant analysis: taking the magic away. J Chemom 2014;28:213-25.

32. Westerhuis JA, Hoefsloot HCJ, Smit S, et al. Assessment of PLSDA cross validation. Metabolomics 2008;4:81-9.

33. Simon R. Resampling Strategies for Model Assessment and Selection. In: Dubitzky W, Granzow M, Berrar D, eds. Fundamentals of Data Mining in Genomics and Proteomics: Springer US; 2008.

34. Engel J, Blanchet L, Bloemen B, et al. Regularized MANOVA (rMANOVA) in untargeted metabolomics. Anal Chim Acta 2015;899:1-12.

35. Baumbach J, Bunkowski A, Lange S, et al. IMS2- An integrated medical software system for early lung cancer detection using ion mobility spectrometry data of human breath. J Integr Bioinform 2007;4:75.

36. Fernandez del Rio R, O'Hara ME, Holt A, et al. Volatile Biomarkers in Breath Associated With Liver Cirrhosis - Comparisons of Pre- and Post-liver Transplant Breath Samples EBioMedicine 2015;2: 1243-50.

37. Schneider T, Hauschild AC, Baumbach JI, Baumbach J. An integrative clinical database and diagnostics platform for biomarker identification and analysis in ion mobility spectra of human exhaled air. J Integr Bioinform 2013;10:218.

38. Boots AW, van Berkel JJBN, Dallinga JW, Smolinska A, Wouters EF, van Schooten FJ. The versatile use of exhaled volatile organic compounds in human health and disease. J Breath Res 2012;6.

39. Hauschild AC, Baumbach JI, Baumbach J. Integrated statistical learning of metabolic ion mobility spectrometry profiles for pulmonary disease identification. Genet Mol Res 2012;11:2733-44.

40. Hauschild AC, Frisch T, Baumbach JI, Baumbach J. Carotta: Revealing Hidden Confounder Markers in Metabolic Breath Profiles. Metabolites 2015;5:344-63.

41. Tangerman A, Meuwese-Arends MT, Jansen JB. Cause and composition of foetor hepaticus. Lancet 1994;343:483.

42. Chen S, Mahadevan V, Zieve L. Volatile fatty acids in the breath of patients with cirrhosis of the liver. J Lab Clin Med 1970;75:622-7.

43. Kaji H, Hisamura M, Saito N, Murao M. Evaluation of volatile sulfur compounds in the expired alveolar gas in patients with liver cirrhosis. Clin Chim Acta 1978;85:279-84.

44. Montano-Loza AJ. Muscle wasting: a nutritional criterion to prioritize patients for liver transplantation. Curr Opin Clin Nutr Metab Care 2014;17:219-25.

45. Montano-Loza AJ, Meza-Junco J, Prado CM, et al. Muscle wasting is associated with mortality in patients with cirrhosis. Clin Gastroenterol Hepatol 2012;10:166-73, 73 e1.

46. Tigchelaar EF, Zhernakova A, Dekens JAM, et al. An introduction to Lifelines DEEP: study design and baseline characteristics. BMJ Open 2015;5:e006772.

47. Baranska A, Tigchelaar E, Smolinska A, et al. Profile of volatile organic compounds in exhaled breath changes as a result of gluten-free diet. J Breath Res 2013;7:037104. 
48. Robroeks CM, van Berkel JJ, Dallinga JW, et al. Metabolomics of volatile organic compounds in cystic fibrosis patients and controls. Pediatr Res 2010;68:75-80.

49. Wlodzimirow KA, Abu-Hanna A, Schultz MJ, et al. Exhaled breath analysis with electronic nose technology for detection of acute liver failure in rats. Biosens Bioelectron 2013;53C:129-34.

50. Fens N, Zwinderman AH, van der Schee MP, et al. Exhaled breath profiling enables discrimination of chronic obstructive pulmonary disease and asthma. Am J Respir Crit Care Med 2009;180:1076-82.

51. Incalzi RA, Pennazza G, Scarlata S, et al. Reproducibility and respiratory function correlates of exhaled breath fingerprint in chronic obstructive pulmonary disease. PLoS One 2012;7:e45396. 
Chapter 9

General discussion 


\section{General discussion}

Cirrhosis is the end stage of chronic liver diseases with alcohol consumption, viral infections and metabolic disorders as predominant causes. Recent studies indicate that apart from these etiological factors causing liver damage, the intestine is an important player in the pathophysiology of chronic liver diseases, cirrhosis and cirrhosis-related complications. This thesis aims to get further insight into the pathophysiology and development of non-invasive diagnostics for chronic liver diseases, especially cirrhosis at an early stage, as this is important to prevent progression towards end stage, complicated, liver disease.

\section{The role of the intestine}

\section{The role of the intestine in cirrhosis}

Enhanced translocation of luminal bacteria and their products through the intestinal barrier into the portal and systemic circulation is an accepted mechanism that contributes to liver damage and development of cirrhosis-related complications. The intestinal barrier is essential to prevent enhanced bacterial translocation via both transcellular and paracellular pathways. ${ }^{1}$ In this thesis, we focused on the paracellular permeability regulated by tight junctions (TJs) and adherens junctions (AJs) in patients with compensated liver cirrhosis, as an increased permeability might be a risk factor for the development of complications that, to a large extent, are responsible for the high morbidity and mortality (Chapter 3 ). Paracellular permeability was assessed by a multi-sugar test and by the expression of TJ genes and proteins in duodenal and sigmoid biopsies of compensated cirrhotic patients and compared with age, gender and BMI-matched healthy controls. Gastroduodenal and small intestinal permeability were found to be comparable between both groups. Previous studies did provide evidence of an increased gastroduodenal and small intestinal permeability, but were mainly performed in mixed groups of patients with compensated and decompensated cirrhosis. ${ }^{2}$ These studies also indicated that changes in intestinal permeability are more pronounced in patients with advanced cirrhosis, especially in those with complications (Chapter 2). However, when we analyzed small intestinal permeability in more detail, we observed an increased permeability in a subgroup of alcohol-related cirrhotic patients. This finding confirms the pronounced and directly disruptive effects of alcohol and its non-oxidative metabolites on the epithelial barrier that have been demonstrated in both in vitro and in vivo animal and human studies. ${ }^{3}$ Remarkably, we observed an increased permeability of the large intestine in the whole group of cirrhotic patients. This finding is supported by only one study that has investigated large intestinal permeability, showing an increased permeability in a mixed group of compensated and decompensated cirrhotic patients compared to healthy controls. ${ }^{4}$ 
Regarding the expression of TJ genes and proteins, we could only find mild and very limited alterations in duodenal and sigmoid biopsies of compensated cirrhotic patients compared to healthy controls (Chapter 3 ). These findings do not support the abovementioned functional permeability changes in vivo. Inconsistent results are also reported by others, showing altered and normal expression of TJ proteins in cirrhotic patients with barrier dysfunction. ${ }^{5,6}$ This indicates that the relation between functional and structural changes of the epithelial barrier in these patients needs further clarification. In addition to gene transcription, it has to be taken into account that posttranslational protein breakdown and/or modifications can contribute to "functional" protein levels. It should also be acknowledged that we evaluated paracellular permeability in the resting or basal state and not in response to stressors.

The observed increase in large intestinal permeability may be related to local inflammation due to intestinal oxidative stress. However, we could not find evidence for the occurrence of oxidative stress or low-grade inflammation in the mucosa of the large as well as the small intestine of patients with compensated cirrhosis (Chapter 4). These results are supported by the study of Assimakopoulos et al., ${ }^{7}$ showing similar duodenal levels of lipid hydroxyperoxides, markers of oxidative stress, in compensated cirrhotic patients and healthy controls. Though, increased levels were found in patients with decompensated cirrhosis when compared with those with compensated cirrhosis and healthy controls, ${ }^{7}$ suggesting that oxidative stress may be involved in the epithelial barrier dysfunction that has been reported in patients with complicated, decompensated cirrhosis. The question still to be answered is whether oxidative stress is a trigger or a result in this case.

Even though we only focused on paracellular permeability, barrier function includes more aspects and dimensions, such as the immune function and the intestinal microbiota, which can both be altered and thereby facilitate bacterial translocation. It has been suggested that alterations in barrier function, immune function and microbiota act in concert and synergistically promote bacterial translocation in cirrhosis. ${ }^{8}$ These factors will probably influence each other and may lead to a vicious circle in end stage complicated liver disease. ${ }^{8}$

Alterations in the intestinal microbiota can influence epithelial barrier function directly by affecting the expression of TJ proteins or indirectly via elevated levels of lipopolysaccharide (LPS)/endotoxin and the subsequent inflammatory response. ${ }^{9-13}$

In recent years, evidence of qualitative and quantitative changes in the fecal microbiota and their metabolic activity of cirrhotic patients is rapidly accumulating, ${ }^{14-24}$ while data on the microbiota biopsies are largely lacking. We characterized the fecal as well as the mucosa-associated microbiota in a heterogeneous group of (de)compensated cirrhotic patients and healthy controls by using IS-pro, a validated 16S-23S interspacer (IS) region-based profiling method (Chapter 5). Fecal and duodenal microbiota compositions were found to be different between patients with 
compensated cirrhosis and healthy controls. The observed differences in fecal microbiota were even more pronounced with progression of liver cirrhosis, i.e. in patients with decompensated cirrhosis and based on Child-Pugh classification, which is also found in one other recent study. ${ }^{16}$ Further studies are needed to show the potential of the observed microbial perturbations as disease progression marker using IS-profiling. This technique has the advantage that it is easy to implement in clinical practice and enables analysis of individual samples.

Furthermore, we found clear indications for involvement of the duodenal microbiota in cirrhosis and possible associations with small intestinal permeability. These findings provide new leads to further unravel the role of the small intestine in patients with cirrhosis, and warrants further study.

The observed microbial perturbations in patients with liver cirrhosis may be affected by host characteristics or etiological factors, such as alcohol use, ${ }^{14,25}$ dietary intake ${ }^{26}$ and obesity, ${ }^{27}$ but may also be associated with cirrhosis itself, for instance through changes in bile acid synthesis. ${ }^{28}$ Bile acids inhibit bacterial proliferation directly and indirectly via binding to the transcription factor farnesoid $X$ receptor (FxR). ${ }^{29}$ It has recently been suggested that the concentration of bile acids in the intestine may be decreased in cirrhosis, resulting in overgrowth of bacteria and in reduced numbers of bile acid $7 \alpha$-dehydroxylating bacteria (Clostridium cluster XIVa) in the colon. ${ }^{28}$ The latter assumption is in line with the higher $F / B$ ratio found in fecal samples of compensated cirrhotic patients when compared to healthy controls (Chapter 5).

At present, studies on the role of the intestinal microbiota shift from compositional to functional activity. In line with the potential role of bile acids and the reported saccharolytic fermentation (i.e. with the productions of beneficial short chain fatty acids), versus proteolytic fermentation (i.e. with the production of toxic phenolic compounds and amines), it would be relevant to extent future studies on the microbiota focusing on the metagenome and on key metabolic processes to further unravel its role in the pathophysiology of liver cirrhosis.

\section{The role of the intestine in chronic liver diseases}

Evidence for intestinal epithelial barrier dysfunction facilitating bacterial translocation as demonstrated by increased serum endotoxin levels, has been shown in animal models of several chronic liver diseases, such as alcoholic liver disease (ALD) and NAFLD. ${ }^{30,31}$ We conducted an animal study and compared two mouse models with diet-induced NAFLD, i.e. C57BI/6J mice with hepatic steatosis and low density lipoprotein knockout out (Ldlr-/-) mice with early steatohepatitis (Chapter 6). We found no indications for an increased permeability or an altered expression of TJ genes in small and large intestinal mucosa. We also observed no changes in the expression of oxidative stress related genes, nor in smooth muscle contractility. These results suggest that the intestine does not play an important role in the progression of 
steatosis to steatohepatitis in these mouse models. The findings may be due to the animal models used, in which we gave a high-fat and high-cholesterol diet to mimic early onset hepatic inflammation, but only for three weeks. Other animal studies have shown that especially the diet itself, e.g. high in fat or in fructose given for more than 8 weeks, may increase intestinal permeability. ${ }^{32-34}$ We were not able to evaluate the influence of diet only, since a control group on a normal diet was not included.

Overall, studies on intestinal permeability in animals and patients with NAFLD are limited and results are conflicting. Evidence for an increased permeability is more pronounced in animals and patients with ALD, confirming again the disruptive effects of alcohol on the epithelial barrier. These findings suggest that the underlying etiology is an important contributing factor to barrier dysfunction in animals and patients with chronic liver diseases.

\section{Non-invasive diagnostics}

Liver biopsy is an invasive procedure associated with morbidity, such as pain and bleeding with a mortality rate of 1 in 10,000 and high costs. ${ }^{35}$ The number of patients with chronic liver diseases is increasing, especially due to the progressive epidemics of obesity. Therefore, the demand for non-invasive techniques to assess the degree of fibrosis and inflammation for diagnosis and follow-up is growing. Transient elastography, i.e. FibroScan, is the most widely used and best validated imaging technique, with a high diagnostic yield in cirrhosis. ${ }^{36}$ However, the technique is limited applicable in obesity and patients with ascites, is operator depended and local availability differs. ${ }^{36}$ Combinations of serum biomarker panels have also proven to accurately detect cirrhosis in patients with chronic liver diseases. ${ }^{36}$ We confirmed the efficacy of serological testing with a combination of 5 routine parameters, i.e. ALT, GGT, bilirubin, albumin and thrombocytes (Chapter 8 ), showing a sensitivity of $71 \%$ and a specificity of $84 \%$. These parameters mainly reflect alterations in hepatic function, i.e. disease severity. Besides gaining insight into the degree of fibrosis, it is also important to follow hepatic function over time and to evaluate the functional metabolic capacity of the liver. Breath tests using ${ }^{13} \mathrm{C}$-labeled probe drugs, for example, can be used to assess the functional metabolic capacity of the liver in patients with chronic liver diseases, but are easily affected by many factors such as hepatic perfusion and extraction ratio of the probe drug that is used, genetic metabolic polymorphisms and concomitant drug use (Chapter 7).

Furthermore, we have shown the potency of a specific exhaled VOC profile as a noninvasive biomarker to predict the presence of compensated cirrhosis among a heterogeneous group of patients with chronic liver diseases. This profile of 11 VOCs was found to have a higher accuracy than a panel of routine serological markers, showing a sensitivity of $83 \%$ and a specificity of $87 \%$. The negative predictive value of $84 \%$ indicates that VOCs may aid in reducing the number of liver biopsies in clinical practice. However, these promising findings need to be further validated and 
confirmed in large cohorts including well-diagnosed and phenotyped patients, based on liver biopsy. An advantage of exhaled VOCs is that they may reflect metabolic liver function and that the identified compounds may provide leads for pathophysiological processes.

In addition, exhaled air is very easy and safe to obtain, can be performed repeatedly and hence may indicate disease progression. Recent advances in analytical techniques and development of easy-to-use, cheap, hand-held equipment, e.g. the electronic nose, which can sense and recognize odors and flavors, may enable (real-time) detection and analysis of VOCs in routine clinical practice. This may also enable studies with follow-up of large cohorts of patients over time to get further insight in risk factors for the increased disease progression, such as the development of steatohepatitis (NASH) among subjects with hepatic steatosis or the development of complications among patients with liver cirrhosis.

\section{Future perspectives}

Up to now, evidence for barrier dysfunction of the small intestine in patients with compensated liver cirrhosis is limited. The barrier seems however to be impaired in the large intestine. Therefore, future studies should be conducted to further address the involvement of the large intestine regarding permeability, both functionally and structurally, in bacterial translocation and in progression towards decompensated cirrhosis.

Barrier function may differ depending on the underlying etiology as changes in intestinal permeability are more pronounced in liver diseases related to alcohol and therefore further studies on barrier function in cirrhosis should be performed in subgroups of patients with specific etiologies.

It is tempting to assume that the barrier dysfunction of the large intestine is related to the observed alterations in the fecal microbiota composition. Better understanding of these alterations, especially with regard to the functional activity using state-of-the-art molecular techniques, could further elucidate the interaction between the microbiota, luminal contents, e.g. bile acids, and intestinal barrier, thereby contributing to a better understanding of the pathophysiology and disease progression of cirrhosis.

Further insight into the pathophysiology by paying attention to early and non-invasive diagnosis is also important and analysis of exhaled VOCs is promising.

The intestine is an intriguing organ and this thesis shows that its function and structure, i.e. epithelial barrier and microbiota composition, are involved in compensated liver cirrhosis. It would be relevant to further investigate whether the observed cirrhosis-related alterations in the intestine can be restored, for example by influencing microbiota composition, and thereby prevent progression towards end stage, complicated, liver disease. 


\section{References}

1. Groschwitz KR, Hogan SP. Intestinal barrier function: molecular regulation and disease pathogenesis. J Allergy Clin Immunol 2009;124:3-20; quiz 1-2.

2. Pijls KE, Jonkers DM, Elamin EE, Masclee AA, Koek GH. Intestinal epithelial barrier function in liver cirrhosis: an extensive review of the literature. Liver international : official journal of the International Association for the Study of the Liver 2013;33:1457-69.

3. Elamin E, Masclee A, Dekker J, Jonkers D. Ethanol metabolism and its effects on the intestinal epithelial barrier. Nutr Rev 2013; [In press].

4. Norman K, Pirlich M, Schulzke JD, et al. Increased intestinal permeability in malnourished patients with liver cirrhosis. Eur J Clin Nutr 2012.

5. Assimakopoulos SF, Tsamandas AC, Tsiaoussis GI, et al. Altered intestinal tight junctions' expression in patients with liver cirrhosis: a pathogenetic mechanism of intestinal hyperpermeability. Eur J Clin Invest.

6. Du Plessis J, Vanheel H, Janssen CE, et al. Activated intestinal macrophages in patients with cirrhosis release NO and IL-6 that may disrupt intestinal barrier function. J Hepatol 2013.

7. Assimakopoulos SF, Tsamandas AC, Tsiaoussis GI, et al. Intestinal mucosal proliferation, apoptosis and oxidative stress in patients with liver cirrhosis. Ann Hepatol 2013;12:301-7.

8. Wiest R, Lawson M, Geuking M. Pathological bacterial translocation in liver cirrhosis. J Hepatol 2013.

9. Qin H, Zhang Z, Hang X, Jiang Y. L. plantarum prevents enteroinvasive Escherichia coli-induced tight junction proteins changes in intestinal epithelial cells. BMC Microbiol 2009;9:63.

10. Karczewski J, Troost FJ, Konings I, et al. Regulation of human epithelial tight junction proteins by Lactobacillus plantarum in vivo and protective effects on the epithelial barrier. Am J Physiol Gastrointest Liver Physiol;298:G851-9.

11. Forsythe RM, Xu DZ, Lu Q, Deitch EA. Lipopolysaccharide-induced enterocyte-derived nitric oxide induces intestinal monolayer permeability in an autocrine fashion. Shock 2002;17:180-4.

12. Bruewer M, Luegering A, Kucharzik T, et al. Proinflammatory cytokines disrupt epithelial barrier function by apoptosis-independent mechanisms. J Immunol 2003;171:6164-72.

13. Bruewer $M$, Utech $M$, Ivanov Al, Hopkins AM, Parkos CA, Nusrat A. Interferon-gamma induces internalization of epithelial tight junction proteins via a macropinocytosis-like process. FASEB J 2005;19:923-33.

14. Chen Y, Yang F, Lu H, et al. Characterization of fecal microbial communities in patients with liver cirrhosis. Hepatology 2011;54:562-72.

15. Liu CL, Ai HW, Wang WP, et al. Comparison of $16 \mathrm{~S}$ rRNA gene PCR and blood culture for diagnosis of neonatal sepsis. Arch Pediatr 2014;21:162-9.

16. Bajaj JS, Heuman DM, Hylemon PB, et al. Altered profile of human gut microbiome is associated with cirrhosis and its complications. J Hepatol 2014;60:940-7.

17. Bajaj JS, Hylemon PB, Ridlon JM, et al. Colonic mucosal microbiome differs from stool microbiome in cirrhosis and hepatic encephalopathy and is linked to cognition and inflammation. Am J Physiol Gastrointest Liver Physiol 2012;303:G675-85.

18. Bajaj JS, Ridlon JM, Hylemon PB, et al. Linkage of gut microbiome with cognition in hepatic encephalopathy. Am J Physiol Gastrointest Liver Physiol 2012;302:G168-75.

19. Chen Y, Qin N, Guo J, et al. Functional gene arrays-based analysis of fecal microbiomes in patients with liver cirrhosis. BMC Genomics 2014;15:753.

20. Qin N, Yang F, Li A, et al. Alterations of the human gut microbiome in liver cirrhosis. Nature 2014;513:59-64.

21. Wei X, Yan X, Zou D, et al. Abnormal fecal microbiota community and functions in patients with hepatitis B liver cirrhosis as revealed by a metagenomic approach. BMC Gastroenterol 2013;13:175.

22. Zhang Z, Zhai H, Geng J, et al. Large-scale survey of gut microbiota associated with MHE Via 16S rRNAbased pyrosequencing. Am J Gastroenterol 2013;108:1601-11.

23. Lu H, Wu Z, Xu W, Yang J, Chen Y, Li L. Intestinal microbiota was assessed in cirrhotic patients with hepatitis B virus infection. Intestinal microbiota of HBV cirrhotic patients. Microb Ecol 2011;61:693703. 
24. Kakiyama G, Hylemon PB, Zhou H, et al. Colonic inflammation and secondary bile acids in alcoholic cirrhosis. Am J Physiol Gastrointest Liver Physiol 2014;306:G929-37.

25. Vassallo G, Mirijello A, Ferrulli A, et al. Review article: Alcohol and gut microbiota - the possible role of gut microbiota modulation in the treatment of alcoholic liver disease. Aliment Pharmacol Ther 2015;41:917-27.

26. Xu Z, Knight R. Dietary effects on human gut microbiome diversity. Br J Nutr 2015;113 Suppl:S1-5.

27. Turnbaugh PJ, Hamady M, Yatsunenko T, et al. A core gut microbiome in obese and lean twins. Nature 2009;457:480-4.

28. Ridlon JM, Alves JM, Hylemon PB, Bajaj JS. Cirrhosis, bile acids and gut microbiota: unraveling a complex relationship. Gut microbes 2013;4:382-7.

29. Inagaki T, Moschetta A, Lee YK, et al. Regulation of antibacterial defense in the small intestine by the nuclear bile acid receptor. Proc Natl Acad Sci U S A 2006;103:3920-5.

30. Keshavarzian A, Farhadi A, Forsyth $C B$, et al. Evidence that chronic alcohol exposure promotes intestinal oxidative stress, intestinal hyperpermeability and endotoxemia prior to development of alcoholic steatohepatitis in rats. J Hepatol 2009;50:538-47.

31. Brun P, Castagliuolo I, Di Leo V, et al. Increased intestinal permeability in obese mice: new evidence in the pathogenesis of nonalcoholic steatohepatitis. Am J Physiol Gastrointest Liver Physiol 2007;292:G518-25.

32. Suzuki T, Hara H. Dietary fat and bile juice, but not obesity, are responsible for the increase in small intestinal permeability induced through the suppression of tight junction protein expression in LETO and OLETF rats. Nutr Metab (Lond) 2010;7:19.

33. Moreira AP, Texeira TF, Ferreira AB, Peluzio Mdo C, Alfenas Rde C. Influence of a high-fat diet on gut microbiota, intestinal permeability and metabolic endotoxaemia. The British journal of nutrition 2012;108:801-9.

34. Sellmann C, Priebs J, Landmann M, et al. Diets rich in fructose, fat or fructose and fat alter intestinal barrier function and lead to the development of nonalcoholic fatty liver disease over time. The Journal of nutritional biochemistry 2015;26:1183-92.

35. Rockey DC, Caldwell SH, Goodman ZD, Nelson RC, Smith AD. Liver biopsy. Hepatology 2009;49:101744.

36. Patel K, Bedossa P, Castera L. Diagnosis of liver fibrosis: present and future. Semin Liver Dis 2015;35:166-83. 
154 
Summary 


\section{Summary}

This thesis focused on two aspects of liver cirrhosis: the role of the intestine in the pathophysiology and non-invasive diagnostics. Previous studies indicate that the intestine is an important co-factor in the pathophysiology of chronic liver diseases, cirrhosis and cirrhosis-related complications, e.g. by means of bacterial translocation. In this thesis, we aimed to investigate the role of the intestine in patients with compensated cirrhosis, i.e. without complications, paying attention to both the small and large intestine, with specific emphasis on epithelial barrier function.

In the search for non-invasive tests, we addressed the role of breath analyses for diagnosis of chronic liver diseases, especially cirrhosis at an early stage, as this is important to prevent progression towards end stage, complicated, liver disease.

\section{The role of the intestine}

Chapter 2 provides an overview of the current evidence of intestinal epithelial barrier dysfunction in human chronic liver diseases and cirrhosis. Dysfunction of the intestinal epithelial barrier has been found in patients with chronic liver diseases, but the evidence is more convincing in patients with cirrhosis, especially in those with complications. However, these studies have mainly investigated small and whole intestinal permeability by measuring the excretion of orally administered test markers in mixed groups of patients with compensated and/or decompensated cirrhosis. Information on the large intestine and compensated cirrhotic patients as well as on tight junction (TJ) structure is scarce. The barrier dysfunction can result from the effects of etiological factors for cirrhosis, such as alcohol consumption or high fat intake (obesity). On the other hand, cirrhosis itself is suggested to lead to barrier dysfunction via the effects of portal hypertension, the probably altered intestinal microbiota, inflammation and oxidative stress, each of which have been discussed in this chapter.

In Chapter 3, paracellular permeability of both the small and large intestine is investigated in patients with compensated cirrhosis and compared to age, gender and BMI-matched healthy controls, as increased permeability might be a risk factor for the development of complications. Intestinal permeability was assessed by a validated multi-sugar test as well as by the expression of key TJ genes and proteins in duodenal and sigmoid biopsies. Interestingly, we found an increased permeability of the large intestine in compensated cirrhotic patients, which warrants further investigation. Gastroduodenal and small intestinal permeability were not altered. However, in a subgroup of alcohol-related cirrhotic patients, small intestinal permeability was found to be increased, confirming the pronounced and disruptive effects of alcohol and its oxidative metabolites on the epithelial barrier. 
On the other hand, we found only mild and very limited alterations regarding the expression of TJ genes and proteins in duodenal and sigmoid biopsies of cirrhotic patients. These findings did not support the observed functional permeability changes in vivo, suggesting that the relation between functional and structural changes of the epithelial barrier needs further clarification.

In the following chapter, we were interested to find evidence whether intestinal oxidative stress occurs in cirrhosis. Therefore, in the study described in Chapter 4, parameters of oxidative stress were measured in both duodenal and sigmoid mucosa of compensated cirrhotic patients and healthy controls. Only gene transcription of glutathione synthetase and reductase were found to be different between both groups. No alterations were observed for other genes related to oxidative stress, nor for glutathione, glutathione disulphide, glutathione/disulphide ratio or intestinal myeloperoxidase and fecal calprotectin concentrations. These findings indicate that intestinal oxidative stress is not a major mechanism contributing to the epithelial barrier dysfunction observed in patients with compensated cirrhosis.

Alterations in the intestinal microbiota can also affect the epithelial barrier, both directly and indirectly. Recent studies have mainly investigated the fecal microbiota of cirrhotic patients, while information on the microbiota of the intestinal mucosa is very limited. In Chapter 5, the fecal and mucosa-associated microbiota is investigated in a heterogeneous group of patients with (de)compensated cirrhosis and compared with healthy controls using a validated 16S-23S interspacer (IS) region-based profiling method. The results showed differences in fecal and duodenal microbiota composition between compensated cirrhotic patients and healthy controls, as shown by an increased fecal and duodenal Firmicutes to Bacteroidetes (F/B) ratio in these patients, and alterations in richness and diversity of the phyla Firmicutes and Bacteroidetes. Furthermore, an altered microbiota composition was also found in duodenal biopsies, as well as possible associations with the lactulose/rhamnose ratio indicating small intestinal permeability. No alterations were observed in the microbiota composition of the sigmoid. The observed differences in fecal microbiota were more pronounced with progression of liver cirrhosis, i.e. in patients with decompensated cirrhosis. Furthermore, patients with (de)compensated cirrhosis were found to cluster separately from healthy controls and showed distinct clusters based on the Child-Pugh classification, showing potential of the intestinal microbiota as marker to monitor disease progression.

Overall, the above findings, using IS-profiling which is easy to implement in clinical practice, confirm the recently reported presence of microbial perturbations in compensated cirrhotic patients. This may contribute to the observed increased permeability, but may also affect host function, e.g. via its metabolic activity and/or effects on the immune system. 
To further unravel the pathophysiology of the intestinal involvement in chronic liver diseases, in particular of non-alcoholic fatty liver disease (NAFLD), we conducted an animal study and compared two mouse models with diet-induced NAFLD. The results, described in Chapter 6, confirmed the presence of hepatic steatosis in wild type C57BI/6J (WT) mice and early steatohepatitis in low density lipoprotein knock-out (Ldlr-/-) mice after a 3-week period of high-fat and high-cholesterol feeding. However, no major differences were found regarding epithelial barrier function and oxidative stress in ileum and sigmoid mucosa between both mouse models. Duodenal smooth muscle contractility was also not found to be different. These results suggest that the intestine does not play a major role in the onset of hepatic inflammation in these NAFLD mouse models investigated.

\section{Non-invasive diagnostics}

In Chapters 7 and 8, we addressed the role of breath analyses as non-invasive alternatives to liver biopsy for diagnosis of chronic liver diseases, especially cirrhosis at an early stage.

In Chapter 7, we appraised the value of breath tests using ${ }^{13} \mathrm{C}$-labeled probe drugs for the evaluation of metabolic liver function and discussed the role of cytochrome P450 enzymes in the metabolism of the different probe drugs, with particular emphasis on aminopyrine. Although, the perfect probe drug is not yet available, ${ }^{13} \mathrm{C}_{2}$-aminopyrine and ${ }^{13} \mathrm{C}$-methacetin can play a role in evaluating the capacity of the microsomal liver function and may be useful in the follow-up of patients with chronic liver diseases. Furthermore, CYP2C19 is an important enzyme in the $\mathrm{N}$-demethylation of aminopyrine and polymorphisms in this gene can influence breath test values. Together with other potential confounding factors, such as concomitant drug use, this should be kept in mind when interpreting the ${ }^{13} \mathrm{C}_{2}$-aminopyrine breath test in clinical practice.

In addition to breath tests using ${ }^{13} \mathrm{C}$-labeled probe drugs, analysis of volatile organic compounds (VOCs) in exhaled air has been suggested useful for the diagnosis of several diseases, including intestinal and liver diseases. In Chapter 8, we aimed to investigate whether VOCs can predict the presence of cirrhosis within a heterogeneous group of chronic liver disease patients and to compare this with a panel of routine serological markers. We demonstrated that a combination of routine serological markers, i.e. GGT, ALT, bilirubin, albumin, and thrombocytes, can discriminate cirrhotic patients from patients with chronic liver diseases with a sensitivity of $71 \%$ and a specificity of $84 \%$. However, an exhaled profile of 11 VOCs was found to predict the presence of compensated cirrhosis with a sensitivity of $83 \%$, and a specificity of $87 \%$. The combination of both did not further improve the diagnostic accuracy. Exhaled VOCs appear to be a promising non-invasive biomarker for cirrhosis that may reflect 
metabolic liver function and may help to reduce liver biopsies. Nevertheless, these findings should be further validated in larger cohorts with well-diagnosed patients.

In Chapter 9, we discussed the major findings of this thesis and proposed future research topics. Overall, this thesis emphasis that the intestine, i.e. epithelial barrier and microbiota composition, is involved in compensated liver cirrhosis, and thereby may be a risk factor for development of complications. In addition, exhaled VOCs appear to be a promising non-invasive alternative to liver biopsy for diagnosing cirrhosis at an early stage. 
Samenvatting 


\section{Samenvatting}

In dit proefschrift hebben we gekeken naar de rol van de darm in de pathofysiologie van levercirrose en naar non-invasieve markers om levercirrose te diagnosticeren. Op basis van voorgaande studies zijn er aanwijzingen dat de darm via bacteriële translocatie een belangrijke cofactor is in de pathofysiologie van chronische leverziekten, cirrose en complicaties van cirrose. In dit proefschrift hebben we de rol van de darm in patiënten met levercirrose zonder complicaties onderzocht. Daarbij hebben we aandacht besteed aan zowel de dunne als de dikke darm en de nadruk gelegd op de epitheliale barrièrefunctie.

In de zoektocht naar niet-invasieve testen hebben we gekeken naar de rol van adem analyses in de diagnose van chronische leverziekten en cirrose (zonder complicaties). Dit is belangrijk om progressie van chronische leverziekten met de ontwikkeling van complicaties te voorkomen.

\section{De rol van de darm}

Hoofdstuk 2 geeft een overzicht van het huidige bewijs voor een verstoring van de epitheliale barrière van de darm in chronische leverziekten en cirrose. Diverse studies tonen een verstoring van de intestinale permeabiliteit in patiënten met chronische leverziekten die het meest duidelijk is in patiënten met cirrose en vooral in patiënten met complicaties. Deze studies hebben echter voornamelijk de permeabiliteit van de dunne en de gehele darm onderzocht door de excretie van oraal toegediende test markers te meten in gemengde groepen patiënten met gecompenseerde en/of gedecompenseerde cirrose. Er zijn echter nauwelijks gegevens over de dikke darm, over patiënten met gecompenseerde levercirrose en over de structuur en expressie van de 'tight junction' eiwitten. Verstoring van de barrière kan het resultaat zijn van de effecten van etiologische factoren van cirrose zoals alcohol of een hoge consumptie van vetten (obesitas). Echter, cirrose kan ook zelf leiden tot een verstoring van de barrière via de effecten van portale hypertensie, de veranderde intestinale microbiota, aanwezigheid van ontsteking en oxidatieve stress.

Aangezien een verhoogde darm permeabiliteit een risicofactor zou kunnen zijn voor de ontwikkeling van complicaties in patiënten met levercirrose, is in hoofdstuk 3 de paracelullaire permeabiliteit van zowel de dunne als de dikke darm in patiënten met gecompenseerde levercirrose onderzocht en vergeleken met gezonde controles die waren gematcht voor leeftijd, geslacht en BMI. De intestinale permeabiliteit werd bepaald met behulp van een gevalideerde 'multi-sugar' test en middels het analyseren van de expressie van belangrijke 'tight junction' genen en eiwitten in duodenum en sigmoid biopten. We vonden een verhoogde permeabiliteit van de dikke darm in gecompenseerde levercirrose patiënten. Deze nieuwe bevinding behoeft verder 
onderzoek. De gastroduodenale en dunne darm permeabiliteit waren niet verschillend ten opzichte van de controlepersonen. De dunne darm permeabiliteit was echter wel verhoogd in een subgroep van patiënten met alcohol gerelateerde levercirrose en dit bevestigt de schadelijke effecten van alcohol en zijn oxidatieve metabolieten op de epitheliale barrière.

Met betrekking tot de expressie van tight junction genen en eiwitten in duodenum en sigmoid biopten van cirrose patiënten, werden slechts beperkte en kleine veranderingen gevonden. Deze waren niet geheel in lijn met de in vivo geobserveerde functionele veranderingen in de permeabiliteit en dit suggereert dat er behoefte is aan verder onderzoek naar de relatie tussen functionele en structurele veranderingen in de epitheliale barrière.

In het volgende hoofdstuk waren we geïnteresseerd of er aanwijzingen zijn voor het bestaan van intestinale oxidatieve stress in cirrose. Zodoende zijn in hoofdstuk 4 parameters van oxidatieve stress gemeten in duodenum en sigmoid mucosa van patiënten met gecompenseerde levercirrose en gezonde controles. Alleen de gentranscriptie van glutathion synthetase en reductase waren verschillend tussen beide groepen. Er werden geen veranderingen gevonden in andere genen gerelateerd aan oxidatieve stress, en ook niet in glutathion, glutathion disulphide, glutathion/ disulphide ratio, of intestinale myeloperoxidase en calprotectine concentraties. Deze bevindingen wijzen erop dat intestinale oxidatieve stress geen belangrijk mechanisme lijkt te zijn voor de verstoorde darmbarrière die we zagen bij patiënten met gecompenseerde levercirrose.

Behalve ontsteking en oxidatieve stress, zouden veranderingen in de intestinale microbiota ook van invloed kunnen zijn op de epitheliale barrièrefunctie, zowel door directe als indirecte effecten (via bacteriële metabolieten). Recente studies hebben voornamelijk de fecale microbiota onderzocht in patiënten met levercirrose. Er is tot op heden heel weinig informatie over de microbiota in de intestinale mucosa. In hoofdstuk 5 is daarom de fecale en de mucosa-geassocieerde microbiota onderzocht in een heterogene groep van patiënten met gecompenseerde en gedecompenseerde cirrose en vergeleken met gezonde controles. Hierbij werd gebruik gemaakt van de gevalideerde '16S-23S interspacer (IS) region-based profiling' methode. De resultaten toonden verschillen in de compositie van de fecale en duodenale microbiota tussen gecompenseerde cirrose patiënten en gezonde controles: een verhoogde verhouding van Firmicutes ten opzichte van Bacteroidetes, en veranderingen in 'richness' en diversiteit van de phyla Firmicutes en Bacteroidetes werden gevonden. Daarnaast vonden we ook een verhoogde verhouding van Firmicutes ten opzichte van Bacteroidetes en een verhoogde 'richness' van de phyla Firmicutes in duodenum biopten van gecompenseerde cirrose patiënten in vergelijking met gezonde controles, en mogelijke associaties met de lactulose/rhamnose ratio als indicator voor dunne darm permeabiliteit. $\mathrm{Er}$ werden geen veranderingen getoond in de microbiota 
compositie van het sigmoid. De geobserveerde verschillen in de fecale microbiota waren meer uitgesproken naarmate de ziekte vorderde, dat wil zeggen in patiënten met gedecompenseerde cirrose. Verder vonden we dat patiënten met ge(de)compenseerde cirrose apart clusterden van gezonde controles en verschillende clusters toonden gebaseerd op de Child-Pugh classificatie voor de ziekte-ernst. Deze resultaten tonen de potentie van de intestinale microbiota als marker om progressie van ziekte te monitoren.

De bovenstaande bevindingen die gedaan zijn met behulp van IS-profiling, hetgeen makkelijk implementeerbaar is in de klinische praktijk, bevestigen de recent gerapporteerde aanwezigheid van microbiële verstoringen in patiënten met gecompenseerde levercirrose. Deze verstoringen zouden kunnen bijdragen aan de geobserveerde verhoogde permeabiliteit, maar zouden ook effect kunnen hebben op de gastheer via bijvoorbeeld de metabole activiteit en/of de effecten op het immuun systeem.

Om de betrokkenheid van de darm verder uit te zoeken in chronische leverziekten en in het bijzonder in niet-alcoholische vette leverziekte (NAFLD), hebben we een dierstudie opgezet en twee muismodellen met voeding-geïnduceerde NAFLD vergeleken. De resultaten, die beschreven zijn in hoofdstuk 6, bevestigden de aanwezigheid van lever steatose in "wild type C57BI/6J"(WT) muizen en vroege steatohepatitis in de "low density lipoprotein knock-out" (Ldlr-/-) muizen na een vet en cholesterolrijke voeding van drie weken. Er werden echter geen grote verschillen gevonden tussen beide muismodellen voor wat betreft de epitheliale barrière functie en oxidatieve stress in ileum en sigmoid mucosa. Er was ook geen verschil in de contractiliteit van glad spierweefsel van het duodenum. Deze resultaten wijzen erop dat de darm geen grote rol speelt in het ontstaan van lever-inflammatie in de onderzochte NAFLD muismodellen.

\section{Niet-invasieve diagnostiek}

In hoofdstuk 7 en 8 hebben we gekeken naar de rol van adem analyses als nietinvasieve alternatieven van een leverbiopsie voor de diagnose van chronische leverziekten en voornamelijk van cirrose in een vroeg stadium (zonder complicaties).

In hoofdstuk 7 hebben we een overzicht gegeven van de waarde van ademtesten die gebruik maken van ${ }^{13} \mathrm{C}$-gelabelde test drugs voor de evaluatie van de metabole leverfunctie. Daarnaast hebben we de rol van cytochroom P450 enzymen in het metabolisme van verschillende test drugs bediscussieerd en speciale aandacht geschonken aan aminopyrine. Ondanks dat de perfecte test drug nog niet beschikbaar is, kunnen ${ }^{13} \mathrm{C}$-aminopyrine en ${ }^{13} \mathrm{C}$-methacetine een rol spelen in de evaluatie van de capaciteit van de microsomale leverfunctie en bruikbaar zijn in het monitoren van patiënten met chronische leverziekten. Daarnaast is CYP2C19 een belangrijk enzym in 
de $\mathrm{N}$-demethylatie van aminopyrine en polymorfismen in dit gen kunnen de uitslag van ademtesten beïnvloeden. Hier dient samen met andere mogelijke variabelen zoals gelijktijdig gebruik van geneesmiddelen rekening mee te worden gehouden bij de interpretatie van de ${ }^{13} \mathrm{C}$-aminopyrine ademtest in de klinische praktijk.

Naast deze ademtesten die gebruik maken van ${ }^{13} \mathrm{C}$-gelabelde test drugs, zijn er aanwijzingen dat de analyse van zogenaamde volatile organic compounds (VOCs) in uitademingslucht bruikbaar is voor de diagnose van verschillende ziektes waaronder darm- en leverziekten. In hoofdstuk 8 hebben we daarom getracht te onderzoeken of VOCs in de uitgeademde lucht, de aanwezigheid van cirrose binnen een heterogene groep van patiënten met chronische leverziekten kunnen voorspellen. Daarnaast is dit vergeleken met een panel van serologische markers die in de dagelijkse praktijk worden gebruikt. We hebben aangetoond dat een combinatie van serologische markers bestaande uit $\gamma-\mathrm{GT}$, ALAT, bilirubine, albumine en trombocyten, patiënten met cirrose kan onderscheiden van patiënten met chronische leverziekten met een sensitiviteit van $71 \%$ en een specificiteit van $84 \%$. Een profiel van 11 VOCs in de uitademingslucht voorspelde de aanwezigheid van gecompenseerde levercirrose echter met een sensitiviteit van $83 \%$ en een specificiteit van $87 \%$. De diagnostische nauwkeurigheid verbeterde niet door beide benaderingen te combineren. Uitgeademde VOCs lijken een veelbelovend niet-invasieve marker voor cirrose, die de metabole lever functie kan reflecteren en het aantal leverbiopsies kan doen verminderen. Deze bevindingen moeten echter wel nog gevalideerd worden in grotere cohorten met goed gediagnosticeerde patiënten.

In hoofdstuk 9 zijn de voornaamste bevindingen van dit proefschrift bediscussieerd en zijn voorstellen gedaan voor toekomstige onderzoeken. Over het algemeen geeft dit proefschrift duidelijke aanwijzingen dat de darm, en dan met name de epitheliale barrière en de microbiota, veranderd zijn in gecompenseerde levercirrose, en zodoende een risicofactor kunnen zijn voor de ontwikkeling van complicaties. Daarnaast lijken uitgeademde VOCs een veelbelovend niet-invasief alternatief te zijn voor leverbiopsie om cirrose in een vroeg stadium te kunnen diagnosticeren. 
Valorisation addendum 


\section{Valorisation addendum}

Liver cirrhosis is the end result of a variety of chronic liver diseases, predominantly related to alcohol consumption, viral infections and metabolic disorders. It is characterized by distortion of normal liver architecture encompassing diffuse parenchymal fibrosis, nodules and vascular remodeling that lead to impaired liver function. ${ }^{1}$ The liver is a central organ and is very important for whole body homeostasis. According to the National Center for Health Statistics, cirrhosis and chronic liver diseases are the twelfth leading cause of death in the United States. ${ }^{2}$ In Europe, $1.8 \%$ of all deaths are caused by cirrhosis, accounting for approximately 170.000 deaths per year. ${ }^{3}$ Worldwide, the number of patients progressing to liver cirrhosis is expected to rise, especially due to the obesity epidemic associated with the metabolic syndrome and non-alcoholic fatty liver disease (NAFLD), but also because of aging of patients with chronic viral liver diseases.

Liver cirrhosis is an asymptomatic disorder until complications, i.e. decompensation, because of portal hypertension occur. These complications, including variceal hemorrhage, spontaneous bacterial peritonitis (SBP) and hepatic encephalopathy are mainly responsible for the high morbidity and mortality, accounting for frequent hospital admissions and for 1.03 million deaths per year worldwide, 170.000 per year in Europe and 33.539 per year in the United States. Compared to patients with compensated cirrhosis, patients with decompensated cirrhosis report a poorer quality of life, which inevitably also leads to increased health-care utilization.

Despite recent advances in cause-specific treatments, liver transplantation is still the only effective and expensive curative option in cirrhotic patients with complications or hepatocellular carcinoma. Annually, 5500 liver transplants are performed in Europe with cirrhosis being the main indication.

As a result, liver cirrhosis is responsible for considerable health-care utilization and represents a large economic burden with high direct health-care costs as well as indirect costs related to work absenteeism and reduced work productivity. In 2004 in the United States, annual direct costs for chronic liver diseases and cirrhosis, excluding chronic hepatitis $\mathrm{C}$, were estimated to be $\$ 2.5$ billion with even higher estimates for indirect costs.

Considering these figures and the increasing prevalence of cirrhosis, further research to decrease the disease burden and health care costs of cirrhotic patients, is needed for patients as well as society. The results of this thesis will contribute in further insight into the pathophysiology of liver cirrhosis, thereby aiming to identify leads for preventing progression to end stage complicated liver disease. Furthermore, we aimed to identify non-invasive tools for diagnosing cirrhosis at an early stage and thereby prevent development of severe complications, e.g. by endoscopic screening of or 
prophylactic treatment for gastrointestinal varices, ultimately aiming to increase health-related quality of life.

Liver biopsy is still the current standard for a definite diagnosis, but is difficult in the follow-up as repetitive biopsies are not accepted by patients and many health care workers. It is an invasive procedure with significant complications, such as pain and bleeding with a mortality rate of 1 in 10.000 and high costs. ${ }^{4}$ Diagnostic tools to reduce the number of biopsies are therefore highly warranted.

Studies to find non-invasive alternatives for staging fibrosis are ongoing. As dynamic changes are currently considered to be involved in fibrogenesis, it is important to assess the functional metabolic capacity of the liver and search for markers that can measure ongoing pathophysiological processes and metabolic functions. Analysis of exhaled volatile organic compounds (VOCs) is already found to discriminate between cirrhosis and healthy controls, and was further studied in this thesis. VOCs have the advantage to reflect liver metabolic function and, therefore, may provide leads towards pathophysiological pathways. Furthermore, exhaled air collection is noninvasive, easy and requires only a minimal time investment. We found that VOCs can predict the presence of compensated cirrhosis among a heterogeneous group of patients with chronic liver diseases, with a higher accuracy than a panel of routine serological parameters. Therefore, VOCs may aid in reducing the number of liver biopsies in clinical practice, and will provide more insight into the pathophysiological processes and risk factors for disease progression. Furthermore, after validation of the current findings in other groups of patients, it would be of interest to further develop simple devices (i.e. sensors) to measure the VOCs, which can be implemented in daily clinical practice to monitor these patients over time. Thereby, these patients will not have to undergo (repetitive) invasive biopsies for diagnosing cirrhosis at an early stage to prevent development of complications, and results in an improved health-related quality of life.

Although chronic liver diseases, such as alcoholic liver disease and chronic infections by Hepatitis B and C, can lead to liver damage, fibrosis and ultimately cirrhosis, further insight is needed in (co-)factors increasing the risk of disease progression, i.e. to decompensated cirrhosis, and thereby aiming to decrease cirrhosis-related comorbidity and health care costs. In addition to the above-mentioned etiological factors, also the intestine may play a role, with its' barrier function aiming to prevent permeation of substances from the external (i.e. intestinal lumen) to the internal environment. However, studies are limited and often focused on the small intestine and/or one potential pathophysiological mechanism. This thesis has therefore focused on the role of the small and large intestine in patients with compensated cirrhosis. In particular, we demonstrated an impaired epithelial barrier function of the large intestine to be present in these patients. As the large intestine is colonized by a high number of (commensal) bacteria, the observed barrier dysfunction may enhance 
bacterial translocation and contribute to further liver damage and the development of cirrhosis-related complications, such as SBP. Reinforcement of the intestinal epithelial barrier may therefore have therapeutic potential. For example, larazotide acetate, a novel tight junction (TJ) regulator peptide that prevents TJ opening, showed promising results in patients with celiac disease. ${ }^{5}$ Furthermore, probiotics have shown to improve the epithelial integrity and reduce bacterial translocation. Results of a recent study showed that treatment with a probiotic reduced the risk of hospitalization and improved liver function and health-related quality of life in cirrhotic patients. ${ }^{6}$ We therefore suggest that therapeutic modalities to restore epithelial barrier function, especially in cirrhosis, should be further investigated.

In this thesis, we also demonstrated an altered composition of the fecal microbiota in patients with compensated cirrhosis, which was even more pronounced with progression of liver cirrhosis. Based on these findings, the role of the fecal microbiota as marker for disease progression needs further study. Furthermore, it would be of interest to correlate this to findings on the VOCs in exhaled air, as a large part of them may result from the intestinal microbiota. Remarkably, we also found clear indications for involvement of the duodenal microbiota in cirrhosis and possible associations with small intestinal permeability. Bacteria have been shown to influence intestinal epithelial barrier directly by affecting the expression of TJ proteins or indirectly via elevated levels of lipopolysaccharide (LPS)/endotoxin and the subsequent inflammatory response.

Evidence indicates that the intestinal microbiota is an important player in the pathophysiology of several chronic liver diseases and development of cirrhosis-related complications. Modulating the intestinal microbiota by, for example, antibiotics or pre/probiotics may therefore influence the disease course and outcome of liver cirrhosis. In fact, these agents have proven successful in the prevention and treatment of several cirrhosis-related complications. Recently, interest is also growing in fecal microbiota transplantation for the treatment of several diseases associated with dysbiosis; the results look promising and may be an alternative treatment for patients with hepatic encephalopathy and SBP.

We think that better understanding of the alterations in the intestinal microbiota, especially with regard to their functional activity using state-of-the-art molecular techniques, will further elucidate the interaction between the microbiota and the intestine, leading to new insights in the pathogenesis, disease progression and may improve therapeutic strategies for cirrhosis. Future research will confirm the evidence for targeting the intestinal microbiota as a therapeutic strategy to restore dysbiosis and intestinal homeostasis and thereby prevent progression towards end stage complicated liver disease.

In this thesis, we showed that the intestine, i.e. its epithelial barrier and microbiota composition, is involved in compensated liver cirrhosis. It would be relevant to further 
investigate whether the observed cirrhosis-related alterations in the intestine can be restored, for example by reinforcing the epithelial barrier and/or modulating the microbiota composition, and thereby prevent progression towards end stage complicated liver disease. This may decrease the high disease burden and health care costs of cirrhotic patients. Furthermore, we showed that non-invasive analysis of exhaled VOCs can predict the presence of compensated cirrhosis among a heterogeneous group of patients with chronic liver diseases, which is of relevance for both patients and society as early diagnosis of cirrhosis can prevent development of severe complications, and thereby improve health-related quality of life and decrease health care costs. In addition, analysis of exhaled VOCs may aid in reducing the number of invasive liver biopsies and could be implemented in daily clinical practice to monitor patients over time. 


\section{References}

1. Anthony PP, Ishak KG, Nayak NC, Poulsen HE, Scheuer PJ, Sobin LH. The morphology of cirrhosis: definition, nomenclature, and classification. Bull World Health Organ 1977;55:521-40.

2. Hoyert DL, Xu J. Deaths: Preliminary Data for 2011. National vital statistics reports; vol 61 no 6. Hyattsville, MD: National Center for Health Statistics. 2011. Available from: http://www.cdc.gov/nchs/data/nvsr/nvsr61/nvsr61_06.pdf.

3. Blachier M, Leleu H, Peck-Radosavljevic M, Valla DC, Roudot-Thoraval F. The burden of liver disease in Europe: a review of available epidemiological data. J Hepatol 2013;58:593-608.

4. Rockey DC, Caldwell SH, Goodman ZD, Nelson RC, Smith AD. Liver biopsy. Hepatology 2009;49: 1017-44.

5. Khaleghi S, Ju JM, Lamba A, Murray JA. The potential utility of tight junction regulation in celiac disease: focus on larazotide acetate. Therap Adv Gastroenterol 2016;9:37-49.

6. Dhiman RK, Rana B, Agrawal S, et al. Probiotic VSL\#3 reduces liver disease severity and hospitalization in patients with cirrhosis: a randomized, controlled trial. Gastroenterology 2014;147:1327-37 e3. 
Dankwoord 


\section{Dankwoord}

Prof. dr. Masclee, beste $A d$, bedankt voor de begeleiding gedurende het gehele promotietraject en de steun en het begrip voor mijn keuze om geen Maag-, Darm- en Leverarts te worden.

Dr. Koek, beste Ger, uw motiverende enthousiasme en uw bewonderenswaardige klinische blik hebben ertoe geleid dat ik koos voor het vak en bovendien het onderzoek. Bedankt voor de mogelijkheid en het vertrouwen. Ik ben blij dat ik nu eindelijk volmondig 'JA' kan zeggen op uw wekelijkse vraag of het boekje al af is!

Dr. Jonkers, lieve Daisy, ontzettend bedankt voor de begeleiding en alle tijd die je daarvoor nam. Dankzij jouw kritisch commentaar heb ik heel veel van je geleerd en heb je de manuscripten naar een hoger niveau gebracht. Dank voor alle steun en de relativerende woorden; "het is zoals het is"!

Daarnaast wil ik ook alle coauteurs bedanken voor jullie inzet en de prettige samenwerking.

In het bijzonder wil ik Prof. dr. Bast, dr. Haenen en hun collega's van de vakgroep Toxicologie bedanken voor de wetenschappelijke input en de leerzame besprekingen.

Prof. dr. van Schooten en dr. Dallinga, hartelijk dank voor de mogelijkheid om te participeren in het VOC onderzoek. Dr. Smolinska, beste Agnieszka, jouw statische analyses en tomeloze inspanningen hebben geleid tot een prachtige publicatie!

Dr. Penders en Danyta, dank voor het analyseren en de hulp bij de interpretatie van de microbiota data. Laten we hopen dat het manuscript snel geaccepteerd wordt!

Beste leden van de beoordelingscommissie, hartelijk dank voor het kritisch doorlezen en beoordelen van het proefschrift.

Alle stafleden, arts-assistenten en medewerkers van de afdeling Maag-, Darm- en Leverziekten van het MUMC; bedankt voor jullie bijdrage bij de inclusie van patiënten en het aanleveren van kostbaar patiëntmateriaal. In het bijzonder dank aan alle proefpersonen die hebben deelgenomen aan de onderzoeken in dit proefschrift; zonder jullie was dit alles niet mogelijk geweest. Tiny, bedankt voor de hulp bij de layout van dit proefschrift.

Natuurlijk wil ik ook de fantastische groep collega PhD's bedanken voor de fijne tijd die ik heb gehad op en naast het werk. Eveline, Elhaseen, Samefko, Paul, Renske, Sander, Mark, Chantal, Bouke, Kirsten, Corinne, Bas, Steven, Fedde, Zlatan, Ellen, Fabienne en Annick, bedankt voor de gezellige lunches, borrels en interesse in mijn onderzoek. Daniël, hoe druk je het ook had, altijd kon ik gevraagd (en ongevraagd) rekenen op je hulp, dankjewel. Mariëlle, bedankt voor je luisterend oor, je hulp bij van alles en nog wat en het steuntje in de rug wanneer dit nodig was. Tim, eerste kamergenoot; ondanks een valse start en mijn hoge eisen aan onze werkomgeving;), hebben we veel 
gelachen. Na enig uitstel, moet jij er dan toch ook aan geloven; succes met promoveren! Esther, Montserrat en Harm-Jan, bedankt voor jullie hulp in het lab. En tot slot dank aan alle studenten die een bijdrage hebben geleverd aan mijn onderzoek.

Lieve vriendinnen en vrienden in Maastricht, 'lichting 2003', 'het vertikaal', 'de vrienden van vroeger' en de dames van Ladies Circle'36 Maasland, dank jullie voor de welkome afleiding tijdens het promotietraject. Lieve Michelle, al meer dan 25 jaar vriendinnen; bedankt dat je er altijd voor me bent. Lieve Evelien, dank voor je vrolijke en nuchtere kijk op alles!

Lieve Celien, dankjewel voor je vriendschap, je lieve en altijd bemoedigende woorden, je onvoorspelbaarheid en je humor. Ik ben trots dat jij als paranimf bij dit belangrijke moment achter me zult staan.

Lieve Ellen, mijn grote zus, ondanks je drukke leven heb je altijd tijd om mij advies te geven. Ik bewonder je onuitputtelijke energie en ambitie, en ben trots op jou en jouw gezinnetje! Wat bof ik met jou als zus en nu als paranimf!

Lieve papa en mama, jullie onvoorwaardelijke liefde en steun is zo vanzelfsprekend dat ik jullie haast zou vergeten. Bedankt voor ALLES! Ik houd ontzettend veel van jullie!

Lieve schoonfamilie, Frank en Coby, bedankt voor de afleiding, interesse en de heerlijke vakanties!

Lieve, lieve Twan, jouw positieve kijk op alles maakt het leven met jou heerlijk! Samen met jou geniet ik van onze twee mannetjes voor wie je een fantastische vader bent. Bedankt voor de motiverende woorden, afleiding en ondersteuning; bedankt voor ALLES! Je bent het beste en mooiste wat me ooit is overkomen! 
Curriculum vitae 


\section{Curriculum vitae}

Kirsten Evelyne Pijls was born on April 2nd 1985 in Heerlen, the Netherlands. After obtaining her gymnasium degree at the Bernardinuscollege in Heerlen in 2003, she started her medical studies at Maastricht University. During this period, she accomplished clinical and scientific traineeships at the Division of Gastroenterology and Hepatology, Maastricht University Medical Center, under the supervision of Dr. G.H. Koek. After graduating in 2009, she received the Kootstra Talent Fellowship. With this, the work presented in this thesis was initiated. From August 2009 to November 2013, Kirsten worked as a PhD student affiliated to the division of Gastroenterology and Hepatology, Maastricht University Medical Center and NUTRIM, the School of Nutrition and Translational Research in Metabolism, Maastricht University, under the supervision of prof. dr. A.A.M Masclee, dr. D.M.A.E. Jonkers and dr. G.H. Koek. In December 2013, she started her residency in Gastroenterology and Hepatology at the department of Internal Medicine, Atrium Medical Center, Heerlen, the Netherlands. In 2015, she decided to stop the residency. In 2016, she finished her thesis and started to work as a physician in youth health care. 
$\overline{182}$ 
List of publications 


\section{List of publications}

Pijls KE, Tedjo D, Koek GH, Budding AE, Savelkoul PH, Masclee AA, Penders J, Jonkers DM. Altered fecal and duodenal microbial profiles are associated with disease severity in liver cirrhosis patients. Submitted.

Pijls KE, Jonkers DM, Elizalde M, Drittij-Reijnders MJ, Haenen GR, Bast A, Masclee AA, Koek $\mathrm{GH}$. Is intestinal oxidative stress involved in patients with compensated liver cirrhosis? Ann Hepatol. 2016; 15:402-409

Pijls KE, Smolinska A, Jonkers DM, Dallinga JW, Masclee AA, Koek GH, van Schooten FJ. A profile of volatile organic compounds in exhaled air as a potential non-invasive biomarker for liver cirrhosis. Sci Rep. 2016

Pijls KE, de Vries H, Nikkessen S, Bast A, Wodzig WK, Koek GH. Critical appraisal of ${ }^{13} \mathrm{C}$ breath tests for microsomal liver function: aminopyrine revisited. Liver Int. 2014; 34:487-94

Pijls KE, Koek GH, Elamin EE, de Vries H, Masclee AA, Jonkers DM. Large intestine permeability is increased in patients with compensated liver cirrhosis. Am J Physiol Gastrointest Liver Physiol. 2014; 306: G147-53

Pijls KE, Jonkers DM, Elamin EE, Masclee AA, Koek GH. Intestinal epithelial barrier function in liver cirrhosis: an extensive review of the literature. Liver Int. 2013; 33:1457-69 
